\title{
RNA methylation in
}

\section{Cardiac Hypertrophy and Heart Failure}

\author{
Dissertation
}

\author{
For the award of the degree \\ "Doctor rerum naturalium" \\ of the Georg-August-Universität Göttingen
}

within the doctoral program Molecular Medicine

at the Georg-August University School of Science (GAUSS)

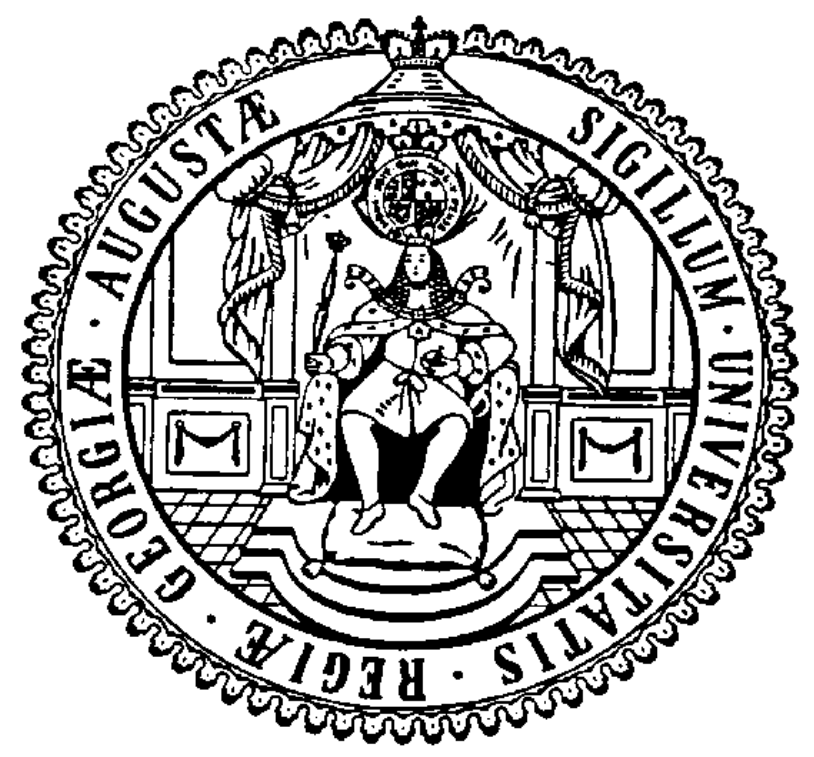

submitted by

\section{Eric Buchholz}

born in Bergisch Gladbach, Germany

Göttingen, August 2020 



\section{Thesis Committee/Examination Board}

Prof. Dr. André Fischer (first reviewer)

Epigenetics and Systems medicine in Neurodegenerative Diseases

German Center for Neurodegenerative Diseases, Göttingen, Germany

Prof. Dr. Karl Toischer (second reviewer)

Cardiac Remodeling

Clinic for Cardiology and Pneumology, University Medical Center Göttingen, Germany

\section{Prof. Dr. Steven Johnsen}

Gene Regulatory Mechanisms and Molecular Epigenetics

Division of Gastroenterology and Hepatology, Mayo Clinic, Rochester, MN, USA

\section{Further members of the Examination Board}

Prof. Dr. Bernd Wollnik

Human Genetics, University Medical Center Göttingen, Germany

Prof. Dr. Wolfram-Hubertus Zimmermann

Clinic for Pharmacology and Toxicology, University Medical Center Göttingen, Germany

\section{Prof. Dr. Dörthe Katschinski}

Institute of Cardiovascular Physiology, University Medical Center Göttingen, Germany

Date of oral examination: 


\section{Affidavit}

I hereby declare that I have written the dissertation

'RNA methylation in Cardiac Hypertrophy and Heart Failure”

independently with no other aids or sources than quoted.

I want to mention here, that parts of the results in this thesis were already published, and I will attach the manuscript to this thesis. Some figures were taken from the paper and modified. As the publication is Open Source and I am co-first author, I am allowed to use the data in my thesis since data generation for the publication was a main part of my PhD-Project.

Berulava $T^{*}$, Buchholz $E^{*}$, Elerdashvili $V$, et al. Changes in m6A RNA methylation contribute to heart failure progression by modulating translation. Eur J Heart Fail. 2020;22(1):54-66. doi:10.1002/ejhf.1672

${ }^{*}$ Contributed equally to the manuscript

The Publication is available under the following link:

https://pubmed.ncbi.nlm.nih.gov/31849158/

Eric Buchholz

Göttingen, 24.08.2020 


\section{Table of Content}

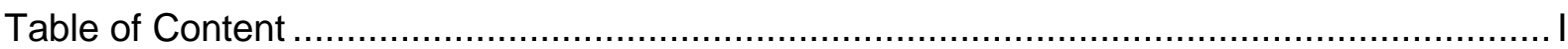

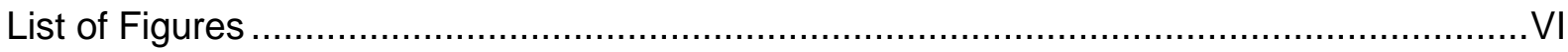

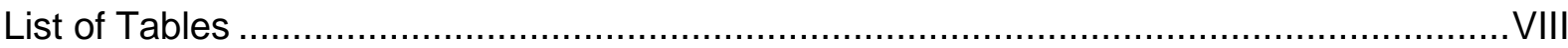

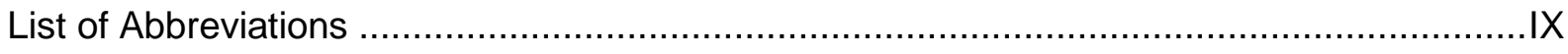

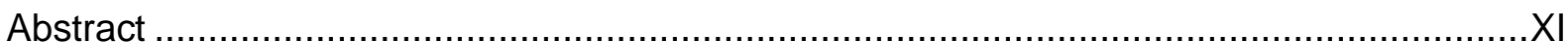

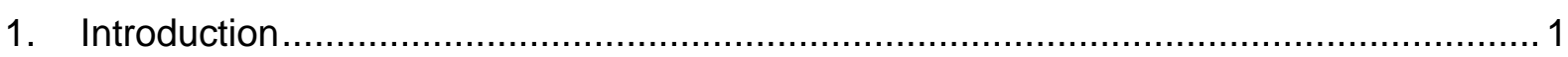

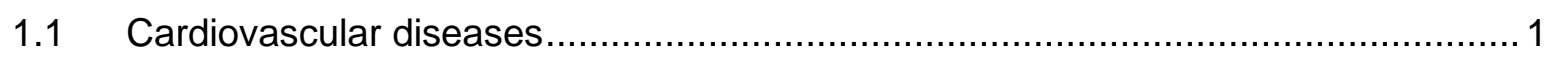

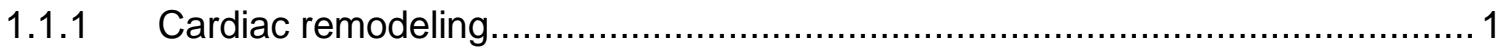

1.1.2 Molecular mechanisms of cardiac remodeling ......................................... 2

1.1.3 Therapeutic options for treatment of heart failure ......................................... 3

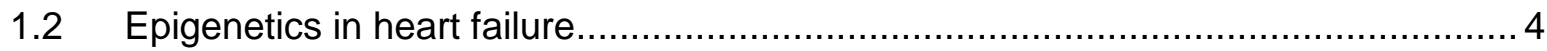

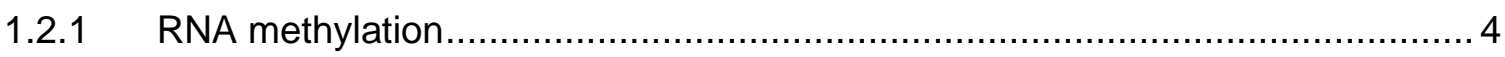

1.2.2 Reading and writing the m6A code - dynamic and reversible ........................5

1.2.3 The various RNA methylation mediated effects - reading and interpreting the

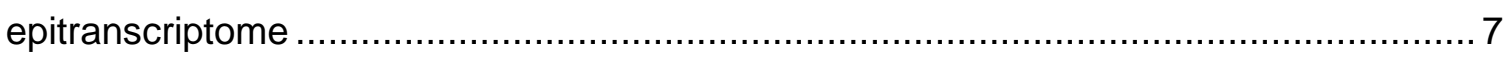

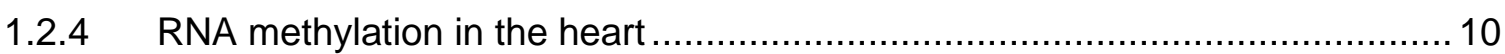

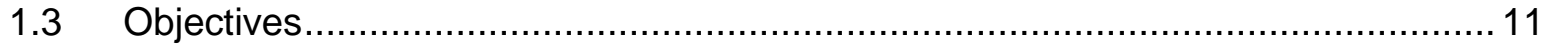

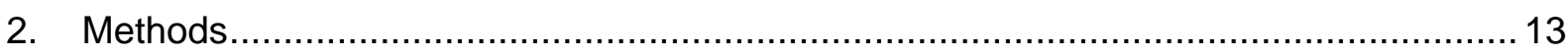

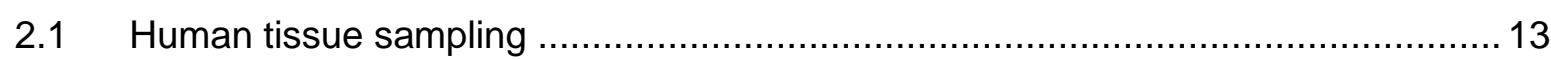

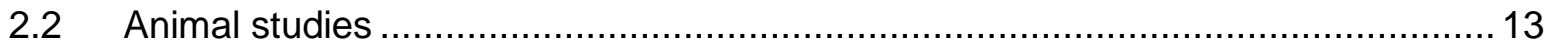

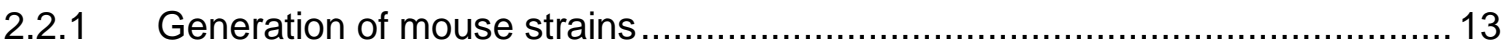

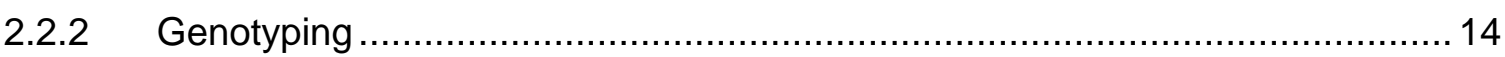

2.2.3 Transverse aortic constriction ............................................................ 16

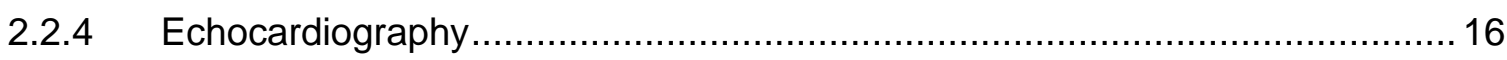

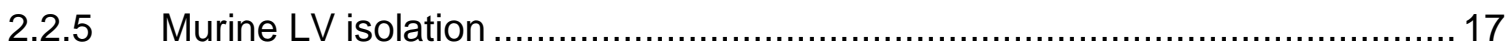

2.2.6 Langendorff perfused heart cardiomyocyte isolation ................................... 18

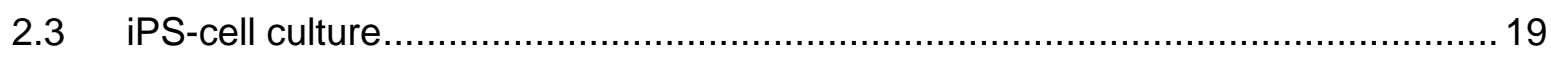

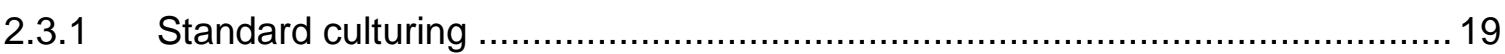

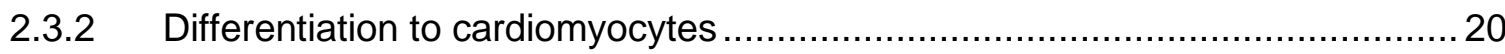

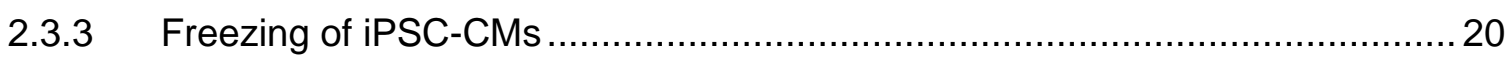




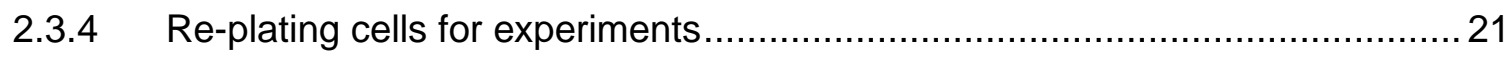

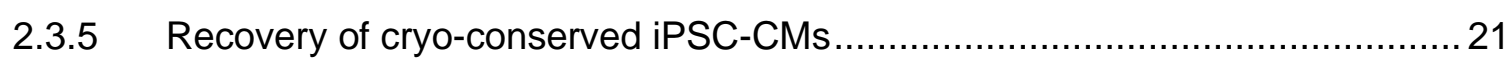

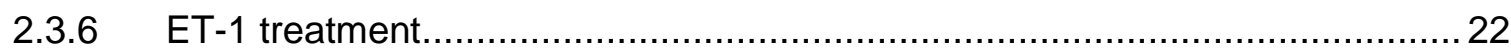

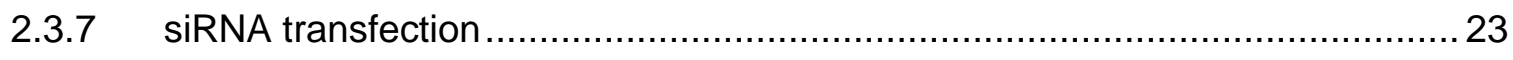

2.3.8 Combination of siRNA transfection and ET-1 treatment............................. 24

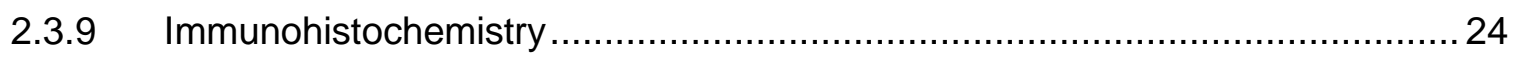

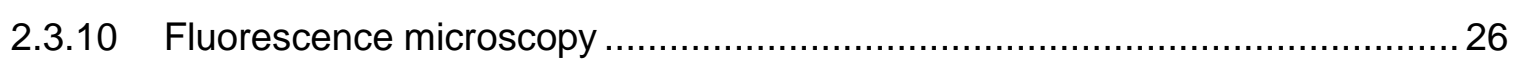

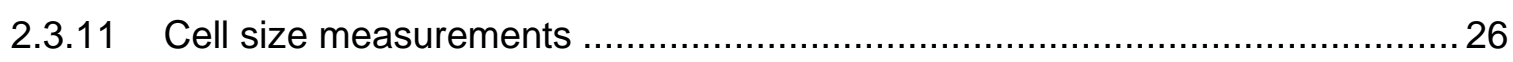

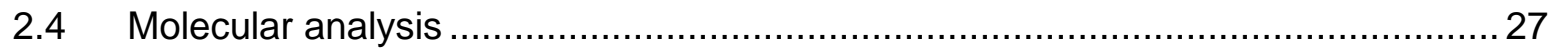

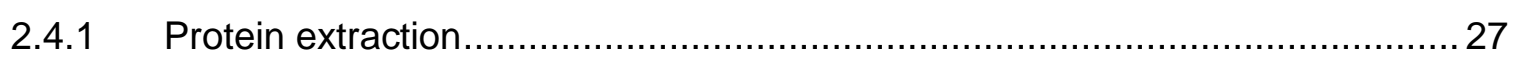

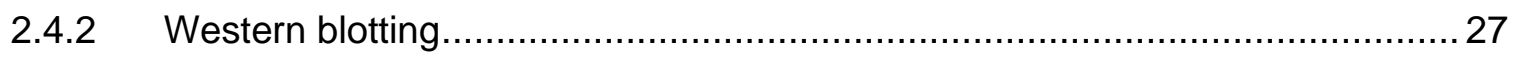

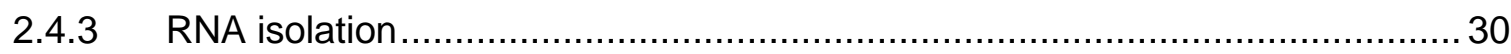

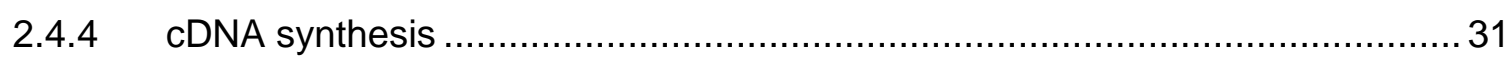

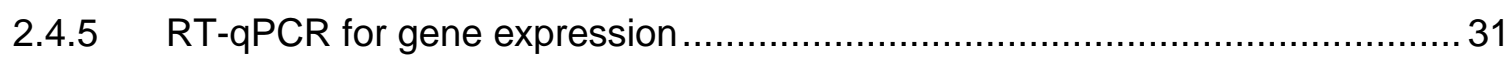

2.4.6 RT-qPCR verification of differentially methylated transcripts........................ 33

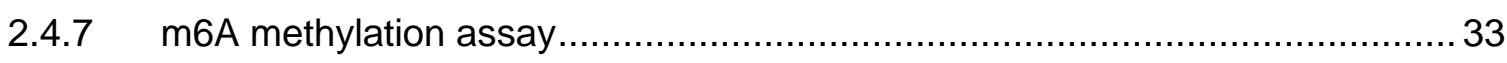

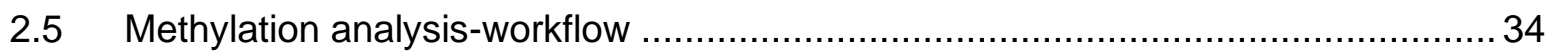

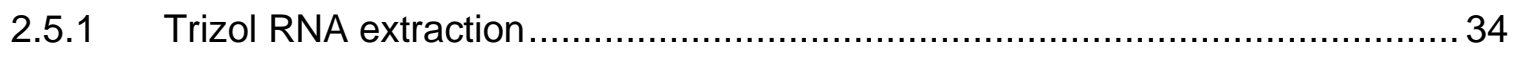

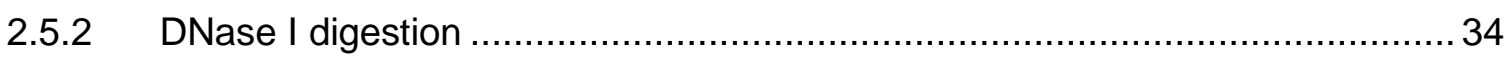

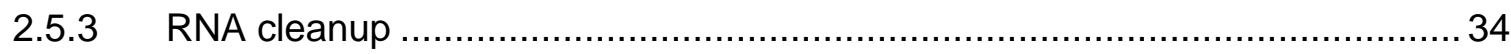

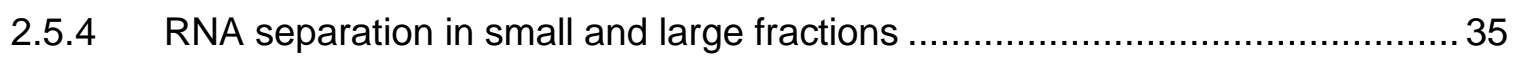

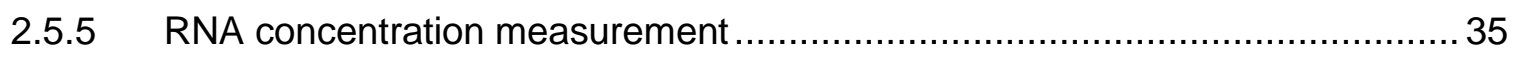

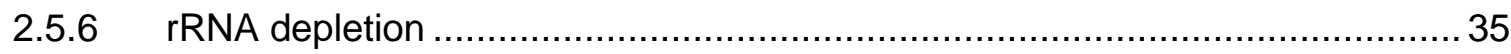

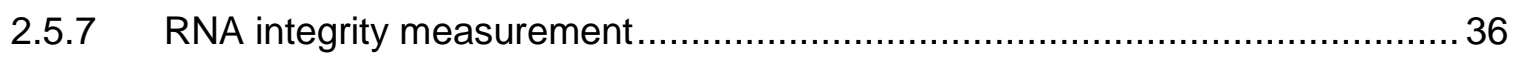

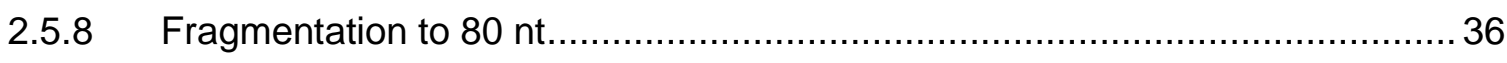

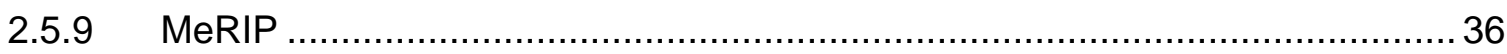

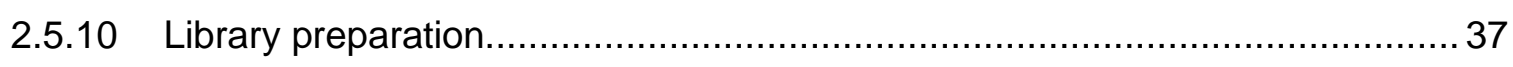

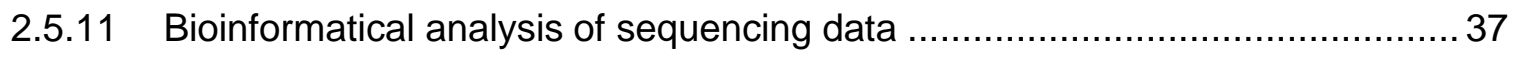

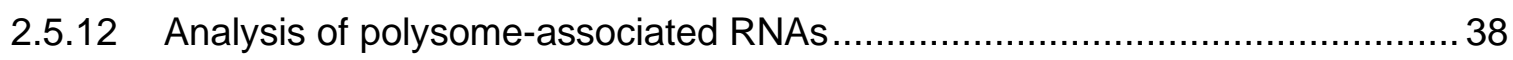

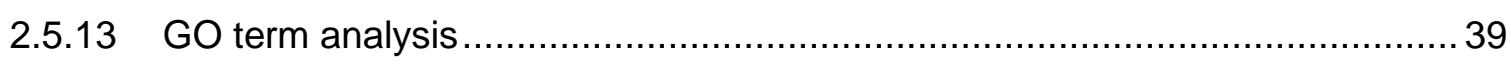


2.6 Software used for visualization and statistical analysis 39

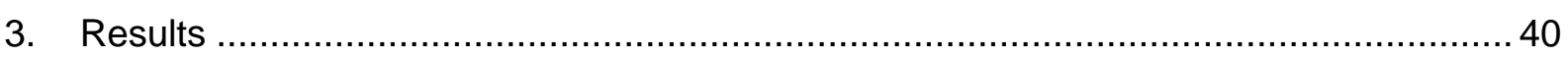

3.1 m6A Methylation in mouse and human hearts ......................................... 40

3.1.1 Basal characterization of the m6A methylation level in hearts .......................... 40

3.1.1.1 The methylation machinery is present in cardiac tissue ........................... 40

3.1.1.2 Methylation characteristics of healthy mouse and human hearts................ 41

3.1.1.3 Comparison of methylation in healthy mouse and human hearts demonstrates

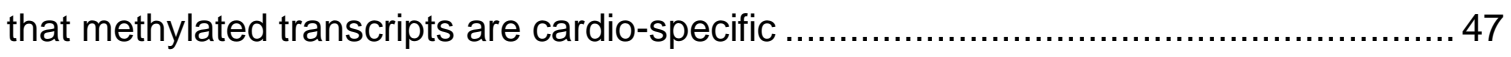

3.1.2 The Transcriptome changes in hypertrophy and heart failure ......................... 48

3.1.2.1 TAC-induced cardiac hypertrophy ................................................. 48

3.1.2.2 Persistent TAC-induced pressure overload progresses to heart failure .......50

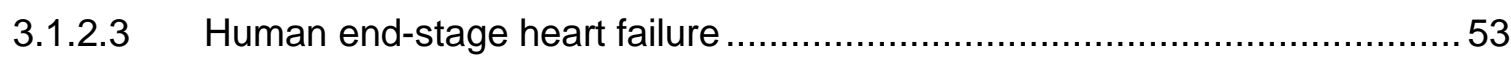

3.1.3 Methylation changes in the progression to heart failure .................................. 54

3.1.3.1 Methylation changes in mouse hypertrophy ...................................... 54

3.1.3.2 Methylation changes in mouse heart failure ..................................... 57

3.1.3.3 Methylation changes in human end-stage heart failure ............................60

3.1.3.4 Comparison of the mouse and human heart failure epitranscriptome ..........63

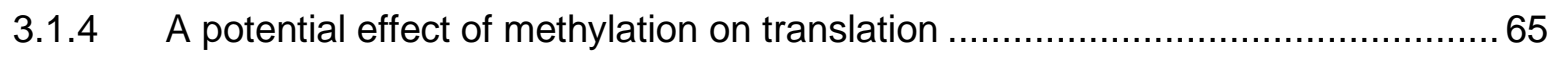

3.1.4.1 Methylation-mediated expression level changes may not be the main

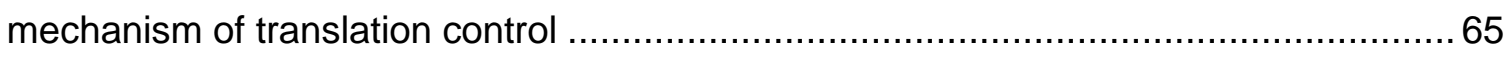

3.1.4.2 The effect of RNA methylation on translation by polysomal analysis .......... 66

3.1.4.3 Correlation of methylation and ribosomal occupancy …............................67

3.1.4.4 A potential transcription-independent translation mechanism .................... 69

3.2 Manipulation of the methylation machinery in iPSC-CMs ................................ 72

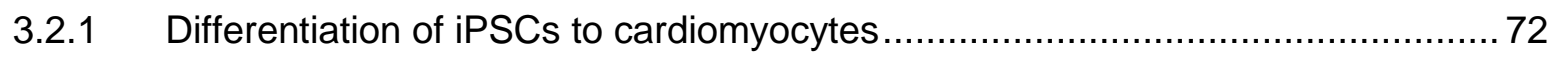

3.2.2 Establishing an ET-1-induced hypertrophy model in iPSC-CMs ..................... 73

3.2.3 Establishing siRNA- mediated silencing of FTO in iPSC-CMs ....................... 75

3.2.4 Establishing a combined siRNA-transfection and ET-1 stimulation protocol ....... 78

3.2.5 The effect of silencing FTO on ET-1 induced hypertrophic growth.................... 82

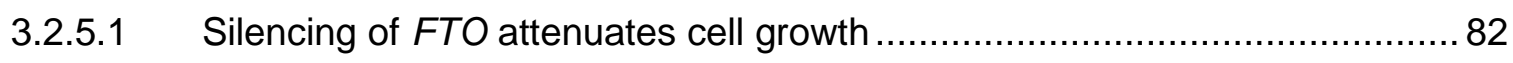


3.2.5.2 ET-1 stimulation does not affect FTO expression per se 84

3.2.5.3 Molecular analysis of attenuated cell growth by ANP level assessment ......84

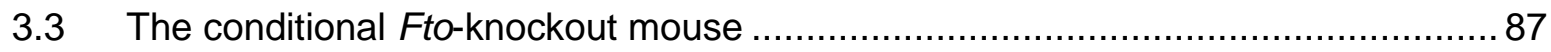

3.3.1 Validation of the cardiomyocyte-specific knockout of Fto................................ 87

3.3.2 Phenotyping the knockout mouse model .............................................. 90

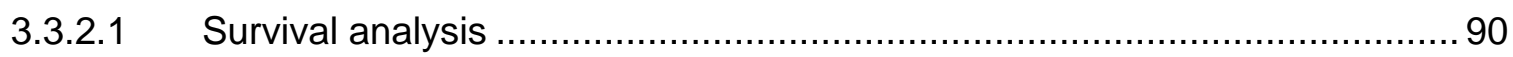

3.3.2.2 Cardiac performance and morphometry of knockout-mice ........................ 91

3.3.3 Response to TAC-induced hypertrophy in the Fto-cKO mouse model...............93

3.3.3.1 TAC surgery with a $27-G$ needle ..................................................... 93

3.3.3.2 TAC surgery with $26 \mathrm{G}$ needle ...................................................... 95

3.3.3.3 Fto ${ }^{\text {cKO }}$ mice show different cardiac remodeling than Cre $^{\text {Control }}$ mice ............... 96

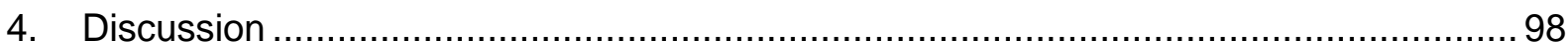

4.1 The RNA methylation machinery allows dynamic methylation in cardiac tissues ...98

4.2 The epitranscriptome of mouse and human hearts elucidated ............................99

4.3 Hypertrophy and heart failure were successfully induced in mice ...................... 100

4.4 Transcriptomic changes in response to cardiac stress account for cardiac plasticity

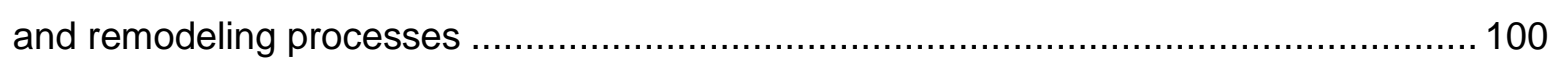

4.5 The epitranscriptome changes in response to cardiac stress ........................... 101

4.5.1 Methylation changes outnumber expression changes by far....................... 101

4.5.2 Only a small number of transcripts is affected at the methylation and expression

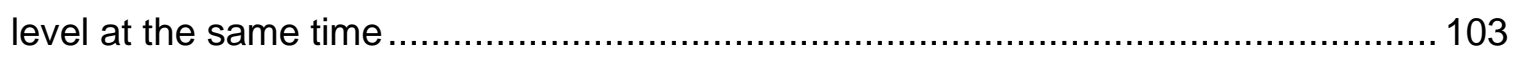

4.5.3 Transcripts changed at their methylation level reveal substantially different Functions than those changed at expression level - a fast response mechanism....... 103

4.5.4 m6A RNA methylation affects translation............................................ 105

4.5.5 A potential mechanism of transcription independent translation control ......... 105

4.5.6 m6A methylation in hypertrophy and heart failure may act in a 'fast-response' mechanism by accelerating translation and turnover ........................................... 106

4.6 FTO affects the hypertrophic response in vitro and in vivo............................111

4.6.1 Silencing of FTO attenuates hypertrophy in vitro ................................... 111

4.6.2 A cardiomyocyte-specific Fto knockout in vivo results in a mild cardiac phenotype 112 
4.6.3 The cardiomyocyte-specific depletion of FTO in vivo leads to a worsened

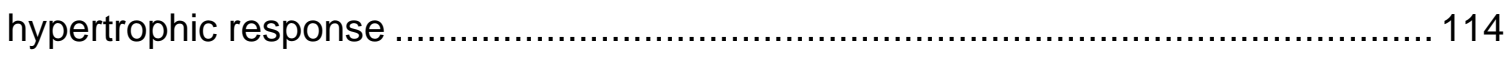

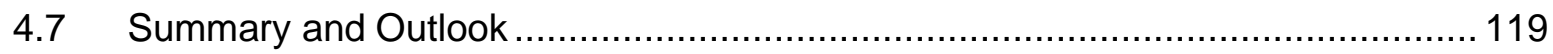

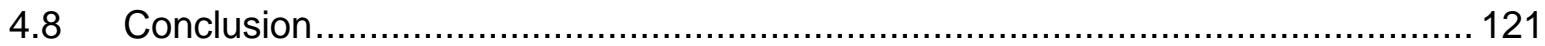

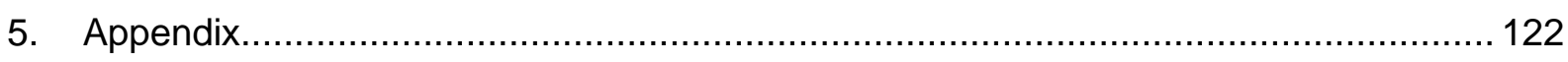

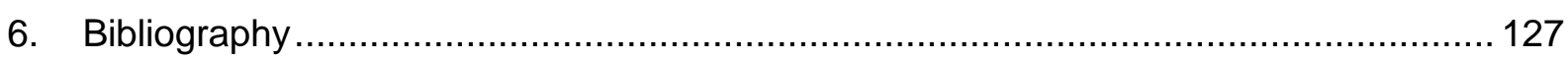

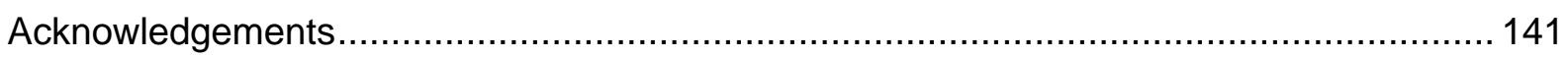

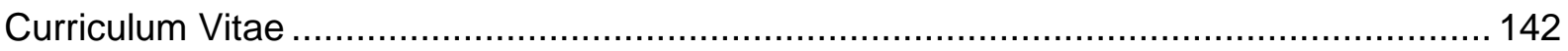




\section{List of Figures}

Figure 1.1 Cardiac hypertrophy and progression to heart failure- schematic overview ..... 2

Figure 1.2 Dynamic and reversible m6A RNA methylation.

Figure 3.1 The m6A methylation machinery is present in mouse and human heart tissue .. 41

Figure 3.2 Typical methylation patterns were detected in mouse and human heart tissue ... 43

Figure 3.3 Many transcripts carry methylation marks in several regions.

Figure 3.4 Methylation marks in different regions are associated with different biological functions and reveal a correlation with expression level.

Figure 3.5 Transcripts methylated in both mouse and human tissues play a role in cardiospecific pathways

Figure 3.6 Cardiac hypertrophy is observed one week after TAC with a change in gene expression

Figure 3.7 Mice develop heart failure with gene expression changes 8-weeks post-TAC.....51 Figure 3.8 Select genes are continuously deregulated during the progression from hypertrophy to heart failure and are associated with pathways of cardiac plasticity 52

Figure 3.9 Gene expression changes in human end-stage heart failure. 53

Figure $3.10 \mathrm{~m} 6 \mathrm{~A}$ methylation changes outnumber expression changes in mouse hypertrophy

Figure 3.11 Differentially methylated transcripts in hypertrophy are associated with catabolic and metabolic processes as well as signal transduction pathways

Figure $3.12 \mathrm{~m} 6 \mathrm{~A}$ methylation changes outnumber expression changes in mouse heart failure

Figure 3.13 Differentially methylated transcripts in heart failure are associated with catabolic and metabolic processes as well as signal transduction pathways.

Figure 3.14 Methylation changes outnumber expression changes upon heart failure in human tissue.

Figure 3.15 Differentially methylated transcripts in human heart failure are implicated in signal transduction pathways

Figure 3.16 Many differentially methylated transcripts in heart failure tissue of mouse and human are of cardiac specificity.

Figure 3.17 Transcripts changed at the methylation level are most likely different from transcripts changed at the expression level

Figure 3.18 The polysomal occupancy of differentially methylated mRNAs is altered upon TACinduced heart failure in mice

Figure 3.19 RNA methylation correlates with polysomal occupancy and therefore translation in heart failure tissue of mice 
Figure 3.21 CALM1 protein level is decreased despite unaltered expression level in mouse

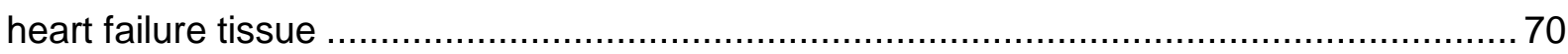

Figure 3.22 CALM1 is hypomethylated and its protein level decreases in human heart failure tissue, despite being unaltered at the expression level. .............................................. 71

Figure 3.23 Concentration determination of ET-1 treatment.......................................... 74

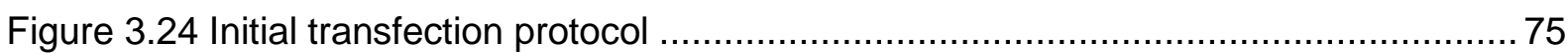

Figure 3.25 A stable FTO knockdown on the mRNA level was induced 48 hours after

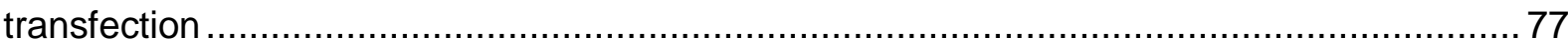

Figure 3.26 Combined siRNA-transfection and ET-1 treatment protocol ............................ 78

Figure 3.27 The Fto-KD is still active 48 hours after siRNA removal ................................ 79

Figure 3.28 Adapted siRNA transfection protocol combined with ET-1 stimulation................80

Figure 3.29 The FTO protein level is significantly decreased following the adapted protocol 81

Figure 3.30 FTO-KD attenuates cell growth .............................................................. 83

Figure 3.31 The FTO protein level is unchanged upon ET-1 treatment ............................. 84

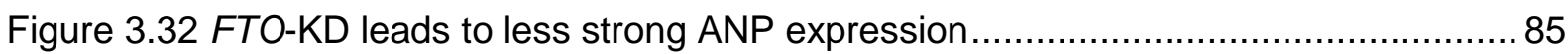

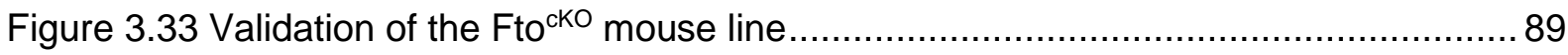

Figure 3.34 Basal survival is not affected by the Fto-KO until 8-months of age ...................91

Figure 3.35 Basal echocardiographic analysis of Fto ${ }^{\mathrm{KKO}}$ animals suggests a dilatative basal

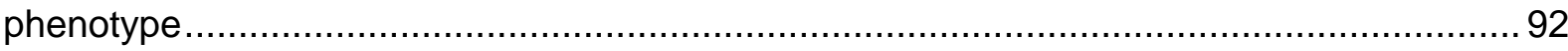

Figure 3.36 Echocardiographic characterization of Fto ${ }^{\mathrm{ckO}}$ animals one week after TAC with 27G needle

Figure 3.37 Echocardiographic characterization of Fto ${ }^{\mathrm{cKO}}$ animals 4 weeks after TAC surgery with a $27-G$ needle.

Figure 3.38 Echocardiographic characterization of $\mathrm{Fto}^{\mathrm{ckO}}$ animals one week after TAC with a 26-G needle 96

Figure 3.39 Fto-KO affects the hypertrophic response 97

Figure 4.1 Transcripts changed at the methylation level are substantially different from those changed at the expression level - a potential fast-translation with fast-turnover mechanism

Figure 4.2 FTO depletion leads to maladaptation and early dilatation, potentially by attenuating hypertrophic growth 116

Figure 5.1 Full blots of the methylation machinery in mouse and human LV tissue 123

Figure 5.2 Fill blots Calmodulin1 Mouse and Human 124

Figure 5.3 Testing different siRNAs targeting FTO-mRNA for effectivity 125

Figure 5.4 Full blot ANP 126

Figure $5.5 \mathrm{~m} 6 \mathrm{~A}$ Assay did not reveal changed m6A levels in Fto ${ }^{\text {cKO }}$ mice compared to Cre Control $^{\text {. }}$ 


\section{List of Tables}

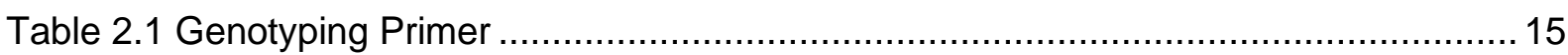

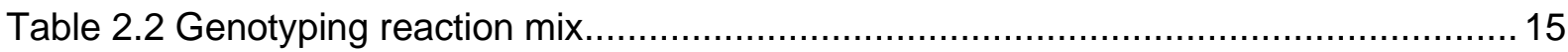

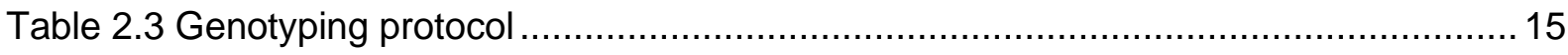

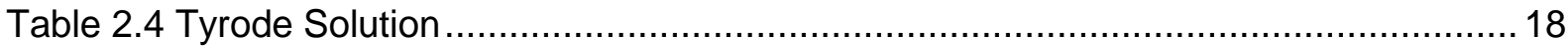

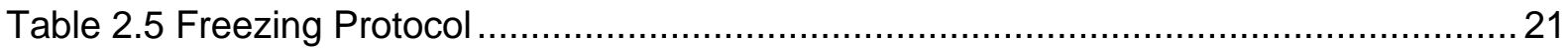

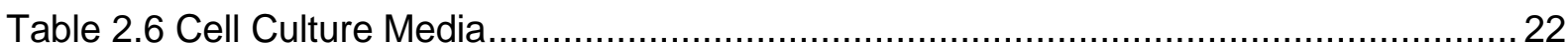

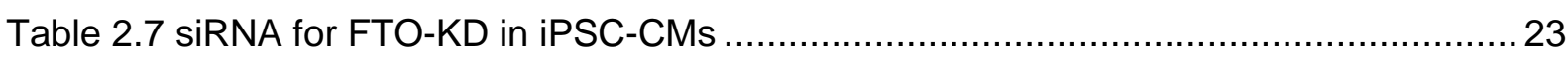

Table 2.8 Lipoefectamine Transfection Mix .................................................................. 23

Table 2.9 HiPerFect $\AA$ Transfection Mix .................................................................... 24

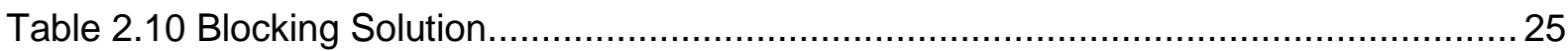

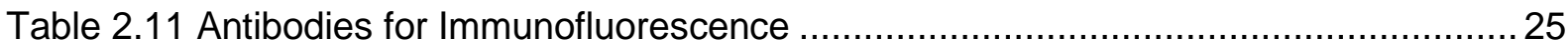

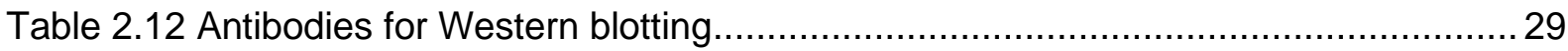

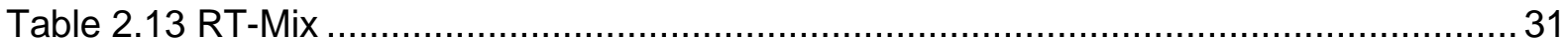

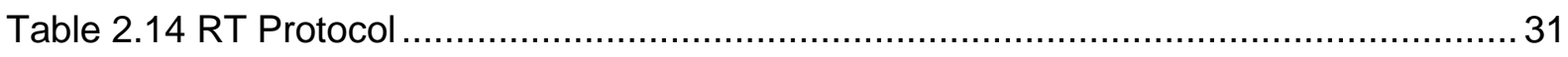

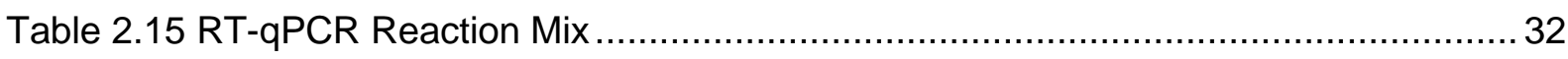

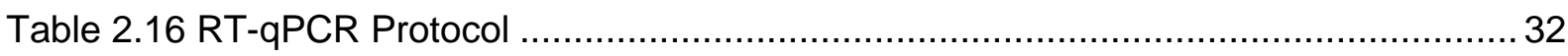

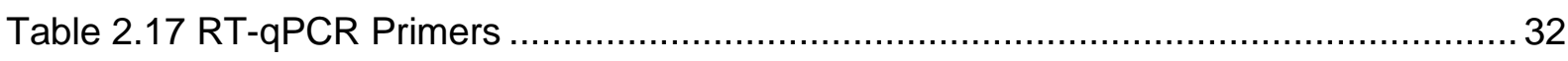

Table 2.18 Universal Probe Library Primer and Probes .................................................. 33

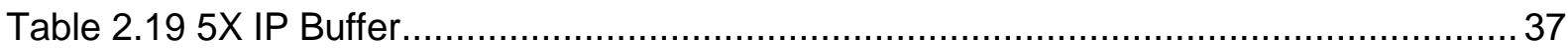

Table 5.1 Patient Data of healthy, non-failing tissue samples ....................................... 122

Table 5.2 Patient Data of end-stage HF tissue samples.................................................. 122 


\section{List of Abbreviations}

\begin{tabular}{|c|c|c|c|}
\hline $26 G / 27 G$ & 26 Gauge/ 27 Gauge & MAPK & Mitogen-activated protein kinase \\
\hline 3' UTR & 3' untranslated region & MDM2 & mouse double minute 2 homolog \\
\hline 5' UTR & 5' untranslated region & MEF2 & $\begin{array}{l}\text { myocyte enhancer factor-2 } \\
\text { fusion protein of cre- } \\
\text { recombinase and modified }\end{array}$ \\
\hline ACE & angiotensin-converting-enzyme & MerCreMer & $\begin{array}{l}\text { estrogen receptor } \\
\text { Methylated RNA }\end{array}$ \\
\hline ACTA1 & actin alpha 1 & MeRIP-seq & immunoprecipitation Sequencing \\
\hline ALKBH5 & AlkB Homolog 5 & METTL14 & Methyltransferase Like 14 \\
\hline Alpha-MHC & Myosin heavy chain Alpha & METTL16 & Methyltransferase Like 16 \\
\hline ANP & Atrial natriuretic peptide & METTL3 & Methyltransferase Like 3 \\
\hline AT & Angiotensin & METTL5 & Methyltransferase Like 5 \\
\hline$A U$ & arbitrary units & miRNA & micro RNA \\
\hline AwTh & Anterior wall thickness & mRNA & messenger RNA \\
\hline BNP & Brain natriuretic peptide & Myh6 & Myosin heavy chain 6 \\
\hline CALM1 & $\begin{array}{l}\text { Calmodulin } 1 \\
\text { calcium/calmodulin-dependent }\end{array}$ & Myh7 & Myosin heavy chain 7 \\
\hline CaMKII & protein kinase type II & ncRNA & non-coding RNA \\
\hline cDNA & complementary DNA & NF & non-failing \\
\hline CDS & coding sequence & NFAT & nuclear factor of activated T-cells \\
\hline cKO & cardiomyocyte specific knockout & NGS & Next Generation Sequencing \\
\hline CMs & cardiomyocytes & Nppa & gene encoding ANP \\
\hline CVD & Cardiovascular diseases & Nppb & gene encoding BNP \\
\hline DAPI & 4',6-diamidino-2-phenylindole & nrc & normalized read count \\
\hline DC1 & YTH domain containing protein 1 & NRCM & neonatal rat cardiomyocytes \\
\hline DC2 & YTH domain containing protein 2 & $\mathrm{OE}$ & overexpression \\
\hline DCM & $\begin{array}{l}\text { Dilated cardiomyopathie } \\
\text { YTH domain containing family }\end{array}$ & ON & overnight \\
\hline DF1-3 & protein 1-3 & PCR & polymerase chain reaction \\
\hline DICER1 & an endoribonuclease & pCTRL & pCTRL1.1 cell line \\
\hline DMSO & $\begin{array}{l}\text { Dimethylsulfoxid } \\
\text { Dulbecco's Phosphate buffered }\end{array}$ & PE & Phenylephrine \\
\hline DPBS & Saline & PKA & protein kinase $\mathrm{A}$ \\
\hline DTT & Dithiothreitol & $\mathrm{PKC} \alpha$ & protein kinase $\mathrm{C} \alpha$ \\
\hline EF & $\begin{array}{l}\text { ejection fraction } \\
\text { eukaryotic translation initiation }\end{array}$ & PKG & protein kinase G \\
\hline elf3 & $\begin{array}{l}\text { factor } 3 \\
\text { eukaryotic translation initiation }\end{array}$ & PLN & Phospholamban \\
\hline elf4E & $\begin{array}{l}\text { factor } 4 \mathrm{E} \\
\text { eukaryotic translation initiation }\end{array}$ & PO & pressure overload \\
\hline Elf5A & $\begin{array}{l}\text { factor } 5 \mathrm{~A} \\
\text { extracellular signal-regulated }\end{array}$ & POI & protein of interest \\
\hline ERK & kinase & PwTh & Posterior wall thickness \\
\hline ET-1 & Endothelin-1 & RAAS & renin angiotensin system \\
\hline exRNA & extracellular RNA & RBM20 & RNA binding morif protein 20 \\
\hline FBS & fetal bovine serum & RBP & RNA binding proteins \\
\hline FDR & false detection rate & RISC & RNA induced silencing complex \\
\hline
\end{tabular}




\begin{tabular}{|c|c|c|c|}
\hline Flpo & Flipe site specific recombinase & RNAi & RNA interference \\
\hline FOX01 & forkhead box protein 1 & ROS & reactive oxygen species \\
\hline FOXO4 & $\begin{array}{l}\text { forkhead box protein } 4 \\
\text { fat mass and obesity associated } \\
\text { gene (FTO alpha-ketoglutarate }\end{array}$ & rRNA & ribosomal RNA \\
\hline FTO & $\begin{array}{l}\text { dependent dioxygenase) } \\
\text { Glyceraldehyde 3-phosphate }\end{array}$ & RT & room temperature \\
\hline GAPDH & dehydrogenase & RT-qPCR & quantitative real-time PCR \\
\hline GATA4 & GATA Binding Protein 5 & RWT & relative wall thickness \\
\hline GO & gene ontology & RYR2 & $\begin{array}{l}\text { ryanodine receptor } 2 \\
\text { gene encoding } 40 S \text { ribosomal }\end{array}$ \\
\hline HF & $\begin{array}{l}\text { heart failure } \\
\text { human induced pluripotent stem }\end{array}$ & s18 & protein S18 \\
\hline iPSC & $\begin{array}{l}\text { cells } \\
\text { human induced pluripotent stem }\end{array}$ & SAM & $\begin{array}{l}\text { S-adenosyl methionine } \\
\text { Src-Associated substrate in }\end{array}$ \\
\hline iPSC-CM & $\begin{array}{l}\text { cell derived cardiomyocytes } \\
\text { Heterogenous Nuclear }\end{array}$ & SAM68 & Mitosis of 68 kDa \\
\hline HNRNP & Ribonucleoprotein & SC35 & Splicing factor SC35 \\
\hline HRP & horseradish peroxidase & SD & standard deviation \\
\hline HT & hypertrophy & SEM & $\begin{array}{l}\text { standard error of the mean } \\
\text { sarcoplasmic/endoplasmic }\end{array}$ \\
\hline ICM & Ischemic cardiomyopathy & SERCA & reticulum calcium ATPase \\
\hline IF & innmunofluorescence & Sham & placebo surgery for TAC control \\
\hline Input & total RNA fraction for NGS & siRNA & $\begin{array}{l}\text { small interfering RNA } \\
\text { SET and MYND Domain }\end{array}$ \\
\hline IP & immunoprecipitation & SMYD1 & Containing 1 \\
\hline Iped & immunoprecipitated samples & SnRNA & $\begin{array}{l}\text { small nuclear RNA } \\
\text { Serine/Arginine-rich splicing }\end{array}$ \\
\hline KD & knockdown & SRSF1 & $\begin{array}{l}\text { factor } 1 \\
\text { beta-adrenergic receptor }\end{array}$ \\
\hline ко & knockout & ß-blockers & blockers \\
\hline LCR & Low-complexity region & TAC & $\begin{array}{l}\text { transverse aortic constriction } \\
\text { tris buffered saline with Tween- }\end{array}$ \\
\hline LIN28A & Lin-28 Homolog A & TBS-T & 20 \\
\hline LLPS & Liquid-liquid phase separation & TL & $\begin{array}{l}\text { tibia length } \\
\text { TRBA Methyltransferase Subunit }\end{array}$ \\
\hline IncRNA & long non-coding RNA & TRMT112 & $11-2$ \\
\hline loxP & floxed sequence site & tRNA & transfer RNA \\
\hline LV & $\begin{array}{l}\text { left ventricle } \\
\text { left ventricular internal }\end{array}$ & TZV & Thiazovivin \\
\hline LVID & dimension & WNT & a proto-oncogene \\
\hline $\mathrm{m} 2 \mathrm{~A}$ & 2-methyladenosine & WT & wildtype \\
\hline m5C & 5-methylcytosine & WTAP & Wilms tumor associated protein \\
\hline $\mathrm{m} 6 \mathrm{~A}$ & N6-Methyladenosine & WTD2 & $\begin{array}{l}\text { WT-D2-1U cell line } \\
\text { YTH domain containing family }\end{array}$ \\
\hline m6Am & N6-,2'-O-dimethyladenosine & YTH & $\begin{array}{l}\text { protein } \\
\text { Zinc Finger CCHC-Type }\end{array}$ \\
\hline & & ZCCHC4 & Containing 4 \\
\hline
\end{tabular}




\section{Abstract}

Cardiovascular diseases (CVDs) are the leading cause of hospital admission and mortality worldwide. Heart failure (HF) is one of the most common CVDs and is characterized by a reduced cardiac function and left ventricular dilatation resulting in the inability of the heart to supply sufficient blood-flow. Upon cardiac stress, for example by heart valve defects or pressure overload (PO) from aortic stenosis, growth of cardiomyocytes (CMs) occurs. This initial hypertrophic $(\mathrm{HT})$ adaptation preserves cardiac performance. Persistent hemodynamic stress leads to decompensation of the heart resulting in HF. Progression to HF is accompanied by underlying molecular, cellular, and interstitial changes, termed cardiac remodeling. Treatment options concentrate on inhibition of neuroendocrine stimulation or deregulated signaling pathways, but this has only limited efficacy and the morbidity and mortality of HF patients remains high. Therefore, other therapeutic options need to be investigated. Aberrant gene expression, such as re-expression of the fetal gene program, is a key-event in HF progression. Recent research suggested that changes in gene expression are not only regulated by transcription factors, but also by epigenetic processes such as non-coding RNAs and histone or DNA modifications. For example, DNA methylation and histone modifications such as acetylation or methylation can influence transcription of cardio-specific gene programs. Another less studied epigenetic modification is RNA methylation. Similar to DNA, RNA can be post-transcriptionally modified. The most prevalent modification is methylation of the adenosine base, termed $\mathrm{m} 6 \mathrm{~A}$ methylation. This $\mathrm{m} 6 \mathrm{~A}$ methylation is a dynamic and reversible process, mediated by so-called 'writer' and 'eraser' proteins adding or removing methylation marks on RNA. m6A methylation has been found in all classes of RNA, including mRNA, rRNA, tRNA, snRNA, and miRNA. Recent research has suggested that RNA methylation can affect splicing, mRNA transport, translation, storage, or decay. These effects were shown to be either mediated directly by conformational changes of methylated RNA or by so called 'reader' proteins, which recognize methylation marks. This adds an additional layer of transcriptional and translational control.

m6A RNA methylation was first discovered in the 1970s, but long thought to be of minor importance. Newly developed methods to detect and map m6A methylation in 2012 allowed for more thorough investigation of the modification and gave rise to the term 'epitranscriptome', describing the RNA methylation patterns which govern RNA regulation. The epitranscriptome has since been studied intensively in various diseases and fields of biology, including many forms of cancer, neurodegenerative diseases, and developmental biology. However, little is known about the role of m6A methylation in cardiac diseases such as HT and HF. Only recently have a few publications described RNA methylation in ischemic cardiac tissue and hypoxia, or 
the deregulation of $\mathrm{m} 6 \mathrm{~A}$ methylation in response to hypertrophic stimuli in cell culture. Until now, the global epitranscriptome of the heart has never been described.

In my doctoral work, I have analyzed and characterized the global epitranscriptome of healthy human and mouse heart tissue. My analysis demonstrated that a considerable number of detected transcripts carried m6A methylation marks. Furthermore, many of the identified methylated transcripts were found in both mouse and human tissue and were of cardiac specificity, underlining the importance of this RNA modification in cardiac tissues. To better understand the role of these modification in cardiac HT and HF, I analyzed m6A RNA methylation changes in a mouse model of $\mathrm{PO}$ as well as in cardiac tissue from human endstage HF patients. Interestingly, many more transcripts were changed at the methylation level than on the expression level. Furthermore, transcripts altered at the methylation level tend to code for proteins participating in metabolic, catabolic and signal transduction pathways, whereas transcripts with altered expression generally code for completely different pathways, such as those involved in cardiac plasticity and remodeling. Since many transcripts were only altered at the methylation level, polysomal profiling was applied to elucidate if $\mathrm{m} 6 \mathrm{~A}$ methylation impacts translation, which indeed was the case. From this analysis, I propose a new mechanism of transcription independent translation regulation by RNA methylation. I could validate that protein levels change in correlation with altered methylation while the expression level remains unchanged. Furthermore, I hypothesize a mechanism of fast-translation with fast-turnover mediated by m6A methylation which allows CMs to adapt to stressful conditions.

Further, I investigated the effect of manipulation of the RNA demethylase FTO on hypertrophic responses in vitro and in -vivo. For in vitro studies, a human induced pluripotent stem cell (iPSC) model was used. iPSCs were differentiated to beating cardiomyocytes (iPSC-CMs), and hypertrophic growth was induced via Endothelin-1 (ET-1) treatment together with siRNA mediated silencing of FTO. I observed that cell growth was attenuated upon silencing of FTO and expression of the stress-marker ANP was reduced.

To determine, how the demonstrated in-vitro effect presents in vivo, I bred and characterized a cardiomyocyte specific Fto knockout (KO) mouse and applied the PO model to investigate hypertrophy and heart failure in FTO-deficient mice. Intriguingly, KO mice show a maladaptive response to $\mathrm{PO}$, with early onset of dilatation and significantly impaired cardiac function compared to control mice. Therefore, I hypothesize that the attenuated hypertrophy observed in vitro represents the inability of FTO-depleted CMs to undergo initial compensatory adaptation as seen in the in vivo model.

Together, my findings underline the importance of m6A RNA methylation in cardiac hypertrophy and heart failure progression and provide insight into the manipulation of FTO as a potential therapy in pressure overloaded hearts. 


\section{Introduction}

\subsection{Cardiovascular diseases}

Cardiovascular diseases (CVDs) are the most prevalent cause of death worldwide, with almost 18 million yearly fatalities which represent almost $31 \%$ of all global deaths (World Health Organization 2017). CVDs are comprised of a group of diseases of the heart and circulatory system, the most common being coronary arterial diseases (triggered by myocardial infarction), high blood pressure, arrhythmias and atrial fibrillation, heart valve obstructions and congenital heart defects (Flora and Nayak 2019). Cardiac remodeling occurs as a response to stress caused to the heart (Hill and Olson 2008; Azevedo et al. 2016; Nakamura and Sadoshima 2018).

\subsubsection{Cardiac remodeling}

Cardiac remodeling describes the molecular, cellular, and interstitial changes of heart tissue which manifest as changes in shape, sizes, and function of the heart, and which result from events such as cardiac injury, infarction, pressure or volume overload and other cardiomyopathies (Azevedo et al. 2016).

Typically, an increase in hemodynamic stress or load leads to hypertrophic changes of the heart. One type of hemodynamic stress is increased afterload, also called pressure overload (PO) (Norton et al. 2002; Toischer et al. 2010). In PO, the heart needs to work against a stronger force during the systole when ejecting blood and occurs typically due to aortic stenosis (Rockman et al. 1991; Toischer et al. 2010; Burchfield et al. 2013; Merino et al. 2018), wherein the left-ventricular exit to the aorta is narrowed (Rogers 2013).

As a response to $\mathrm{PO}$, neuroendocrine stimulation stabilizes cardiac output by inducing vasoconstriction and by increasing contractility. On the cellular level, cardiomyocytes (CMs) undergo hypertrophy (HT), meaning a growth in size due to an increase in contractile units by incorporation of additional sarcomeres (Wilson et al. 2014). This leads to concentric hypertrophic growth resulting in increased left ventricular wall thickness, and the ventricular chamber diameter may decrease depending on the severity of the HT. In this adaptive, compensated state the cardiac function is largely preserved. However, persistent hemodynamic stress leads to a decompensation and ultimately to heart failure (HF) (Norton et al. 2002; Inamdar and Inamdar 2016). HF is a pathologic condition, in which the heart is no longer able to pump a sufficient amount of blood throughout the system. HF is the long-term outcome of a period of pathological remodeling (Figure 1.1). 


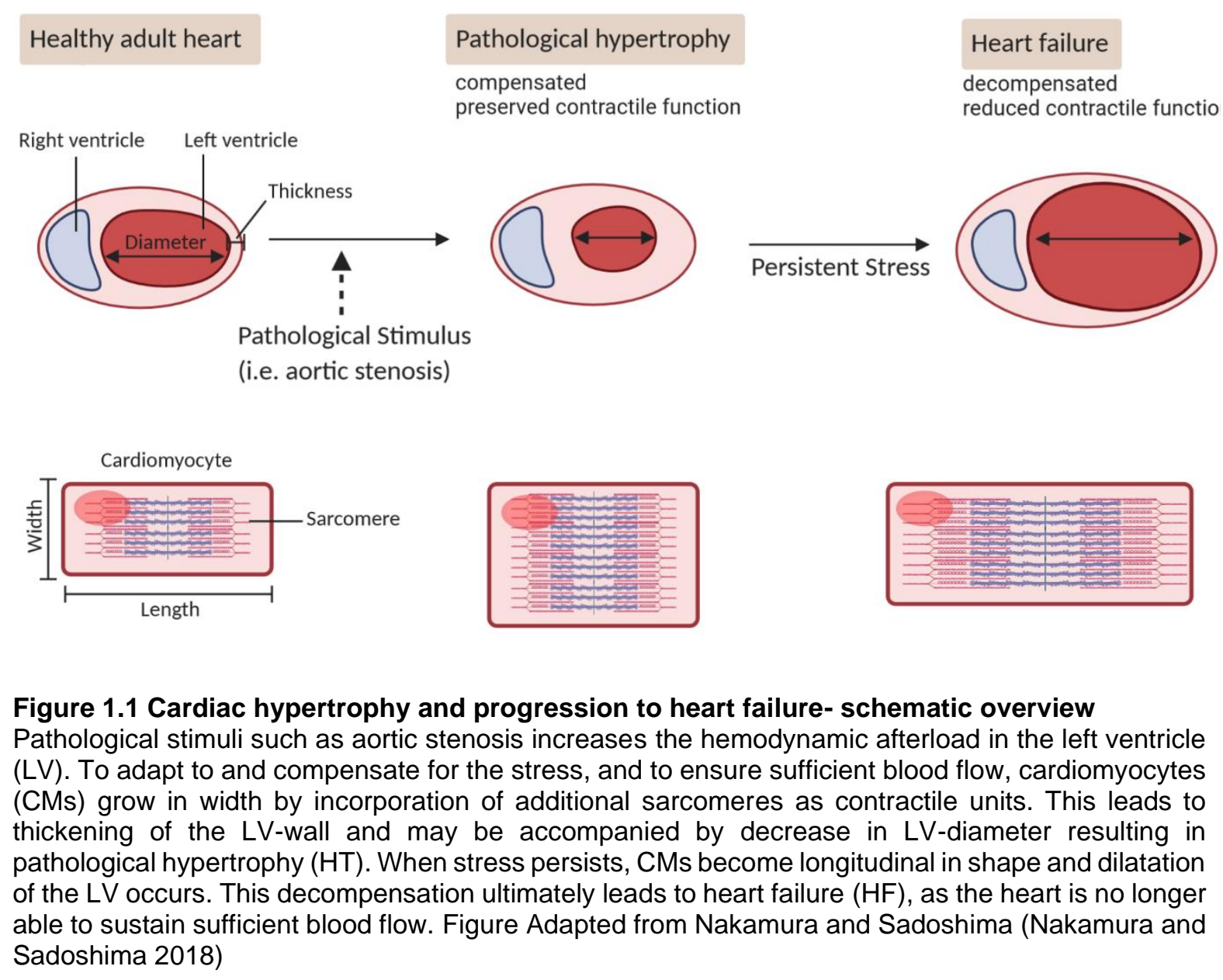

\subsubsection{Molecular mechanisms of cardiac remodeling}

On the molecular level, cardiac remodeling is triggered by neuroendocrine hormones and mechanical forces. For example, angiotensin II (AT), endothelin-1 (ET-1) and catecholamines are secreted in response to cardiac stress like PO. By binding to their respective receptors, signaling cascades get activated influencing contractility and gene expression. Catecholamines induce protein kinase A (PKA), AT and ET-1 activate protein kinase Ca (PKCa) and calcium/calmodulin dependent-protein kinase type II (CamKII). PKA, PKCa and CamKII further regulate $\mathrm{Ca}^{2+}$ handling proteins like ryanodine receptor 2 (RYR2), phospholamban (PLN) or sarcoplasmic reticulum $\mathrm{Ca}^{2+}$ ATPase (SERCA), thereby increasing contractility (Zhang et al. 2003; van Berlo et al. 2013; Newton et al. 2016; Nakamura and Sadoshima 2018). Neuroendocrine hormones further activate $\mathrm{G}$ proteins in CMs which in turn induce mitogen-activated protein kinase (MAPK) signaling pathways leading to the expression of pro-hypertrophic genes via myocyte enhancer factor 2 (MEF2), GATA binding protein 4 (GATA4) or nuclear factor of activated T-cells (NFAT) acting as transcription factors (Rose et al. 2010; Nakamura and Sadoshima 2018). These pro-hypertrophic genes, such as actin alpha 1 (ACTA1) or myosin heavy chain 7 (MYH7) are usually not expressed in adult cardiac tissue 
but are found in developing hearts. Therefore, it is called re-expression of the fetal gene program (Frey and Olson 2003; Hill and Olson 2008) and the translated proteins can serve as biomarkers for detection of cardiac remodeling.

Furthermore, neuroendocrine hormonal stimulation leads to mitochondrial dysfunction which increases reactive oxygen species (ROS) level. This leads to reduced contractility caused by increased apoptosis of CMs, stiffening of the tissue by fibrosis and impaired Ca2+ handling accompanying the mitochondrial dysfunction (Hill and Olson 2008; Toischer et al. 2010; van Berlo et al. 2013; Nakamura and Sadoshima 2018). Also energy metabolism is impaired during progression to heart failure where a switch from fatty acid to glucose metabolism takes place (Azevedo et al. 2016).

Upon stretch due to the pathologic hypertrophic growth, atrial natriuretic peptide (ANP, gene $N P P A)$ and brain natriuretic peptide (BNP, gene $N P P B)$ are released by CMs which activate protein kinases $\mathrm{G}$ (PKG) in an auto- or paracrine manner. PKGs inhibit further cell growth (Nakamura and Sadoshima 2018) and hence progression to HF with dilatation of the ventricle occurs marked by an increase in ventricular diameter.

\subsubsection{Therapeutic options for treatment of heart failure}

Available pharmacotherapies to treat heart failure mainly concentrate on targeting neurohormonal signaling pathways. For example, angiotensin-converting-enzyme (ACE) inhibitors block the conversion of angiotensin I to angiotensin II, which in turn prevents vasoconstriction, relaxing the vasculature and therefore reducing the hemodynamic load on the failing myocardium (Shah et al. 2017). Another common therapeutic strategy is the use of beta-adrenergic receptor blockers (B-blockers), which inhibit the renin angiotensin system (RAAS) reducing cellular stress by adrenaline and therefore lowering blood pressure and heart rate which would otherwise accelerate pathological remodeling (Shah et al. 2017). However the efficacy of these therapeutic approaches is limited, as mortality remains high in heart failure patients (Benjamin et al. 2018). Some preclinical and clinical studies focus therefore on the inhibition and manipulation of other signaling pathways commonly deregulated in $\mathrm{HF}$, such as MAPK- kinases or CaMKII (van Berlo et al. 2013). As these therapies concentrate mainly on single targets, these approaches have limited success given the complexity of the signal cascades deregulated in pathological remodeling. Some therapies may even be disadvantageous, as it has been shown that during the manipulation of complex signaling networks transcription factors such as NFAT, MEF2 or GATA4 may become activated, ultimately promoting pathologic gene expression (Hill and Olson 2008; van Berlo et al. 2013). 
Pharmacotherapies mainly concentrate on targeting cell signaling pathways, but it was shown that in cardiac remodeling and progression to heart failure not only cellular signaling or the transcriptome is affected. The epitranscriptome is deregulated in heart failure progression as well (Anand et al. 2013; Gilsbach et al. 2018; Prasher et al. 2020), adding an additional layer of molecular regulation and therapeutic targeting potential.

\subsection{Epigenetics in heart failure}

Aberrant gene expression, such as re-expression of the fetal gene program, is a hallmark of the progression to HF (Frey and Olson 2003; Hill and Olson 2008). There is increasing evidence, that gene expression deregulation is orchestrated by transcription factors and epigenetic processes (Prasher et al. 2020). Epigenetic processes include DNA and histone modifications or the expression of non-coding RNAs. It has been shown, that DNA methylation may be important during maturation of the heart (Gilsbach et al. 2018) and that histone modifications influence the transcription of cardio-specific gene programs (McKinsey and Olson 2005; Anand et al. 2013; Haldar and McKinsey 2014; Gilsbach et al. 2018). However, utilizing these epigenetic processes for diagnosis of CVDs is limited (Soler-Botija et al. 2019) and therapeutic options are scarce due to lack of specific and selective inhibitors (Haldar and McKinsey 2014). Therefore, other potential strategies for treatment of heart failure need to be explored, like the less studied epigenetic process of RNA methylation.

\subsubsection{RNA methylation}

Not only DNA can be modified by epigenetic marks, but RNA can be modified epigenetically as well. Many RNA modifications have been identified and include for example methylation at different positions of nucleosides, such as 5-methylcytosine ( $\mathrm{m} 5 \mathrm{C})$, 2-methyladenosine (m2A), or N6-methyladenosine (m6A) (Zaccara et al. 2019). The global distribution of these RNA modifications is called the epitranscriptome and has been intensively studied in various diseases (Engel et al. 2018; Widagdo and Anggono 2018; Chen et al. 2019; Han et al. 2020; Liu et al. 2020), as it adds a new layer of molecular signaling and control of cellular functions.

The most prevalent RNA modification is N6-methyladenosine (m6A) (Zaccara et al. 2019; Zhang et al. 2019), in which the $6^{\text {th }}$ amine group of adenosine is methylated. This modification was first described in the 1970s (Desrosiers et al. 1974; Perry et al. 1975) in eukaryotic messenger RNA (mRNA), but long thought to be of minor relevance. Nowadays it is known, that m6A is present in all classes of RNA, such as mRNA (Rottman et al. 1974), ribosomal RNA (rRNA) (Dominissini et al. 2012), transfer RNA (tRNA) (Blanco et al. 2014), small nuclear RNA (snRNA) (Linder et al. 2015), micro RNA (miRNA) (Berulava et al. 2015a) and long non- 
coding RNA (IncRNA) (Fu et al. 2014; Patil et al. 2016). m6A RNA methylation is dynamic and reversible (Fu et al. 2014; Roundtree et al. 2017; Yang et al. 2018), and is mediated by RNA methylases ('writer') and RNA demethylases ('eraser') and can be recognized by 'reader'proteins (Figure 1.2).

With the development of new techniques and methods to analyze RNA methylation such as methylated RNA immunoprecipitation sequencing (MeRIP-seq) (Dominissini et al. 2012; Meyer et al. 2012), the m6A epitranscriptome was mapped for the first time in 2012. It was observed that $\mathrm{m} 6 \mathrm{~A}$ methylation marks are enriched in specific mRNAs consistently throughout different cell lines and tissues (Schwartz et al. 2014; Linder et al. 2015). Further, the consensus motif DRACH ( $D=A, G$ or $U ; H=A, C$ or $U$ ) was identified as the main site of methylation (Dominissini et al. 2012; Meyer et al. 2012; Schwartz et al. 2014). While m6A peaks were found to be enrichment mainly in the 3' untranslated region (3' UTR) of transcripts and around stop codons (Dominissini et al. 2012; Meyer et al. 2012), methylation marks were also found throughout the whole mRNA transcript including the coding sequence (CDS) and the 5' UTR (Meyer et al. 2015). Some mRNA transcripts carry only single m6A modifications, while others can carry more than 20 marks at different positions (Dominissini et al. 2012; Meyer et al. 2012; Linder et al. 2015), but on average one to three m6A marks are found in methylated transcripts (Zaccara et al. 2019) . To which extent transcripts carry m6A marks and how they are targeted for methylation is still poorly understood and the subject of ongoing research. First studies published about m6A modifications identified the presence of long exons, meaning exons which are significantly longer than the average of $\sim 140 \mathrm{bp}$, to be a strong inducer of $\mathrm{m} 6 \mathrm{~A}$ addition in transcripts (Dominissini et al. 2012; Batista et al. 2014; Ke et al. 2015). However, as transcripts lacking this feature are also methylated, exon length is not the only trait regulating RNA methylation (Dominissini et al. 2012; Batista et al. 2014; Ke et al. 2015). Mapping studies have found that stably expressed genes, such as housekeeping genes or ribosomal proteins, carry fewer m6A marks (Schwartz et al. 2014), whereas transcripts with enriched m6A methylation code for processes regulating development, proliferation and cell fate (Dominissini et al. 2012; Meyer et al. 2012; Geula et al. 2015).

\subsubsection{Reading and writing the $\mathrm{m} 6 \mathrm{~A}$ code - dynamic and reversible}

It was shown that m6A methylation changes in stress (Engel et al. 2018) and disease conditions in different tissues (Chen et al. 2019; Han et al. 2020). This dynamic nature of RNA methylation derives from the interplay of methyltransferases and demethylases, facilitating addition and removal of m6A marks on RNA. Due to this ability to either 'write' or 'erase' methylation marks, they are also referred to as 'writer' and 'eraser' proteins (Yang et al. 2018; Zaccara et al. 2019) (Figure 1.2). 
The m6A modification is added to an adenosine by a writer complex consisting out of several subunits of different Methyltransferase Like Proteins (METTL). A METTL3-METTL14 heterodimer writes m6A on mRNA and other RNA polymerase II-derived transcripts (Śledź and Jinek 2016; Wang $P$ et al. 2016; Wang $X$ et al. 2016). METTL3 is the enzymatic subunit catalyzing the methylation process and METTL14 acts as an allosteric activator binding the RNA. Wilms tumor associated protein (WTAP) joins the heterodimer and assists in anchoring the complex to RNA (Ping et al. 2014). This writer complex transfers a methyl group from the donor substrate S-adenosyl methionine (SAM) to the adenine base (Śledź and Jinek 2016). It is important to note that other writer complexes exist. A METTL5-TRMT112 complex, for example, methylates a single m6A site in 18S rRNA (van Tran et al. 2019). Another methyltransferase, ZCCHC4, transfers a single m6A to 28S rRNA (Ma et al. 2019; van Tran et al. 2019). snRNA is methylated by METTL16, which also methylates some other non-coding RNAs (Warda et al. 2017).

Demethylases remove ('erase') the m6A methylation marks. Two RNA demethylases have been identified. The fat mass and obesity related protein (FTO) (Gerken et al. 2007), and AlkB Homolog 5 (ALKBH5) (Zheng et al. 2013) both remove m6A marks via oxidative demethylation. However, ALKBH5 was shown to be mainly enriched in testes and to have no effect on development or physiology except for defects in spermatogenesis (Zheng et al. 2013).

FTO was long thought to be the main m6A RNA demethylase (Jia et al. 2011). FTO is homologous to other demethylases of the ALKB family, but has only weak activity towards methylated DNA (Gerken et al. 2007). Furthermore, it was shown that FTO exhibits specificity towards m6A methylated RNA (Jia et al. 2011), strongly suggesting that m6A was the main substrate catalyzed by FTO.

Later studies proposed that m6A may not be the bona fide substrate of FTO as a conditional Fto-knockout $(\mathrm{KO})$ in the mouse brain did not result in increased m6A methylation (Hess et al. 2013). The independence of m6A levels from FTO was later described in other Fto-KO studies of murine embryos and cells (Mauer et al. 2017). Recent studies suggest that $\mathrm{N}^{6}, 2^{2}-\mathrm{O}$ dimethyladenosine (m6Am), another epigenetic RNA modification, may be the main substrate of FTO as indicated by a higher substrate specificity (Mauer et al. 2017; Mauer et al. 2019). However, the influence of FTO on m6A levels is still under debate, as other literature describes m6A level increase upon Fto-KO. For example, increased m6A levels were observed upon FTO depletion in leukemia (Li Z et al. 2017) and HeLa cells (Jia et al. 2011). 


\subsubsection{The various RNA methylation mediated effects - reading and interpreting the epitranscriptome}

m6A methylation is thought to have an influence on various cellular mechanisms (Yang et al. 2018; Zaccara et al. 2019) such as splicing (Xiao et al. 2016; Zhou et al. 2019), nuclear export (Roundtree et al. 2017), mRNA stability (Du et al. 2016; Huang et al. 2018), miRNA-binding (Berulava et al. 2015a), translation (Li A et al. 2017), as well as RNA-protein interactions (Liu et al. 2017; Perez-Perri et al. 2018).

The information encoded by m6A modifications is utilized and mediated by so-called 'reader' proteins. It can be discriminated between direct and indirect readers (Zaccara et al. 2019) (Figure 1.2). Direct readers are RNA binding proteins (RBPs) which specifically detect m6A marks, thus mediating methylation-induced processes. Indirect readers are influenced by m6A changes indirectly by for example tertiary structure changes induced by m6A marks, termed 'm6A-switch'.

The YTH-domain-containing protein family has been identified as direct m6A readers, with their YTH domain binding to RNA (Zhu et al. 2014). The YTH family is comprised of many members that show cell compartment specificity. YTHDC1 (DC1), for example, is located in the nucleus (Hartmann et al. 1999), whereas YTHDC2 (DC2) is located in both the nucleus and cytosol (Wojtas et al. 2017), while YTHDF1, YTHDF2 and YTHDF3 (DF1-3) are found in the cytosol (Du et al. 2016; Shi H et al. 2017; Shi et al. 2018). These different readers all mediate specific modes of molecular regulation.

The nuclear localized DC1 is thought to mainly influence splicing (Xiao et al. 2016), as it was shown to interact with splicing regulators (Hartmann et al. 1999) such as SAM68, SC35 or SRSF1 (Imai et al. 1998). As mRNA can already be m6A methylated in the nucleus (Bokar et al. 1997; Zheng et al. 2013; Meyer and Jaffrey 2017), this enables binding of DC1, mediating splicing. DC2, found in both the nucleus and cytosol, is expressed primarily in the testes (Bailey et al. 2017; Hsu et al. 2017). It has been shown to contain a helicase like domain and to regulate translation (Wojtas et al. 2017; Jain et al. 2018).

The reader proteins DF1-3 are paralogues of each other (Patil et al. 2018). These proteins lack typical protein domains, but rather contain low-complexity regions (LCR) (Patil et al. 2018), which are protein regions with an unusually high number or overrepresentation of specific amino acids (Kumari et al. 2015). This LCRs enable them to participate in a mechanism termed liquid-liquid phase separation (LLPS) (Ries et al. 2019). LLPS is the condensation of RNA and proteins into dense phases resembling liquid droplets, forming structures such as stress granules, P-bodies, or RNA granules. The role of these membraneless cellular compartments is largely unknown, but it is hypothesized that mRNA can be stored in these droplets, protected 
from degradation, stabilized for transport, or secluded from ribosomes, thereby affecting translation (Brengues et al. 2005; Bhattacharyya et al. 2006). Conversely, an opposite role has been hypothesized suggesting instead that these droplets may mediate mRNA decay (Buchan and Parker 2009; Protter and Parker 2016). The property of m6A-reader proteins DF1-3 to undergo phase-separation due to their low-complexity domain is especially interesting in epitranscriptomic regulation, as it has been shown, that binding of DF1-3 to methylated RNA enhanced LLPS significantly (Ries et al. 2019). As m6A-RNA has been shown to be recruited to DF1-3 to a higher extent than unmethylated RNA, hence inducing phase separation, this mechanism can regulate manifold processes such as RNA transport, storage, or decay in an m6A-regulated manner.

In contrast to the direct readers of the YTH family, indirect readers do not recognize methylated RNA, but methylation-dependent conformational changes of transcripts. Methylated adenosine can pair with Uracil bases in a manner, similar to A-U base pairing in RNA but to a weaker extent (Roost et al. 2015), lowering the melting temperature by $\sim 5^{\circ} \mathrm{C}$. Thus, m6A reduces the stability of RNA tertiary structures near m6A consensus motifs leading to unfolding and linearization, a process termed the 'm6A switch' (Liu et al. 2015). This allows for better access of RBPs to the single stranded RNA, as has been observed with the RBP HNRNPC (Liu et al. 2015). HNRNPC is responsible for the processing of pre-mRNA and participates in splicing. Due to the $\mathrm{m} 6 \mathrm{~A}$-induced change in structure, the function of this protein is indirectly regulated by the methylation, thereby acting as an indirect reader. This m6A-switch potentially affects all RBPs with consensus motifs near m6A marks, and indeed some studies have shown weaker binding affinity of i.e. HNRNPG (Liu et al. 2017), affecting splicing, or HNRPA2B1 (Wu et al. 2018), affecting miRNA processing. Until now it is not known, if this effect is mediated by a direct m6A-protein interaction or solely by the m6A-switch (Alarcón et al. 2015; Wu et al. 2018).

A third potential role of m6A methylation may be mediated by 'anti-readers', RBPs which are repelled by $\mathrm{m} 6 \mathrm{~A}$ marks. The 'anti-readers' may include proteins such as 63BP1, partaking in stress granule formation and FMR1, functioning as a translational repressor (Edupuganti et al. 2017) or LIN28A, participating in miRNA processing (Sun et al. 2019). Furthermore, it has been shown, that ribosomes stall at m6A marks in bacteria (Choi et al. 2016) preventing translation elongation. Yet, it remains unknown if such an effect is also present in eukaryotic cells, even if first evidence by ribosomal profiling suggest this possibility (Choi et al. 2016). 


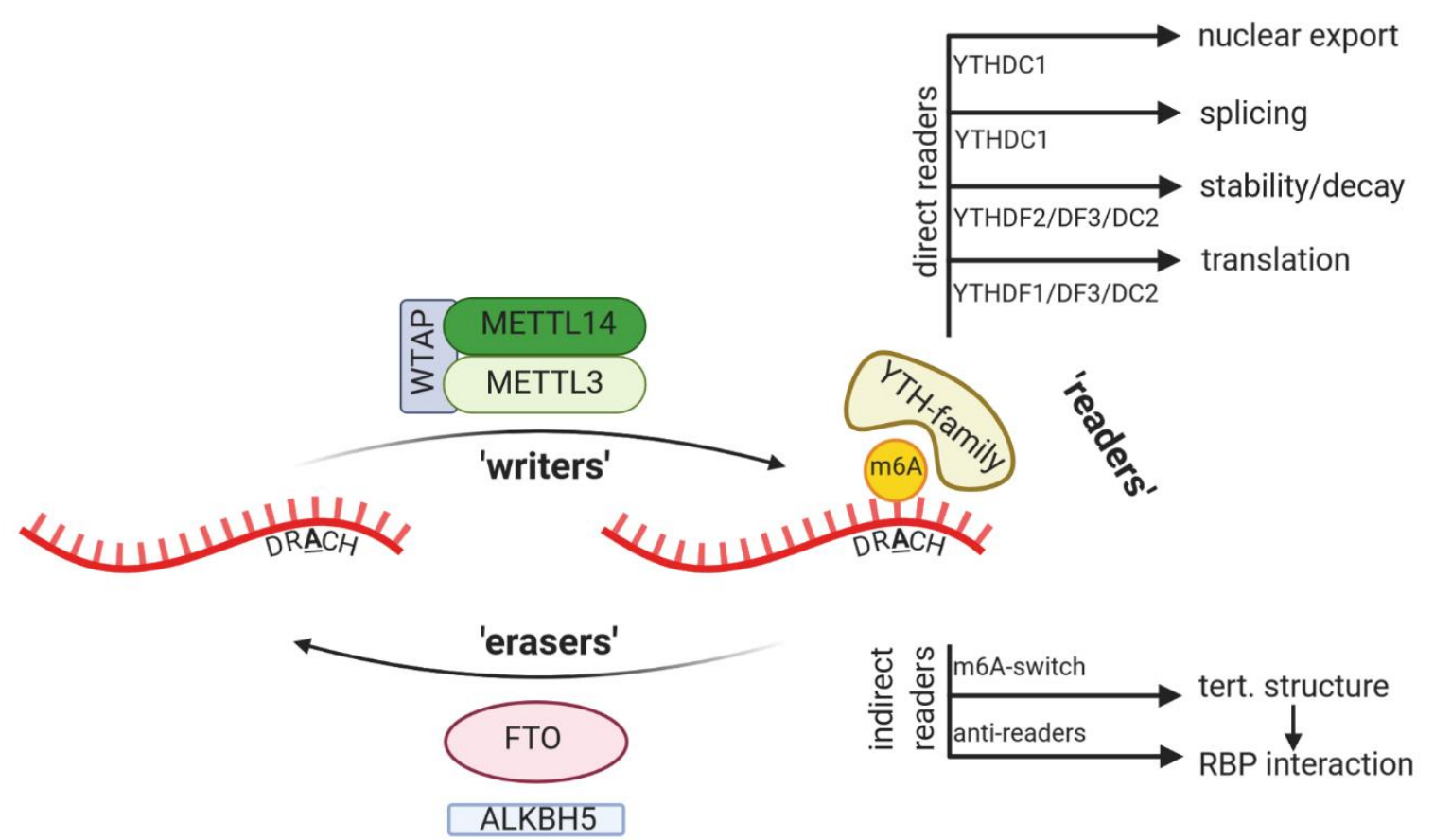

Figure 1.2 Dynamic and reversible m6A RNA methylation

RNA can be m6A methylated dynamically and reversibly, mainly at the DRACH consensus motif. The 'writer' complex METTL3-METTL14, assisted by WTAP, adds the m6A-mark to RNA. METTL3 is the catalytic subunit and acts as a methyltransferase, while METTL14 acts as an allosteric activator, binding to the RNA and assisting METTL3. WTAP stabilizes the heterodimer. FTO and ALKBH5 remove the m6A-mark by oxidative demethylation, acting as 'erasers'. m6A methylation marks can be identified by 'reader' proteins of the YTH-family affecting nuclear export, splicing, RNA stability or translation. m6A RNA methylation can alter the tertiary structure of RNA ('m6A-switch') influencing access and binding of RBPs. 


\subsubsection{RNA methylation in the heart}

Although it has been studied extensively in cancer and neurological diseases, little is known about the role of RNA methylation in cardiac diseases. Recently, first results were reported on $\mathrm{m} 6 \mathrm{~A}$ methylation in the heart. $\mathrm{m} 6 \mathrm{~A}$ methylation in cardiac ischemia was found increased in hypoxic, ischemic regions, whereas $\mathrm{m} 6 \mathrm{~A}$ was absent in the peri-infarction zone (Mathiyalagan et al. 2019). Mathiyalagan and colleagues described that FTO selectively demethylated transcripts coding for cardiac contractile proteins and hypothesized that this would prevent degradation of transcripts and hence improve protein expression under hypoxic conditions as found in ischemia. Another study described an increase of $\mathrm{m} 6 \mathrm{~A}$ in response to hypertrophic stimuli in primary cell cultures, and methylated transcripts were found to be coding for kinases and signaling pathway proteins (Dorn et al. 2019). A comparable study (Kmietczyk et al. 2019) also found that mainly signal transduction and transcription regulation pathways were affected by RNA methylation in cardiac tissues. Dorn et al. further described that METTL3 overexpression (OE) and therefore $\mathrm{m} 6 \mathrm{~A}$ increase stimulates hypertrophic responses and attenuates cardiac dysfunction in a PO mouse model, whereas a knockout (KO) of METTL3 negatively influences cardiac homeostasis leading to maladaptive eccentric remodeling (Dorn et al. 2019). They hypothesize that m6A impacts the cell shape and geometry of CMs and conclude that $\mathrm{m} 6 \mathrm{~A}$ is necessary for cardiac hypertrophy to occur. Another study, focused on METTL3 manipulation by KO or OE, also concludes that changes in m6A upon METTL3 manipulation influence mRNA stability and translation thereby regulating growth control of CMs in response to stress (Kmietczyk et al. 2019). 


\subsection{Objectives}

The field of epigenetics is studied extensively in various cell types and diseases, as it adds new layers of molecular regulation beyond post-translational modifications and posttranscriptional control. Especially the role of DNA and histone modifications were studied in the heart in the past years (Backs and Olson 2006; Gilsbach et al. 2014; Haldar and McKinsey 2014; Gilsbach et al. 2018). However, the effect and impact of RNA methylation, especially of the m6A RNA modification in cardiovascular diseases, is less studied. Only recently have a few studies been published (Dorn et al. 2019; Kmietczyk et al. 2019; Mathiyalagan et al. 2019) which prove the spark of interest to investigate this additional level of molecular regulation. When this study began, almost nothing was known about RNA methylation in the heart. The epitranscriptome of the heart had never been described on a global level and published studies focused on RNA methylation in ischemic and hypoxic conditions (Mathiyalagan et al. 2019) or on manipulation of the m6A writer METTL3 (Dorn et al. 2019; Kmietczyk et al. 2019).

The aim of my doctoral studies was to investigate the effect of m6A methylation in cardiac hypertrophy and heart failure. Furthermore, I tried to elucidate the role of FTO and provide insight into the effect of manipulation of the RNA-methylation machinery by FTO depletion in cardiac hypertrophy and heart failure. Therefore, the aims of my thesis were to:

1. Describe the epitranscriptome in mouse and human heart tissue.

I analyzed the global epitranscriptome of mouse and human heart tissue by NGS and MeRIPseq to provide insight into $\mathrm{m} 6 \mathrm{~A}$ methylation in the heart.

2. Analyze methylation changes in cardiac tissues in response to hypertrophy and in the heart failure state in human and mice.

I used a transverse aortic constriction (TAC) model in mice to induce hypertrophy and heart failure as well as tissue from failing human hearts to elucidate RNA methylation changes in response to the disease condition via NGS and MeRIP-seq analysis.

3. Establish a hypertrophy cell culture model.

I differentiated human induced pluripotent stem-cells (iPSCs) into beating cardiomyocytes (iPSC-CMs), which were further treated with ET-1 to induce hypertrophic growth.

4. Establish a knockdown of the demethylase FTO in iPSC-CMs to provide first insight into potentially altered hypertrophic response.

I analyzed a potential effect of siRNA mediated knockdown of the m6A demethylase FTO in the iPSC-CMs on cell growth by immunofluorescence microscopy as well as by immunoblotting of the typical hypertrophic marker ANP. 
5. Generate and characterize a cardiomyocyte specific knockout $(\mathrm{KO})$ in mice.

To test the potentially altered growth response obtained in vitro by cell culture in vivo, I generated a cardiomyocyte specific $\mathrm{FTO}^{\mathrm{ckO}}$ mouse by cre-mediated excision of exon 3 of Fto and validated the successful KO on all molecular levels by legacy PCR, RT-qPCR and Western blotting. Furthermore, I described the basal phenotype by monitoring survival and echocardiographic analysis.

6. Evaluate the response of the newly generated mice to TAC-induced pressure overload and subsequent hypertrophy and heart failure.

To analyze altered responses of Fto ${ }^{\mathrm{ckO}}$ mice hearts to TAC induced PO, I assessed the cardiac performance and dimension of the mouse hearts by echocardiography. 


\section{Methods}

\subsection{Human tissue sampling}

All experiments on human tissue were approved by the institute's ethical committee and followed the principles outlined in the Declaration of Helsinki. Patients provided written informed consent for the use of cardiac tissue samples in research.

LV tissue was sampled from freshly explanted hearts from end-stage HF patients during cardiac transplantation as a result from ischemic or dilated cardiomyopathies (ICM and DCM, respectively). In ICM samples, the tissue was taken from the non-ischemic, peri-infarction area to omit hypoxic scar tissue, so the tissue sections are comparable to DCM samples all being of end-stage heart failure condition (HF). Detailed patient information is given in the Appendix Table 5.2. Healthy human hearts from donor patient with no preexisting cardiac disease or noncardiac related reasons of death served as non-failing (NF) control tissue. Those hearts were initially planned for transplantation but were rejected by the surgeon due to for example organ mismatch, local hypokinesia or elevated C-reactive protein levels. Information for healthy control patients are given in the Appendix Table 5.1.

\subsection{Animal studies}

All animal experiments in this project were approved and conducted following institutional and governmental regulations and in accordance with the ethical standards laid down in the Declaration of Helsinki 1964 and conforms to the Guide for the Care and Use of Laboratory Animals (Institute of Laboratory Animal Resources and National Research Council 1996). Animals were maintained under standard husbandry with regulated 12 hours light/dark cycle and access to food and water ad libitum. FVB/N mice were delivered by Charles River, all other mouse strains were bred in-house.

\subsubsection{Generation of mouse strains}

A cardiomyocyte specific knockout (CKO) mouse strain was bred for this study. In this strain, the Fto-gene expression was depleted via cre-recombinase mediated excision of exon 3 of the gene flanked by loxP sites.

To first create Fto-flox mice, the B6Dnk;B6Brd;B6N-Tyrc-Brd Ftotm1a(EUCOMM)Wtsi/WtsiCnbc strain (Wellcome Trust Sanger Institute) was crossed with the C57BL/6N-Tg(CAGFlpo)1Afst/Mmucd strain (Kranz et al. 2010) strain, to remove the lacZ reporter cassette. The 
remaining Flpo allele was outcrossed by back-crossing the obtained mice with C57BL6/N wtmice resulting in Flox-mice where exon 3 of Fto is flanked by loxP sites (Fto $\left.{ }^{\mathrm{fl} / \mathrm{f}}\right)$.

To generate the $\mathrm{cKO}$, male Fto ${ }^{\mathrm{f} / \mathrm{fl}}$ mice were mated with $\alpha M H C$-Cre females ( $\alpha M H C$-Cre, Jackson no. 011038, C57BL/6N and C57BL/6J mixed background) (Agah et al. 1997) bearing a cardiomyocyte specific cre-recombinase under the aMHC- promotor. The resulting heterozygous $\alpha \mathrm{MHC}-\mathrm{Cre}^{+} ; \mathrm{Fto}^{\mathrm{fl} / /+}$ mice (cre+ $\mathrm{fl} /+$ ) were further backcrossed with $\mathrm{Fto}^{\mathrm{fl} / \mathrm{fl}}$ mice to generate a homozygous Fto knock-out $(\mathrm{cre}+\mathrm{fl} / \mathrm{fl})$. These animals were used for experiments and are further called Fto ${ }^{\mathrm{ckO}}$.

$\alpha M H C-\mathrm{Cre}^{+}$mice $(\mathrm{cre}++/+)$ served as wildtype control and are further named Cre ${ }^{\text {Control }}$. Instead of using mice from the already existing $\alpha M H C-C r e^{+}$parental strain, a new strain was bred by backcrossing $\alpha M H C-C r e^{+}$; Fto ${ }^{f / f l}$ mice $(\mathrm{cre}+\mathrm{fl} / \mathrm{fl})$ with $\alpha M H C-C r e-$ mice $\left(\mathrm{cre}^{-}+/+\right)$until a homozygous $\alpha \mathrm{MHC}$-Cre ${ }^{+}$mice (cre $++/+$) strain was generated. This was done in order to rule out possible flox-site derived effects on the phenotype which may exist even if the loxP-sites were outcrossed. $\alpha M H C-C r e ; \mathrm{Fto}^{\mathrm{ff} / / \mathrm{ll}}$ mice (cre- $\left.\mathrm{fl} / \mathrm{fl}\right)$ mice, littermates of the $\alpha M H C-\mathrm{Cre}^{+} ; \mathrm{Fto}^{\mathrm{f} / / \mathrm{fl}}$ (cre+ fl/fl) mice served as another wildtype control to check for cre- recombinase derived effects and are further called Flox ${ }^{\text {Control. }}$

\subsubsection{Genotyping}

Mice were genotyped using primers for FTO and cre-recombinase (Table 2.1) with the MangoTaq ${ }^{\mathrm{TM}}$ Polymerase (Bioline; \#BIO-21078). Genomic DNA (gDNA) was isolated from tail biopsies by digestion in $200 \mu$ l DirectPCR-Tail lysis buffer (Viagen Biotech; \#102-T) supplied with $200 \mu \mathrm{g} / \mathrm{ml}$ Proteinase $\mathrm{K}$ on a heating block at $55^{\circ} \mathrm{C}$ and $400 \mathrm{rpm}$ overnight. Prior to the PCR run, Proteinase $\mathrm{K}$ was inactivated for $45 \mathrm{~min}$ at $85^{\circ} \mathrm{C}$. Detailed PCR-mix and Amplification Protocol are given in Table 2.2 and Table 2.3.

Afterwards, the PCR reactions were separated by agarose gel electrophoresis in a $2 \%$ TBE (45 mM Tris-borate, 1M EDTA) buffered agarose gel supplemented with Midori Green Advance (Nippon Genetics; \#MG04) (5 $\mu \mathrm{l}$ pro $100 \mathrm{ml}$ gel) for $45 \mathrm{~min}$ at $120 \mathrm{~V}$ constant. PCR product bands stained by the Midori Green were visualized on a UV-light table and band sizes were determined from comparison with the 100 bp DNA ladder (Thermo Fischer; \#SM1153). 
Table 2.1 Genotyping Primer

\begin{tabular}{ll}
\hline Primer Name & Sequece 5'-3' \\
\hline FTO_genFOR & GACTTGGGGCAGGTTTTCAG \\
FTO_genREV & CCACTTCTCCCCTCCTTCAA \\
FTO_recombination_R & CAAAGGACTTAGCACTCTCGG \\
Myh6Cre_TG_F & ATGACAGACAGATCCCTCCTATCTCC \\
Myh6Cre_TG_R & CTCATCACTCGTTGCATCATCGAC \\
Myh6Cre_+Ctrl_F & CAAATGTTGCTTGTCTGGTG \\
Myh6Cre_+Ctrl_R & GTCAGTCGAGTGCACAGTTT \\
\hline
\end{tabular}

Table 2.2 Genotyping reaction mix

\begin{tabular}{ll}
\hline Reagent & Volume $[\mu \mathrm{l}]$ \\
\hline MangoTaq Buffer 5X & 5 \\
$\mathrm{MgCl}_{2}(\mathbf{5 0} \mathrm{mM})$ & 1.5 \\
\hline dNTPs $(100 \mathrm{mM})$ & 0.5 \\
Forward Primer $(\mathbf{1 0} \mathrm{pmol} / \mu \mathrm{l})$ & 1 \\
Reverse Primer $(10 \mathrm{pmol} / \mu \mathrm{l})$ & 1 \\
MangoTag Polymerase & 0.2 \\
$\mathrm{H}_{2} \mathrm{O}$ & 14.8 \\
gDNA & 1 \\
Total & 25 \\
\hline
\end{tabular}

Table 2.3 Genotyping protocol

\begin{tabular}{lll}
\hline Step & Temperature $\left[{ }^{\circ} \mathbf{C}\right]$ & Time \\
\hline Denaturation & 94 & $5 \mathrm{~min}$ \\
Amplification (x35 cycles) & 94 & $30 \mathrm{sec}$ \\
& 59 & $30 \mathrm{sec}$ \\
& 72 & $45 \mathrm{sec}$ \\
Elongation & 72 & $5 \mathrm{~min}$ \\
Hold & 8 & As needed \\
\hline
\end{tabular}




\subsubsection{Transverse aortic constriction}

In transverse aortic constriction (TAC), the aortic diameter is reduced via a knot tied around the aortic branch. This narrowed aorta leads to a pressure gradient against which the heart needs to pump blood, which induces pressure overload (PO) leading to cardiac hypertrophy (HT) and heart failure (HF) with progressing time. This intervention is a model for aortic stenosis (deAlmeida et al. 2010). For this study, 8-10 weeks old mice were randomly selected for either Sham or TAC surgery, with an average distribution between males and females in all groups.

In order to operate mice, animals were anaesthetized by injecting $75-100 \mu \mathrm{l} 0.9 \%$ sodium chloride $(\mathrm{NaCl})$ solution containing Medetomidin $(0.5 \mathrm{mg} / \mathrm{kg})$, Midazolam $(5 \mathrm{mg} / \mathrm{kg})$ and Fentanyl $(0.05 \mathrm{mg} / \mathrm{kg})$ intraperitoneal. The actual surgery was performed using a minimally invasive approach. The mouse heart was opened via a $1-1.5 \mathrm{~cm}$ long suprasternal incision and the aortic arch was exposed and prepped carefully free from the thymus and fat tissue. A 5-0 Polyviolene (non-absorbable) suture was placed between the first and second arch and two loose knots tied. For comparable and standardized constriction, either a 27-gauge needle or 26-gauge needle, depending on the experimental setup, was tied against the aorta, the knots fastened, and the needle removed. Sham animals underwent the same procedure except for banding of the aorta. The mouse was then closed using a 6-0 prolene suture. After the surgery, antagonization was achieved via subcutaneous injection of $50 \mu \mathrm{l}$ Atipamezol $(2.5 \mathrm{mg} / \mathrm{kg})$ and Flumazenil $(0.1 \mathrm{mg} / \mathrm{kg})$ dissolved in $0.9 \% \mathrm{NaCl}$ solution. Buprenorphin served for analgesia after the intervention and was applied subcutaneously in the common dose of $0.05-0.1 \mathrm{mg} / \mathrm{kg}$. Mice could recover in a heat cabinet for at least 24 hours. Further post-operative care included application of carprofen ( $5 \mathrm{mg} / \mathrm{kg}$ ) subcutaneously as analgesia for two consecutive days as well as daily monitoring and weighting the mice. One to three days after the surgery, the pressure gradient along the aorta was measured applying Doppler echocardiography (Section 2.2.4). Further echocardiographical measurements were taken 1, 4 and 8 weeks post-TAC and, depending on the experimental setup, hearts were isolated in the end for further molecular and histological analyses.

TAC-surgery was performed by Alessya Kretzschmar (Clinic for Cardiology and Pneumology), Sabrina Koszewa (Clinic for Cardiology and Pneumology) and Sarah Zafar (Clinic for Pharmacology and Toxicology).

\subsubsection{Echocardiography}

To assess the cardiac phenotype via heart function and remodeling changes of Sham and TAC operated animals, mice were examined with transthoracic echocardiography using the Vevo 
2100 system (Visualsonics). In order to employ ultrasound, mice were anaesthetized using $1.5 \%$ isoflurane applied through a respiratory mask and the thorax was depilated with hair removal cream. During echocardiographic measurements, the vital functions of mice such as heart rate, respiratory rate and body temperature were monitored to stay in the standard range.

2D guided M-mode images were recorded in the long-axis, as well as short axis view at a left mid-ventricular level, with the help of an MS-400 $30 \mathrm{Mhz}$ transducer (Visualsonics) (Pistner et al. 2010). To examine the initial success of the TAC surgery, the pressure gradient across the transverse aorta was measured by applying Doppler echocardiography using a MS250 20 Mhz transducer, to calculate the fractional flow reserve. Echocardiographic imaging was performed by the SFB1002 service unit team Roland Blume and Marcel Zoremba (Clinic for Pharmacology and Toxicology, Clinic for Cardiology and Pneumology) and Sabrina Koszewa (Clinic for Cardiology and Pneumology.

Recorded images were analyzed using the LV-trace function of the VevoLab software (Version 3.1.0, Visualsonics) in M-mode images. Cardiac performance, assessed by measuring heart dimensions, was analyzed determining the Anterior wall thickness (AwTh) and the left ventricular interdimensional diameter (LVID) in both the systole and diastole (;s, ;d). From these values the ejection fraction (EF) could be calculated automatically by the software. These parameters can be used to determine hypertrophic changes. The examination of the echocardiographic images was done in a blinded manner toward group assignment (Sham, TAC, cKO or Control). Statistical analysis of the echocardiographic data was performed in the GraphPad Prism Software (v. 8.4.2). Unpaired t-test was used for analysis of two groups (Sham vs. TAC) and two-way ANOVA for multiple comparison analysis (Sham/TAC and cKO/Control).

\subsubsection{Murine LV isolation}

For isolation of hearts and other tissues, mice were anesthetized with isoflurane and sacrificed by cervical dislocation. The thorax was opened, and the heart exposed. The heart was then cut out above the aorta and washed in petri-dish containing sterile $\mathrm{NaCl}$ solution by gently squeezing to eject remaining blood from the ventricles. The heart was then flushed with sterile $\mathrm{NaCl}$ by inserting a 21-gauge blunt needle into the aorta allowing for retrograde perfusion. The heart was weighted, the atria and right ventricle removed and the LV snap frozen in liquid nitrogen, either in whole or cut into smaller pieces, depending on the further experimental setup and methods to be applied. Furthermore, the tibia was isolated from fur and muscle tissue and its length measured, to later calculate heart weight/tibia length ratio to further assess hypertrophic changes. 


\subsubsection{Langendorff perfused heart cardiomyocyte isolation}

To separate cardiomyocytes from non-myocytes like fibrocytes and endothelial cells that make up heart tissue, hearts were Langendorff perfused and myocytes isolated as described by Toischer et al. (Toischer et al. 2017). For this, mouse hearts were isolated similar as described above (Murine LV isolation), but the heart was transferred immediately to a petri dish containing ice cold Ca-free Tyrode (Table 2.4) instead of sterile saline. A 20-gauge needle was inserted into the aorta and mounted to a Langendorff setup to be retrogradely perfused with Tyrode at a flowrate of $3.5 \mathrm{ml} / \mathrm{min}$ for $3 \mathrm{~min}$ at $37^{\circ} \mathrm{C}$. In the next step, the heart was digested by perfusing $7 \mathrm{~min}$ with digestion buffer (Tyrode supplemented with Liberase TM (Sigma, \#LIBTM-RO), trypsin 10X, 2.5\% and $10 \mathrm{mM} \mathrm{CaCl}_{2}$ ). When the heart became soft, the atria were excised, and the LVs were crushed in $2.5 \mathrm{ml}$ digestion buffer for further 30 seconds. The digestion reaction was stopped by adding $2.5 \mathrm{ml}$ Tyrode supplemented with $\mathrm{BCS}$ and $\mathrm{CaCl}_{2}$ (Stop Solution) and the cell suspension was homogenized by gently aspiration and pushout in a $1 \mathrm{ml}$ syringe without a needle. After $10 \mathrm{~min}$ of sedimentation of the cells, the resulting pellet was washed twice with Tyrode and finally snap-frozen in liquid nitrogen for later RNA or protein isolation.

Table 2.4 Tyrode Solution

\begin{tabular}{ll}
\hline Reagents & Concentration [mmol/L] \\
\hline $\mathrm{NaCl}$ & 113 \\
$\mathrm{KCl}$ & 4.7 \\
$\mathrm{KH}_{2} \mathrm{PO}_{4}$ & 0.6 \\
$\mathrm{Na}_{2} \mathrm{HPO}_{4} \times 2 \mathrm{H}_{2} \mathrm{O}$ & 0.6 \\
$\mathrm{MgSO}_{4}$ × $7 \mathrm{H}_{2} \mathrm{O}$ & 1.2 \\
$\mathrm{NaHCO}_{3}$ & 12 \\
$\mathrm{KHCO}_{3}$ & 10 \\
$\mathrm{HEPES}$ & 10 \\
Taurine & 30 \\
2,3-butanedione monoxime (BDM) & 10 \\
Glucose & 5.5 \\
Phenol red & 0.032 \\
\hline
\end{tabular}




\section{3 iPS-cell culture}

For in vitro studies of FTO and m6A mediated effects, human induced pluripotent stem cells (iPSCs) were used. Cell lines were a kind gift from Dr. Katrin Streckfuß-Bömeke (Clinic for Cardiology and Pneumology, University Medical Center Göttingen). Those cell lines were already reprogrammed and characterized by her group and published (Streckfuss-Bömeke et al. 2013; Borchert et al. 2017). Two different cell lines were used, one reprogrammed with a single cassette reprogramming vector termed STEMCCA (Sommer et al. 2012; StreckfussBömeke et al. 2013), one with episomal plasmids expressing transiently the reprogramming factors (Yu et al. 2011; Borchert et al. 2017). As the STEMCCA method makes use of a viral vector, an integration of vector-parts in the genome of the cells is possible, whereas the episomal plasmid method is a non-viral, integration-free method. Therefore, by using these two independent cell lines, potential effects seen in the experiments can be independently verified, allowing stronger statements on observations as cell line specific effects can be ruled out.

The two cell lines used were namely WT-D2-1U (WTD2) [STEMCCA method] and PCTRL1.1 ( $\mathrm{pCTRL}$ ) [episomal plasmid method]. All investigations follow the Institutes ethical and regulatory guidelines and were approved by the ethics committee. Cell donors have given written consent for the use of cells in scientific studies. Cell culture experiments were assisted by Nelly Wery von Limont and Alessa Pommeranz as part of their Medical Dissertation Project under my supervision.

\subsubsection{Standard culturing}

iPS- cells were cultured in 6-well plates. The wells were coated with Geltrex Matrix (Thermo Fisher; A1413302) for better cell adhesion. Cells were cultured in Essential8 ${ }^{\mathrm{TM}}$ Medium (Gibco; \#A1517001) (E8) at $37^{\circ} \mathrm{C}$ and $5 \% \mathrm{CO}_{2}$ in a humid environment. Medium was changed daily, and cells passaged every 3-4 days when they reached $\sim 80 \%$ confluency. For this, E8 medium was removed, cells washed once with $1 \mathrm{ml}$ Versene solution (Gibco; \#15040066), Versene was directly removed and cells incubated with $1 \mathrm{ml}$ fresh Versene at $37^{\circ} \mathrm{C}$ for $3-5 \mathrm{~min}$. Afterwards, Versene was aspirated, and the cells washed of the plate with $1 \mathrm{ml}$ E8 medium supplemented with $2 \mu \mathrm{M}$ Thiazovivin (TZV) (Millipore; \#420220). Cells were then transferred to new Geltrex coated wells in $2 \mathrm{ml}$ fresh E8 medium supplemented with $2 \mu \mathrm{M}$ TZV, with a splitting ration of $1: 8$ (125 $\mu$ l cell suspension) or $1: 10$ (100 $\mu$ l cell suspension). 


\subsubsection{Differentiation to cardiomyocytes}

Differentiation was performed in 12-well plates. Initially, cells were transferred during the passaging step in a 1:12 to 1:16 splitting ratio, depending on planned start of the differentiation. When cells reached $\sim 80 \%$ confluency, differentiation was initiated (Day 0 ) by a medium change from E8 to Cardiac differentiation medium (C.Diff) (an overview of growth media is given in Table 2.6) supplemented with $4 \mu \mathrm{M}$ CHIR99021 (Merck; \#361559), to induce WNTpathway activation. After 48 hours (Day 2), medium was changed to C.Diff medium containing 2.5 MM IWP2 (Merck; \#681671), a WNT inhibitor. This signaling manipulation induces differentiation of the cells into the cardiac lineage. 48 hours later (Day 4), medium was changed to C.Diff medium only, containing no supplements. Medium was changed with fresh C.Diff every second to third day until Day 15. A successful differentiation was indicated by spontaneous beatings of the cells around Day 10. Only wells with beating cells were further cultured. At Day 15 cells were re-plated in a lower density in Geltrex coated T-25 flask. Briefly, cells were washed with $0.5 \mathrm{ml}$ 0,25\%Trypsin-EDTA (Gibco; \# 2520056), Trypsin quickly removed, and cells incubated with $1 \mathrm{ml} /$ well fresh Trypsin at $37^{\circ} \mathrm{C}$ for $5 \mathrm{~min}$. The trypsin digest was stopped afterwards by transferring detached, digested cells into two volumes of Digestion Medium (refer to Table 2.6). The cell suspension was centrifuged at $200 \times \mathrm{g}$ for 5 minutes, supernatant removed, and cells resuspended in an appropriate volume Digestion Medium, usually $3 \mathrm{ml}$ for 12 wells with beating cells. $1 \mathrm{ml}$ suspension was then transferred to T-25 flasks containing $4 \mathrm{ml}$ Digestion Medium. Cells were left for 2-3 days to recover. After successful recovery, indicated by an attached, beating cell layer, cardiomyocyte selection took place. For that, Digestion medium was changed to Cardio Selection Medium (refer to Table 2.6), $4 \mathrm{ml} / \mathrm{T}$ 25 flask. As Selection medium contains lactic acid as a single energy source, noncardiomyocytes die, and only cardiomyocytes survive due to their ability to metabolize lactic acid. Selection took place for 4-5 days with medium change after two days. After selection, Medium was changed to Cardio Culture Medium (refer to Table 2.6) (4 ml/T-25 flask). Cells were then cultured for maturation to an age of at least 60 days (Day 60), with a Medium change every 3-4 days. Cells were then subsequently used in experiments (Section 2.3.6-2.3.11) or frozen (Section 2.3.3)

\subsubsection{Freezing of iPSC-CMs}

To avoid too much aging of the CMs, cells were frozen for later use. For cryo-conservation, cells were washed twice in DPBS and trypsinated for 5 minutes at $37^{\circ} \mathrm{C}$. Trypsination was stopped by transferring the detached cells into an equal volume of FBS (Gibco; \#;10270106) and subsequent centrifugation at $100 \times \mathrm{g}$ for $10 \mathrm{~min}$. After centrifugation, supernatant was 
discarded, and the cell pellet resuspended in FBS. The cell number was determined with the Casy Cell Counter (Innovatis). 5-10 mio. cells were frozen in Cryotubes in FBS supplemented with 10\% DMSO and 1\% TZV using an automated Kryo 560 freezer (Planer) (Freezing Protocol provided in Table 2.5)

Table 2.5 Freezing Protocol

\begin{tabular}{|c|c|c|c|c|}
\hline Step & $\begin{array}{l}\text { Start } \\
{\left[{ }^{\circ} \mathrm{C}\right]}\end{array}$ & Temperature & End Temperature $\left[{ }^{\circ} \mathrm{C}\right]$ & Rate $\left[{ }^{\circ} \mathbf{C} / \mathrm{min}\right]$ \\
\hline 1 & -1 & & -4 & -1 \\
\hline 2 & -4 & & -40 & -25 \\
\hline 3 & -40 & & -12 & +10 \\
\hline 4 & -12 & & -40 & -1 \\
\hline 5 & -40 & & -90 & -10 \\
\hline 6 & -90 & & -90 & Hold/Program end \\
\hline
\end{tabular}

\subsubsection{Re-plating cells for experiments}

Further experiments were performed in 6-well plates. For this, the differentiated, iPSC derived Cardiomyocytes (iPSC-CM) were re-plated from T-25 flask to 6-well plates. Cells were digested as described before. In brief, iPSC-CMs in T-25 flasks were digested with $3 \mathrm{ml}$ Trypsin, digestion stopped in digestion medium and cells centrifuged at $200 \times \mathrm{g}$ for $5 \mathrm{~min}$. Cells were then resuspended in an appropriate volume of digestion medium and counted with a Neubauer counting chamber. 600.000 cells per well of a 6 -well plate were seeded and left for recovery for at least 3 days.

\subsubsection{Recovery of cryo-conserved iPSC-CMs}

Alternately to re-plating matured cells for experiments, cells were also recovered from cryoconservation if needed. For thawing of cells, cryotubes were placed in a water bath at $37^{\circ} \mathrm{C}$ until thawn and cells directly transferred carefully dropwise into $10 \mathrm{ml}$ prewarmed Cardio Digest Medium in a $15 \mathrm{ml}$ Falcon tube. Cells were then centrifuged for $10 \mathrm{~min}$ at $100 \mathrm{xg}$, supernatant aspired and the cells pellet resuspended in an appropriate amount of Cardio Digest Medium and plated on T-25 flasks as described before. Recovered cells were again digested, counted and re-plated on 6-well plates with 600.000 cells/Well for further experiments. 
Table 2.6 Cell Culture Media

Component

Amount

Cardio Differentiation Medium (C.Diff.)

RPMI 1640 /w HEPES /w GlutaMax (Gibco; \#72400021)

Albumin, human recombinant

L-Ascobic Acid 2-Phosphate

filter to sterilize

$250 \mathrm{mg}$

$100 \mathrm{mg}$

\section{Cardio Culture Medium}

Cardio Differentiation Medium (C.Diff.)

B27 Supplement (Gibco; \#A17504044)

$500 \mathrm{ml}$

$10 \mathrm{ml}$

\section{Cardio Digestion Medium}

\begin{tabular}{l|l}
\hline \hline Cardio Culture Medium & $80 \mathrm{ml}$ \\
FBS & $20 \mathrm{ml}$ \\
TZV & $100 \mu \mathrm{l}$
\end{tabular}

Cardio Selection Medium

RPMI $1640 \varnothing$ HEPES $\varnothing$ GlutaMax (Gibco; \#11879020)

$500 \mathrm{ml}$

Albumin, human recombinant

$250 \mathrm{mg}$

L-Ascobic Acid 2-Phosphate

$100 \mathrm{mg}$

Lactate/HEPES

$2 \mathrm{ml}$

filter to sterilize

\subsubsection{ET-1 treatment}

For induction of stress and hypertrophic responses, iPSC-CMs were treated with Endothelin1 (ET-1) (Sigma; \#E7764) (Archer et al. 2017). A concentration of 3 nM ET-1 was determined to be effective (Section 3.2.2) and used subsequently. ET-1 Stock with a $10 \mu \mathrm{M}$ concentration (Stock 1) was prepared by dissolving powdered ET-1 in water. For application in cell culture, Stock 1 was further diluted to $1 \mu \mathrm{M}$ (Stock 2 ) with water. Stock 2 was diluted in Cardio Culture Medium, with $3 \mu \mathrm{l}$ ET-1/ml Medium giving rise to a final $3 \mathrm{nM}$ concentration which was then applied to the cells $(2 \mathrm{ml} /$ well) and incubated for 48 hours. Afterwards, cells were harvested for further molecular analysis. For cell harvesting, cells were washed twice with ice-cold DPBS and scraped off the plate with a cell scraper in $1 \mathrm{ml}$ ice-cold DPBS and centrifuged for $1 \mathrm{~min}$ at $13.000 \times \mathrm{g}$. Supernatant was discarded and the cell pellet snap-frozen in liquid nitrogen or used directly. 


\subsection{7 siRNA transfection}

A small interfering RNA (siRNA) mediated approach was used in vitro to induce a knockdown of FTO. siRNAs are 20-25 nt long double-stranded RNA molecules, that mediate effects via the RNA interference (RNAi) pathway. They can interfere with gene expression by binding to complementary mRNAs, inhibiting their translation into protein (Carthew and Sontheimer 2009; Zhang 2013). For this method, predesigned siRNAs complementary to sequences of the human FTO transcript were obtained from Qiagen. Five different siRNAs (Hs_FTO_5-8) were tested for their effectivity towards silencing of FTO and the best performing one, Hs_FTO_5 (Appendix Figure 5.3) was used in subsequent experiments. As negative control, scrambled siRNA with no RNA target was used. More information of the respective siRNAs is given in

Table 2.7.

Table 2.7 siRNA for FTO-KD in iPSC-CMs

Name of siRNA Target Sequence Catalog No.

$\begin{array}{lll}\text { Hs_FTO_5 } & \text { CAGGTCAGCGGTGGCAGTGTA } & \text { SI04177530 } \\ \text { Hs_FTO_6 } & \text { CTCCGAGAAGCCAGCAGTGTA } & \text { SI04180652 } \\ \text { Hs_FTO_7 } & \text { TAAGAGCAGAGCAGCATACAA } & \text { SI04293625 } \\ \text { Hs_FTO_8 } & \text { CAGGACGCTGAGAGAACTACA } & \text { SI04337039 }\end{array}$

AllStars Negative unknown 1027280

Control siRNA

siRNA was transfected with two different approaches. The first experiments for determination of suitable siRNA were performed using Lipofectamine Stem Transfection Reagent (Thermo Fisher; \#STEM00001). In brief, two reaction mixes were prepared (Table 2.8) and afterwards thoroughly mixed and incubated at RT for $10 \mathrm{~min}$. The final combined $150 \mu$ Reaction Mix was then added dropwise to the wells prepared with $1.5 \mathrm{ml}$ Opti-Mem medium (Gibco; \#31985062) and the cells incubated for 24 hours. After 24 hours, the transfection medium was changed to fresh Cardio Culture medium and cells harvested 24 hours after this medium change.

Table 2.8 Lipoefectamine Transfection Mix

\begin{tabular}{lcc}
\hline Component & Mix 1 $[\mu \mathrm{l}]$ & Mix 2 $[\mu \mathrm{l}]$ \\
\hline Opti-Mem Medium & 75 & 75 \\
Lipofectamine & 6 & - \\
siRNA $(10 \mu M$ stock & - & 3 \\
\hline
\end{tabular}

As the general performance of the Lipfectamine Reagent was unsatisfactory, in further approaches the HiPerFect@ Transfection Reagent (Qiagen; \#301705), specifically designed 
for the siRNAs was used. siRNAs were used at a concentration of $5 \mathrm{nM}$ in $100 \mu \mathrm{l} /$ Well mix (Detailed information of the Mix is given in Table 2.9).

Table 2.9 HiPerFect@ Transfection Mix

Component Amount $[\mu \mathrm{l}]$

\begin{tabular}{lc}
\hline Cardio Culture Medium & $100 \mu \mathrm{l}$ \\
siRNA & $0.3 \mu \mathrm{l}[5 \mathrm{nM}]$ \\
HiPerFect Transfection Reagent & $3 \mu \mathrm{l}$ \\
\hline
\end{tabular}

The mix was prepared by pipetting the siRNA in the Cardio Culture medium and vortexing, followed by addition of the transfection reagent. The final mixture was then thoroughly vortexed and incubated at RT for 10 min to allow transfection complex formation. The transfection mix was then added dropwise to the wells containing $1.9 \mathrm{ml}$ Cardio Culture Medium $(2 \mathrm{ml}$ total afterwards) and the cells incubated for 24 hours. Medium was changed to fresh Cardio Culture Medium and cells harvested 24 hours later. The knockdown was validated via Western Blot and RT-qPCR (Section 2.4 .2 - 2.4.5).

\subsubsection{Combination of siRNA transfection and ET-1 treatment}

For subsequent ET-1 treatment of the cells, a refined protocol was developed where the transfection step was repeated after 24 hours, to strengthen the silencing effect. A still active silencing effect was validated 48 hours after siRNA removal (Section 3.2.3).

After the total of 48 hours siRNA transfection, siRNA containing medium was removed and cells treated with ET-1 for 48 hours as described above. Cells were then harvested for subsequent molecular analysis.

An overview of the transfection and treatment protocols is shown in Figure 3.26.

\subsubsection{Immunohistochemistry}

For fluorescence staining of iPSC-CM, the cells were digested and counted, and 30.000 cells/Well plated in 12-Well plates with Geltrex coated $20 \mathrm{~mm}$ coverslips placed on the bottom of the well. After recovery, cells were treated with siRNA or ET-1 as described before (Section 2.3.6 - 2.3.8), with mentioned concentrations (volumes adjusted to smaller Well dimensions). After carrying out the respective treatments, medium was aspirated, cells washed with PBS and fixed with Roti®-Histofix 4\% (Roth; \#P087.1) $1 \mathrm{ml} /$ Well for $20 \mathrm{~min}$ at RT. Fixing solution was then removed and the cells washed trice with PBS (0.5-1 ml/well). After washing, the coverslips were placed in a humidified chamber and incubated with primary antibody, diluted 
in Blocking Solution (Refer to Table 2.10) at $4^{\circ} \mathrm{C}$ overnight. On the next day, the coverslips containing the cells were washed trice by dipping in PBS. Afterwards, the coverslips were incubated with secondary Antibody (conjugated to fluorescent dye) diluted in Blocking Solution for $1 \mathrm{~h}$ at $37^{\circ} \mathrm{C}$ in the humidified chamber. An overview of the used Antibodies is given in Table 2.11. Coverslips were again washed in PBS trice and mounted in ProLong ${ }^{\mathrm{TM}}$ Gold antifade reagent with DAPI (Invitrogen; \#P36935) on Superfrost®Plus Microscope Slides (Thermo Fisher; \#J1800AMNZ). The mounted coverslips were then left to dry at $4^{\circ} \mathrm{C}$ before microscopic imaging.

Table 2.10 Blocking Solution

\begin{tabular}{lc}
\hline Component & Amount [ $\mu \mathrm{l}]$ \\
\hline DPBS (Thermo Fisher; \#14190144) & $32.5 \mathrm{ml}$ \\
\hline BSA Fraction V 7.5\% (Thermo Fisher; \#15260-037) & $5 \mathrm{ml}$ \\
Triton X-100 (Thermo Fisher;\# T8787) diluted 1:10 (10\%) in PBS & $750 \mu \mathrm{l}(1 \%)$
\end{tabular}

Table 2.11 Antibodies for Immunofluorescence

\begin{tabular}{llllll}
\hline Antibody & Type & Source & Dilution & Product No. & Manufacturer \\
\hline a-Actinin & Primary & mouse & $1: 1000$ & \#A7811 & Sigma \\
anti-mouse & secondary & donkey & $1: 1000$ & \#A21202 & Thermo Fisher \\
lgG, Alexa & & & & &
\end{tabular}

Fluor 488 


\subsubsection{Fluorescence microscopy}

Fluorescence microscopy was performed using the Observer.Z1 Fluorescence Microscope (Zeiss). Images of the cells were taken at $400 \mathrm{X}$ magnification with the Plan-NEOFLUAR $40 x / 1,3$ Oil DIC objective (ZEISS) in the DAPI-channel (at $358 \mathrm{~nm}$ ) to visualize nucleus staining and the FITC-channel (at $488 \mathrm{~nm}$ excitation) to visualize the Alexa Fluor 488 secondary antibody conjugate.

\subsubsection{Cell size measurements}

The cell size of iPSC-CM was assessed by analyzing the actinin staining using ImageJ. In brief, the image was transformed into an 8-bit image and the Pixel size determined to represent a distance of $0.1614 \mu \mathrm{m}$. The Contrast was enhanced for $0.3 \%$, the Threshold set to B/W with Dark Background and Li's Minimum Entropy thresholding algorithm (Landini 2017) applied. White spots in the cell area were filled with the "Fill Holes" command. If this led to fusing of two separate cells, the areas were separated manually with the brush tool. The black areas representing cells were then selected with the "wand-tool". The measured area could be read from the ROI-Manager and exported to Excel. Calculations were performed in Excel and statistical analysis and visualization in GraphPad Prism Software (v. 8.4.2). 


\subsection{Molecular analysis}

\subsubsection{Protein extraction}

Up to $30 \mathrm{mg}$ of snap frozen mouse- or human LV-tissue or snap frozen iPSC-CM cell pellets were homogenized in ice-cold Pierce RIPA-buffer (Thermo Fischer; \#89900) supplemented with protease inhibitor (Roche; \#04693159001) and phosphatase inhibitor (Roche; \#04906845001) (one tablet each per $10 \mathrm{ml}$ RIPA-buffer) via bead disruption. For that, $5 \mathrm{~mm}$ stainless steel beads (Qiagen; \#69989) were precooled on dry ice for $15 \mathrm{~min}$ and then added to the ice-cooled tube containing tissue or cells to be lysed. Protease-/Phosphatase-inhibitor supplemented RIPA buffer was added and the samples homogenized on the Tissue Lyser LT (Qiagen; \#85600) for $5 \mathrm{~min}$ at $50 \mathrm{~Hz}$. The samples were further placed on an overhead rotor at $4^{\circ} \mathrm{C}$ for at least $1 \mathrm{~h}$ before centrifugation at $16.000 \times \mathrm{g}$ for $15 \mathrm{~min}$ at $4^{\circ} \mathrm{C}$. The supernatant was transferred to fresh $1.5 \mathrm{ml}$ Eppendorf tubes and protein concentration determined with the Pierce BCA Protein Assay Kit (Thermo Fischer; \#23225) at a 1:10 dilution at $562 \mathrm{~nm}$ absorbance in a 96 -well plate photometer (Biotek). Protein lysates were placed at $-80^{\circ} \mathrm{C}$ for long term storage.

\subsubsection{Western blotting}

For protein level analysis, SDS-PAGE Western blotting was performed. $20 \mu \mathrm{g}$ protein was mixed with 4x Laemmli Sample Buffer (Biorad; \#1610747) supplemented with 0.2M Dithiothreitol (DTT) as a reduction agent to a final Buffer concentration of $1 \mathrm{X}$. The samples were denatured for $5 \mathrm{~min}$ at $95^{\circ} \mathrm{C}$ in a T100 Thermal Cycler (Biorad; \#1861096) and then directly placed on ice until loading onto gels. Proteins were separated on 4-20\% TGX-Stainfree gels (Biorad; \#4568096) by running a constant Voltage of $200 \mathrm{~V}$ for $35 \mathrm{~min}$. The stain-free gels were activated for $1 \mathrm{~min}$ with UV light for later stain-free imaging of the membrane. Subsequently, proteins were transferred onto a $0.2 \mu \mathrm{m}$ Nitrocellulose Membrane (Biorad; \#1620214) with the Trans-Blot Turbo Transfer System (Biorad; \#1704150) at a constant current of $2.5 \mathrm{~A}$ for $5 \mathrm{~min}$ (one membrane per transfer chamber) or $7 \mathrm{~min}$ ( 2 gels per transfer chamber). Afterwards, the transfer efficiency was measured by imaging the membrane with the Stain-Free imaging program of the ChemiDoc XRS+ System (Biorad; \#1708265).

Membranes were then blocked for 1 hour at RT in 5\% milk (Roth; \#T45.3) dissolved in TBS-T (20 mM Tris-base, $50 \mathrm{mM} \mathrm{NaCl}, 0.1 \%$ Tween-20, pH 7.5). After blocking, the blots were incubated with primary Antibody (at different concentrations, Table 2.12) diluted in 1\% milk in TBS-T overnight at $4^{\circ} \mathrm{C}$ in $50 \mathrm{~mL}$ Falcon tubes on a roller to guarantee evenly incubation. On 
the next day, the membranes were washed three times for 5 min each in TBS-T and were then incubated for 1 hour with horseradish peroxidase (HRP) conjugated secondary antibodies (Table 2.12) diluted in 1\% milk in TBS-T at RT. Afterwards, the blots were again washed three times for $5 \mathrm{~min}$ and were then incubated in Clarity Max TM Western ECL Substrate (Biorad; \#1705062) for $5 \mathrm{~min}$. Chemiluminescence bands were detected using the ChemiDoc XRS+ Imager. Protein bands were then quantified with the Image Lab Software (Version 6.0.1; Biorad) with background subtraction and protein of interest (POI) bands were normalized to either housekeeping protein bands (GAPDH) or total protein (from the stain-free image). Band intensities were calculated in Microsoft Excel 2016 and visualized in GraphPad Prism Software (v. 8.4.2). An unpaired t-test or two-way ANOVA was performed depending on group assignment to determine the statistical significance of protein level changes. Western blots were performed with at least two technical duplicates. 
Table 2.12 Antibodies for Western blotting

\begin{tabular}{|c|c|c|c|c|c|}
\hline Antibody & Type & Source & Dilution & Product No. & Manufacturer \\
\hline \multicolumn{6}{|l|}{$\begin{array}{l}\text { Primary } \\
\text { Antibodies }\end{array}$} \\
\hline $\begin{array}{l}\text { FTO (5H-2H10) } \\
\text { (mouse and } \\
\text { human) }\end{array}$ & Monoclonal & Mouse & $1: 1000$ & \#NBP2-29512 & novusbio \\
\hline $\begin{array}{l}\text { METTL3 } \\
\text { (mouse and } \\
\text { human) }\end{array}$ & Polyclonal & Rabbit & $1: 1000$ & \#15073-1-AP & proteintech \\
\hline $\begin{array}{l}\text { METTL14 } \\
\text { (mouse and } \\
\text { human) }\end{array}$ & Polyclonal & Rabbit & $1: 1000$ & \#HPA038002 & Sigma \\
\hline $\begin{array}{l}\text { CALM1 } \\
\text { (human) }\end{array}$ & Polyclonal & Rabbit & $1: 1000$ & \#LS-C331329 & LSBio \\
\hline $\begin{array}{l}\text { CALM1 } \\
\text { (mouse) }\end{array}$ & Monoclonal & Rabbit & $1: 1000$ & \#ab45689 & abcam \\
\hline ANP (human) & Polyclonal & Rabbit & $1: 1000$ & \#ab126149 & abcam \\
\hline GAPDH & Monoclonal & Mouse & $1: 1000$ & MAB374 & Merck \\
\hline $\begin{array}{l}\text { Secondary } \\
\text { Antibodies }\end{array}$ & & & & & \\
\hline Anti-Mouse & & Goat & $1: 10.000$ & $7074 S$ & Cell Signaling \\
\hline $\begin{array}{l}\text { lgG, HRP- } \\
\text { linked }\end{array}$ & & & & & Technology \\
\hline Anti-Rabbit & & Sheep & $1: 10.000$ & NA931V & GE Healthcare \\
\hline $\begin{array}{l}\text { IgG, } \\
\text { linked }\end{array}$ & & & & & \\
\hline
\end{tabular}




\subsubsection{RNA isolation}

RNA isolation was carried out using the RNEasy Fibrous Tissue Mini Kit (Qiagen; \#74704) following the manufacturers instruction. For subsequent cDNA synthesis and RT-qPCR analysis the protocol is described briefly.

Up to $30 \mathrm{mg} \mathrm{LV}$-tissue or up to $30 \mu \mathrm{l}$ cell pellets were placed in $2.0 \mathrm{ml}$ RNase and DNase free Eppendorf tubes containing $7 \mathrm{~mm}$ stainless steel beads (Qiagen; \# 69990) and $300 \mu \mathrm{l}$ RLT buffer supplemented with $40 \mathrm{mM}$ DTT. The samples were homogenized using the Tissue Lyser LT (Qiagen) at $50 \mathrm{~Hz}$ for $5 \mathrm{~min}$. After homogenization, $590 \mu \mathrm{l}$ water and $10 \mu \mathrm{l}$ Proteinase $\mathrm{K}$ were added and samples incubated at $55^{\circ} \mathrm{C}$ for $10 \mathrm{~min}$ in a heating block. Afterwards, samples were centrifuged for $3 \mathrm{~min}$ at $10.000 \times \mathrm{g}$ and supernatant transferred to fresh $1.5 \mathrm{ml}$ RNase and DNase free Eppendorf tubes. A 0.5 volume of $100 \%$ ethanol (EtOH) was added and gently mixed by pipetting up and down several times. The mixture was then loaded onto a spin column and precipitated RNA bound to the silica-membrane by centrifugation for $15 \mathrm{sec}$ at $10.000 \mathrm{xg}$. The column was washed with $350 \mu \mathrm{l}$ RW1 buffer by centrifugation of $15 \mathrm{sec}$ at $10.000 \mathrm{xg}$. The column was then incubated with $10 \mu \mathrm{l}$ DNase I diluted in $70 \mu \mathrm{l}$ RDD buffer for 10 min at RT, and washed again with $350 \mu \mathrm{l}$ RW1 buffer spun at $10.000 \times \mathrm{g}$ for $15 \mathrm{sec}$. Afterwards, the column was washed with $500 \mu \mathrm{l}$ RPM buffer, supplemented with $100 \% \mathrm{EtOH}$, twice, with 15 sec centrifugation at $10.000 \times \mathrm{g}$ in the first, and $1 \mathrm{~min}$ at $10.000 \mathrm{xg}$ centrifugation in the second step. Finally, the column was dried by centrifugation at $13.000 \times \mathrm{g}$ for $1 \mathrm{~min}$. For elution of RNA the column was incubated with 15-30 $\mu$ l elution buffer, depending on desired RNAconcentration, for $1 \mathrm{~min}$ followed by centrifugation at $10.000 \mathrm{xg}$ for $1 \mathrm{~min}$. RNA was directly placed on ice. 


\subsection{4 cDNA synthesis}

For later RT-qPCR experiments, complementary DNA (cDNA) was reversely transcribed from total RNA using the iScriptTM cDNA Synthesis Kit (Biorad; \#1708891) following the manufacturers instruction. When possible, depending on RNA yield, $500 \mathrm{ng}$ of total RNA was used to synthesize cDNA in a $20 \mu \mathrm{l}$ reaction mix. The reaction mix and reaction protocol are given in Table 2.13 and Table 2.14.

Table 2.13 RT-Mix

\begin{tabular}{lc}
\hline Component & Volume $[\mu \mathrm{l}]$ \\
& \\
\hline 5x iScript Reaction Mix & 4 \\
iScript Reverse & 1 \\
Transcriptase & \\
Nuclease free $\mathrm{H}_{2} \mathrm{O}$ & fill to $20 \mu \mathrm{l}$ \\
RNA template (500ng) & variable \\
Total volume & 20 \\
\hline
\end{tabular}

Table 2.14 RT Protocol

\begin{tabular}{lrc}
\hline Step & $\begin{array}{r}\text { Time } \\
{[\mathrm{min}]}\end{array}$ & \multicolumn{2}{l}{$\begin{array}{l}\text { Temperature } \\
{\left[{ }^{\circ} \mathbf{C}\right]}\end{array}$} \\
\hline Priming & 5 & 25 \\
Reverse & 20 & 46 \\
$\begin{array}{lc}\text { Transcription } \\
\text { RT }\end{array}$ & 1 & 95 \\
inactivation & & \\
\hline
\end{tabular}

The final cDNA was diluted $1: 2$ by adding $20 \mu$ of Nuclease-free water leading to a cDNA concentration of $12.5 \mathrm{ng} / \mu \mathrm{l}$.

\subsubsection{RT-qPCR for gene expression}

To assess gene expression levels, reverse transcription quantitative real-time PCR (RT-qPCR) was used. For analysis of FTO in mouse and human tissue, a SYBR Green based approach was used, with the method briefly described below.

A reaction mix was setup by diluting the SsoAdvanced Universal SYBR Green Supermix (Biorad; \#1725272) in Nuclease-free water and forward and reverse primers were added at a concentration of $10 \mathrm{pmol} / \mu \mathrm{l}$. The mix was transferred to a 96 -well RT-qPCR plate (Biorad; HSP9601) and $12.5 \mathrm{ng}$ cDNA per well added to the mixture. water instead of cDNA served as a no-template control. The plates were sealed with adhesive film (Biorad; \#MSB1001) and centrifuged for $1 \mathrm{~min}$ at $1000 \mathrm{x}$ g before measuring in the CFX Connect Real Time PCR System (Biorad). The reaction mix and the RT-qPCR protocol are given in Table 2.15 and Table 2.16, primer names and sequences in Table 2.17.

RNA levels were normalized to $R n 18 s$ (s18) as housekeeping gene and expression level changes calculated with Excel 2016 using the ddCT method (Livak and Schmittgen 2001). Unpaired t-test or two-way ANOVA, depending on group assignment were performed and visualized with GraphPad Prism (v8.4.2). 
Table 2.15 RT-qPCR Reaction Mix

\begin{tabular}{ll}
\hline Reagent & Volume $(\mu \mathrm{l})$ \\
\hline nucleaseFree $\mathrm{H}_{2} \mathrm{O}$ & 7.8 \\
For. Primer $(10 \mathrm{pmol} / \mu)$ & 0.6 \\
Rev.Primer $(10 \mathrm{pmol} / \mu)$ & 0.6 \\
Sso Advanced SYBR & 10 \\
Green Mix & \\
cDNA (12.5ng) & 1 \\
\hline
\end{tabular}

Table 2.16 RT-qPCR Protocol

\begin{tabular}{lll}
\hline Step & Temp. $\left({ }^{\circ} \mathbf{C}\right)$ & Time \\
\hline Denaturation & 95 & $3 \mathrm{~min}$ \\
Amplification & 95 & $10 \mathrm{sec}$ \\
$(x 45)$ & \\
$\begin{array}{l}\text { Elongation } \\
(x 45)\end{array}$ & 60 & $30 \mathrm{sec}$ \\
Meltcurve & $55-95$ & $6 \mathrm{sec} / \mathrm{step}$ \\
$(x 81)$ & & \\
\hline
\end{tabular}

Table 2.17 RT-qPCR Primers

\begin{tabular}{ll}
\hline Primer & Sequence \\
\hline Fto_qPCR_F (mouse) & ACAGCCTCGGTTTAGTTCCA \\
Fto_qPCR_R (mouse) & TGCCTTGAAACCAGAACTGC \\
mRn18sFW-1 (mouse) & CATGCATGTCTAAGTACGCAC \\
mRn18sRV-1 (mouse) & GTCGCCATGTATTAGCTCTAG \\
FTOhuman_qPCR_F2 & GGAGGGTGTGACAAATGCTG \\
FTOhuman_qPCR_R2 & GCAGGTAATGTT?GGGCAAT \\
S18 Human F & ACCCGTTGAACCCCATTCGTGA \\
S 18 Human R & GCCTCACTAAACCATCCAATCGG \\
\hline
\end{tabular}




\subsubsection{RT-qPCR verification of differentially methylated transcripts}

For verification of the differentially methylated transcripts, a TagMan-probe approach was used. Briefly, LightCycler 480 probes master mix (Roche; \#04707494001) was used and supplemented with forward and reverse primer, as well as FAM-labeled probes from the Universal Probe Library. Primer and Probe specifications are given in Table 2.18. The ratios between IP and Input fractions of control (Sham or NF) samples were set to 1 and RNA level changes in diseased tissues (TAC or HF) calculated accordingly.

Table 2.18 Universal Probe Library Primer and Probes

\begin{tabular}{ll}
\hline Primer/Probe & Sequence/Library Code \\
\hline CALM1 forward (human) & TACCACGAACCCCTCAGC \\
CALM1 reverse (human) & GACCAAATTTACATTCGTTGTTCA \\
CALM1 FAM Probe & Universal Probe Library \# 48 \\
Calm1 forward (mouse) & GCTGCAGGATATGATCAACG \\
Calm1 reverse (mouse) & AGAACTCTGGGAAGTCAATGGT \\
Calm1 FAM Probe & Universal Probe Library \# 89 \\
\hline
\end{tabular}

\subsection{7 m6A methylation assay}

For analysis of the global methylation level, up to 300 ng rRNA depleted RNA was analyzed with the m6A RNA Methylation Assay Kit (abcam; \#ab185912) according to the manufacturer's instructions. m6A level detection was performed by Dr. Tea Berulava (German Center for Neurdegenerative Diseases). 


\subsection{Methylation analysis-workflow}

\subsubsection{Trizol RNA extraction}

Total RNA for subsequent MeRIP and NGS analysis was isolated with a Trizol based extraction method. Briefly, LV tissue from mouse or human was homogenized in $1 \mathrm{ml}$ Trizol Reagent (Thermo Fischer; \#15596018) for $7 \mathrm{~min}$ on the Omni Bead Ruptor at $2.6 \mathrm{~m} / \mathrm{s}$ frequency. The homogenized samples were incubated $5 \mathrm{~min}$ at RT and $200 \mu \mathrm{l}$ chloroform added. Samples were vortexed and incubated $15 \mathrm{~min}$ at RT followed by a $15 \mathrm{~min}$ centrifugation step at 14.000 $x \mathrm{~g}$. The upper phase, containing RNA, was transferred to fresh tubes and $1 \mu$ glycogen blue and $500 \mu$ isopropanol added. The mix was inverted several times to ensure thorough mixing. Samples were then frozen at $-20^{\circ} \mathrm{C}$ overnight and centrifuged the next day for 20 min at 14.000 $\mathrm{xg}$ at $4^{\circ} \mathrm{C}$. The supernatant was carefully removed without touching the pellet. $1 \mathrm{ml}$ of $75 \%$ $\mathrm{EtOH}$ was added to the pellet and centrifuged for $10 \mathrm{~min}$ at $14.000 \times \mathrm{g}$ and $4^{\circ} \mathrm{C}$ to wash the pellet. The supernatant was removed, the pellet air-dried at RT for 10-15 min and finally resuspended in $45 \mu$ l water.

\subsubsection{DNase I digestion}

After resuspension of the RNA, an in-solution DNase I digest was performed using the DNase I set (Zymoresearch; \#E1010). Briefly, $5 \mu$ I DNase I and $5 \mu$ I DNA Digestion Buffer was added to the eluted RNA and filled up with water to a total amount of $100 \mu \mathrm{l}$. The mix was then incubated at RT for 15 min and the RNA was cleaned up as described in Section 2.5.3.

\subsubsection{RNA cleanup}

To clean the RNA from the DNAse I treatment for further processing, the RNA Clean \& Concentrator $-5^{\mathrm{TM}}$ Kit (Zymo Research; \#R1013) was used following the manufacturer's instructions. Briefly, 2 vol. of RNA Binding Buffer were added to each DNase I treated sample and mixed. An equal vol. of $100 \% \mathrm{EtOH}$ was added and the mixture transferred to a ZymoSpinTM IC Column in a collection tube. RNA was bound to the column by centrifugation for 30 sec at $13.000 \times$ g. $400 \mu$ l RNA Prep Buffer was added to the column and centrifuged likewise, followed by two washing steps with RNA wash buffer, using $700 \mu \mathrm{l}$ in the first step and centrifugation for 30 seconds at $13.000 \times \mathrm{g}$ and $400 \mu \mathrm{l}$ RNA Wash Buffer in the second step, spinning for 2 min to ensure complete buffer removal. The RNA was finally eluted in $15 \mu \mathrm{l}$ DNase/RNase-Free Water with centrifugation at $13.000 \times \mathrm{g}$ for $30 \mathrm{sec}$ and immediately used or placed at $-80^{\circ} \mathrm{C}$ for storage. 


\subsubsection{RNA separation in small and large fractions}

The same RNA Clean \& Concentrator Kit was used for separation of RNAs into fractions of small RNAs (17-200nt length) and large RNAs (>200nt length) before subsequent MeRIP and NGS. This allows for later analysis of for example miRNAs, which are otherwise lost due to poor binding to the column. [Note: miRNA related experiments are not part of this thesis and just mentioned here for completeness of the Methods.] The separation occurs due to the use of different concentrations of $\mathrm{EtOH}$ in the procedure, as binding of small RNAs occurs at higher $\mathrm{EtOH}$ concentrations. Briefly, this time RNA Binding buffer is supplemented with an equal volume of $100 \% \mathrm{EtOH} .2$ vol. of this mixture are added to the RNA sample. The samples were then transferred to the spin-column and centrifuged for $30 \mathrm{sec}$ at $10.000 \mathrm{xg}$. RNAs with a size bigger than $200 \mathrm{nt}$ are retained in the column, whereas smaller RNAs are in the flow-through. Therefore, the flow-through is spared and 1 vol. $100 \% \mathrm{EtOH}$ is added to this fraction. The mixture is then placed on a new spin-column and centrifuged for $30 \mathrm{sec}$ at $13.000 \mathrm{x} \mathrm{g}$ which binds the small RNAs to the column. In succeeding steps, all columns (the ones with large fractions bound and the ones with small fractions bound) are treated in the same way as already described (Section 2.5.3: $400 \mu$ l RNA Prep Buffer 30 sec centrifugation, $700 \mu$ RNA Wash Buffer 30 sec centrifugation, $400 \mu$ RNA Wash buffer 2 min centrifugation, all at 13.000 $\mathrm{x} \mathrm{g}$ ) and the RNA fractions eluted in $15 \mu \mathrm{l}$ water. Long fraction samples were directly used or placed at $-80^{\circ} \mathrm{C}$ for storage.

\subsubsection{RNA concentration measurement}

The eluted RNA concentration was determined using the Nanodrop 2000 (Thermo Fischer) Photometer measuring the absorbance at $260 \mathrm{~nm}$.

\subsection{6 rRNA depletion}

To remove ribosomal RNAs ( $r R N A$ ), which can make up to $80 \%$ of the total RNA (Lodish 2000) and can be methylated itself (Dominissini et al. 2012), which would interfere with further analysis, the NEBNext@ rRNA Depletion Kit (New England Biolabs Inc.;\# E6310) was used following the manufacturer's instructions. Up to $25 \mu \mathrm{g}$ of the long RNA fraction was subjected to rRNA depletion by adding $3 \mu \mathrm{l}$ RNA/Probe master mix $(1 \mu \mathrm{l}$ NEBNext rRNA Depletion Solution $+2 \mu \mathrm{l}$ Probe Hybridization Buffer) to the RNA (adjusted to $12 \mu \mathrm{l}$ with water). The samples were mixed by pipetting up and down 10 times and then placed in a heating block at $95^{\circ} \mathrm{C}$ initial temperature. Over $20 \mathrm{~min}$, the temperature was continuously decreased to $22^{\circ} \mathrm{C}$ to form DNA-RNA hybrids. The hybrids were digested by RNase $\mathrm{H}$ in the next step. For this, 5 
$\mu$ l of RNase $\mathrm{H}$ mix ( $2 \mu \mathrm{l}$ RNase $\mathrm{H}, 2 \mu \mathrm{l}$ RNase $\mathrm{H}$ Reaction Buffer, $1 \mu$ l Nuclease-free water) was added to the samples and mixed by pipetting up and down 10 times. After a brief centrifugation, samples were placed in a heating block at $37^{\circ} \mathrm{C}$ for 30 min for the RNase $\mathrm{H}$ digest. Afterwards, RNase $\mathrm{H}$ was inactivated by DNase I Digestion. For this, $30 \mu \mathrm{l}$ DNase I Digestion Master Mix (5 $\mu$ l DNase I Reaction Buffer, 2.5 $\mu$ l DNase I, $22.5 \mu$ l Nuclease-free water) was added to the samples and incubated at $37^{\circ} \mathrm{C}$ for $30 \mathrm{~min}$ in a heating block.

Samples were then cleaned with the Zymoresearch RNA Clean\&Concentrator Kit as described before (Section 2.5.3)

\subsubsection{RNA integrity measurement}

A successful rRNA depletion and good RNA integrity was assessed with help of the 2100 Bioanalyzer (Agilent; \#G2939BA) using the Agilent RNA 6000 Pico Kit (Agilent; \#5067-1513) following the manufacturer's instructions.

\subsubsection{Fragmentation to $80 \mathrm{nt}$}

rRNA depleted RNA was subjected to RNA fragmentation to obtain $\sim 80$ nt long fragments. This is necessary because of the size limitation of nucleotides the Illumina Platform can Sequence in one piece. Fragmentation was performed using RNA Fragmentation Reagents (Thermo Fischer; \# AM8740). The RNA was brought to a volume of $18 \mu$ with Nuclease-free water and $2 \mu \mathrm{l}$ 10X Fragmentation Buffer was added. The mix was vortexed, centrifuged briefly and incubated for $12 \mathrm{~min}$ at $70^{\circ} \mathrm{C}$ in a heating block. Afterwards, the fragmentation was stopped by addition of $2 \mu \mathrm{l}$ Stop Solution and samples cleaned again as described before (Section 2.5.3). To check if fragmentation was successful and the desired nt-size was acquired, samples were again measured with the Bioanalyzer setup as described before (Section 2.5.7).

\subsubsection{MeRIP}

To obtain m6A methylated RNA, magnetic bead-based immunoprecipitation was carried out. First, $30 \mu \mathrm{l}$ Protein A/G magnetic beads (Thermo Fischer; \#88802) were washed twice with $500 \mu \mathrm{l} 1 \mathrm{X}$ IP Buffer (Table 2.19) and then resuspended in $500 \mu \mathrm{l} 1 \mathrm{X}$ IP Buffer. $5 \mu \mathrm{g}$ of m6A antibody (Sysy; \#202003) was added and incubated on an overhead rotor at $4^{\circ} \mathrm{C} O N$. The next day, the beads were separated on a magnetic stand and washed twice with $500 \mu$ IX IP Buffer and finally resuspended in $200 \mu \mathrm{l}$ 1X IP Buffer. $7-8 \%$ of the rRNA depleted, fragmented RNA was spared as Input, the rest was diluted in the $200 \mu$ IX IP Buffer supplemented with $5 \mu$ l (20 $\mathrm{U} / \mu \mathrm{l}$ ) Superase.IN RNase Inhibitor (Thermo Fisher; \#AM2694). The samples were then added 
to the Bead-Buffer mixture and placed on an overhead rotor for 2 hours at $4^{\circ} \mathrm{C}$. Afterwards, the beads were separated on a magnetic stand, the supernatant discarded, and the beads washed twice in $500 \mu \mathrm{l} 1 \mathrm{X}$ IP Buffer. To detach the m6A antibody with the bound methylated RNA fragments from the beads, $6.7 \mathrm{mM}$ N6-methyladenosine (Sigma; \#M2780) in 1X IP Buffer was added. The beads were separated on a magnetic stand and the supernatant containing the methylated RNA fractions transferred to new DNase/RNase-free tubes. The elution step was repeated to end up with $300 \mu$ l eluted RNA in total. The RNA was finally cleaned again with the Zymoresearch Clean \& Concentrator (Section 2.5.3) and eluted in 7 $\mu$ l Nuclease-free water.

Table 2.19 5X IP Buffer

\begin{tabular}{ll}
\hline Components & Concentration \\
\hline Tris & $250 \mathrm{mM}$ \\
$\mathrm{NaCl}$ & $750 \mathrm{mM}$ \\
$\mathrm{NP}-40$ & $0.5 \%$
\end{tabular}

For 1X IP Buffer: dilute 1:5 with $\mathrm{H}_{2} \mathrm{O}$

\subsubsection{Library preparation}

cDNA libraries were prepared from immunoprecipitated (Iped) and Input samples using the TruSeq Stranded Total RNA Library Prep Kit (Illumina; \#20020596). Library Preparation was done by Susanne Burkhard, German Center for Neurodegenerative Diseases.

Iped samples and Input libraries were Seqenced on the Illumina HiSeq2000.

\subsubsection{Bioinformatical analysis of sequencing data}

Sequenced data was transformed for analysis. First, adapters were timed from the original reads, low quality reads removed, and remaining reads mapped using STAR tools to the corresponding genome of the used tissue, either mouse genome (mm10) or human genome (hg38). SAMtools was used for sorting and indexing of mapped reads and bam files were generated. These data processing was done for Input, as well as Iped fraction data of each sequenced biological sample (replicate). In mouse hypertrophy condition, 6 Sham and 6 TAC samples passed quality control and were analyzed. In mouse heart failure 5 Sham and 6 TAC samples were of sufficient quality, in human heart failure $5 \mathrm{NF}$ and $6 \mathrm{HF}$ samples.

For transcriptome analysis, differential expression analysis was performed using DESeq2 (Michael Love 2017) on the Sequencing data of Input samples of control (Sham or NF) and diseased hearts (TAC or HF). Genes whose transcript levels showed 2-fold or more change with Padj-values equal or less than 0.05 were considered as differentially expressed. 
For detection of methylation peaks the MeTPeak package (Cui et al. 2016) was used. bamfiles of Input and led samples of all replicates were submitted to the package and peaks significantly enriched in Iped samples vs. Input samples detected. Only those with false discovery rate (FDR) equal or less than 0.05 were considered real m6A-peaks. Peaks were further assigned based on their position in mRNA, in particular 5' UTR, CDS and 3' UTR.

Differential methylation analysis was performed with an in-house developed pipeline. Briefly, overlapping or unique peaks for treated and control samples were detected using BEDTools. Fold changes with joined $p$-values were calculated. Increased fold changes represent hypermethylation, decreased fold change hypomethylation. In this study, only transcripts with at least 2 -fold change $(p \leq 0.05)$ in either direction of all peaks detected for the transcript were considered in this study.

Data processing and bioinformatic analysis of sequencing data was performed by the Bioinformatic Unit of the German Center for Neurodegenerative diseases, especially Dr. Tea Berulava, Dr. Gaurav Jain and Tonatiuh Pena.

\subsubsection{Analysis of polysome-associated RNAs}

Polysome preparation was performed as previously described (Chassé et al. 2017) with LV tissue from Sham and TAC animals. The tissue was disrupted using a MICCRA D-1 homogenizer in lysis buffer $(50 \mathrm{mM}$ Tris- $\mathrm{HCl} \mathrm{pH} \mathrm{7.4,10} \mathrm{mM} \mathrm{MgCl}, 100 \mathrm{mM} \mathrm{NaCl}, 1 \%$ Triton$\mathrm{X}-100,1 \mathrm{mM}$ DTT, RNase inhibitor, $100 \mu \mathrm{g} / \mathrm{ml}$ cycloheximide). Centrifugation at $20.000 \mathrm{xg}$ for $10 \mathrm{~min}$ at $4^{\circ} \mathrm{C}$ removed cell debris and the whole cell extracts were separated on $10-50 \%$ sucrose density gradients (prepared in lysis buffer without RNase inhibitors) in an SW40Ti rotor Ultracentrifuge at $35.000 \mathrm{rpm}$ for 3 hours. Fractions containing polysome were determined by measuring absorbance at $260 \mathrm{~nm}$ of each fraction. Polysome fractions of each animal were pooled and RNAs extracted using phenol:chloroform:isoamylalcohol in a 25:24:1 ratio with subsequent $\mathrm{EtOH}$ precipitation. Precipitated RNA was resuspended in water and Sequenced as described above. Polysomal preparation was performed by Dr. Katherine E. Bohnsack, Department of Molecular Biology.

Polysomal RNAs were compared to Input samples using the DESeq2 package to detect enriched and depleted transcripts. Transcripts with at least 2-fold change and Padj $\leq 0.05$ were considered as differentially translated. 


\subsubsection{GO term analysis}

For representation and visualization of Gene ontology (GO) terms and pathways, the Cytoscape plugin ClueGO (v. 2.3.2) was used (Bindea et al. 2009). GO categories from 'Biological Processes' were analyzed. The network specificity was set to 'medium', statistical parameters were 'Bonferroni corrected $p$-value $<0.01$ ' and 'fuse GO term' option selected. The $\mathrm{GO}$ terms were extracted and plotted with $\mathrm{R}$ as heatmaps corresponding to their $p$-value to derive the top-significant terms.

\subsection{Software used for visualization and statistical analysis}

Graphs were created with Graph Pad Prism Software (v.8.4.2) or R (v. 3.6.2). Biorender (BioRender.com) was used to create Figure 1.1, Figure 1.2, Figure 3.26, Figure 3.28, Figure 4.1 and Figure 4.2. Other Figures were arranged with Inkscape (v. 1.0). Significance levels are shown in Figures when statistical tests were performed and are as follows: n.s : $p \geq 0.05,{ }^{*}=$ $p \leq 0.05,{ }^{* *}=p \leq 0.01,{ }^{* * *}=p \leq 0.001,{ }^{* * * *}=p \leq 0.0001$. 


\section{Results}

\section{1 m6A Methylation in mouse and human hearts}

\subsubsection{Basal characterization of the m6A methylation level in hearts}

RNA methylation has been extensively studied in various diseases and fields of biology, such as cancer (Chen et al. 2019; Liu et al. 2020), neurodegenerative diseases (Widagdo and Anggono 2018; Han et al. 2020) and developmental biology (Geula et al. 2015). Yet, relatively little is known about its role in cardiovascular diseases. In addition to our own publication (Berulava et al. 2020), only three studies relevant to this topic have been published so far. They were reporting on m6A methylation in cardiac infarction (Mathiyalagan et al. 2019), m6A methylation in dilated cardiomyopathies (Kmietczyk et al. 2019), and METTL3 manipulation in hypertrophic responses (Dorn et al. 2019). None of the studies have provided a description of the m6A-specific epitranscriptome of the heart.

To identify and characterize the basal state of m6A RNA methylation in the heart, healthy tissue from human and mouse hearts was analyzed.

\subsubsection{The methylation machinery is present in cardiac tissue}

The reversibility of the m6A marks is ensured by the reader and eraser proteins. The METTL3METTL14 heterodimer is the main writer-complex of m6A in mRNAs (Zaccara et al. 2019), therefore it was analyzed if those proteins are present in heart tissue of mice and humans by Western blotting (Figure 3.1). Similarly, the eraser protein was investigated as well. Since ALKBH5 was reported to be mainly present in testes (Zheng et al. 2013), FTO was the demethylase of interest in this study analyzed by Western blotting as well (Figure 3.1). For all three proteins, specific bands were detected, indicating the presence of the methylation machinery in both, mouse and human tissue and therefore m6A methylation can take place in cardiac tissues. Except for METTL3 in mouse tissue, which shows decreased protein level in TAC compared to Sham, the methylation machinery is unchanged on the protein level in diseased heart tissue compared to healthy control condition. It needs to be mentioned, that the used antibodies for METTL3 and METTL14 produced unspecific bands of unknown origin in addition to the expected, specific protein bands of interest (Appendix Figure 5.1), but that the bands of correct size were quantified. 
A
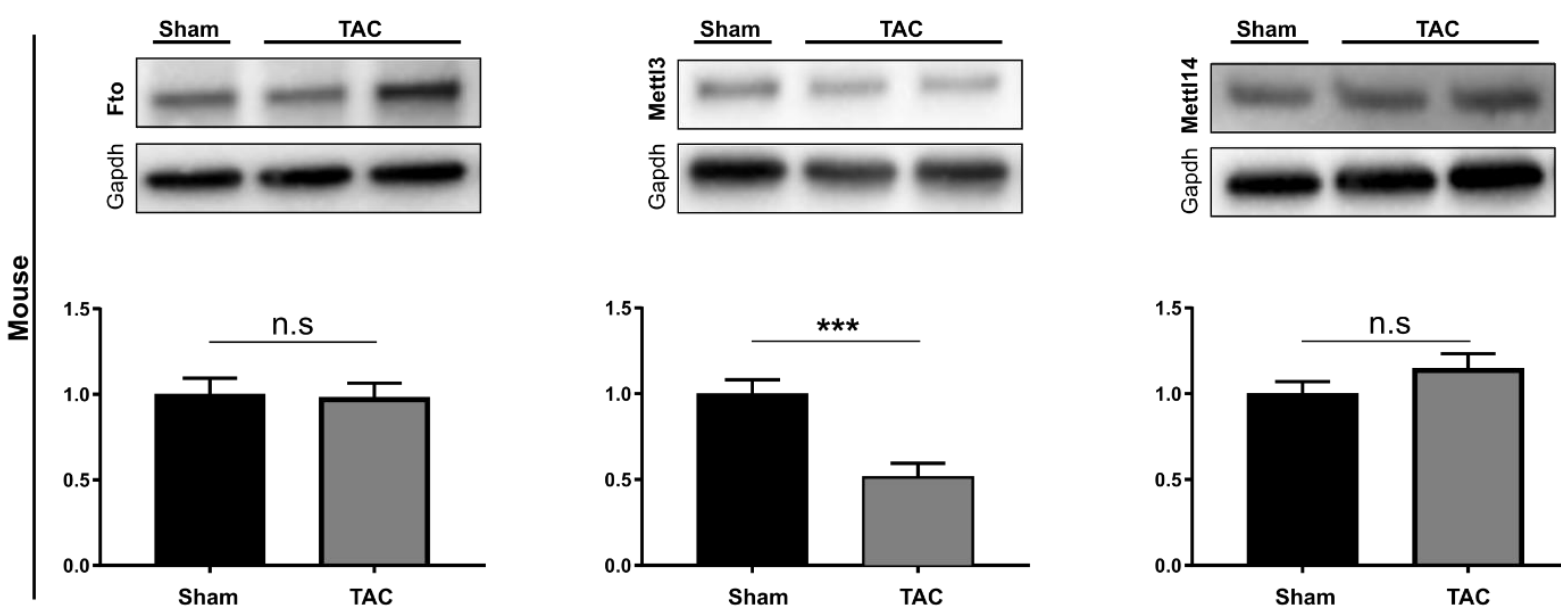

B
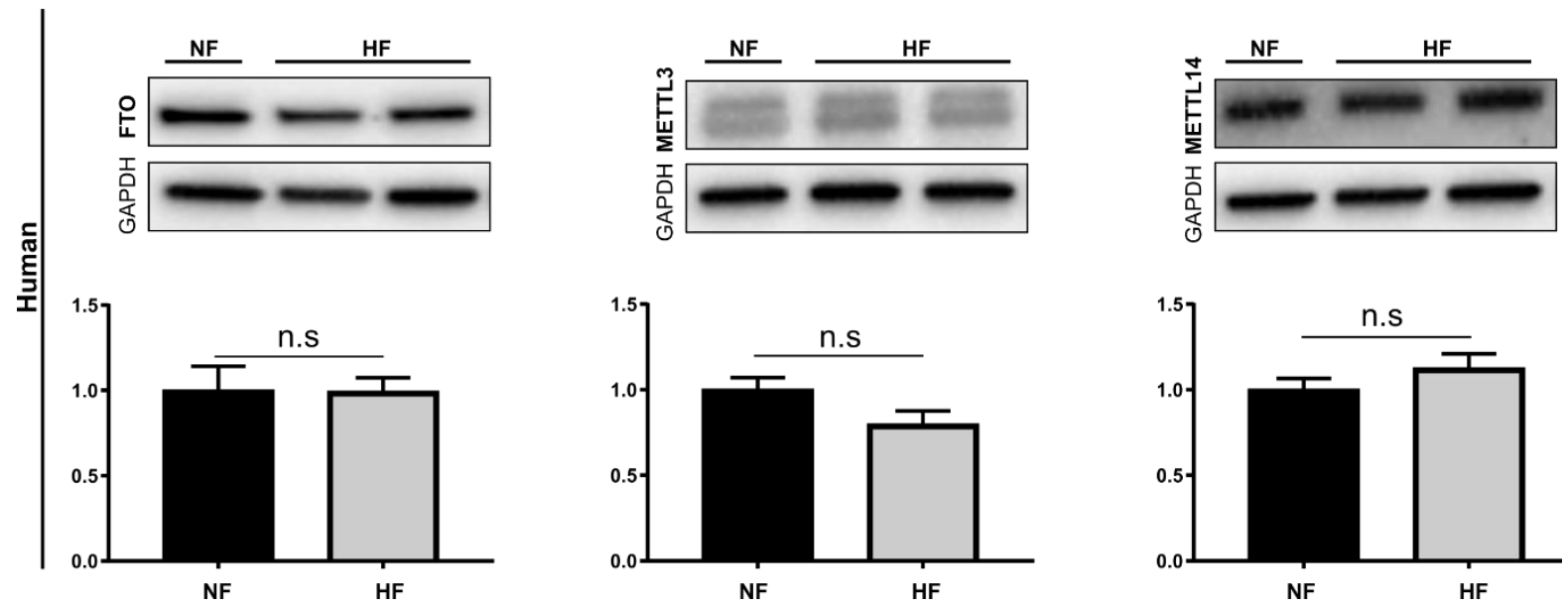

Figure 3.1 The m6A methylation machinery is present in mouse and human heart tissue (A) Key players of m6A RNA methylation, including methyltransferases METTL3 and METTL14, as well as the demethylase FTO, are present in mouse cardiac tissue as demonstrated via Western blotting. Upon TAC, FTO and METTL14 are unchanged whereas METTL3 protein level decreases. (B) Western blot analysis of the methylation machinery in human cardiac tissue demonstrates that the protein level of FTO, METTL3 and METTL14 is unchanged in failing hearts compared to NF tissue. The presence of methylation writers and erasers indicates that m6A methylation is present as a reversible mechanism in the heart. Bands of interest were normalized to GAPDH and the protein level was normalized to Sham or NF control tissue. $n=6$ for Sham, $n=12$ for TAC, $n=4$ for NF and $n=8$ for HF. t-test with Welch's correction was used to analyze protein level changes and error bars indicate SEM. Full blots are shown in the Appendix Figure 5.1.

\subsubsection{Methylation characteristics of healthy mouse and human hearts}

To assess the m6A methylation state of cardiac tissues, RNA isolated from healthy mouse ( $\mathrm{n}$ $=6)$ and healthy, non-failing human tissue $(n=5)$ was subjected to MeRIP followed by NGS. In sequenced mouse samples, $3208 \mathrm{~m} 6 \mathrm{~A}$ peaks were detected which could be mapped to 2164 transcripts (Figure 3.2 A left panel). This corresponds to $24.09 \%$ of all transcripts detected via NGS. A similar percentage of transcripts were found to be methylated in the human 
samples, wherein $1239 \mathrm{~m} 6 \mathrm{~A}$ peaks were detected, consisting of $14.6 \%$ of all detected transcripts (Figure $3.2 \mathrm{~A}$ right panel). This shows that a considerable number of transcripts carry methylation marks in the heart. A majority of these methylation marks was detected in the CDS and 3' UTR of both mouse and human tissues (CDS: $60.5 \%$; 3' UTR: $26.2 \%$ in mouse, CDS: $57.8 \%$, 3' UTR: $34.8 \%$ in human) (Figure 3.2 A). An unbiased motif search of methylated transcripts identified the consensus sequence $\mathrm{DRACH}$ as the motif mainly enriched for m6A methylation (Figure 3.2 B). This consensus sequence was previously reported to be most enriched for m6A in other tissues as well (Linder et al. 2015). The relative quantification of the distribution of m6A-marks along the mRNA transcripts shows an enrichment of methylation peaks towards the 3' UTR and the stop codon in mouse and human tissue alike (Figure 3.2 C). A similar distribution was reported in other tissues likewise (Dominissini et al. 2012; Meyer et al. 2012; Chang et al. 2017). 
A

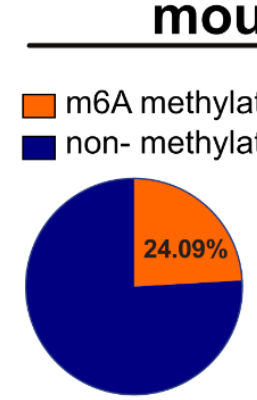

Total $\mathbf{8} 8984$

\section{m6A peaks}

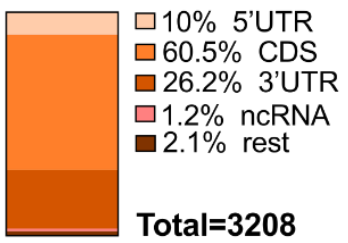

B

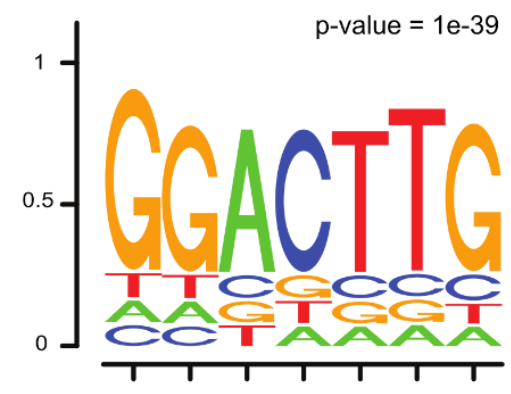

C

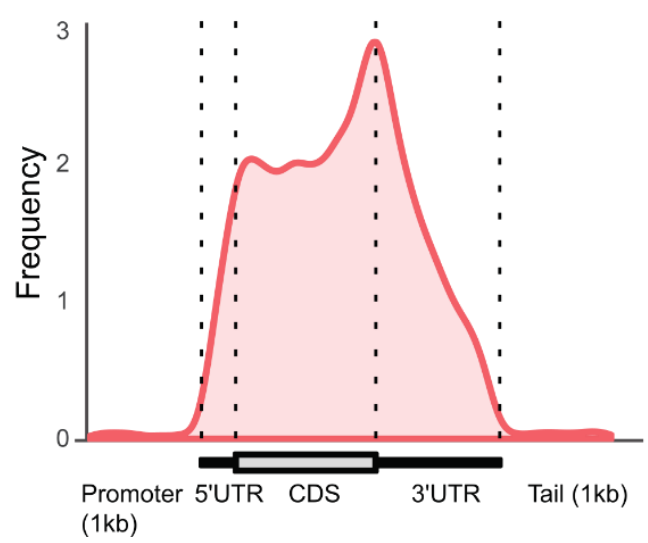

\section{human}

$\square$ m6A methylated transcripts

non- methylated transcripts
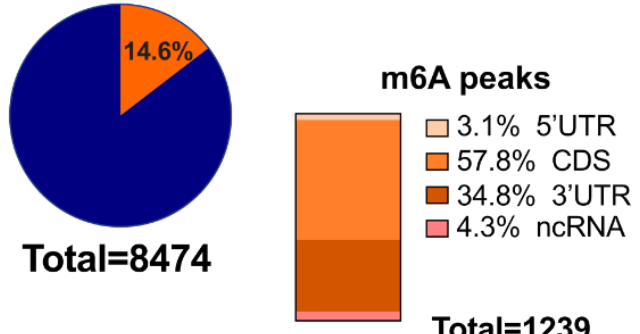

Total=1239
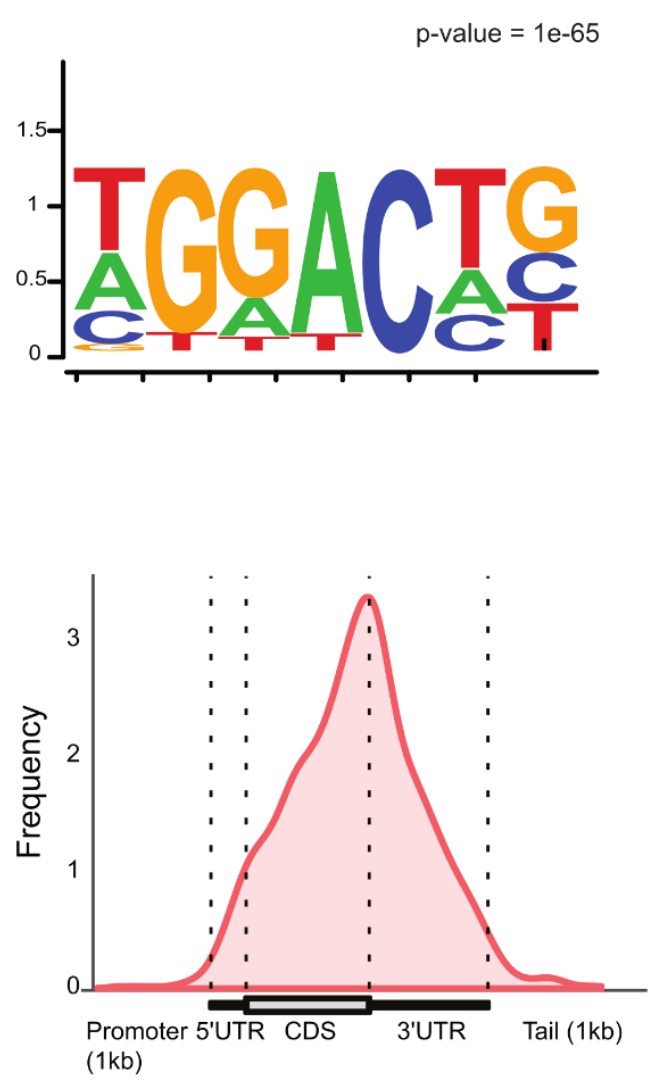

Figure 3.2 Typical methylation patterns were detected in mouse and human heart tissue (A) The pie charts show, that $24.09 \%$ of all 8964 detected transcripts carry methylation marks in mouse tissue (left panel), and $14.9 \%$ of all 8474 transcripts carry methylation marks in human tissue (right panel). Of these, most methylation marks are present in the coding sequence (CDS) and the 3' UTR in both, mouse and human tissue as indicated by the bar charts. (B) The sequence motif most enriched for m6A shows the typical DRACH sequence $(D=A, G$ or $T ; H=A, C$ or $T$ ) in mouse (left panel) and human (right panel) heart tissue. (C) The distribution of m6A marks across mRNA transcripts shows enrichment toward the transcription end site in heart tissue of both organisms. Data was obtained by sequencing of $n=5$ samples of healthy mouse and $n=5$ healthy human tissues.

Further investigation of the distribution of $\mathrm{m6A}$ marks across the transcripts (Figure 3.3) revealed, that transcripts carry m6A modifications often in the CDS and 3' UTR (544 in mouse, 82 in human) or the CDS and 5' UTR (178 in mouse, 38 in human) at the same time. Exclusive methylation in only one region was identified in mouse and human in the CDS (1099 in mouse, 
582 in human) and 3' UTR (163 in mouse, 342 in human), and a single region methylation was seen in the 5 ' UTR of human (33), whereas only a small number of transcripts are solely methylated in the 5' UTR of mice (9).

A

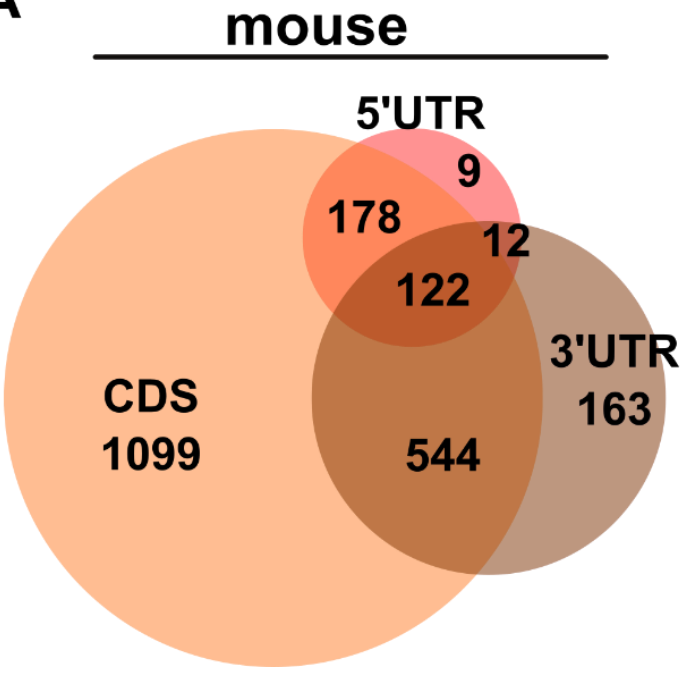

B
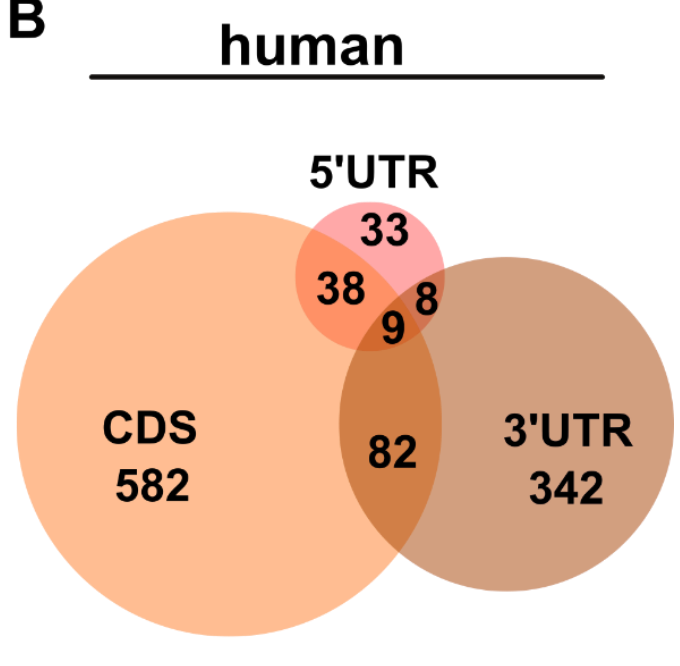

Figure 3.3 Many transcripts carry methylation marks in several regions

The Venn-diagram shows the distribution of methylation sites along RNA transcripts. In the mouse (left panel) and human (right panel) hearts, many transcripts are methylated in both, the CDS and 3' UTR or CDS and 5' UTR at the same time. Only a minority of transcripts is methylated in the 5' UTR and 3' UTR at the same time (12 in mouse and 8 in human). In both, mouse and human a majority of transcripts carry methylation marks exclusively in the CDS. In human, a considerable number of transcripts also carries methylation marks solely in the 3' UTR.

Transcripts were grouped based on the location of methylation marks and their function was investigated to identify if a pattern existed. Due to the frequent occurrence of methylation marks in the CDS and 5' UTR, as well as the exclusive methylation observed in the 3' UTR, GO analysis was performed for transcripts carrying m6A marks in the corresponding regions (Figure 3.4 A, C). It is important to notice, that from the initially identified transcripts methylated in mouse and human tissues, only those with an expression base mean above the $15 \%$ quantile for mouse and $10 \%$ quantile for human were considered for further analysis.

GO analysis revealed that transcripts with m6A-marks in both the 5' UTR and CDS mainly link to metabolic processes and mitochondrial pathways, such as 'mitochondrial electron transport', 'mitochondrial respiratory chain complex III assembly', 'mitochondrion organization', and 'mitochondrial translation' in the mouse heart (Figure 3.4 A left panel). A pattern may be seen in the human heart, where the 'mitochondrial electron transport' pathway is also among the top GO terms (Figure 3.4 C left panel). Other signal transduction pathways were also linked to the transcripts methylated in both the 5' UTR and CDS, such as 'negative regulation of intracellular signal transduction' (mouse, Figure 3.4 A left panel), and 'positive regulation of canonical Wnt 
signaling pathway' (human, Figure 3.4 C left panel). The pathway 'sarcomere organization' is present in both, mouse and human transcripts methylated in the 5' UTR and CDS (Figure 3.4 A, C).

Analysis of transcripts methylated exclusively in the 3' UTR reveals that genes involved in smooth muscle cell regulation pathways are present in the top GO terms in datasets of both mouse (Figure 3.4 A right panel) and human (Figure 3.4 C right panel). Furthermore, 3' UTR methylated transcripts code for specific metabolic processes, such as 'acyl-CoA biosynthesis', 'positive regulation of protein dephosphorylation' (mouse, Figure 3.4 A right panel), 'regulation of Rho protein signal transduction', and 'adrenergic receptor signaling pathway involved in heart process' (human, Figure 3.4 C right panel). Although one does not know what methylation of specific transcript regions results in, a different pattern of GO terms is observed for genes harboring m6A in different transcript regions. In general, methylated transcripts code for signal transduction and metabolic as well as catabolic processes.

A mild correlation between site-specific RNA methylation and RNA expression at baseline was observed (Figure 3.4 B, D). Transcripts which carry m6A marks in the 5' UTR and CDS are more abundant at the expression level, as seen by a mild positive correlation between 5' UTR and CDS methylated transcripts and expression level $(r=0.10 ; p=5.86 \mathrm{e}-07)$ in mouse (Figure 3.4 B left panel) and human ( $r=0.19 ; p=0.3 .85 e-08)$ (Figure 3.4 D left panel). The opposite was seen with transcripts methylated in the 3' UTR in mouse tissue, where a mild negative correlation between methylation and expression level was seen in mouse $(r=-0.08 ; p=0.006)$ (Figure 3.4 B right panel), indicating a lower abundancy on the expression level with increasing m6A enrichment in the 3' UTR. No such correlation was seen in human tissue $(r=-0.05 ; p=$ 0.3248) (Figure 3.4 D right panel). 
A 5'UTR and CDS

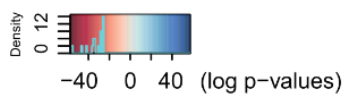

40 (log p-values)

ular protein metabolic process

mitochondrial electron transport, ubiquinol to cyt. c

negative regulation of protein complex assembly

mitochondrial respiratory chain complex III assembly

mitochondrion organization

mitochondrial translation

negative regulation of intracellular signal transduction

sarcomere organization

regulation of cellular protein catabolic process

B

regulation of cellular protein

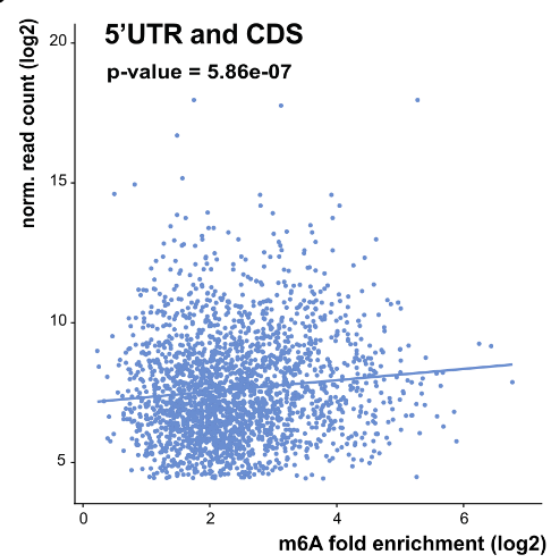

C

5'UTR and CDS

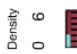

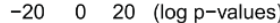

sarcomere organization

regulation of muscle filament sliding

regulation of catabolic process

ERAD pathway

positive regulation of canonical Wnt signaling pathway

protein modification by small protein conjugation or

organelle organization

mitochondrial electron transport, ubiquinol to cyt c

endothelium development

D

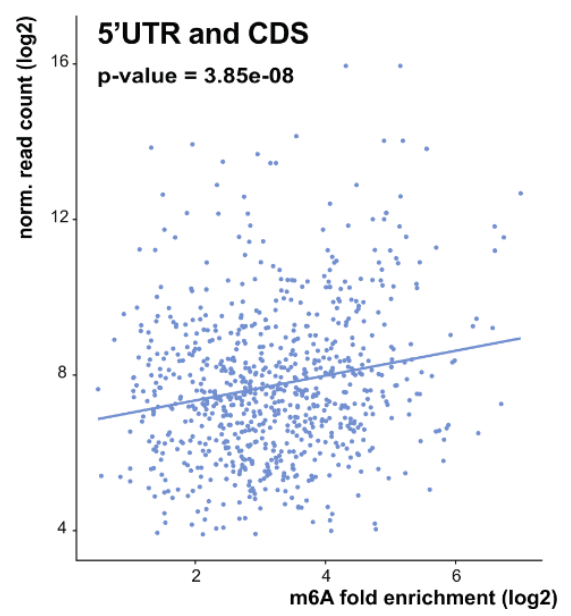

3'UTR

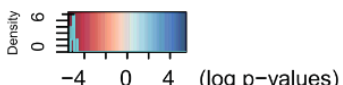

positive regulation of smooth muscle cell proliferation atrioventricular valve morphogenesis

acyl-CoA biosynthetic process

positive regulation of protein dephosphorylation

glycerolipid biosynthetic process

pos. regulation of proteasomal ubiquitin-

lysosomal transport dependent protein catabolic process

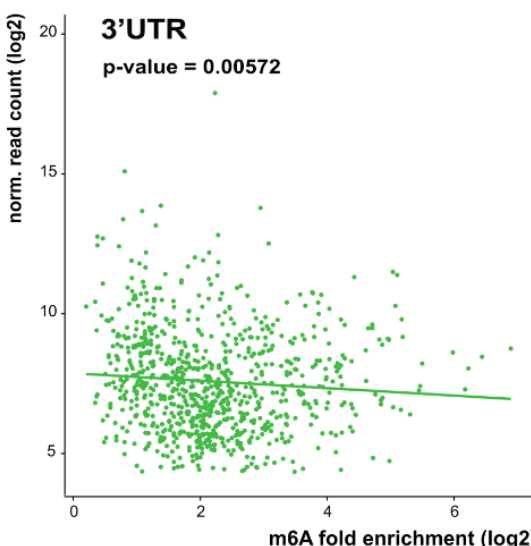

3'UTR
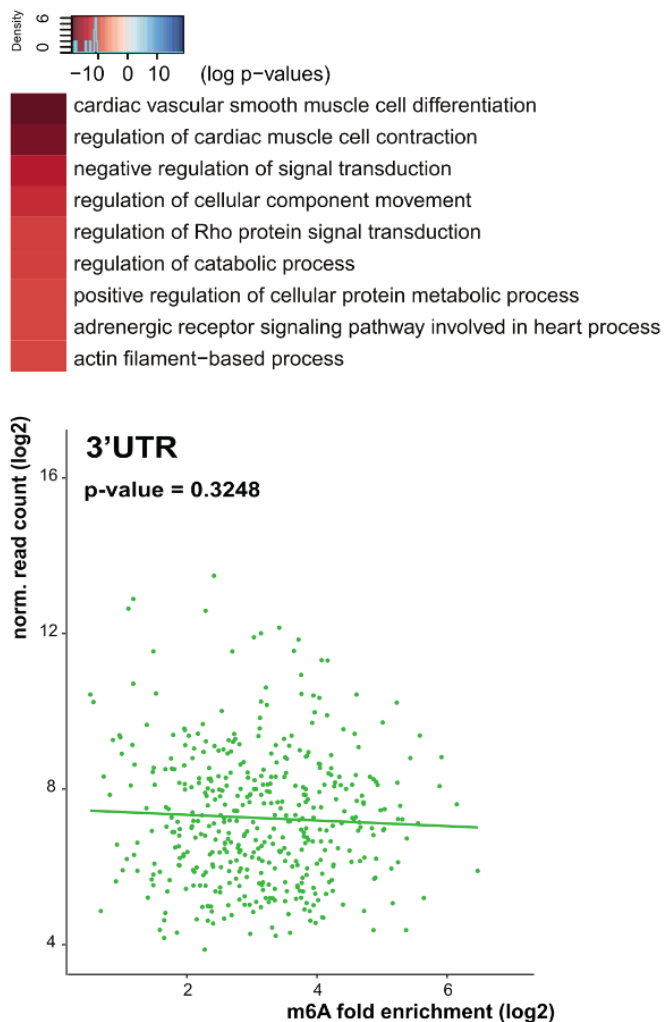

Figure 3.4 Methylation marks in different regions are associated with different biological functions and reveal a correlation with expression level

(A) The heatmap shows the most represented GO pathways of transcripts which carry methylation marks in the 5' UTR and CDS (left panel) or the 3' UTR only (right panel) in mouse. (B) A correlation analysis of transcripts methylated at specific sites, with their expression levels showing a mild positive correlation ( $r=0.10 ; p=5.86 \mathrm{e}-07$ ) of transcripts methylated in the 5' UTR and CDS, and a mild 
negative correlation $(r=-0.08 ; p=0.006)$ in 3' UTR methylated transcripts in mouse. (C) The heatmap shows the most represented GO terms of transcripts carrying m6A methylation in the 5' UTR and CDS (left panel) or 3' UTR only (right panel) in human tissue. (D) In human data, correlation analysis reveals a mild positive correlation ( $r=0.19 ; p=3.85 \mathrm{e}-08)$ between 5 ' UTR and CDS methylated transcripts and the expression level. No significant correlation is seen $(r=-0.05 ; p=0.3248)$ between 3' UTR methylated transcripts and their respective expression levels. Pearson correlation was calculated to determine the correlation coefficient $r$ and the significance $p$.

\subsubsection{Comparison of methylation in healthy mouse and human hearts demonstrates that methylated transcripts are cardio-specific}

To further compare the role of RNA methylation between healthy mouse and human hearts, transcripts from these two organisms which carry $\mathrm{m} 6 \mathrm{~A}$ methylation marks were compared (Figure 3.5). Of all the methylated transcripts, 550 were found to be present in both organisms. GO term analysis of these common transcripts demonstrated, that they are cardio-specific, as indicated by a GO cluster of 'heart development', 'circulatory system development', and 'vasculogenesis'. In line with the enriched GO pathways identified in 5' UTR and CDS methylated, and 3' UTR exclusively methylated transcripts (Figure 3.4 A, C), clusters representing metabolic processes (e.g. 'polysaccharide metabolic process') were present, as were clusters indicating a potential link with mitochondria, represented by the GO term 'energy derivation by oxidation of organic compounds'.

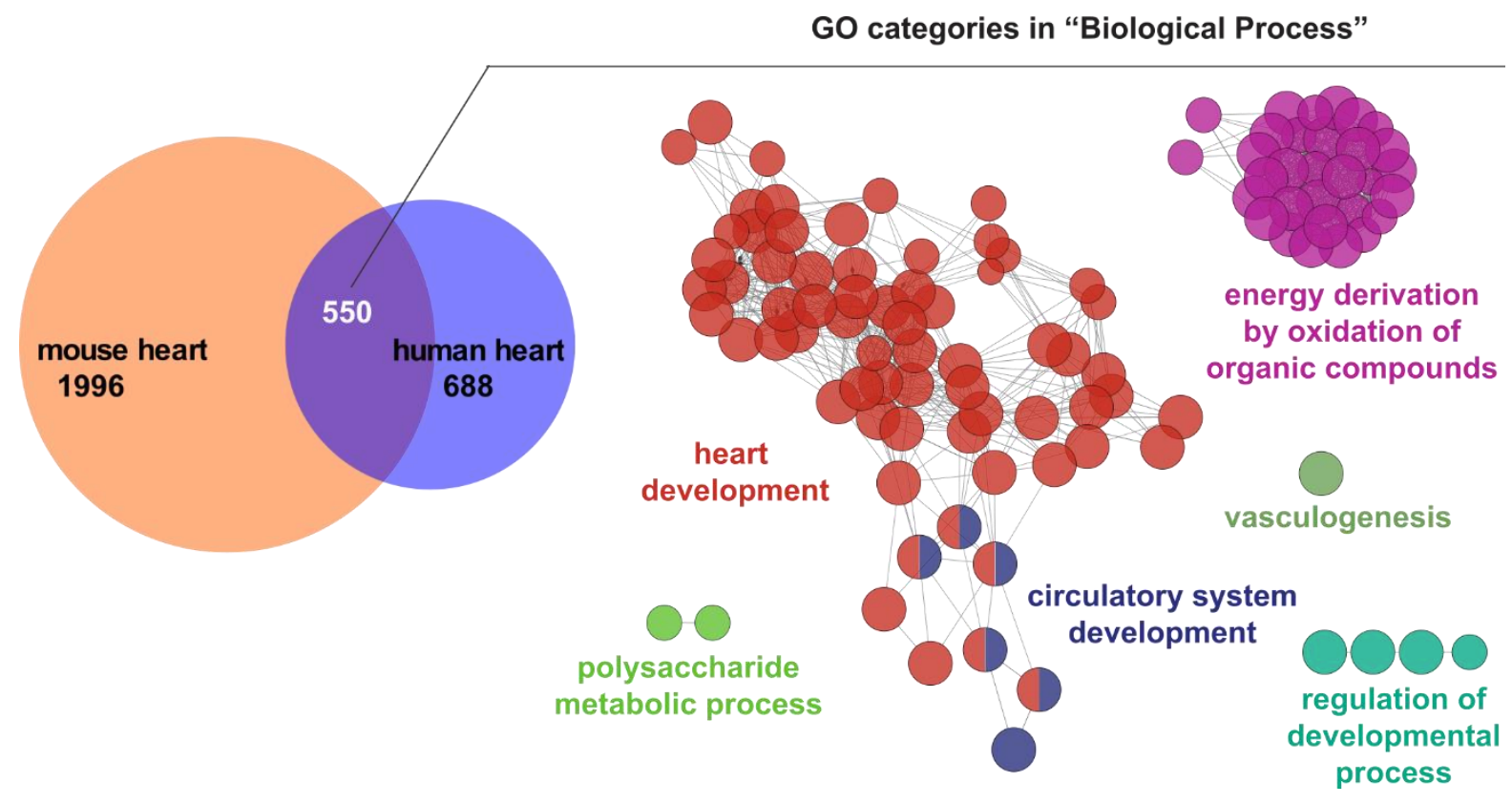

Figure 3.5 Transcripts methylated in both mouse and human tissues play a role in cardiospecific pathways

The Venn diagram represents a significant overlap of 550 genes (representation factor $7.6 ; p<2.37 \mathrm{e}-$ 21) which code for methylated transcripts in the cardiac tissue of mice and humans. A GO term analysis of these commonly methylated transcripts revealed cardiac specificity as indicated by clusters such as 'heart development', 'circulatory system development', and several metabolic and oxidative processes. 


\subsubsection{The Transcriptome changes in hypertrophy and heart failure}

To assess the effect of m6A RNA methylation in cardiac hypertrophy $(\mathrm{HT})$ and its progression to heart failure (HF), a transverse aortic constriction (TAC) model was used, where banding of the aortic branch models PO. Two time points after surgery were investigated via echocardiographic analysis to monitor the progression of the disease from compensated HT (one-week post-TAC) to HF (8 weeks post-TAC). The ejection fraction (EF) was measured to assess cardiac performance and the anterior wall thickness (AwTh) to analyze hypertrophic growth. Heart tissue obtained at both time points was analyzed for RNA expression via NGS as well as methylation changes by applying MeRIP-seq.

\subsubsection{TAC-induced cardiac hypertrophy}

One week after the TAC surgery (Figure 3.6), HT was observed in affected mice $(n=5)$, as demonstrated by a thickened left ventricular wall (Figure $3.6 \mathrm{~A}$ left), represented by an increased AwTh by $0.37 \mathrm{~mm}$ in TAC animals compared to Sham $(p=0.0001)$. The preserved EF in TAC animals (Sham: $59 \pm 5.0 \%$, TAC: $61 \pm 7.3 \% ; p=0.6172$ ) (Figure 3.6 A right) indicates the compensatory nature of this HT where blood flow is sustained.

NGS of RNA isolated from Sham and TAC animals one week after surgery followed by differential gene expression analysis identified 217 differentially expressed genes, 144 being upregulated and 73 being downregulated (log2FC $\geq 1$ or $\leq-1$, Padj $\leq 0.05$ ) (Figure 3.6 B). GO terms associated with differentially expressed genes include 'regulation of cartilage development', 'collagen fibril organization', 'cardiac muscle hypertrophy' and 'striated muscle adaptation', the latter two terms associated with hypertrophic growth (Figure 3.6 C) (Nomura et al. 2018). 
A

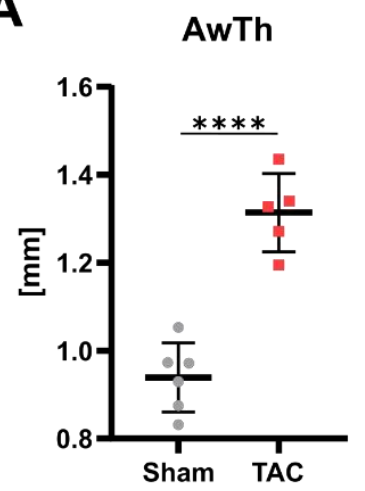

EF

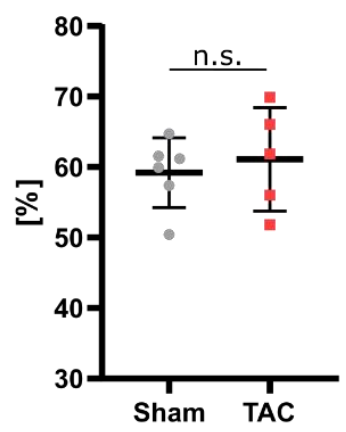

B

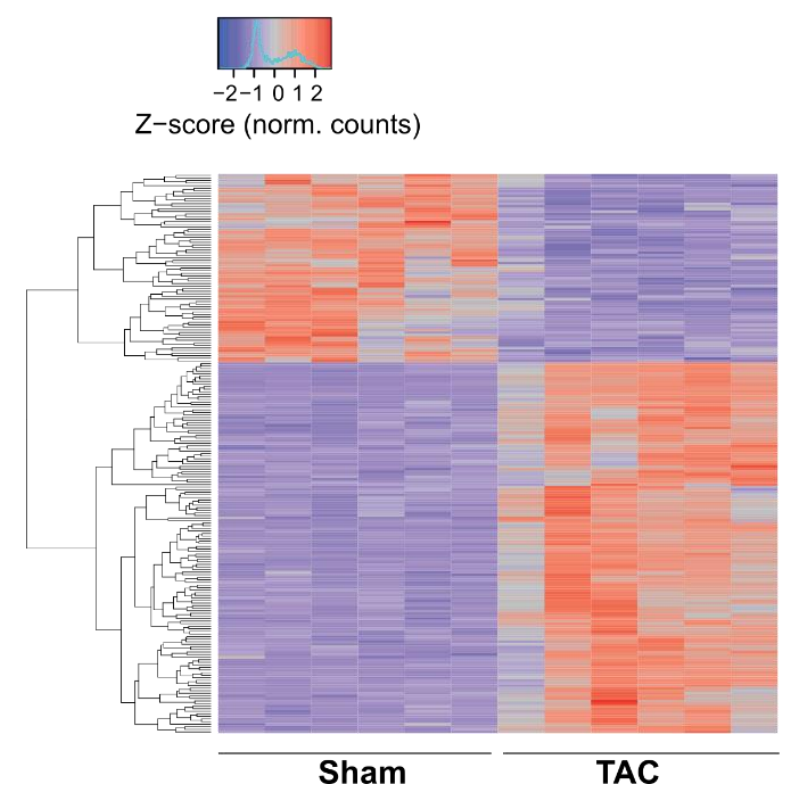

C

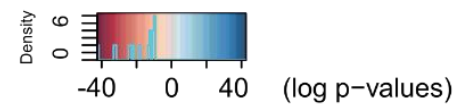

wound healing

regulation of cartilage development

collagen fibril organization

cardiac muscle hypertrophy

negative regulation of plasminogen activation

striated muscle adaptation

response to amino acid

podosome assembly

hyaluronan metabolic process

endodermal cell differentiation

collagen-activated tyrosine kinase receptor signaling pathway

extracellular fibril organization

homotypic cell-cell adhesion

Figure 3.6 Cardiac hypertrophy is observed one week after TAC with a change in gene expression

(A) Echocardiographic analysis of mouse hearts one week after TAC surgery shows an increase of the anterior wall thickness (AwTh) by $0.37 \mathrm{~mm}(p=0.0001)$ with a sustained ejection fraction (EF) (Sham: $59 \pm 5.0 \%$, TAC: $61 \% \pm 7.3 ; p=0.6172$ ), typical for compensated hypertrophy. An unpaired t-test was performed, and the values are represented with the error bar indicating the standard deviation (SD). Each dot represents one animal, with a total $n=6$ for Sham and $n=5$ for TAC. (B) Differential gene expression of RNA-Seq data (log2FC $\geq 1$ or $\leq-1$, Padj $\leq 0.05$ ) from hypertrophic mouse heart tissue revealed 144 upregulated, and 73 downregulated genes. (C) The heatmap represents the most significant GO categories found for the 217 differentially expressed genes, most of which belong to typical hypertrophic response pathways. 


\subsubsection{Persistent TAC-induced pressure overload progresses to heart failure}

Persistent stress by the PO should lead to HF progression in TAC challenged mice over time characterized by an increased wall thickness but reduced EF. When analyzing a time-point 8weeks post-TAC, a decompensation of the heart occurs as seen from the echocardiographic analysis (Figure 3.7 A). The LV is still hypertrophic as demonstrated by increased AwTh (1.351 $\pm 0.1 \mathrm{~mm}$ in TAC as compared to Sham with $0.983 \pm 0.07 \mathrm{~mm} ; p=0.0001$ ), while the EF is $22 \%(p=0.0002)$ reduced in TAC animals compared to Sham animals (corresponding to the difference of $13 \%$ from Sham: $59 \pm 4.7 \%$ to TAC: $46 \pm 5.4 \%$ ).

Gene expression analysis shows that 174 genes were differentially expressed, with 91 upregulated and 83 downregulated genes $(\log 2 \mathrm{FC} \geq 1 ; p \leq 0.05)$ (Figure $3.7 \mathrm{~B})$. Among the top GO terms associated with the differentially regulated genes are 'positive regulation of cardiac muscle contraction' and 'positive regulation of heart contraction', indicating that cardiac function is affected. Other associated terms include 'collagen fibril organization', 'cell-cell junction organization', and 'actin filament bundle assembly', all terms which may be assigned to cardiac plasticity. 
A

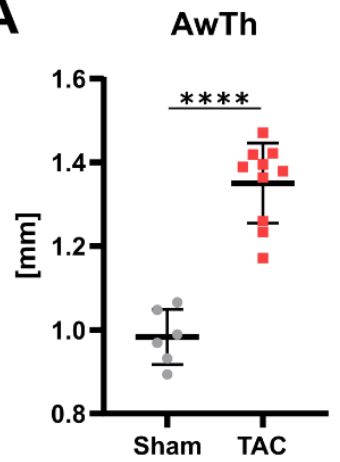

EF

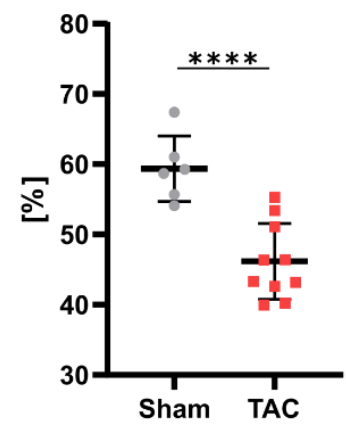

B

C

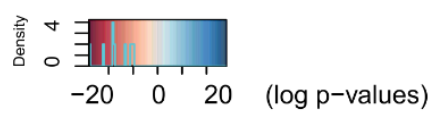

Z-score (norm.counts)

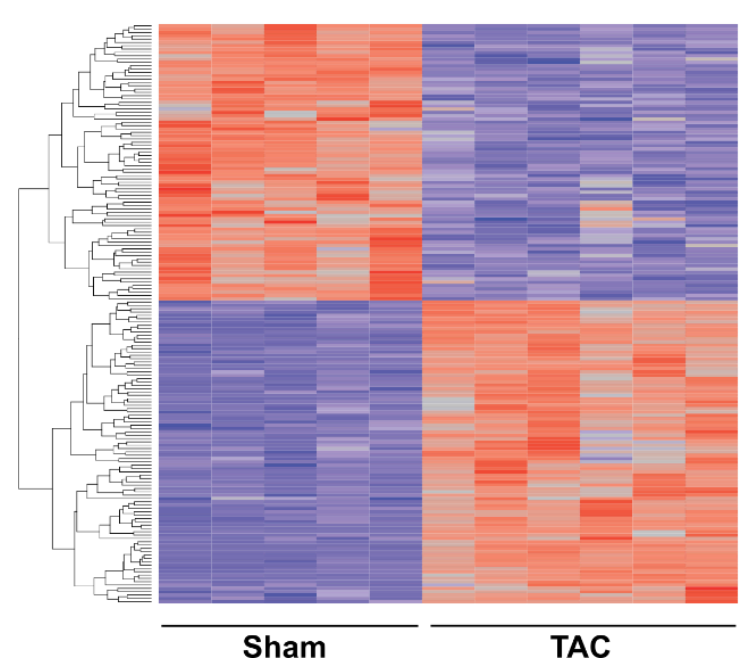

skeletal muscle adaptation negative regulation of plasminogen activation positive regulation of cardiac muscle contraction positive regulation of heart contraction negative regulation of smooth muscle cell proliferation response to oxygen levels collagen fibril organization cell-cell junction organization actin filament bundle assembly

Figure 3.7 Mice develop heart failure with gene expression changes 8-weeks post-TAC

(A) Echocardiographic analysis of mice eight weeks after TAC surgery shows a sustained increased anterior wall thickness (AwTh) by $0.37 \mathrm{~mm}(p=0.0001)$ with a decreased ejection fraction (EF) (Sham: $59 \pm 4.7 \%$, TAC: $46 \pm 5.4 \% ; p=0.0002$ ), both typical attributes of heart failure. Each dot represents one animal with $n=6$ for Sham, $n=10$ for TAC, and error bars indicate SD. (B) Differential gene expression of RNA-Seq data $(\log 2 F C \geq 1$ or $\leq-1$, Padj $\leq 0.05)$ from mouse failing heart tissue revealed 91 upregulated genes, and 83 downregulated genes. (C) The heatmap represents the most frequent GO categories found for differentially expressed genes, which demonstrate that metabolic and cardiac function pathways are affected. 
A comparison of the differentially deregulated genes identified in mouse cardiac HT and HF reveals, that 71 genes are commonly deregulated in both states of the disease (Figure 3.8 left). Gene enrichment analysis (Figure 3.8 right) demonstrates that these commonly deregulated genes participate in GO terms of important cardiac stress-response pathways, e.g. 'muscle hypertrophy in response to stress' is the top identified pathway. Furthermore, GO-identified pathways include 'positive regulation of heart contraction', 'collagen fibril organization', and 'cardiac muscle tissue morphogenesis', all of which represent pathways of cardiac plasticity regulation.

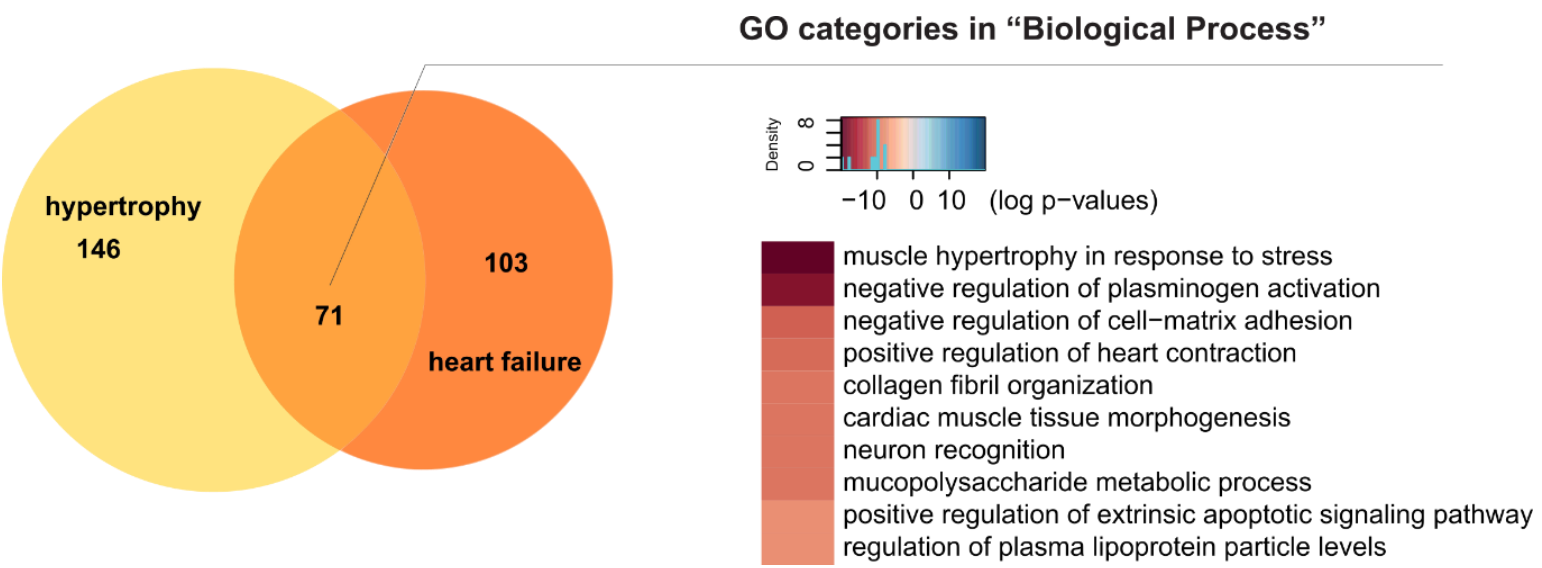

Figure 3.8 Select genes are continuously deregulated during the progression from hypertrophy to heart failure and are associated with pathways of cardiac plasticity The Venn diagram shows genes differentially expressed in hypertrophy and heart failure, with an overlap of 71 genes deregulated in both states. GO term analysis of these 71 genes reveals, that they are cardio-specific and mainly linked to structural and cardiac plasticity pathways. 


\subsubsection{Human end-stage heart failure}

In order to investigate the transcriptome, as well as epitranscriptome in failing human hearts, RNA isolated from human end-stage heart failure (HF) tissue was analyzed and compared to RNA isolated from non-failing (NF), healthy heart tissue via MeRIP followed by NGS. It was not possible to analyze the compensated hypertrophic state in humans for this project as, even though tissue biopsies from hypertrophic hearts do exist, the amount of tissue needed for subsequent MeRIP is neither sufficient nor feasible to attain. However, sufficient tissue from end-stage HF patients was available due to ex-plantation of the heart in the late stage of the disease or tissue becoming available after HF-related deaths.

Investigation of transcriptomic changes in human end-stage HF shows that 228 genes are differentially deregulated, comprising of 73 upregulated genes and 155 downregulated genes (Figure $3.9 \mathrm{~A}$ ).

Genes which are deregulated play roles in cardiac plasticity, as indicated by the assigned GO terms (Figure 3.9 B). Here, pathways including 'extracellular matrix organization', 'regulation of cell-substrate adhesion', and 'actomyosin structure organization' are among the top enriched terms. Similar pathways have been reported in recent RNA-seq studies (Sweet et al. 2018).

A

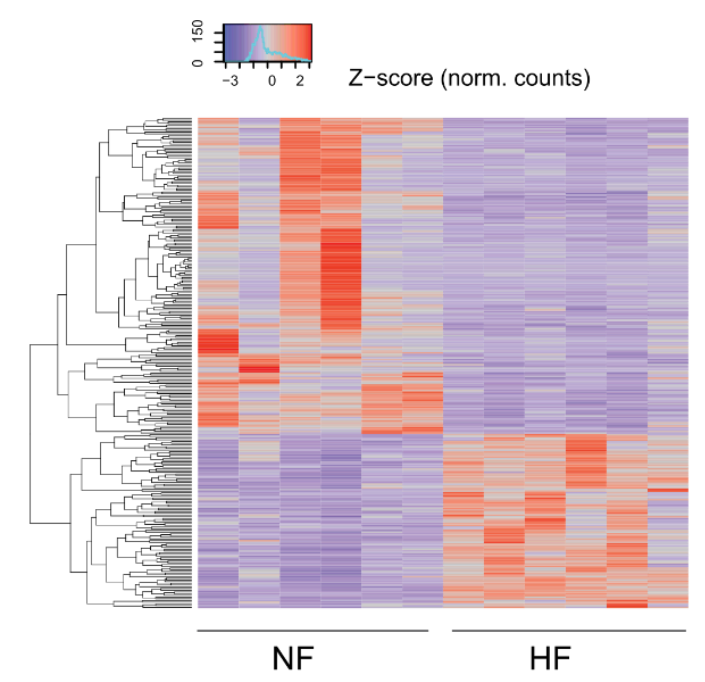

B

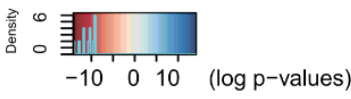

regulation of endothelial cell apoptotic process negative regulation of hemostasis extracellular matrix organization leukocyte migration response to hydrogen peroxide positive regulation of cell-substrate adhesion response to ketone actomyosin structure organization amino acid transport regulation of smooth muscle cell proliferation response to tumor necrosis factor

Figure 3.9 Gene expression changes in human end-stage heart failure

(A) 228 genes were differentially expressed in human end stage heart failure (HF) with 73 genes upregulated and 155 downregulated genes. (B) The heatmap represents the frequency of GO terms assigned to those deregulated genes. Pathways related to cardiac plasticity were among the top GO terms. 


\subsubsection{Methylation changes in the progression to heart failure}

After the analysis of expression changes in response to cardiac stress on the transcriptomic level, the effects of cardiac stress on the epitranscriptomic level were investigated by analyzing m6A RNA methylation changes. This was done by MeRIP followed by NGS (MeRIP-seq). By comparing immunoprecipitated (Iped) samples to the total RNA (Input) used in transcriptome analysis, the methylation of transcripts could be detected. By further comparing the methylation levels in TAC or end-stage HF samples to Sham or NF human heart samples, methylation changes could be calculated. Transcripts with more methylation peaks in TAC or HF tissue compared to Sham or NF heart tissue are called hypermethylated, those with fewer peaks hypomethylated.

\subsubsection{Methylation changes in mouse hypertrophy}

To assess epitranscriptomic changes in the compensated $\mathrm{HT}$, the time point of one week postTAC was investigated (Figure 3.10). When applying the same cut-offs for deregulation to differentially methylated transcripts as well as differentially expressed transcripts $(\log 2 \mathrm{FC} \geq 1$ or $\leq-1$, Padj $\leq 0.05$ ) it shows that many more transcripts were altered on the methylation level (1638 transcripts) than on the expression level (217 transcripts) (Figure 3.10 A left panel). The methylation changes of transcripts mainly occurred in the CDS and 3' UTR, where most hypoas well as hypermethylated m6A peaks were detected (Figure 3.10 A right panel).

A comparison between differentially methylated and differentially expressed genes revealed an overlap of 78 transcripts which were present in both states (Figure 3.10 B), indicating a possible effect of methylation on the expression level for these genes. Further characterization by $\mathrm{GO}$ term analysis indicates, that they mainly participate in structural pathways involved in remodeling processes, e.g. 'extracellular fibril organization', 'collagen fibril organization', and 'atrioventricular valve morphogenesis' and cell differentiation pathways. 'Collagen activated tyrosine kinase receptor signaling pathway' is the only signal transduction GO term detected, which may relate to the other collagen reorganization pathways detected for these transcripts. 


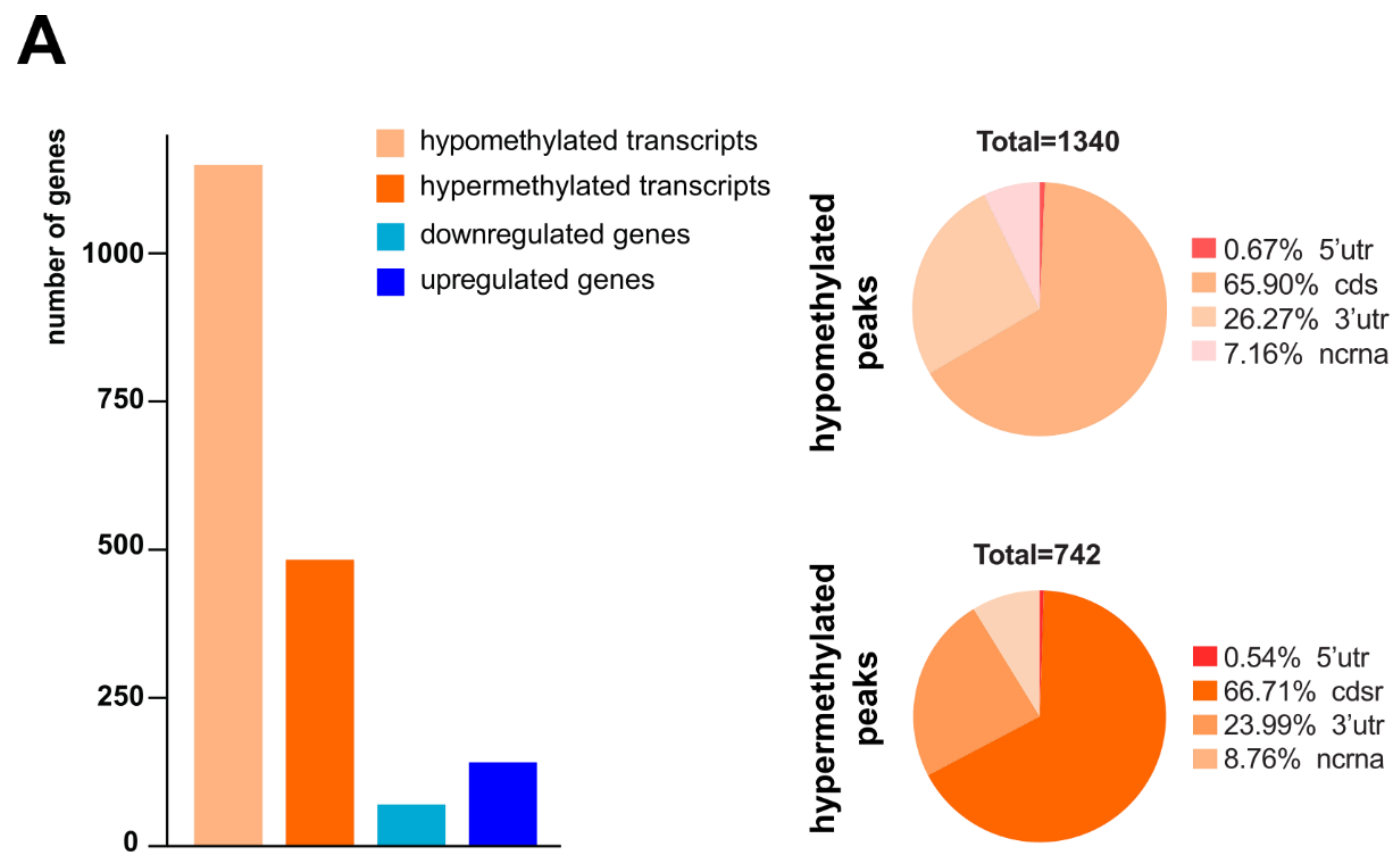

B

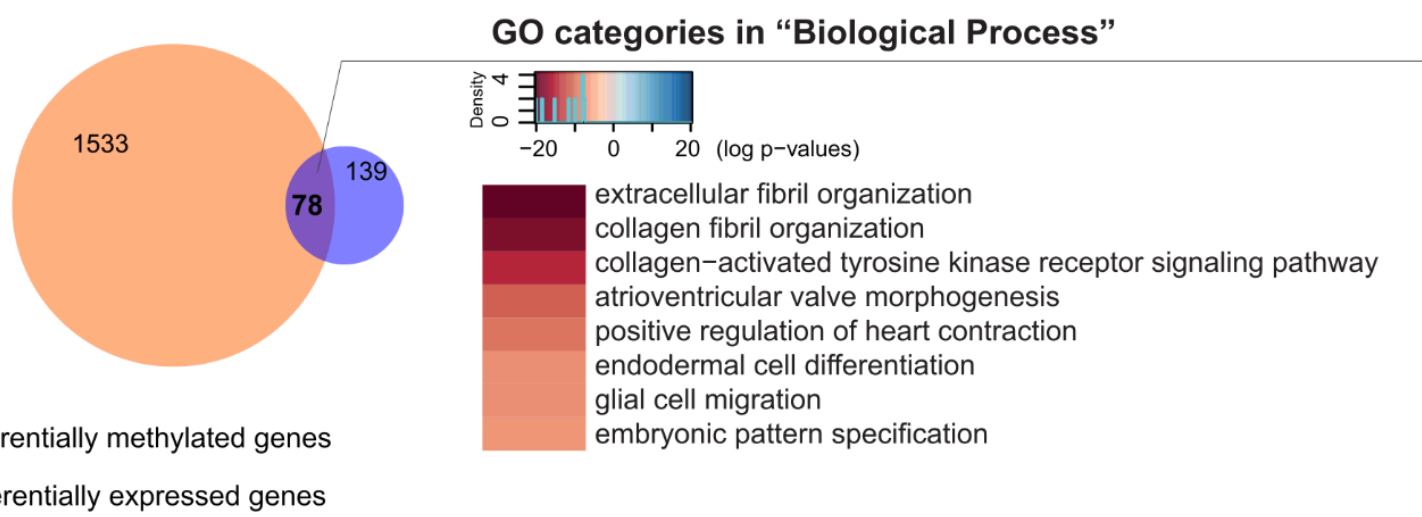

Figure 3.10 m6A methylation changes outnumber expression changes in mouse hypertrophy (A) Left panel: The bar chart shows the total number of genes with altered methylation or expression levels. Right side: The pie charts represent detected m6A peaks from which methylation deregulated transcripts were determined. They demonstrate at which sites transcripts were differentially hypo(upper panel) or hyper-methylated (lower panel), indicating that methylation was mainly changed in the 3' UTR and the CDS upon hypertrophy. (B) The Venn diagram shows which genes were differentially methylated or differentially expressed (log2FC $\geq 1$ or $\leq-1$, Padj $\leq 0.05$ ), with an overlap of 78 genes being differentially methylated and expressed at the same time. The right panel shows the GO term analysis of these 78 genes, consisting of pathways which are mostly of a structural nature, indicating a connection to remodeling processes.

Having analyzed GO terms that differentially expressed transcripts (Figure 3.6 C) code for, as well as GO terms that transcripts being differentially expressed and differentially methylated at the same time in HT are associated with (Figure 3.10 B), it was then of interest to analyze the pathways that transcripts changed only at the methylation level code for (Figure 3.11). Interestingly, mainly GO terms of metabolic, catabolic and especially signal transduction 
pathways were found for both, hypo- (Figure 3.11 A) as well as hypermethylated transcripts (Figure 3.11 B).

A

\section{hypomethylated transcripts}

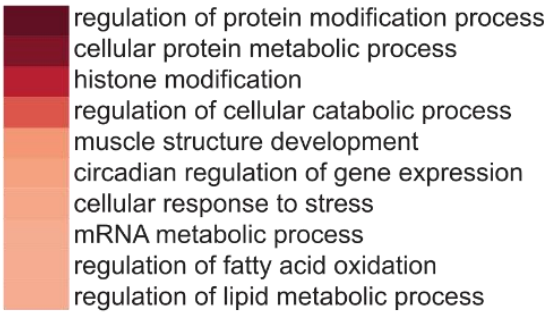

B

\section{hypermethylated transcripts}

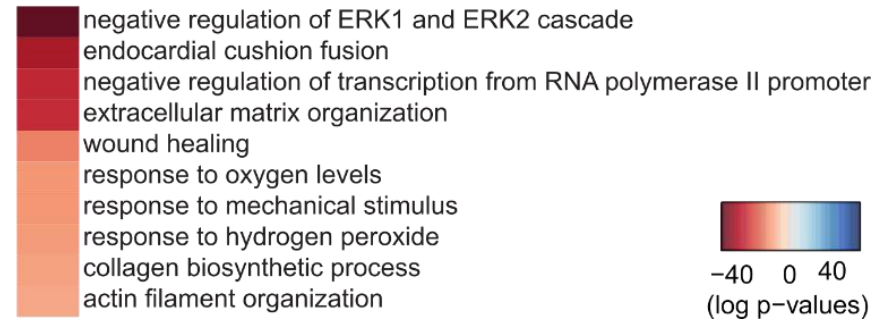

Figure 3.11 Differentially methylated transcripts in hypertrophy are associated with catabolic and metabolic processes as well as signal transduction pathways

GO term analysis was performed on differentially methylated transcripts which have unchanged expression levels in mouse cardiac hypertrophic tissue. The heatmaps show GO pathways according to their occurrence in (A) hypomethylated and (B) hypermethylated transcripts. 


\subsubsection{Methylation changes in mouse heart failure}

RNA methylation analysis of mouse hearts 8-weeks post-TAC in the HF state demonstrates notable changes of m6A levels in failing tissue compared to healthy tissue. As observed in HT tissue from mice (Figure 3.10), methylation changes outnumber expression changes with 1215 transcripts being differentially methylated and only 174 being differentially expressed (Figure 3.12 A left panel). The methylation changes of transcripts mainly occurred in the 3' UTR, where most hypo- as well as hypermethylated m6A peaks were detected (Figure 3.12 A right panel).

A total of 47 genes are differentially expressed and differentially methylated at the same time (Figure 3.12 B). GO term analysis of these transcripts (Figure 3.12 B) shows that they are associated with GO terms related to cardiac plasticity changes, including 'regulation of smooth muscle cell migration', 'regulation of heart contraction', 'cell adhesion mediated by integrin', and 'cardiac muscle tissue growth', as well as collagen processes. The 'regulation of cellular response to transforming growth factor $ß$ stimulus' pathway, although being a signaling related pathway, may be directly linked to growth changes of the heart, therefore pointing to cardiac plasticity effects also found in genes only altered at the expression level (Figure 3.7 B). 
A
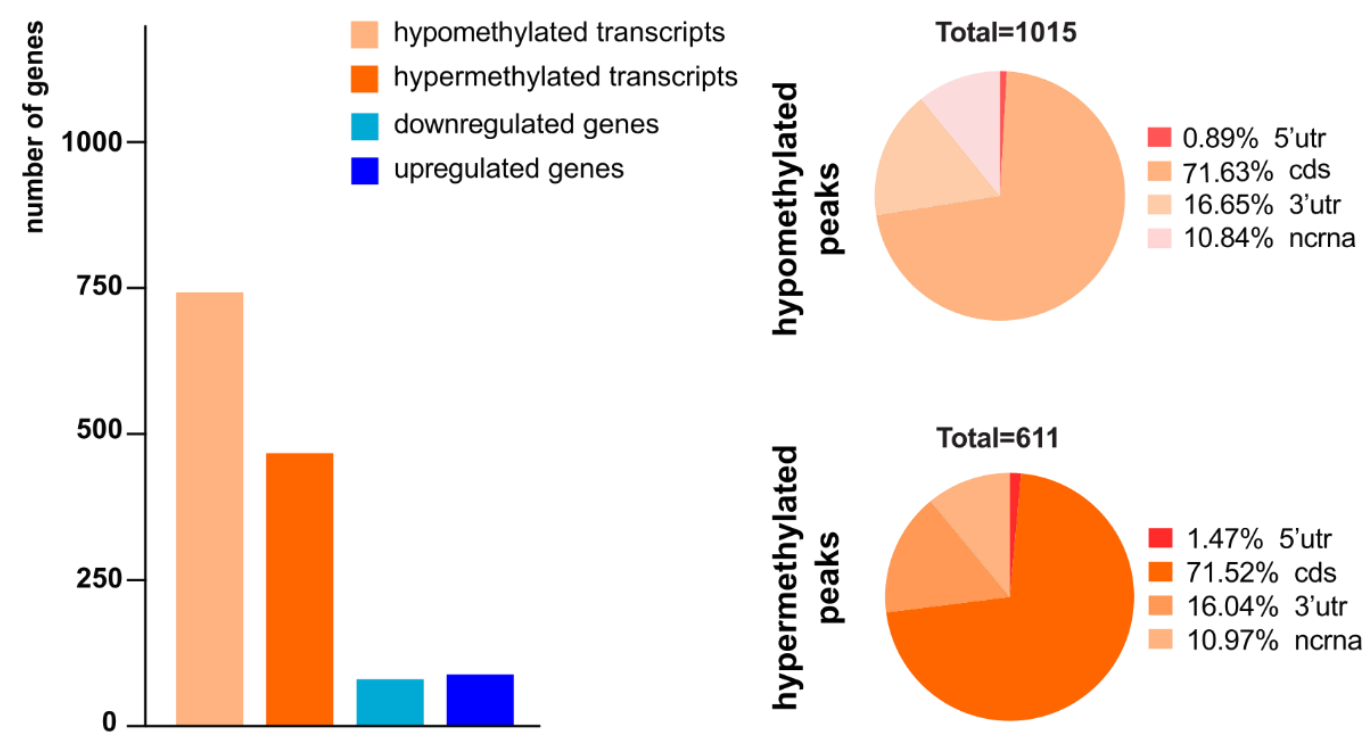

B

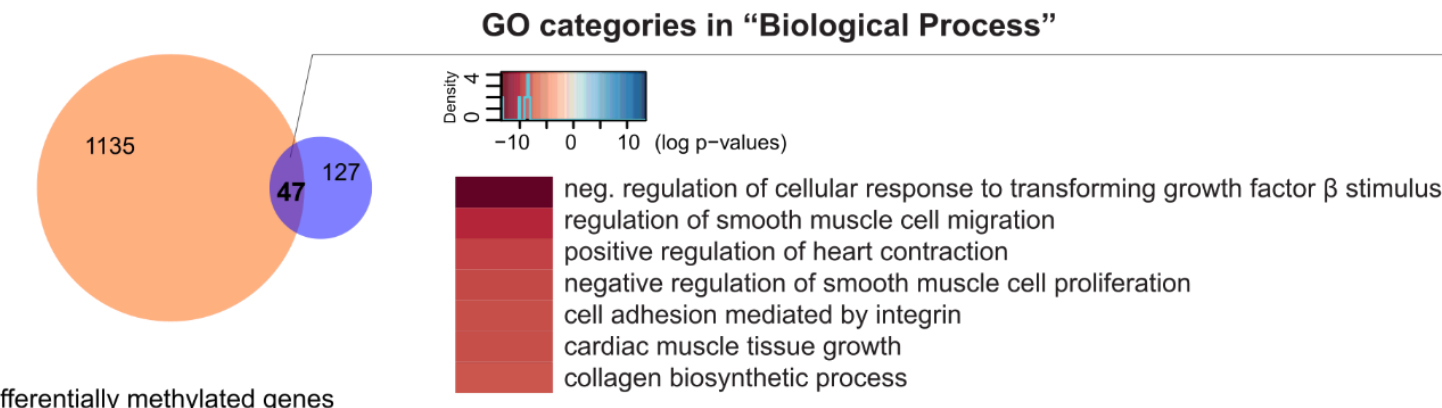

differentially methylated genes

differentially expressed genes

Figure $3.12 \mathrm{~m} 6 \mathrm{~A}$ methylation changes outnumber expression changes in mouse heart failure (A) Left panel: The bar chart shows the total number of genes with altered methylation or expression levels in mouse heart failure. Right side: The pie charts represent detected m6A peaks from which methylation deregulated transcripts were determined. They demonstrate at which sites transcripts were differentially hypo- (upper panel) or hyper-methylated (lower panel), indicating that methylation was mainly changed in the 3' UTR upon HF. (B) The Venn diagram shows differential methylation or expression of genes, with an overlap of 78 genes being both differentially methylated and expressed at the same time. The right panel shows the GO term analysis of these 78 genes; identified pathways are mostly of structural nature, suggesting a role of these genes in remodeling processes.

GO term analysis of genes which are exclusively altered at the methylation level (Figure 3.13) reveals that hypomethylated transcripts (Figure 3.13 A) are found in GO terms of metabolic and catabolic processes, including 'protein catabolic processes', 'phosphate-containing compound metabolic processes', and 'regulation of cellular metabolic processes', a comparable GO term readout as observed in the hypertrophic state (Figure 3.11 A). GO terms associated with hypermethylated transcripts (Figure $3.13 \mathrm{~B}$ ) show a relation to catabolic and metabolic processes (i.e. 'collagen biosynthetic process' or cellular protein metabolic processes'), as well 
as signal transduction pathways including 'negative regulation of signal transduction', and 'Rho protein signal transduction'. In addition, GO terms associated with cardiac plasticity are also detected, specifically the different developmental and organization pathways.

A

\section{hypomethylated transcripts}

oxidation-reduction process

mitochondrial transport

protein catabolic process

coenzyme biosynthetic process

phosphate-containing compound metabolic process

cellular biosynthetic process

positive regulation of cellular metabolic process

heart development
B hypermethylated transcripts

cardiac muscle tissue development
muscle tissue development
endocardial cushion fusion $\quad \begin{array}{ll}\text { extracellular matrix organization } & -20 \\ \text { negative regulation of signal transduction } \\ \text { Rho protein signal transduction } \\ \text { connective tissue development } \\ \text { blood vessel morphogenesis } \\ \text { negative regulation of cellular protein metabolic proces: } \\ \text { collagen biosynthetic process }\end{array}$

Figure 3.13 Differentially methylated transcripts in heart failure are associated with catabolic and metabolic processes as well as signal transduction pathways

GO term analysis was performed for differentially methylated transcripts with unaltered expression level in mouse heart failure. The heatmaps show GO pathways according to their occurrence in (A) hypomethylated and (B) hypermethylated transcripts. 


\subsubsection{Methylation changes in human end-stage heart failure}

Having investigated the epitranscriptomic changes in mouse HT and HF, RNA methylation changes were analyzed in human HF tissue as well. As described before, ex-plantation of hearts and hence tissue availability only occurs with end-stage HF patients and not patients with hypertrophic hearts. Therefore, analysis of cardiac HT in human tissue was not feasible. However, the investigation of human end-stage HF samples (Figure 3.14) demonstrates some intriguingly findings. Similar as seen with mouse HF tissue (Figure 3.12), methylation changes occurring in the diseased state of humans outnumber expression changes by far. 1246 transcripts were altered at the methylation level and 228 were differentially expressed (Figure 3.14 A left panel) in HF tissue compared to healthy NF tissue. These methylation changes occur mainly in the CDS and 3' UTR as indicated by detected hypo- and hypermethylated m6A peaks making up for the methylated transcripts (Figure 3.14 A right panel).

Only 30 transcripts which showed changes in both methylation and expression level were detected (Figure 3.14 B), and were associated with GO terms including 'positive regulation of tumor necrosis factor production', and 'muscle hypertrophy in response to stress'. Similar observations were made for mouse HF tissue, where transcripts being deregulated at the expression and methylation level at the same time were also associated with GO terms of hypertrophic growth (Figure 3.12 B). 


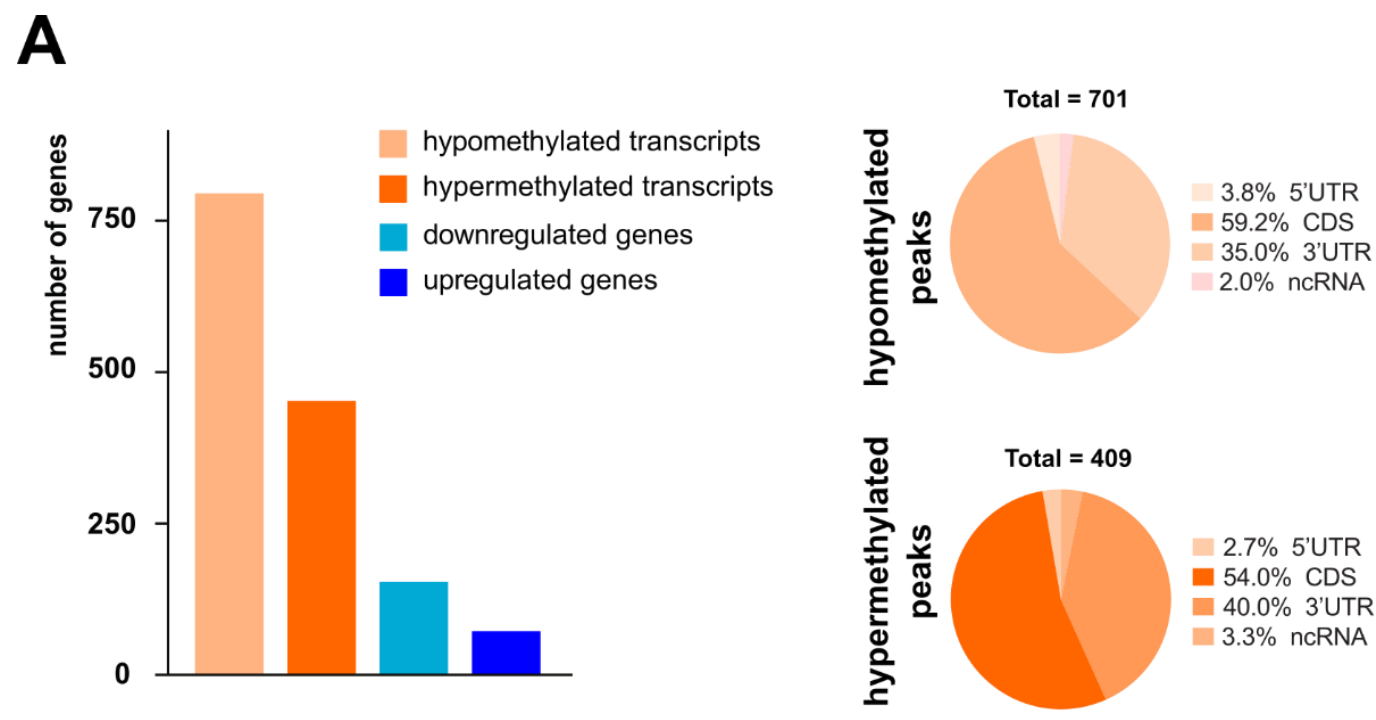

B

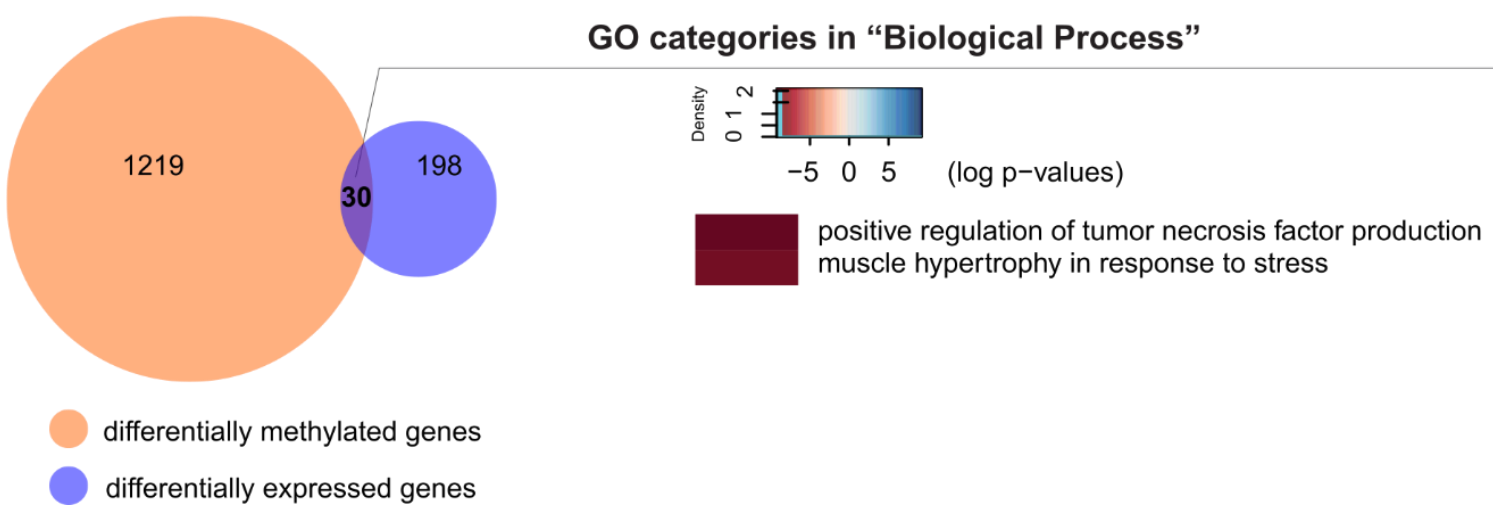

Figure 3.14 Methylation changes outnumber expression changes upon heart failure in human tissue

(A) The bar chart shows the total number of genes with altered methylation or expression levels (left panel). The pie charts demonstrate to what extent transcripts were differentially hypo- or hypermethylated at different sites along the transcript and show that methylation was mainly changed in the CDS and the 3' UTR of transcripts isolated from human HF tissue. (B) The Venn diagram shows differentially methylated and expressed transcripts with an overlap of 30 genes. The right panel shows the GO term analysis of these 30 genes; most identified GO pathways are related to hypertrophy and remodeling processes.

A more detailed investigation of transcripts only differentially methylated with unaltered expression level by GO term analysis (Figure 3.15) indicates that hypomethylated transcripts participate in signal transduction pathways such as 'negative regulation of signal transduction', and 'adrenergic receptor signaling pathway involved in heart process' (Figure 3.15 A), and hypermethylated transcripts are associated with terms including 'response to growth factor' and 'response to muscle stretch' (Figure 3.15 B). Furthermore, GO terms of catabolic and metabolic processes show up in both hypo- and hypermethylation datasets, including 'regulation of proteolysis' and 'regulation of protein catabolic process' identified in the hypomethylation dataset and 'glycoprotein metabolic process' identified in the 
hypermethylation dataset. An overrepresentation of genes coding for signal transduction processes was also observed in differentially methylated genes in the HF tissue of mice (Figure 3.13).

A

\section{hypomethylated transcripts}

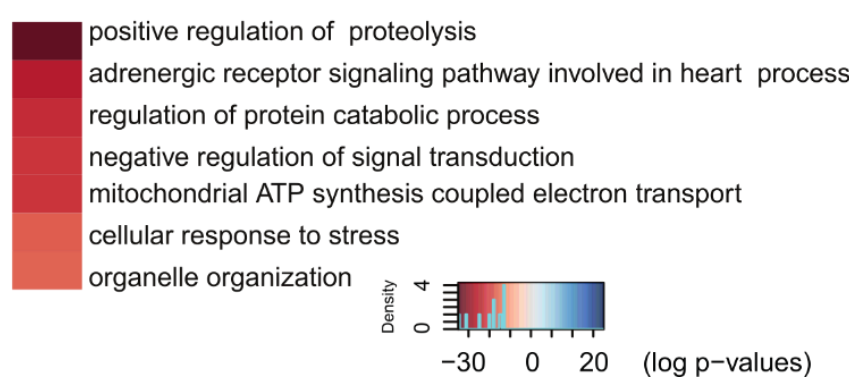

B

\section{hypermethylated transcripts}
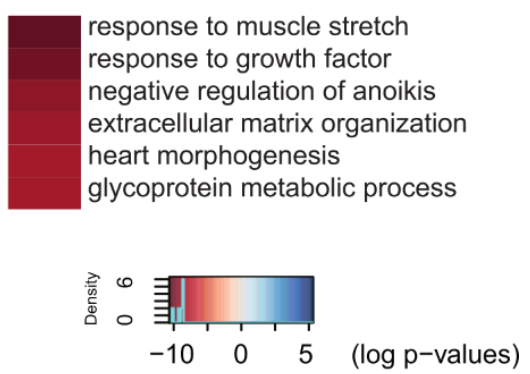

Figure 3.15 Differentially methylated transcripts in human heart failure are implicated in signal transduction pathways

GO term analysis was performed for differentially methylated transcripts with unaltered expression levels in human end-stage heart failure. The heatmaps show GO pathways according to their occurrence in (A) hypomethylated and (B) hypermethylated transcripts. 


\subsubsection{Comparison of the mouse and human heart failure epitranscriptome}

To further compare m6A methylation changes in heart failure between human and mouse tissue (Figure 3.16), 1604 differentially methylated transcripts from 8-weeks post-TAC heart failure mouse tissue were compared to 1392 identified differentially methylated transcripts from human end-stage heart failure tissue and 403 transcripts identified in both datasets. GO terms assigned to these commonly methylated transcripts (Figure 3.16) are associated with cardiac specificity, as demonstrated by terms like 'contractile actin filament bundle assembly', 'cardiac vascular smooth muscle cell differentiation', and 'regulation of cardiac muscle contraction by release of sequestered Ca2+ ion'. The similarity of the associated GO terms and overlapping transcripts may indicate that the m6A RNA methylation process in HF is comparable between mouse and human.

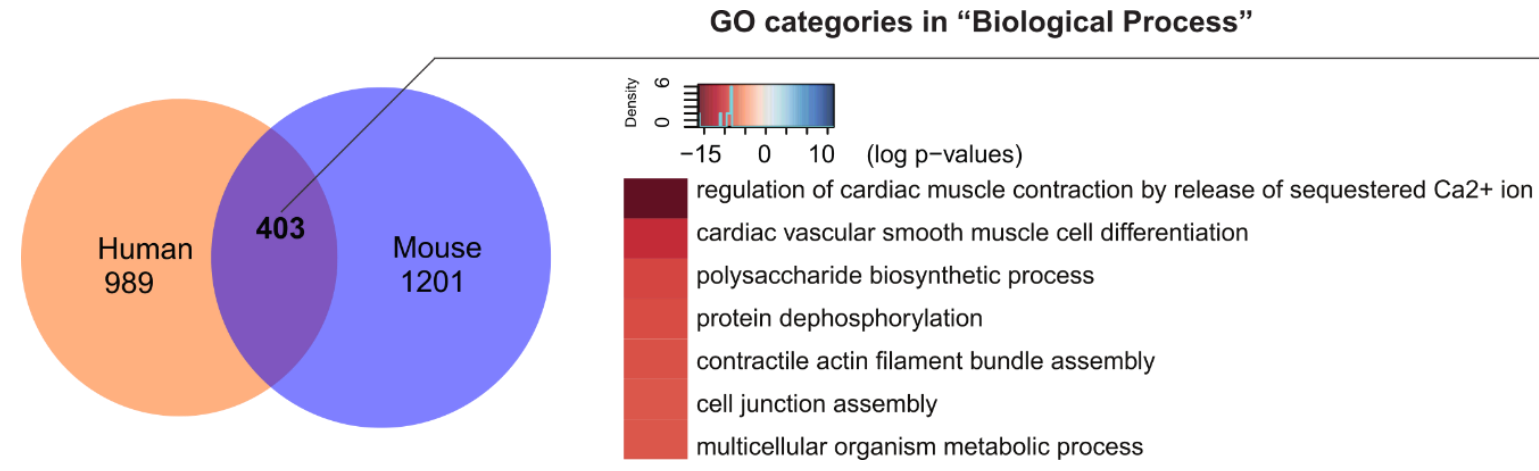

Figure 3.16 Many differentially methylated transcripts in heart failure tissue of mouse and human are of cardiac specificity

All differentially methylated transcripts found in TAC induced mouse heart failure and human endstage heart failure were compared, and the Venn diagram demonstrates an overlap of 403 transcripts found in both tissues (representation factor 7.8, $p<3.851 \mathrm{e}-251$ ). GO term analysis was performed on the commonly differentially methylated transcripts, and the pathways are shown as a heatmap according to their occurrence. Identified GO terms are cardio-specific, indicating the presence of $\mathrm{m} 6 \mathrm{~A}$ methylation in heart failure response in both mouse and humans.

In healthy heart tissue, the fraction of transcripts carrying methylation marks were associated with GO terms of energy conservation mechanisms such as mitochondrial function and catabolic processes when methylated in the 5' UTR, or signal transduction mechanisms when methylated in the 3' UTR (Figure 3.4 A, C). Transcripts with m6A methylation in the 3' UTR also code for proteins found in GO terms of developmental and tissue differentiation mechanisms.

In response to cell stress, in this study TAC surgery or end-stage HF, genes that were changed at the expression level were found associated with GO terms of cardiac structure and cardiac plasticity pathways, indicating that they may be important in cardiac remodeling (Figure $3.6 \mathrm{C}$, Figure 3.7 C, Figure 3.9 B). 
Transcripts altered at both the methylation as well as the expression level in response to stress were associated with hypertrophic changes and cellular growth (Figure 3.10 B, Figure 3.12 B, Figure 3.14 B)., similar to genes only differentially expressed. This may support the assumption asserted in previous studies that methylation affects expression directly by regulating mRNA stability and decay (Wang et al. 2014; Ke et al. 2017). In my study, especially genes associated with GO terms of response to cell stress are affected by methylation and expression.

However, a majority of transcripts were exclusively changed at the methylation level in $\mathrm{HT}$ and HF tissue compared to healthy tissue. These transcripts were mainly associated with pathways of signal transduction, response to remodeling and modified cell stress, and metabolic and catabolic processes, demonstrating that cellular processes affected by m6A RNA methylation may be substantially different from those influenced by RNA expression changes. 


\subsubsection{A potential effect of methylation on translation}

3.1.4.1 Methylation-mediated expression level changes may not be the main mechanism of translation control

RNA methylation is associated with altered mRNA decay rates and stability (Wang et al. 2014; Shi $\mathrm{H}$ et al. 2017), but in response to cardiac stress, a vast majority of transcripts were only affected at the methylation level while the expression level was unchanged. Therefore, altering mRNA stability is likely not the main mechanism by which m6A methylation affects mRNA expression and translation during cardiac $\mathrm{HT}$ and the progression to HF.

To see if RNA methylation potentially affects mRNA stability and hence the transcript level in healthy cardiac tissues, a correlation analysis of the methylation and expression level was performed considering those transcripts that were differentially methylated upon HF. Neither in mouse (Figure 3.17 A), nor in human heart tissue (Figure 3.17 B), the expression level of transcripts correlated with their respective methylation level in healthy heart tissue.

A

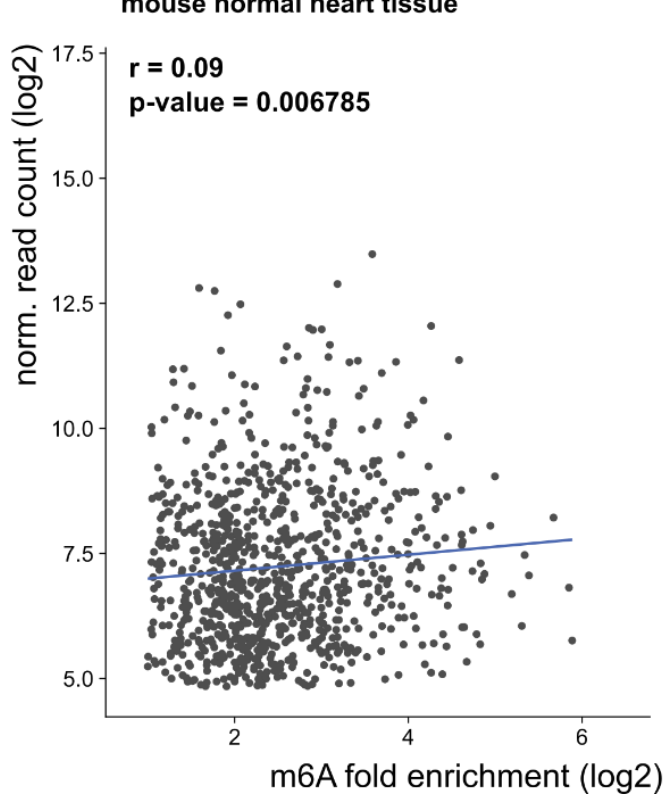

B

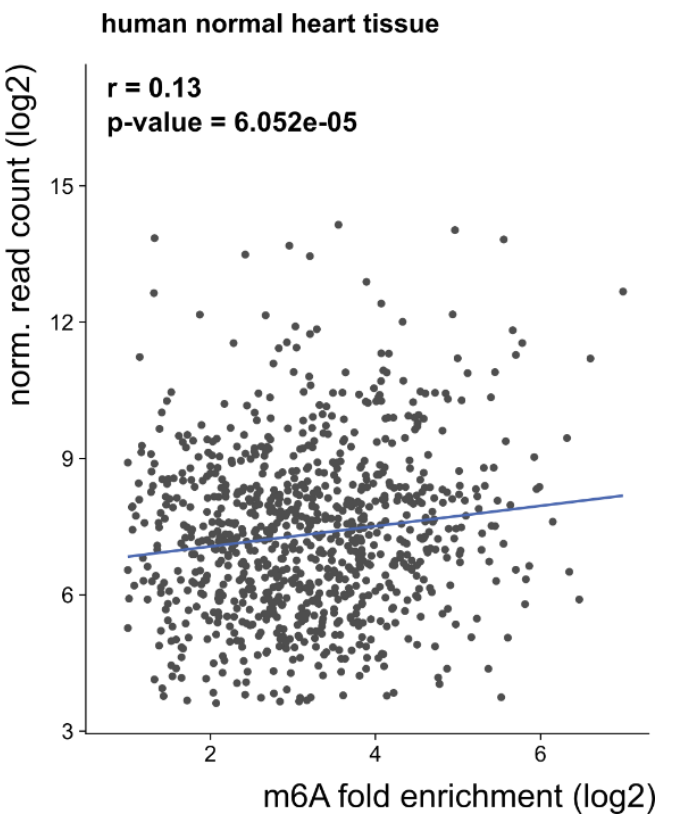

Figure 3.17 Transcripts changed at the methylation level are most likely different from transcripts changed at the expression level

Correlation analysis between the m6A methylation level and the respective expression level of transcripts reveals no significant correlation in healthy mouse or human heart tissue. Only those transcripts found differentially methylated in HF tissue were analyzed to see if a potential effect of m6A methylation on mRNA stability and hence transcript level exists for these transcripts in healthy heart tissue. 
While RNA methylation has been associated with altered mRNA stability and decay (Wang et al. 2014; Shi H et al. 2017), this mechanism of transcriptional and translational control does not appear to be valid in cardiac tissue and in HT or HF. This suggests that m6A performs other functions in these tissues.

\subsubsection{The effect of RNA methylation on translation by polysomal analysis}

Previous studies have suggested that m6A RNA methylation may affect translation by altering ribosomal occupancy (Bodi et al. 2015) or translation initiation (Meyer et al. 2015; Li A et al. 2017). To asses if the many methylation changes observed in this study may affect translation, polysomal profiling was applied (Chassé et al. 2017). A group or cluster of ribosomes, all bound to the same mRNA, is called polysome. Generally, the ribosome density at a certain mRNA is proportionally correlated with the translation rate of this mRNA. The more ribosomes are attached, the higher the rate of translation. Therefore, transcripts found in polysomal fractions are highly translated. The analysis of ribosome-dense (polysomal) mRNAs that are differentially methylated but unchanged at transcript level in heart failure allows to draw conclusions on the impact of m6A RNA methylation on translation.

Polysome profiling (Chassé et al. 2017) was performed with mouse HF tissue (8-weeks postTAC) to identify transcripts which are actively translated and further compared to the methylation data collected for similar tissues. To better elucidate the potential effect of methylation on translation, only transcripts were considered which were initially unchanged at their expression level (differentially methylated and not differentially expressed). This excludes transcripts up- and downregulated at the expression level which would otherwise be found in polysomes based on their abundance.

225 transcripts were identified which were significantly enriched or depleted in the polysomal fractions (cut-off: foldchange $\geq 2$ for enriched, foldchange $\leq 0.5$ for depleted, Padj $\leq 0.05$ ) (Figure 3.18)

Intriguingly, a GO term analysis of the detected transcripts (Figure 3.18) reveals, that mRNAs depleted from the polysomal fraction, being therefore translated to a lesser extent, are found in GO terms of metabolic and catabolic processes, including 'fructose metabolic process', 'fatty acid catabolic process', and biosynthetic processes. Similar terms were also identified in hypomethylated transcripts (Figure 3.13 A).

Furthermore, mRNAs enriched in polysomes, therefore being translated to a higher extent, are associated with GO terms of development pathways (e.g. 'sclerotome development') and proliferative pathways, such as 'regulation of actin filament bundle assembly', and 'stem cell division', as well as signal transduction pathways including 'regulation of calcium-mediated 
signaling' or apoptotic signaling pathways. Intriguingly, hypermethylated transcripts were found to have similar GO term associations (Figure 3.13 B). Also, enriched mRNAs are coding for proteins involved in pathways of smooth muscle cell regulation ('regulation of smooth muscle cell migration'), GO terms similarly identified for commonly differentially methylated transcripts from mouse and human (Figure 3.16).

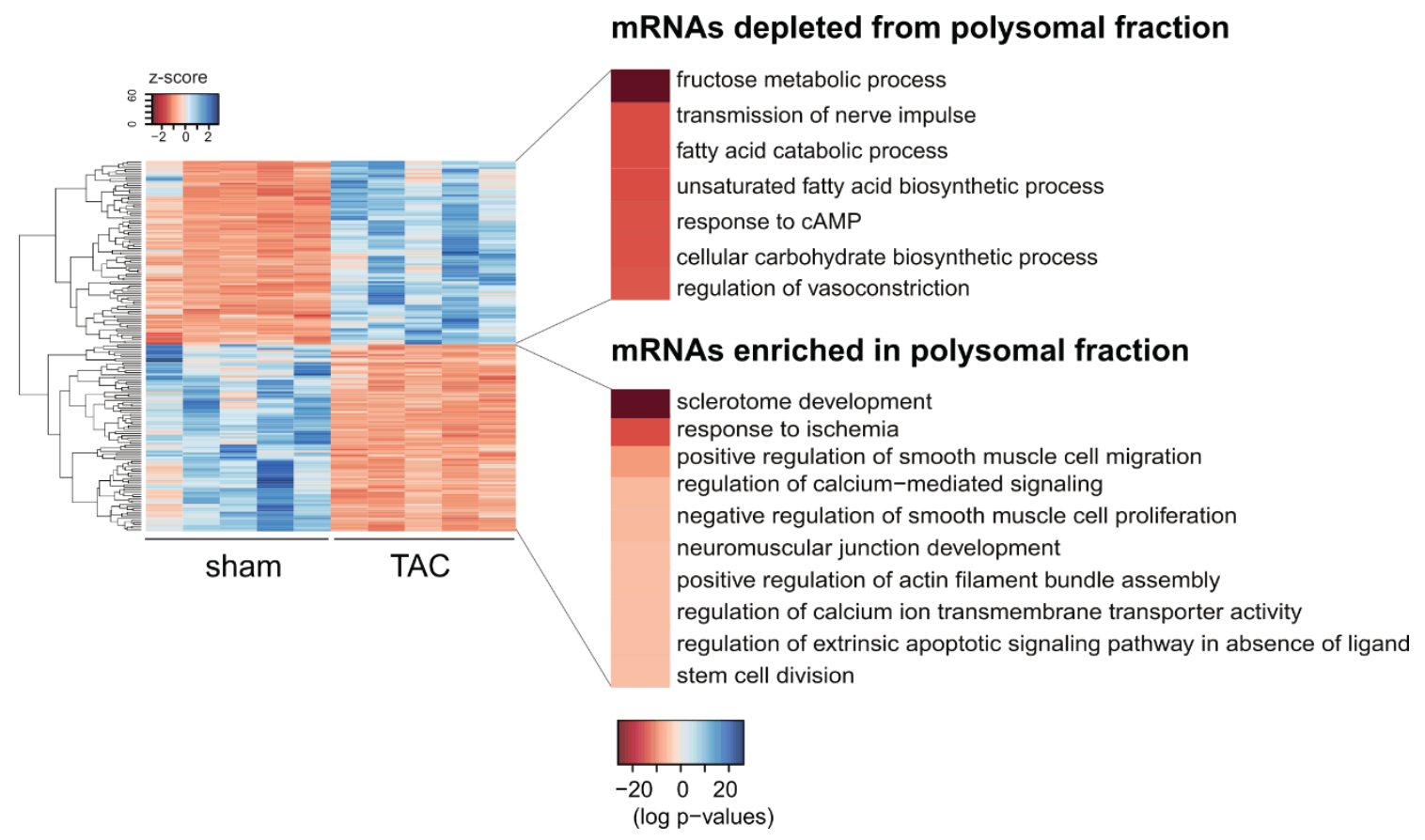

Figure 3.18 The polysomal occupancy of differentially methylated mRNAs is altered upon TAC-induced heart failure in mice

The heatmap shows mRNAs depleted and enriched in polysomal fractions after TAC-induced heart failure in mice. The cut-off for the fold change was set at $\geq 2$ for enriched, and at $\leq 0.5$ for depleted mRNAs with a Padj $\leq 0.05 . \mathrm{n}=5$ for Sham and TAC. Right side: $\mathrm{GO}$ analysis was performed on mRNAs depleted (upper panel) and enriched (lower panel) in cardiac hypertrophy, and GO pathways are presented according to their occurrence in heatmaps. Metabolic and catabolic terms are the most common for depleted mRNAs, and structural pathways accounting for cardiac plasticity as well as signaling are the most common for enriched mRNAs.

\subsubsection{Correlation of methylation and ribosomal occupancy}

Common GO associations identified in hypomethylated transcripts and depleted mRNAs, as well as in hypermethylated pathways and enriched mRNAs, suggest a relationship between methylation changes and translation efficiency. This is further underscored by the fact that identified transcripts are altered at the translation level without being affected at their expression level. Therefore, a correlation analysis between differentially methylated transcripts and differentially translated transcripts was performed (Figure 3.19).

A significant positive correlation was seen between transcripts deregulated at the methylation level and transcripts with altered polysome occupancy $(r=0.37, p=2.2 \mathrm{e}-16)$ in mouse HF 
tissue (8-weeks post-TAC) (Figure 3.19 A). It should be noted, that such correlation was only seen for transcripts with altered methylation in response to cardiac stress or HF, as no correlation was observed in healthy control tissues ( $r=0.003, p=0.8765)$ (Figure 3.19 B). This indicates that a disease-specific mechanism may exist, wherein changes in methylation may directly affect polysomal occupancy, and therefore translation and proteostasis.

A

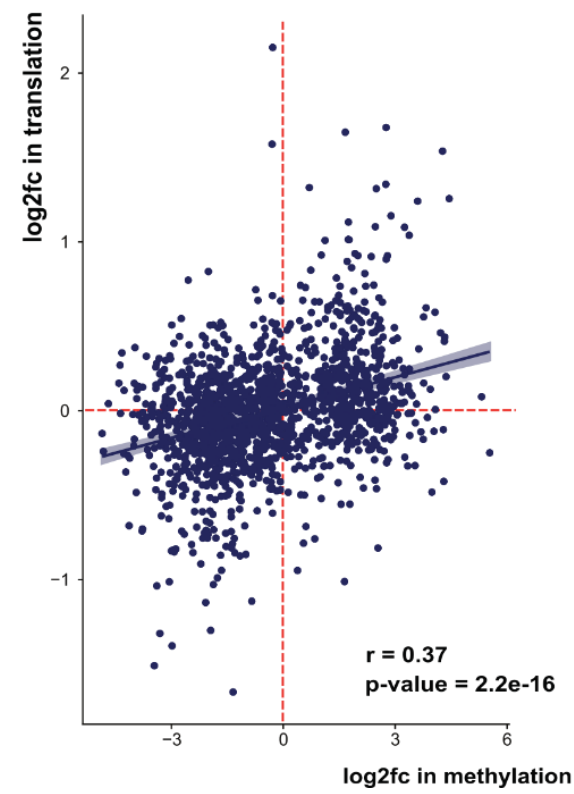

B

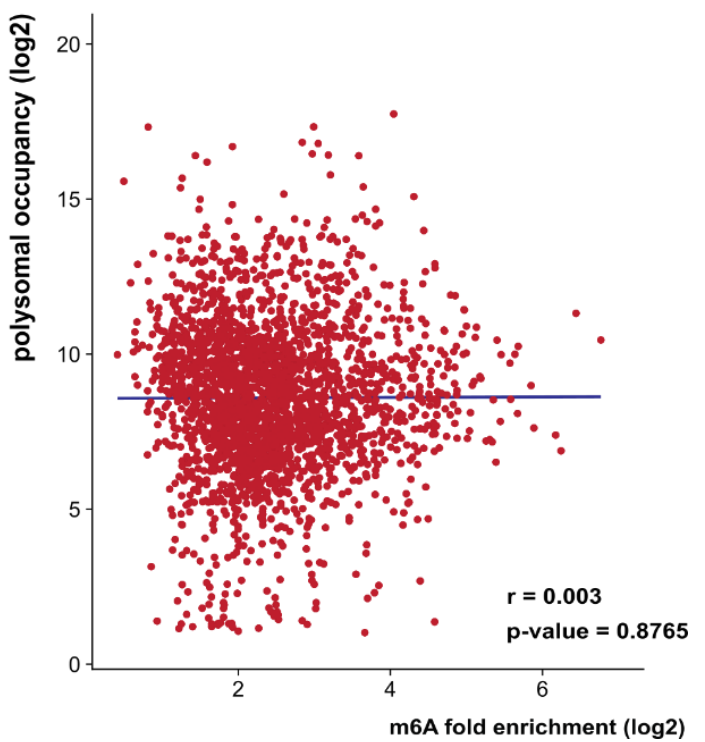

Figure 3.19 RNA methylation correlates with polysomal occupancy and therefore translation in heart failure tissue of mice

(A) Pearson correlation analysis was performed on transcripts from heart failure tissue of mice 8weeks post-TAC which showed altered methylation and altered polysomal occupancy. A positive correlation was found with $r=0.37, p=2.2 \mathrm{e}-16$. (B) The same correlation analysis was performed using data obtained from the healthy heart tissue of mice. Here, no correlation is seen with $r=0.003$, $p=0.8765$.

Polysome analysis was performed only with mouse HF tissue due to sample limitations of available human tissue. To get an idea of the situation in human HF, genes from the correlation analysis in mouse data that have human orthologs were analyzed separately. The methylation level of orthologous human transcripts from human HF tissue was correlated with the differentially translated transcripts from mouse HF tissue (Figure 3.20 A). A strong positive correlation is seen for these orthologous human genes between the methylation and translation level $(r=0.27, p=2.88 \mathrm{e}-08)$.

GO term analysis of transcripts correlating in their methylation and translation level in mouse HF with human orthologs (Figure 3.20 B) suggests that they show a strong cardiac specificity, as represented by a network of GO terms like 'cardiac muscle tissue development', 'muscle system process', and 'vasculogenesis' or 'vasodilatation', as well as metabolic networks like 
'generation of precursor metabolites and energy', and 'fatty acid beta-oxidation using acyl-CoA dehydrogenase'.

These findings may indicate a potential effect of altered methylation on translation and therefore proteostasis.
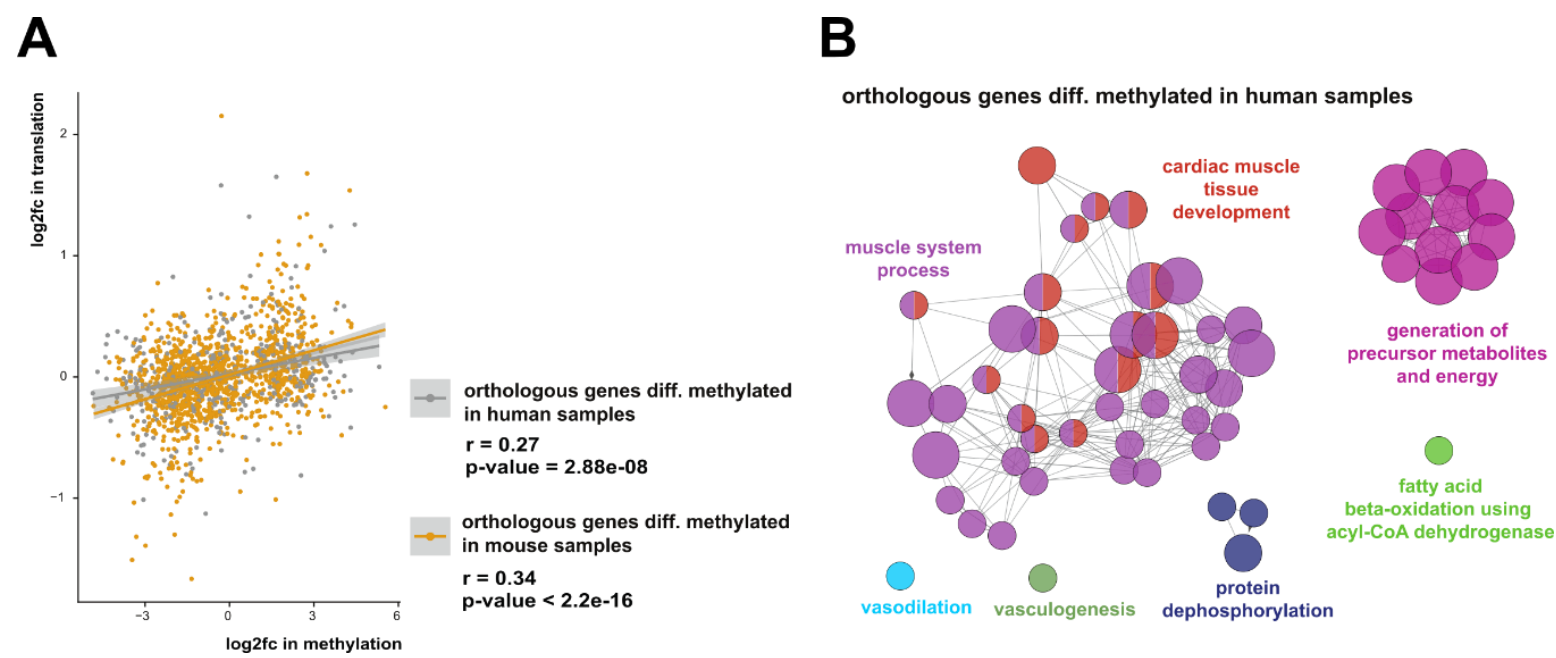

Figure 3.20 Orthologous genes in human samples are of cardiac specificity

(A) An orthologous gene analysis was performed with mouse genes that showed a correlation between methylation level and polysome occupancy. Correlation analysis was performed on genes with human orthologs. Differentially altered methylation levels of genes from human heart failure were correlated with differentially altered translation levels detected by polysomal analysis of mouse heart failure tissue and a correlation is observed with $r=0.27, p=2.88 \mathrm{e}$. A separate correlation analysis was calculated for the same genes with mouse heart failure methylation data and a correlation is seen with $r=0.34, p<2.2 e-16$. (B) GO network analysis was performed for orthologous genes and cardiospecific GO terms were found for those transcripts.

\subsubsection{A potential transcription-independent translation mechanism}

The effect of $\mathrm{m} 6 \mathrm{~A}$ methylation on translation control was already hypothesized by other studies (Li A et al. 2017; Shi $\mathrm{H}$ et al. 2017). In my study, transcripts with altered m6A methylation outnumbered transcripts with changed expression levels by far in response to HT and HF (Figure 3.10 B, Figure 3.12 B, Figure 3.14 B). To further investigate the role of m6A methylation independent of its effect on transcription, transcripts which were identified to be differentially methylated but not differentially expressed were further investigated. Transcripts matching these criteria, and that were further found differentially translated in the polysomes, were further investigated as targets. One cardiac-specific target was identified, namely Calmodulin 1 (CALM1). CALM1 is a calcium ion sensor, activating signal transduction cascades in response to $\mathrm{Ca}^{2+}$, also participating in the $\mathrm{Ca}^{2+} /$ calmodulin-dependent protein kinase II (CamKII) pathway which is important for contraction-coupling in hearts (Boczek et al. 2016). 
Calm1 had unaltered expression levels in HF tissue compared to healthy control tissue but was differentially hypomethylated in both mouse and human heart failure. Furthermore, it showed depletion from polysomes.

The hypomethylation identified by MeRIP, was verified by RT-qPCR (Figure 3.21 B, Figure 3.22 B). Calm1 is decreased in MeRIP fractions of HF tissue compared to healthy control tissue (Figure 3.21 B), consistent with the hypomethylation detected in the sequencing data (Figure 3.21 A). Its depletion from polysomes, and therefore lower translation level was detected by assaying the respective protein level via Western blotting, which demonstrated that the protein level of CALM1 is significantly decreased in HF tissue of mice ( $p=0.03$; Figure 3.21 B right panel).

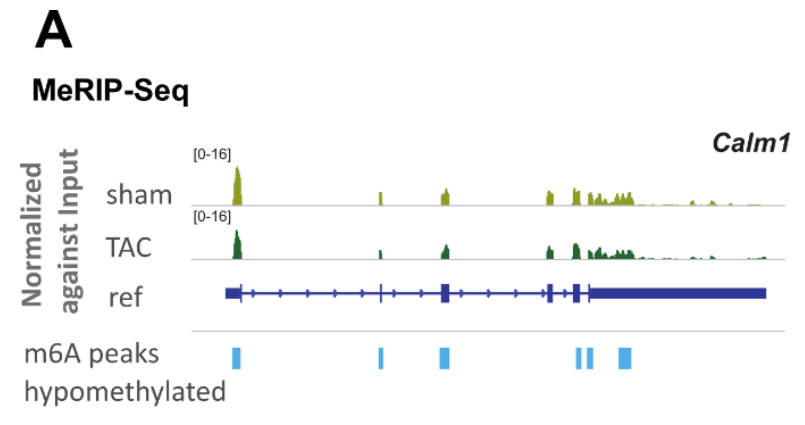

B expression levels m6A level protein level
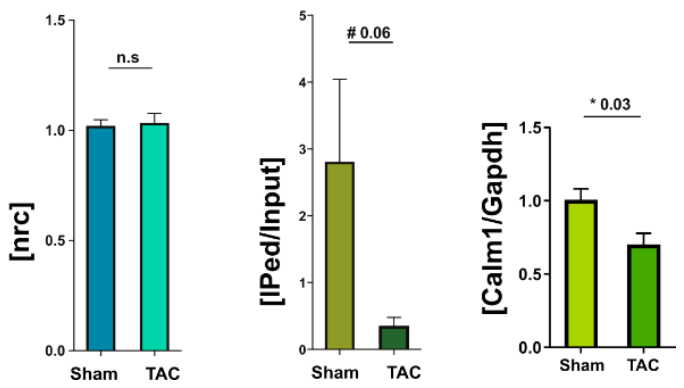

Figure 3.21 CALM1 protein level is decreased despite unaltered expression level in mouse heart failure tissue

Sequencing data from mouse heart failure tissue was screened for genes which are unchanged at the expression level while differentially methylated and found to be deregulated in the polysomes. Calmodulin 1 (Calm1) was identified fulfilling these prerequisites and furthermore being of importance in cardiac tissues. (A) Graphical representation of methylation changes of Calm1 in mouse heart failure tissue found in MeRIP samples compared to respective input. (B) Left panel: the expression level of Calm1 is unchanged in mouse HF with comparable normalized read counts (nrc) obtained from sequencing data of TAC and Sham animals. Middle panel: The hypomethylation of Calm1 as obtained with MeRIP-seq was verified with RT-qPCR. RNA isolated from LV tissue of mice was subjected to MeRIP. Immunoprecipitated (Iped) fractions were normalized to total RNA (Input). A reduced level of transcript was found in TAC tissue in comparison to Sham tissue (Sham: $n=4$, TAC: $n=5$ ). Right panel: Western blotting was performed, and blots analyzed for the CALM1 protein level to verify the change in polysomal occupancy and therefore translation to see if the effect is seen on the protein level. CALM1 bands were normalized to GAPDH and protein level changes determined by normalization to Sham. A reduction of CALM1 protein level $(p=0.03)$ was seen after TAC, correlating with the methylation level reduction (Sham: $n=4$, TAC: $n=8$ ). Full blots are shown in Appendix Figure 5.2. The data is presented as a mean and the SEM indicated. Unpaired t-test was performed, and $p$-values are indicated above the respective bars.

CALM1 was also investigated in human HF tissue (Figure 3.22) to elucidate if a similar effect of m6A methylation on translational control as observed in mouse tissue is present as well. CALM1 shows only a mild decrease of the expression level $(-0.13 \pm 0.06, p=0.06)$ (Figure 
3.22 B left panel) and was hypomethylated as demonstrated by significantly decreased abundance in MeRIP fractions $(-1.0 \pm 0.4, p=0.05$ ) validated by RT-qPCR (Figure $3.22 \mathrm{~B}$ middle panel). Protein level analysis via Western blot revealed a significantly decreased level of CALM1 ( $p=0.04$; Figure 3.22 B right panel) in human HF tissue compared to NF control tissue.

A

MeRIP-Seq

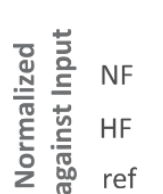

m6A peaks

hypomethylated

\section{B}

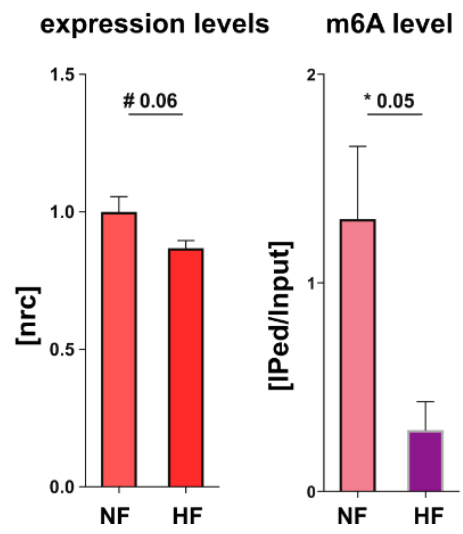

protein level

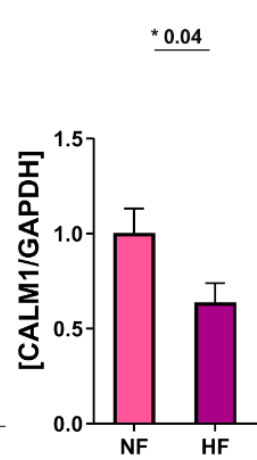

Figure 3.22 CALM1 is hypomethylated and its protein level decreases in human heart failure tissue, despite being unaltered at the expression level.

(A) Graphical representation of the hypomethylation of CALM1 in human end-stage heart failure (HF) in comparison to non-failing, healthy (NF) tissue. (B) Left panel: The transcript abundance is not changed in heart failure tissue (NF: $n=5, H F: n=6$ ) in NGS data. Middle panel: RT-qPCR of samples after MeRIP show a significant reduction of transcripts $(p=0.05)$ indicating hypomethylation (NF: $\mathrm{n}=$ $3, \mathrm{HF}: \mathrm{n}=4$ ). Right panel: The protein level shows a significant decrease of CALM1 in HF as analyzed with Western blot (NF: $n=3 \mathrm{HF}: \mathrm{n}=5)$. Respective Western blots are shown in Appendix Figure 5.2. Data is represented as a mean and with the SEM indicated. Unpaired t-test was performed, and $p$ values are indicated above the respective bars.

Since the CALM1 protein level significantly decreased in both mouse and human LV tissue, a potential new mechanism of transcription independent translational control mediated by $\mathrm{m} 6 \mathrm{~A}$ RNA methylation was proposed. 


\subsection{Manipulation of the methylation machinery in iPSC-CMs}

NGS and MeRIP-seq of LV tissues in my study demonstrated that the methylation level is considerably changed upon cardiac HT and HF. Since the reversible m6A methylation is mediated by readers and writers it was of further interest to me to elucidate potential effects of a manipulation of the methylation machinery on the hypertrophic stress response. By depletion of the m6A demethylase FTO in vitro and in vivo, potential altered response to hypertrophic stimuli in CMs was investigated.

As CMs are post-mitotic cells, they cannot be easily grown in cell culture and their cultivation as primary cell cultures is rather difficult. Therefore, a human induced pluripotent stem cell (iPSC) model was used. iPSCs are a widely used tool for in vitro studies (Shi Y et al. 2017; House et al. 2020). They are capable of self-renewal and have the ability to differentiate into all three germ layers and the germline (Jaenisch and Young 2008). A well-established protocol of differentiation of iPSCs to beating CMs exists (Lian et al. 2013) and therefore allows an easy in vitro study of the cardiomyocyte model.

\subsubsection{Differentiation of iPSCs to cardiomyocytes}

Two cell lines were used in this study, to verify that observed results are cell line independent. The two cell lines used in this study, pCTRL1.1 (pCTRL) and WT-D2-1U (WTD2), were already characterized for their pluripotent capacity and expression of typical markers (StreckfussBömeke et al. 2013; Borchert et al. 2017) and are widely used. iPSCs were a kind gift from PD Dr. Katrin Streckfuß-Bömecke (Clinic for Cardiology and Pneumology, University Medical Center Göttingen). Both cell lines were reprogrammed from keratinocytes obtained from healthy donor patients and can be considered as wildtypes. A differentiation protocol utilizing activation and inhibition of the canonical Wnt-pathway in a time-dependent manner was used (Lian et al. 2013) to generate iPSC-derived cardiomyocytes (iPSC-CMs) (refer to Methods,

Section 2.3.2). Following observation of spontaneous beating around day 10 after initial induction of differentiation, cells were further metabolically selected to obtain a purer cardiomyocyte population. This was done since not all iPSCs differentiate into CMs. By culturing cells in selection medium containing lactic acid as sole energy source, this leads to survival of mostly CMs which are able to metabolize lactic acid. Surviving CMs were further cultured for at least 60 days after initial induction of differentiation to obtain mature iPSC-CMs with a stable expression of a cardio-specific gene program and morphology (Shinozawa et al. 2012). 
In my doctoral work, I needed to establish a hypertrophic growth model for the mature iPSCCMs as well as establish a protocol for depletion of FTO. Establishing protocols are described in more detail in the following chapters. It needs to be mentioned, that not all experiments for establishing protocols could be performed with both cell lines, as in some differentiation experiments not enough mature iPSC-CMs were obtained. Experiments needed to be planned at least 60 days in advance considering the maturation and during these 60 days some cells were lost due to detachment or contamination. However, later described experiments investigating the effect of the depletion on hypertrophic growth were performed with both cell lines to verify that results are cell line independent.

\subsubsection{Establishing an ET-1-induced hypertrophy model in iPSC-CMs}

To study effects of FTO depletion in response to cardiac stress, a hypertrophic growth/response model for iPSC-CMs was utilized. Hypertrophy can be induced in vitro by stimulating iPSC-CMs with compounds mimicking hypertrophic pathways by transcription factor activation (Moreau et al. 1997; Gray 1998; Adiarto et al. 2012; Bupha-Intr et al. 2012). Endothelin-1 (ET-1) stimulates the renin-angiotensin-aldosterone system, and activates the CaMkll pathway (Moreau et al. 1997), leading to cardiomyocyte hypertrophy. This system was applied to produce hypertrophic cells and further combined with depletion of FTO via siRNA transfection to investigate potentially altered hypertrophic response in vitro.

Different ET-1 concentrations were described in literature (Zlabinger et al. 2019) to induce cellular hypertrophy. To determine a suitable concentration of ET-1 to use in my cell culture, I investigated three different previously described concentrations, namely $1 \mathrm{nM}, 2 \mathrm{nM}$, and $3 \mathrm{nM}$ in each cell line.

Cell growth was monitored via immunofluorescence (IF) microscopy by staining $\alpha$-actinin (Figure 3.23 A, C). a-actinin is a microfilament protein attaching actin filaments to Z-lines in muscle cells (Ribeiro et al. 2014), and is therefore a marker of CMs in cell culture. By measuring the cell sizes of $\alpha$-actinin stained, ET-1-stimulated cells in comparison to nontreated (mock) control cells, the effect of the different ET-1 concentrations was determined (Figure $3.23 \mathrm{~B}, \mathrm{D}$ ).

Hypertrophic growth was observed in both cell lines in all three conditions (Figure 3.23), with $2 \mathrm{nM}$ resulting in largest cell size increase in pCTRL cells ( $p=0.03$ Figure $3.23 \mathbf{B}$ ), whereas 3 nM ET-1 induced a stronger response in the WTD2 cell line as seen by a larger mean cell size ( $p=0.0008$ ) (Figure $3.23 \mathrm{D}$ ). Since $1 \mathrm{nM}$ and $2 \mathrm{nM}$ of ET-1 induced comparable hypertrophic growth in WTD2 cells, but a concentration of $3 \mathrm{nM}$ exerted an even stronger response, this concentration was chosen to be used in following experiments. Although a concentration of 2 
nM ET-1 induced the strongest cell growth in PCTRL cells, a concentration of $3 \mathrm{nM}$ was used with these cells for further experiments to keep experimental conditions similarly. This was decided regarding that 3 nM ET-1 as well induced hypertrophic cell growth in pCTRL cells.

A

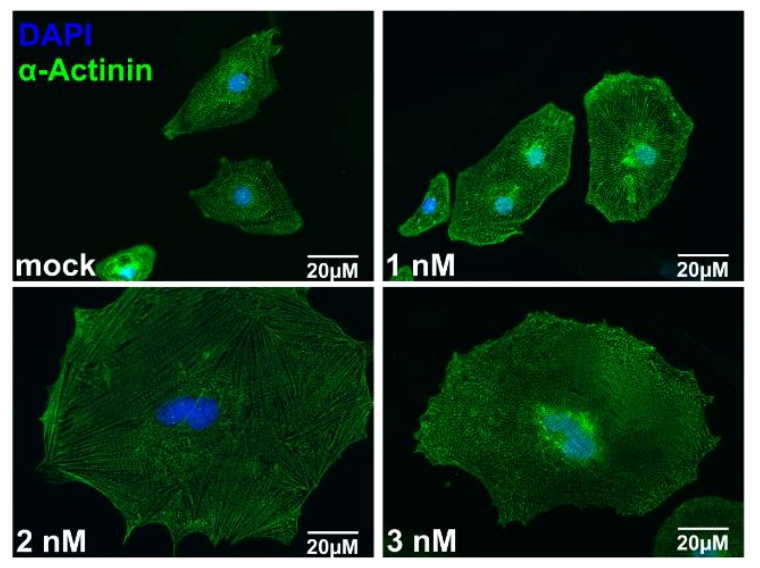

C

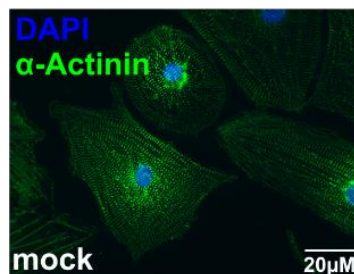

mock $\overline{20 \mu \mathrm{M}}$

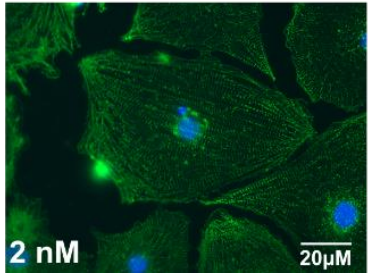

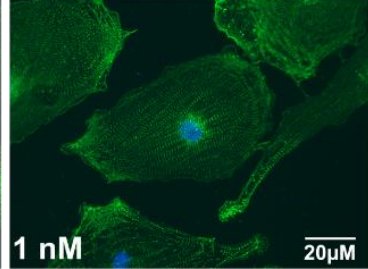

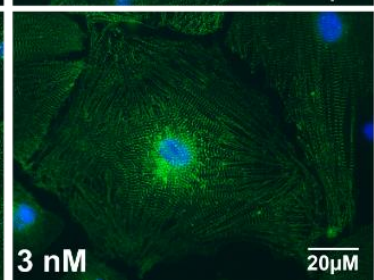

B

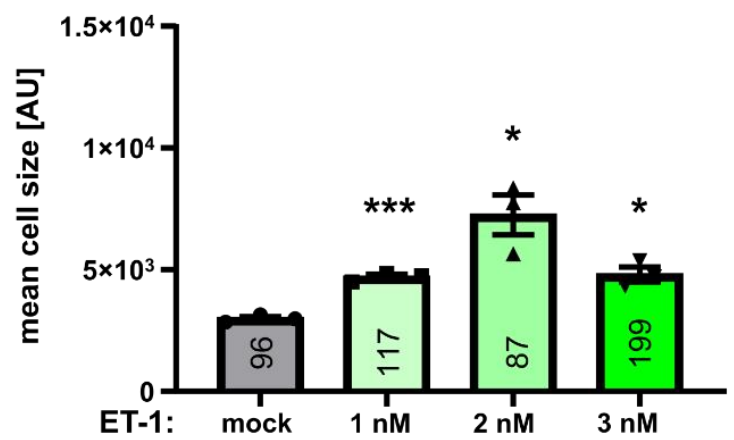

$\mathbf{D}$

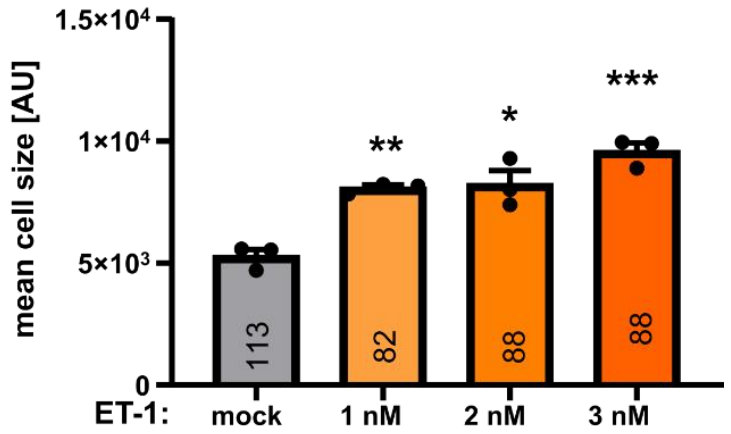

Figure 3.23 Concentration determination of ET-1 treatment

ET-1 was applied at a concentration of $1 \mathrm{nM}, 2 \mathrm{nM}$, and $3 \mathrm{nM}$ to hiPCS-CMs of both cell lines ( $\mathrm{pCTRL}$ and WTD2) to induce hypertrophic responses and growth. Since ET-1 is dissolved in water, cells treated with water served as mock-control. (A) Representative IF images of PCTRL cells are shown. The cells were stained for a-actinin and the nucleus with DAPI. Images were taken using the FITC channel (green) as well as the DAPI channel (blue) at 400X magnification with an oil-immersion objective. (B) Cell sizes of ET-1 stimulated PCTRL cells were determined by measuring the cell area (presented in arbitrary units (AU)) and growth changes calculated. Significant increases of cell size can be seen in all three conditions. Cells from one differentiation experiment were used and three object-slides per condition imaged. The mean of the cell areas measured per slide was taken for statistical analysis. t-test with Welch's correction was performed and significance levels are given per condition in comparison to the mock control. The mean with SEM is presented and the $n$ of all counted cells per condition on all slides together is given in the respective bars. (C) Representative IF images of mock and ET-1-stimulated WTD2 cells stained for $\alpha$-actinin and DAPI are shown. (B) The cell sizes of WTD2 cells after ET-1-stimulation were measured and a significant increase of the cell size can be seen for all three conditions. Experimental design and data analysis were equally as with PCTRL cells. 


\subsubsection{Establishing siRNA- mediated silencing of FTO in iPSC-CMs}

Acting as an m6A RNA methylation eraser, the demethylase FTO is a key player in the dynamic and reversible modification of m6A marks (Gerken et al. 2007; Jia et al. 2011). When FTO levels are decreased, less demethylation activity is occurring and therefore RNA methylation should increase, leading to a global hyper-methylation (Jia et al. 2011). By this manipulation of the epitranscriptome, the effect on the hypertrophic responses of CMs may be addressed. In the iPSC-CM model, siRNA-mediated gene silencing was chosen to decrease the FTO activity and therefore induce global mRNA hypermethylation. siRNAs act as silencing agents by binding complementary mRNA targets via the RNAi pathway inhibiting translation or leading to mRNA degradation (Carthew and Sontheimer 2009; Zhang 2013). siRNA mediated knockdown was the preferred method for my study, since genetically engineering a stable knockout in cell culture, e.g. by CRIPR/Cas is time consuming and may induce off-target effects and iPSCs may lose the ability to differentiate to for example CMs when genetically modified (Chaterji et al. 2017). Furthermore, epigenetic factors can impair the differentiation potential of iPSC-CMs (Keller et al. 2018). Since FTO silencing should affect RNA methylation and hence epigenetic modifications, a residential KO may impair differentiation ability. siRNAs can be applied to already differentiated iPSC-CMs, therefore this method was chosen to achieve FTO depletion.

In preliminary experiments, four different siRNAs which all bind to different regions in the FTOmRNA were transfected for 24 hours to determine the most effective one for FTO depletion (Appendix Figure 5.3). The best performing siRNA was further tested with a longer time period of 48 hours transfection to potentially strengthen the silencing effect. An overview of the knockdown-protocol is presented in Figure 3.24.

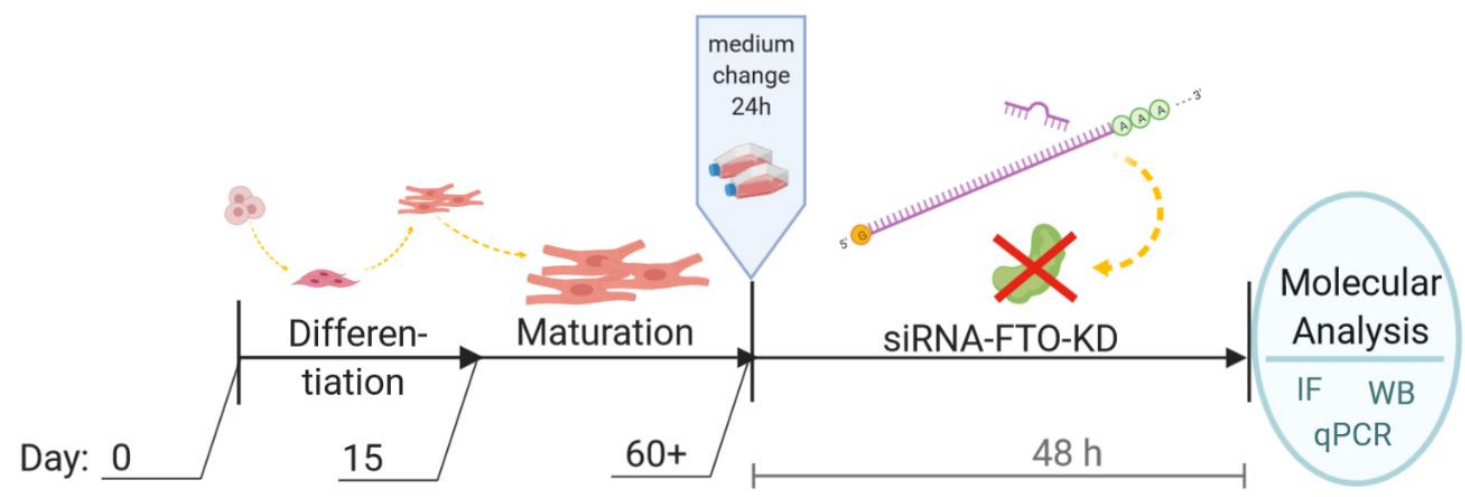

Figure 3.24 Initial transfection protocol

iPSC derived CMs were matured for at least 60 days $(60+)$ after initial start of differentiation (Day 0$)$. Fresh medium was added to the cells 24 hours prior to the transfection, and cells were transfected with FTO-targeting siRNA for 48 hours (48 h). Afterwards, cells were harvested for further molecular analysis. 
Effective silencing of FTO in iPSC-CMs by the siRNA of interest was validated the mRNA level by RT-qPCR and on the protein level via Western blotting after a transfection time of 48 hours. A scrambled RNA without a complementary sequence in the human genome served as a negative control to rule out transfection-related side effects. Cells with silenced FTO mediated by the siRNA are called FTOsikD in the following experiments, while respective negative controls treated with the scrambled siRNA are further called FTO ${ }^{\text {WT }}$.

A significant reduction of FTO-mRNA by $36 \%(p=0.03)$ in pCTRL FTO ${ }^{\text {sikD }}$ cells (Figure 3.25 $\mathbf{A}$,) and by $78 \%(p=0.0003)$ in WTD2 FTO ${ }^{\text {sikD }}$ cells (Figure $3.25 \mathbf{B}$ ) was seen in comparison to the FTO ${ }^{W T}$ cells of both cell lines (Figure 3.25 A, B). Furthermore, Western blotting of the FTO protein level in WTD2 cells demonstrated significantly decreased FTO protein level in the WT cell line by $58 \%$ in $\mathrm{FTO}^{\text {sikD }}$ cells compared to $\mathrm{FTO}^{\mathrm{WT}}(p=0.03$ ) (Figure $3.25 \mathrm{D}$ ). Western blotting could not be performed with $\mathrm{PCTRL}$ cells due to sample limitations caused by technical difficulties during maturation. 


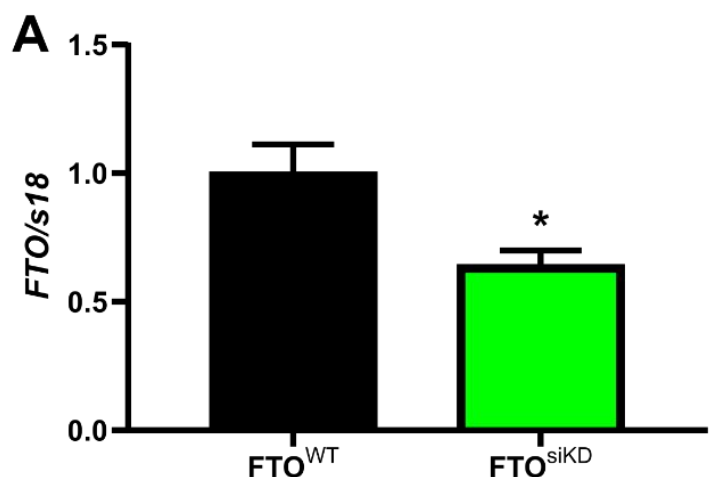

C

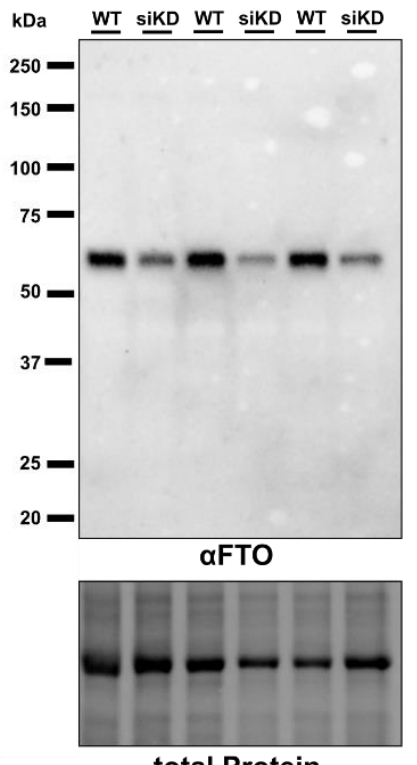

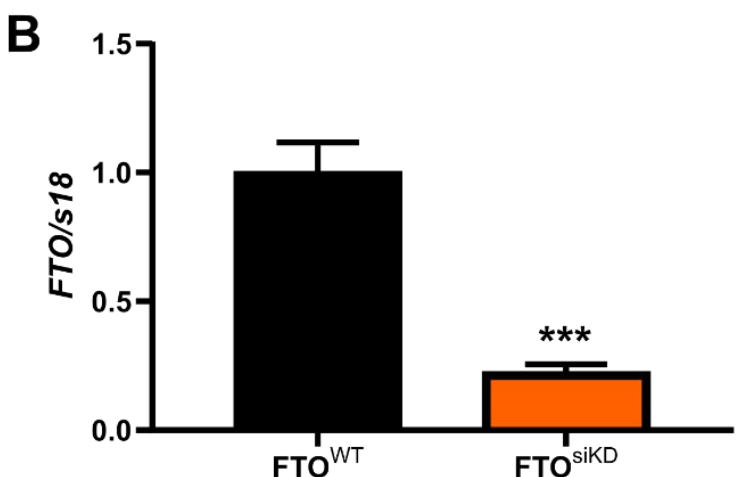

D

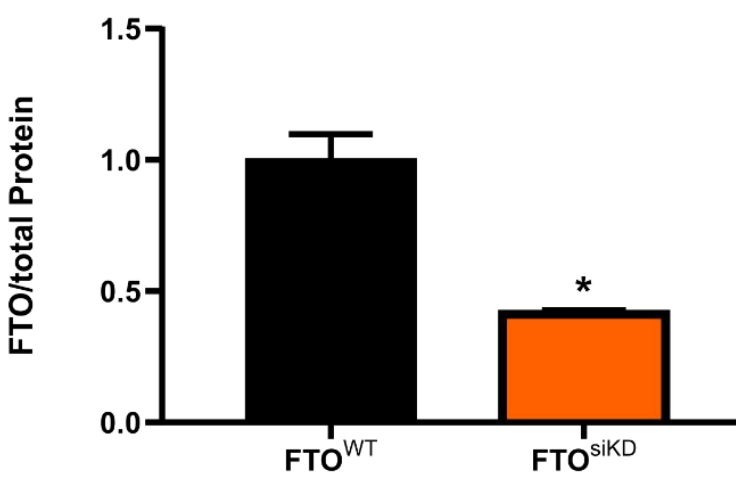

Figure 3.25 A stable FTO knockdown on the mRNA level was induced 48 hours after transfection

iPSC-CMs of the pCTRL and WTD2 cell line were transfected for 48 hours with an FTO-targeting siRNA. (A) RT-qPCR revealed a significant decrease of FTO-mRNA in siRNA transfected cells (FTOsikD) of the pCTRL cell line ( $n=5$ for FTOWT and FTO sikD) (B) as well as the WTD2 cell line $(n=$ 6 for for FTOWT and $\mathrm{n}=4$ for FTOsikD). RT-qPCR cycles of FTO were normalized against the s18 housekeeping gene and changes in RNA level were normalized to scrambled siRNA treated controls (FTOWT) with the ddCt method and the mean with SEM is presented. t-test with Welch's correction was for statistical analysis. (C) Western blotting of WTD2 protein lysates shows specific protein bands for FTO (58 kDa, upper panel). A representative part of the respective total protein lanes is shown (lower panel). Each lane represents an independent biological replicate of each group (WT = FTOWT, $n=3$; siKD $=$ FTOsiKD,$n=3$ ). (D) FTO protein level is significantly decreased after 48 hours of siRNA transfection. The FTO protein bands were quantified and normalized to the total protein. Changes in protein level in siRNA treated cells (FTO ${ }^{\text {sikD }}$ ) was calculated in relation to scramble treated control cells (FTOWT) and statistically tested with t-test with Welch's correction. The mean with SEM is presented. Cells from one differentiation experiment were used, with different wells as biological replicates ( $n=3$ for FTOWT and FTO ${ }^{\text {sikD }}$ ). 


\subsubsection{Establishing a combined siRNA-transfection and ET-1 stimulation protocol}

After having determined a suitable transfection time of 48 hours for siRNA-mediated silencing of FTO it was of interest to combine this siRNA transfection protocol with the ET-1-induced hypertrophy protocol (Figure 3.26).

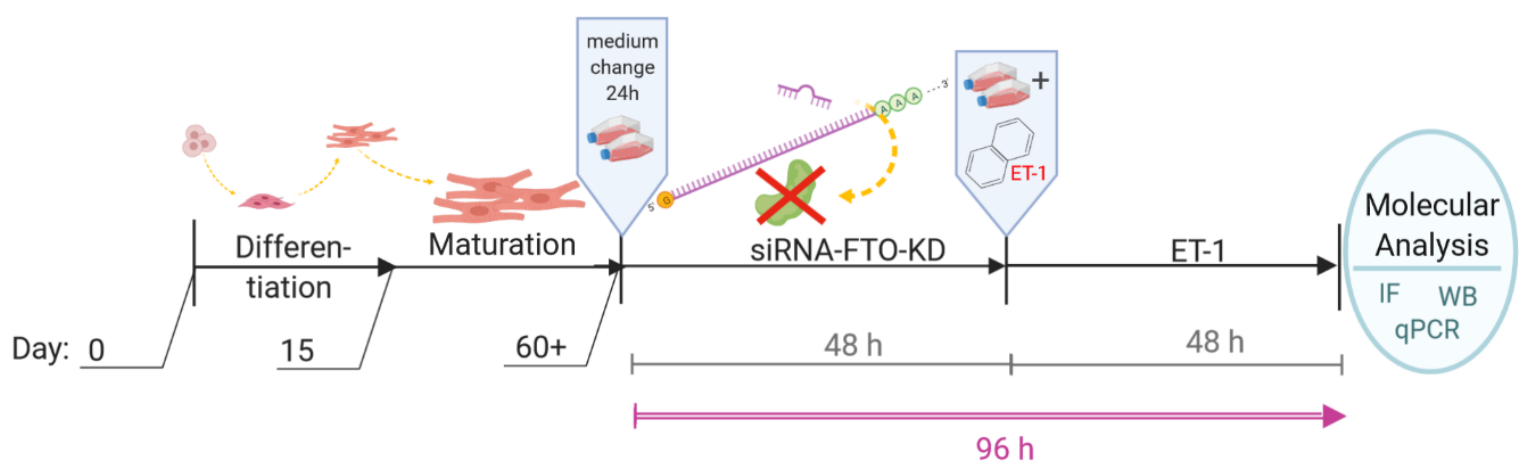

Figure 3.26 Combined siRNA-transfection and ET-1 treatment protocol

iPSC derived CMs were matured at least 60 days $(60+)$ after initial differentiation induction (day 0$)$. Fresh medium was added to the cells 24 hours prior to the transfection, and cells were transfected with siRNA for 48 hours. The medium was changed, and ET-1 was added at a concentration of $3 \mathrm{nM}$ for 48 hours. Afterwards, cells were harvested for further molecular analysis.

Since ET-1 stimulation takes place in absence of siRNAs it was first necessary to validate that the knockdown would still be effective during ET-1 stimulation time until the end of treatment time. Therefore, iPSC-CMs of the WTD2 cell line were transfected with FTO-siRNA for 48 hours, medium changed to fresh medium without siRNAs but supplemented with ET-1 at $3 \mathrm{nM}$ concentration, and the FTO-mRNA as well as protein level analyzed after 48 hours of ET-1 stimulation. This results in a time period of 96 hours from the transfection to the final cell harvest for molecular analysis.

On the mRNA level, the silencing effect is still measurable 48 hours after removal of siRNA containing medium (Figure 3.27 A). FTO-mRNA is significantly decreased in FTO ${ }^{\text {siKD }}$ cells treated with 3 nM ET-1 $(p=0.04)$. In mock-treated FTO ${ }^{\text {sikD }}$ cells the FTO-mRNA level is not significantly decreased $(p=0.07)$. This lower decrease of the mRNA level may derive from newly transcribed mRNA, not silenced after the siRNA was removed.

On the protein level, analyzed with Western blot (Figure 3.27 B), the FTO protein is significantly decreased after 48 hours of ET-1 stimulation or mock control treatment ( $p=0.01$ for both 
conditions) in $\mathrm{FTO}^{\text {siKD }}$ cells compared to $\mathrm{FTO}^{\mathrm{WT}}$ (Figure 3.27 C). Comparable experiments were not performed on PCTRL cell line due to technical difficulties as described before.

A

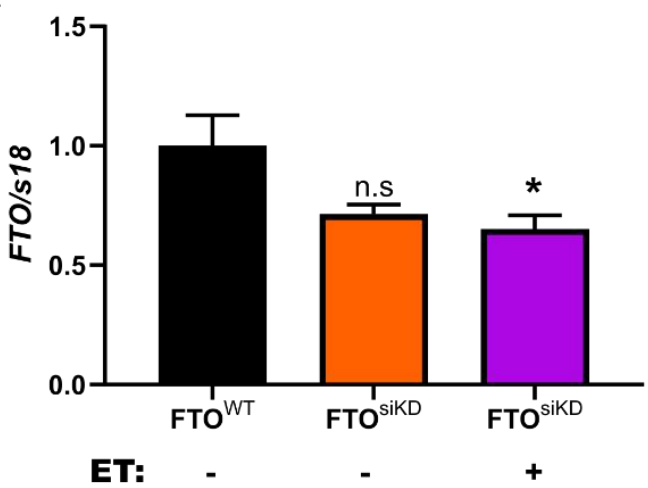

B

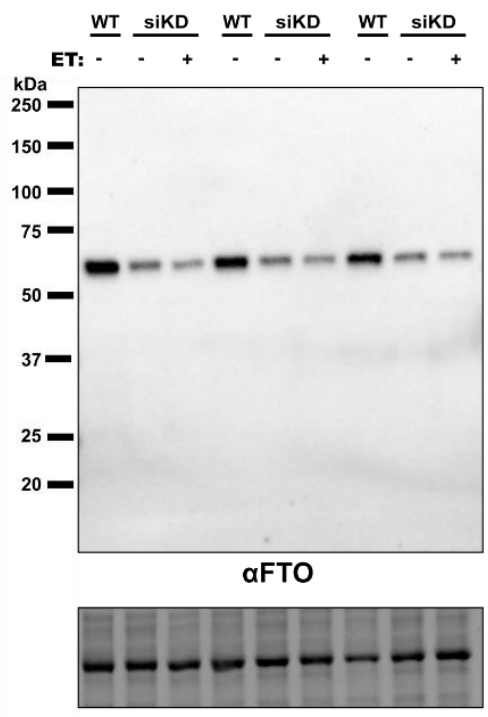

C

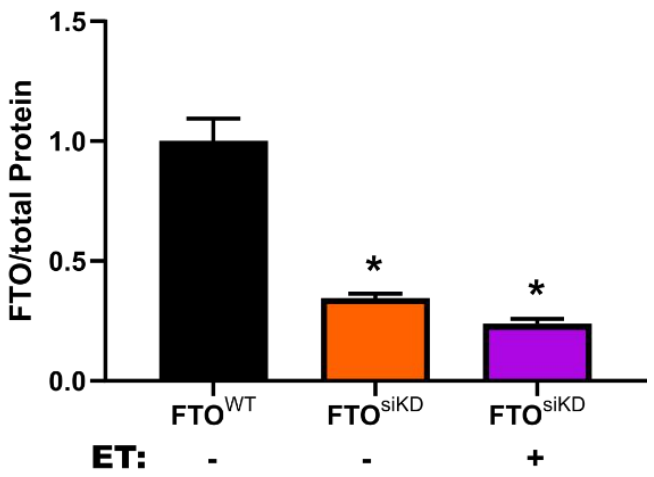

Figure 3.27 The Fto-KD is still active 48 hours after siRNA removal

iPSC-CMs of the WTD2 cell line were transfected with siRNA targeting FTO for 48 hours (FTOsikD) or scrambled siRNA as control (FTOWT), siRNA transfection medium removed, and cells further treated with $3 \mathrm{nM} \mathrm{ET-1} \mathrm{(ET:} \mathrm{+).} \mathrm{Water} \mathrm{served} \mathrm{as} \mathrm{mock} \mathrm{control} \mathrm{(ET:} \mathrm{-)} \mathrm{(A)} \mathrm{The} \mathrm{FTO-KD} \mathrm{was} \mathrm{evaluated} \mathrm{on} \mathrm{the}$ mRNA level with RT-qPCR using the ddCt method. RT-qPCR cycles of FTO were normalized against the $s 18$ housekeeping gene, and the results were normalized to the mock treated cells with scrambled siRNA transfected (FTOWT). Respective significance levels were obtained from a t-test with Welch's correction. Different wells, treated equally, from one differentiation experiment served as biological replicates with $\mathrm{n}=6$ per group and each sample was pipetted in duplicates for technical replication. (B) Western blot images shows specific protein bands for FTO (58 kDa, upper panel). A representative part of the respective total protein lanes is shown (lower panel). A visible reduction of FTO level can be seen in both, mock and ET-1 treated FTOsikD cells in comparison to mock treated FTOWT cells. Each lane represents an independent biological replicate of each group (WT = FTOWT; siKD = FTO ${ }^{\mathrm{siKD}}$ ) (C) The FTO protein bands were quantified and normalized against the corresponding total protein lane. Protein level changes were calculated in comparison to mock treated FTOWT cells. $\mathrm{n}=3$ per group. The mean with SEM is presented. T-test with Welch's correction was performed to determine significance levels. 
Although if the silencing effect described in Section 3.2.4 was measurably significant on the FTO protein level the transfection protocol was optimized to strengthen the silencing effect and possibly decrease the FTO protein level even more after ET-1 stimulation. This was done by adding a second siRNA transfection step 24 hours after initial transfection (Figure 3.28). This shortens the time period from the last transfection to the final cell harvest for molecular analysis after ET-1 stimulation from 96 hours to 72 hours.

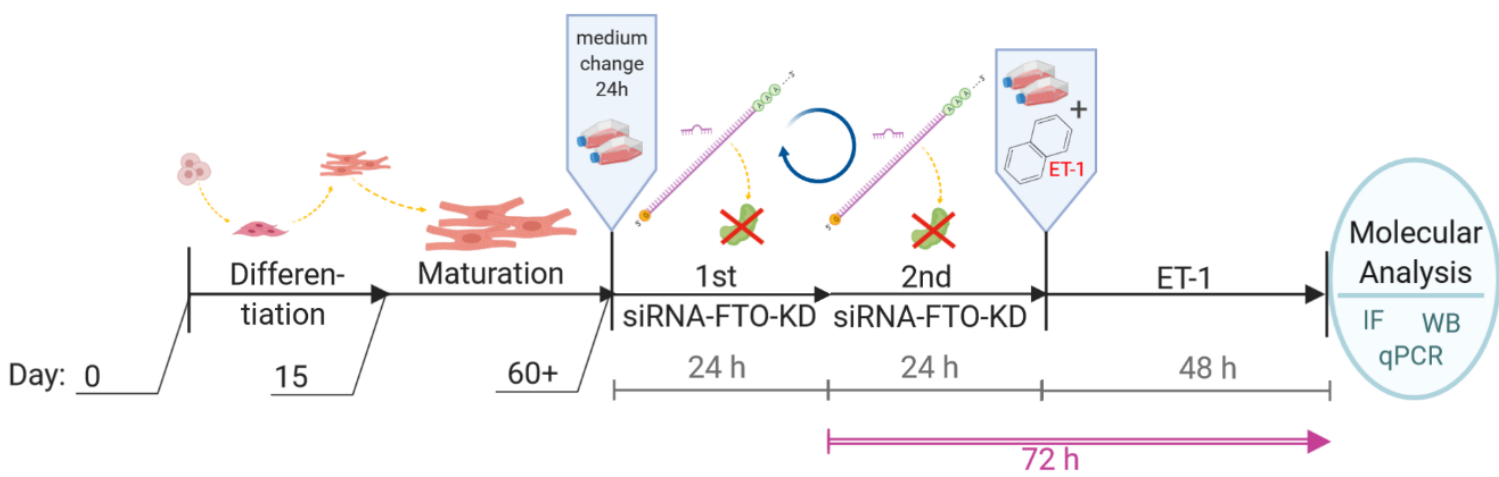

Figure 3.28 Adapted siRNA transfection protocol combined with ET-1 stimulation

The initial siRNA transfection protocol was adapted to obtain a stronger and more persistent knockdown during the ET-1 treatment period. To achieve this, an additional transfection step was added to shorten the time from the last siRNA transfection to harvesting of cells after $3 \mathrm{nM} E T-1$ treatment from 96 hours to 72 hours in total.

To validate that the adapted protocol results in a stable decrease of the FTO protein level, iPSC-CMs of both pCTRL and WTD2 cell line were transfected with siRNA for 24 hours twice. After the second transfection step, medium was changed to medium without siRNA and cells harvested after additional 48 hours incubation. In order to spare cells for other experiments, since maturation is laborious and error-prone as described before, for this establishing experiment, only the mock condition was tested without the ET-1 group. Since comparable protein levels were seen in FTO ${ }^{\text {sikD }}$ cells treated with either mock or ET-1 in the previously described experiment (Figure 3.27), it was assumed that for this experiment similar results as seen with mock-treated cells would be obtained if cells were treated with ET-1.

Western blot analysis of the protein level of FTO reveals, that this adapted protocol induces a stronger, significant decrease of FTO in both cell lines in FTO ${ }^{\text {sikD }}$ cells compared to FTOWT cells (pCTRL: $50 \%, p<0.0001$; WTD2: 85\%; $p=0.0003$ ) (Figure 3.29 B, D). Since the protein level accounts for the cellular action of FTO, the adapted protocol was considered suitable for further experiments and the mRNA level was not analyzed. 
A
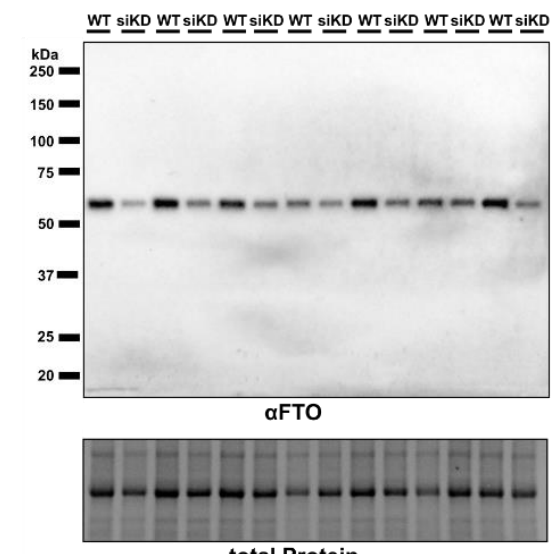

C

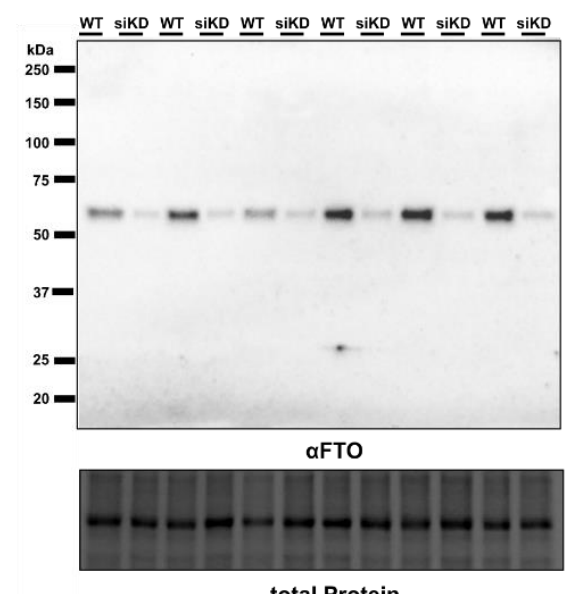

B

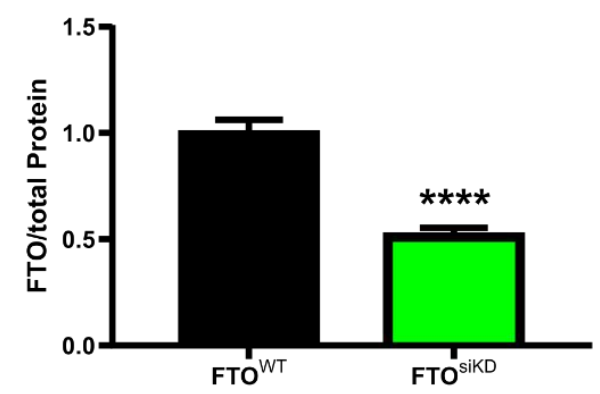

D

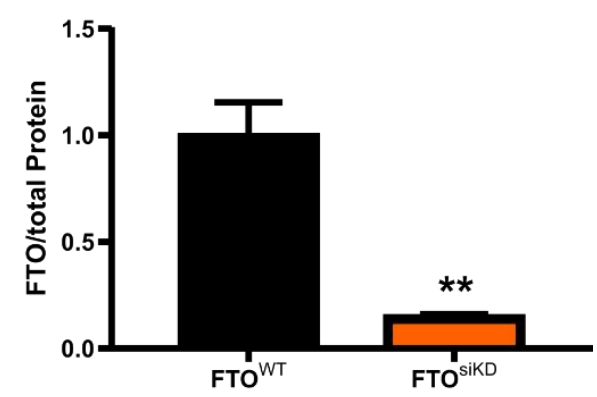

Figure 3.29 The FTO protein level is significantly decreased following the adapted protocol The FTO protein level was significantly decreased when siRNA is transfected according to the adapted protocol. (A) Western blot image of FTO protein in PCTRL cells. A clear band at $58 \mathrm{kDa}$ was obtained. Respective total protein lanes used for normalization are presented. Each lane represents an independent biological replicate of each group (WT $=\mathrm{FTO}^{\mathrm{WT}}, \mathrm{n}=7$; siKD $=\mathrm{FTO}^{\text {siKD }}, \mathrm{n}=7$ ). (B) FTO protein bands were quantified and normalized against total protein in PCTRL cells. The protein level change is calculated by comparing FTO ${ }^{\text {sikD }}$ to FTOWT (C) Western blot images and corresponding total protein lanes for the WTD2 cell line is presented. Each lane represents an independent biological replicate of each group (WT $=$ FTOWT $^{W}, \mathrm{n}=6$; siKD $=$ FTO ${ }^{\text {sikD }}, \mathrm{n}=6$ ) (D) Quantification of the FTO protein in WTD2 cell lines demonstrates a significant reduction of FTO in FTOsikD cell compared to FTOWT cells. The mean with SEM is presented. t-test with Welch's correction was used and significance levels shown on the bars. Different wells, treated equally, from one differentiation experiment served as biological replicates. $n=7$ for FTOWT and FTO ${ }^{\text {sikD }}$ of the $P C T R L$, $\mathrm{n}=6$ for FTOWT and FTO ${ }^{\text {sikD }}$ of the WTD2 cell line. 


\subsubsection{The effect of silencing FTO on ET-1 induced hypertrophic growth}

Having established a working protocol, with which stable silencing of FTO followed by ET-1 stimulation can be induced, it was then of interest to investigate the effects of this silencing on the hypertrophic response of iPSC-CMs.

\subsubsection{Silencing of FTO attenuates cell growth}

To assess the effect of FTO knockdown on the hypertrophic response of iPSC-CMs, cell size measurements were performed by means of immunofluorescence microscopy (Figure 3.30) with experimental setup similar to the initial ET-1 concentration determination assay (Section 3.2.2).

Stimulation of cells after siRNA mediated FTO manipulation with ET-1 following the established protocol (Figure 3.28) induces a significant hypertrophic growth in scrambled siRNA transfected FTO ${ }^{W T}$ cells of the pCTRL cell line $(p=0.004$ Figure $3.30 \mathrm{~B})$. A slight growth can be appreciated $(p=0.08)$ in FTOWT cells of the WTD2 cell line (Figure $3.30 \mathrm{D})$. Upon silencing of FTO with siRNA, hypertrophic growth was completely attenuated in FTO ${ }^{\text {sikD }}$ cells of the $P C T R L$ cell line ( $p=0.9)$ when stimulated with ET-1, since the cell size did not increase compared to the respective mock treated $\mathrm{FTO}^{\text {sikD }}$ cells (Figure 3.30 B).

In WTD2 cells, a trend toward hypertrophic growth may be recognized in FTO ${ }^{\text {sikD }}$ cells upon ET-1 stimulation compared to FTO ${ }^{\text {sikD }}$ mock treated cells $(p=0.2)$, but to a lesser extent than in the $\mathrm{FTO}^{\mathrm{WT}}$ group $(p=0.07$ ) (Figure $3.30 \mathrm{D}$ ).

When comparing sizes of ET-1 stimulated cells it is apparent, that cells bearing the FTO knockdown (FTO ${ }^{\text {sikD }}$ ) are smaller in size compared to the FTO ${ }^{W T}$ group in both cell lines (pCTRL $p=0.01$, Figure 3.30 B; WTD2 $p=0.07$, Figure $3.30 \mathrm{D}$ ), indicating an attenuated growth related to the silencing of $F T O$. 
A
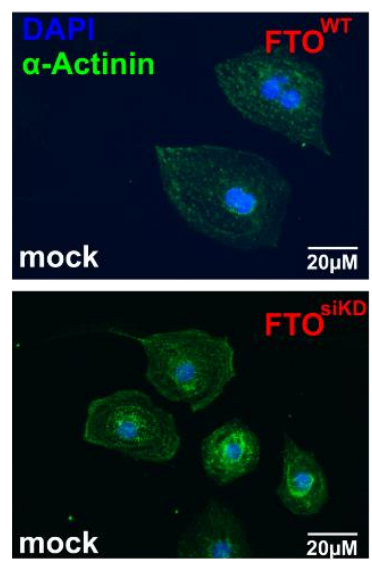
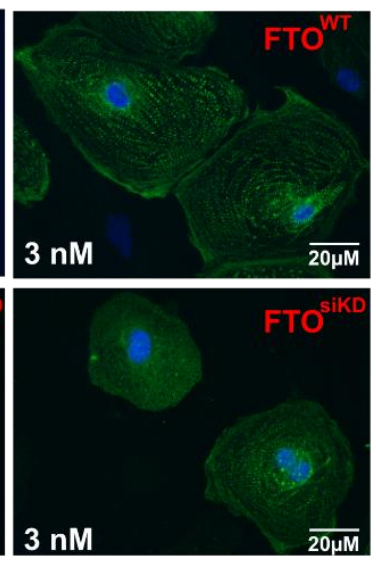

B

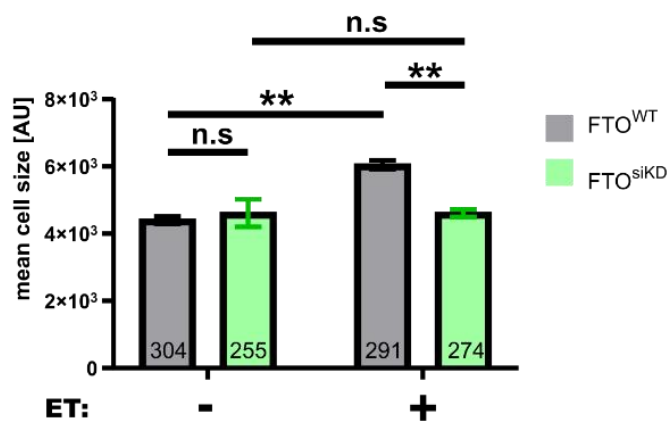

C

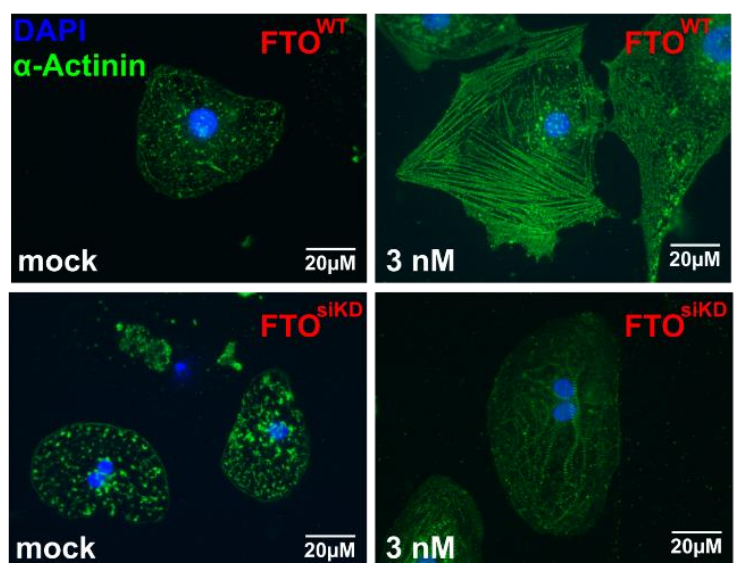

D

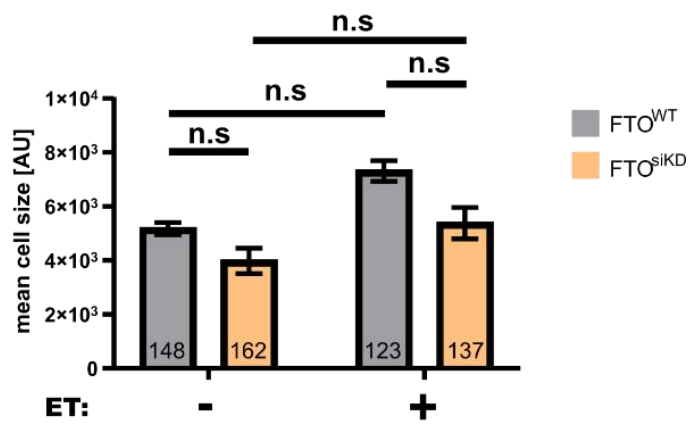

Figure 3.30 FTO-KD attenuates cell growth

ET-1 was applied at a concentration of $3 \mathrm{nM}(\mathrm{ET}-1:+)$ to FTOWT as well as FTO ${ }^{\text {sikD }}$ cells from both PCTRL and WTD2 cell lines. Water served as mock-control (ET-1: -). (A) iPSC-CMs from the PCTRL cell line were stained for $\alpha$-actinin and DAPI. Images were taken using the FITC channel (green) as well as the DAPI channel (blue) at 400X magnification with an oil-immersion objective. (B) The cell sizes were measured with ImageJ. In pCTRL cells, significant increases in cell size were observed upon ET-1 treatment in FTOWT cells, whereas the cell size of FTOsikD cells is not significantly increased. The mean cell size of FTOsikD cells is significantly smaller after ET-1 stimulation compared to ET-1 treated FTOWT cells (C) Immunofluorescence images of ET-1 treated FTOWT and FTOsikD cells from the WTD2 cell line are presented with cells stained for $\alpha$-actinin and DAPI. Images were taken at 400X magnification. (D) In WTD2 cells, no significant hypertrophic growth is seen in FTOWT cells, but mean cell sizes identify a trend toward increased cell size in ET-1 stimulated FTOWT cells which seems less strong in FTOsikD cells. For cell size analysis of both cell lines, the mean of the cell sizes measured per slide was taken for statistical analysis. The mean with SEM is presented. t-test with Welch's correction was performed and significance levels are shown per condition in comparison to mock control. The number of all counted cells per condition on all slides together is given in the respective bars. 


\subsubsection{ET-1 stimulation does not affect FTO expression per se}

In order to determine whether ET-1 treatment alone is sufficient to affect FTO expression, iPSC-CMs of the WTD2 cell line were treated with either 3 nM ET-1 or water as mock control for 48 hours (Figure 3.31) and the FTO protein level analyzed with Western blot.

No difference in the FTO protein level can be seen between ET-1 stimulated or mock-treated cells $(p=0.8)$ (Figure $3.31 \mathrm{~B})$. Therefore ET-1 stimulation seems to not affect FTO per se and potential effects seen in later experiments are mediated by the FTO knockdown and not by ET-1 itself.

A

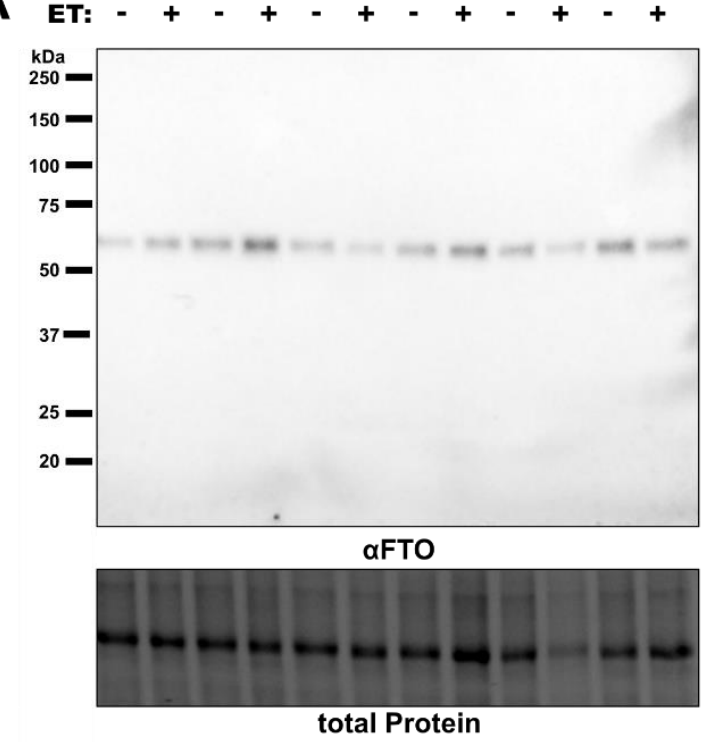

B

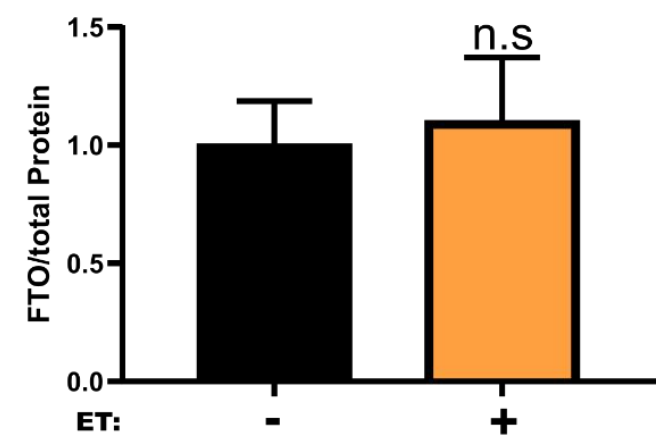

Figure 3.31 The FTO protein level is unchanged upon ET-1 treatment

iPSC-CMs of the WTD2 cell line were treated with ET-1 or water (mock, -) for 48 hours and the protein levels of FTO were examined with Western blot. (A) Western blot image of FTO protein bands with the respective total protein lanes. Each lane represents an independent biological replicate of each group (- = mock, $n=6 ;+=E T-1$ treated, $n=6$ ) (B) No significant effect on the FTO protein level can be seen upon ET-1 treatment. The quantified bands, normalized against total protein lanes, were analyzed and protein level changes after ET-1 treatment calculated. Water treated cells served as mock control. The mean with SEM is presented. Different wells from one differentiation experiment served as biological replicates ( $n=6$ per group) and t-test with Welch's correction was applied to determine the significance level.

\subsubsection{Molecular analysis of attenuated cell growth by ANP level assessment}

ANP is a typical cardiac protein marker being expressed and upregulated in hypertrophic CMs in response to cell stress (Kessler-Icekson et al. 2002). Since ANP expression increases with increased stress, it can serve as a marker indicating the severity of the hypertrophic growth. 
To further examine the attenuated cell growth seen in FTO ${ }^{\text {sikD }}$ cells of the $\mathrm{PCTRL}$ cell line compared to FTOWT cells after ET-1 stimulation, the lower hypertrophic response of treated cells was evaluated on a molecular level by measuring the atrial natriuretic peptide (ANP) protein expression in those groups (Figure 3.32). The protein level was assessed by Western blot, and the result normalized to the FTOWT - mock group (Figure $3.32 \mathrm{~B}$ ).

A strong increase in ANP levels occurs in cells treated with ET- 1 by almost $290 \%$ in the FTOWT group $(p<0.0001)$. The ANP level also rises in the FTO ${ }^{\text {sikD }}$ cells when ET-1 stimulated, but only by $140 \%(p=0.04)$. (Figure $3.32 \mathrm{~B}$ ). It comes clear that the ANP level is significantly lower in $\mathrm{FTO}^{\text {sikD }}$ cells when stimulated with ET-1 compared to the respective ET-1 stimulated FTOWT controls by almost $40 \%(p=0.05)$, potentially indicating less stress correlating to the attenuated cell growth.

It was further observed that the knockdown of FTO does not lead to the induction of ANP protein expression, as seen by a change of only $\sim 4 \%$ in mock treated FTO ${ }^{\text {sikD }}$ cells in comparison to mock treated FTO ${ }^{\mathrm{WT}}$ cells $(p>0.9)$ (Figure $3.32 \mathrm{~B}$ ). This may indicate that the transfection of siRNAs does not exert cellular stress triggering ANP expression and is a good model for protein expression manipulation in iPSC-CMs.

A

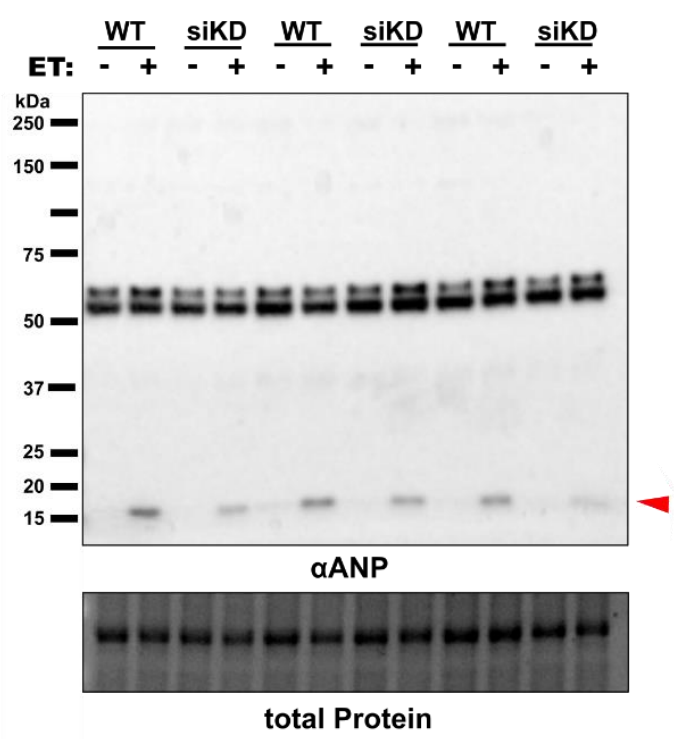

B

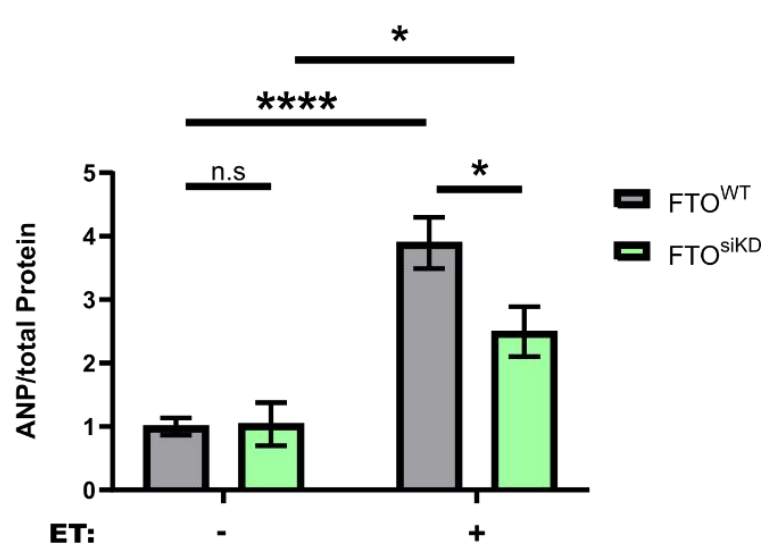

Figure 3.32 FTO-KD leads to less strong ANP expression

(A) Western blot was used to assess the protein level of ANP in iPSC-CMs of the pCTRL cell line in response to ET-1 treatment. The ANP-specific protein band at $17 \mathrm{kDa}$ is indicated with a red arrow. Unspecific bands with unknown origin occurred at 55 and $58 \mathrm{kDa}$. Corresponding total protein lanes used for normalization are presented. (B) $17 \mathrm{kDa}$ Protein bands of ANP were quantified, normalized against total protein lanes and ANP level changes calculated. In FTOWT cells, ANP expression is significantly elevated upon ET-1 induced hypertrophic stress response $(p=<0.0001)$. In FTOsikD cells 
ANP is also elevated, but to a lesser extent, which is in line with the attenuated hypertrophic growth observed with fluorescence microscopy. The mean with SEM is presented. An enhanced ANP blot with covered unspecific bands is shown in Appendix Figure 5.4.

In summary, the FTO knockdown related effect on hypertrophic responses of iPSC-CMs strongly suggest that FTO manipulation, and therefore m6A RNA methylation manipulation may be a potential suitable target for treatment of hypertrophy.

To determine whether the FTO downregulation has therapeutic potential, FTO manipulation was investigated in an in vivo mouse model, to elucidate the effect on a systems level. 


\subsection{The conditional Fto-knockout mouse}

Given the fact, that knockdown of FTO in iPSC-CMs of the PCTRL cell line resulted in attenuated hypertrophy, and also mean cell sizes of ET-1 treated FTO ${ }^{\text {sikD }}$ WTD2 cells were smaller than ET-1 stimulated FTO ${ }^{\mathrm{WT}}$ it was of interest, to elucidate if the attenuated hypertrophy would also be seen in vivo. Therefore, mice with a cardiomyocyte specific Fto knockout (Fto ${ }^{\text {ckO }}$ ) were generated. In a cre-lox system, exons flanked by loxP-sites are excised by crerecombinase (Agah et al. 1997; Nagy 2000). In this specific mouse model, the third exon of Fto is flanked by loxP-sites in FTO flox animals, and when crossed to Myh6 ${ }^{\text {Cre }}$ animals this exon is excised by cre-recombinase (Figure 3.33 A). Exon 3 of Fto encodes $\sim 40 \%$ of the protein and deletion of exon 3 results in a frame-shift of the downstream exons resulting in early termination of translation (Gao et al. 2010). As the cre-recombinase is under control of the Myosin Heavy Chain 6 (Myh6) promotor, expressed in CMs only (Agah et al. 1997), it is a CM specific conditional knockout (cKO). All mice were bred homozygous for the loxP-allele resulting in a homozygous cKO.

\subsubsection{Validation of the cardiomyocyte-specific knockout of Fto}

To verify a successful $\mathrm{KO}$ of Fto, first a successful recombination of loxP-sites via the crerecombinase and thus the exon 3 deletion was investigated with legacy PCR (Figure 3.33 B), then the Fto-mRNA levels were analyzed via RT-qPCR (Figure $3.33 \mathrm{C}$ ), and finally the depletion of FTO protein levels was verified with Western blot (Figure 3.33 D, E).

To determine if exon 3 was successfully excised by the cre-recombinase in Fto ${ }^{\text {cko }}$ animals, DNA was isolated from the left ventricles of mice and analyzed with legacy PCR. Littermate animals negative for cre-recombinase served as controls (Flox ${ }^{\text {Control }}$ ). A forward primer (FTO_genFOR), binding in front of the loxP-site was used in combination with a reverse primer, to bind downstream of exon 3 (FTO_recombination_R) (Figure 3.33 A). This would result in a PCR product of $>2 \mathrm{~kb}$ size for the not-excised Flox ${ }^{\text {Control }}$ exon, too long to be amplified. When recombination occurs, exon excision brings FTO_recombination_R binding site in proximity of the FTO_genFOR binding site, resulting in a PCR product of $345 \mathrm{bp}$ in FTO ${ }^{\mathrm{ckO}}$ mice. The results of the legacy PCR demonstrated that the recombination was successful, as only Fto ${ }^{\text {cKO }}$ animals show bands at $\sim 345 \mathrm{bp}$.

As mentioned above, the Fto-mRNA levels were investigated. RNA was isolated from CMs and the Fto-mRNA level assessed via RT-qPCR. A clear reduction (Figure 3.33 C) of Fto-mRNA

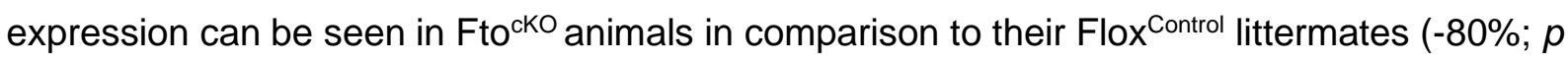
$<0.0001)$. The fact that residual Fto-mRNA is detected in Fto ${ }^{\mathrm{cKO}}$ samples may derive from the 
$\mathrm{CM}$ isolation procedure with some non-cardiomyocytes being isolated along the CMs. Furthermore, cre-mediated KO is only $80-90 \%$ effective, as shown by Agah et al. (Agah et al. 1997).

To determine the FTO expression in the $\mathrm{Fto}^{\mathrm{ckO}}$ mouse model, Western blotting was performed using protein from isolated $\mathrm{CMs}$ from either $\mathrm{Fto}^{\mathrm{cKO}}$ animals or their respective Flox ${ }^{\mathrm{Control}}$ littermates (Figure 3.33 D, E).

The FTO protein level is significantly $(p=0.02)$ decreased in Fto ${ }^{\text {cKO }}$ animals by $77 \%$ (Figure 3.33 E). Residual protein that could be detected in Fto ${ }^{\text {cKO }} \mathrm{CMs}$ may derive from remaining noncardiomyocytes, as previously explained. 
A
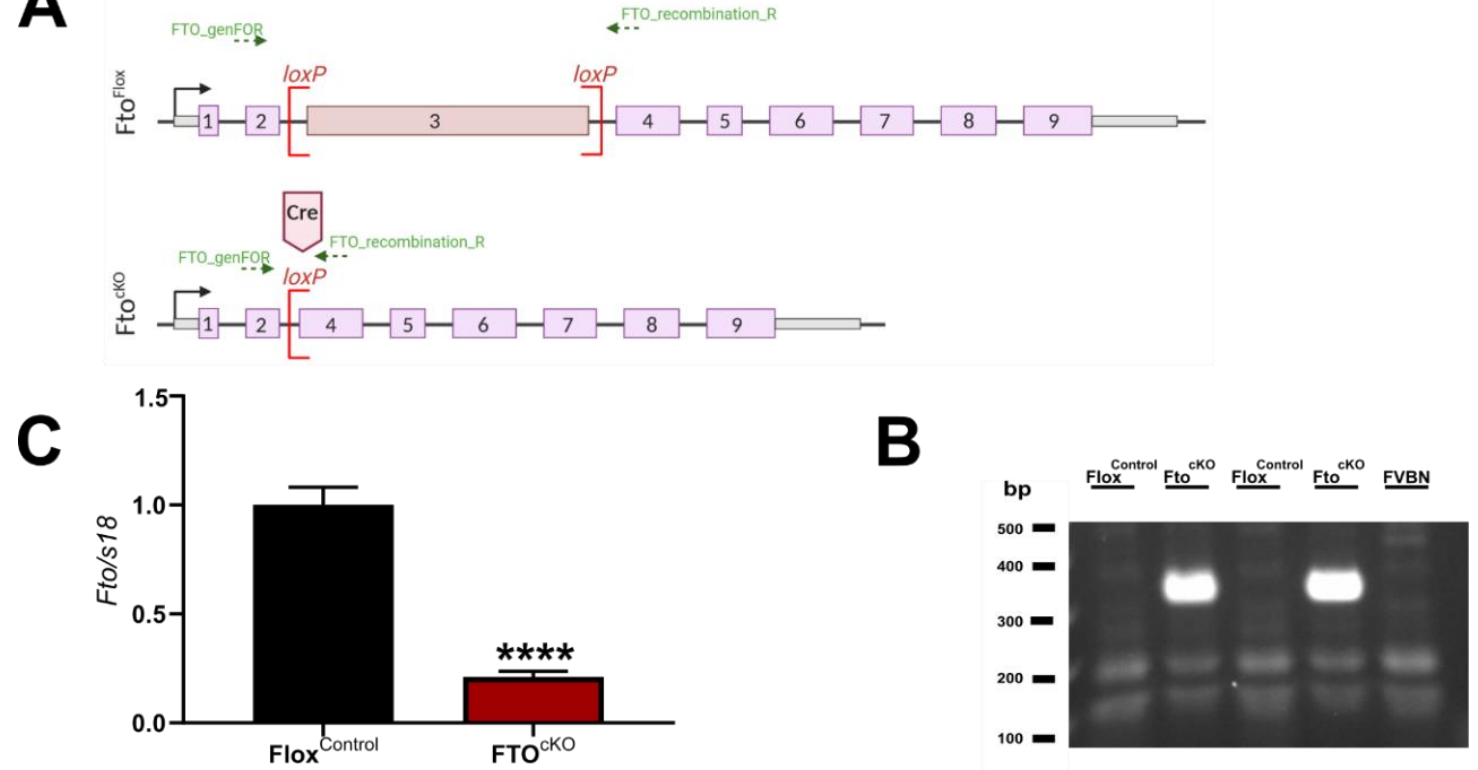

D

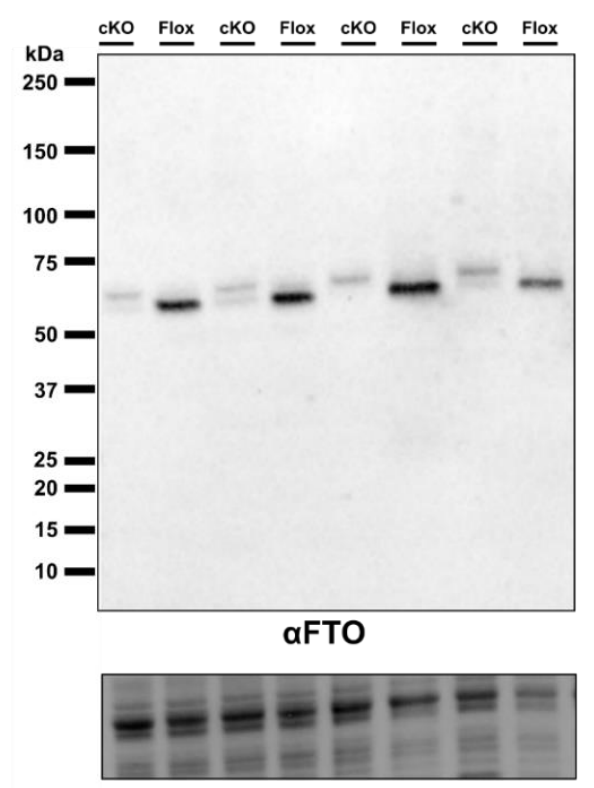

E

total Protein

Figure 3.33 Validation of the Fto ${ }^{\text {cKO }}$ mouse line

(A) Exon 3 of Fto is flanked by loxP-sites which are excised and re-joined by cre-recombinase resulting in a frame-shift of downstream exons. Exon sizes are to scale, introns and UTRs (grey) are not to scale for easier visualization. The primers for legacy PCR are indicated. (B) Excision of the floxed exon results in a $345 \mathrm{bp} \mathrm{PCR} \mathrm{product} \mathrm{which} \mathrm{otherwise} \mathrm{would} \mathrm{be} 2 \mathrm{~kb}$ in size. Homozygously floxed, cre-negative littermates (cre- $\mathrm{fl} / \mathrm{fl}$ ) are designated Flox ${ }^{\text {Control }}$ animals and do not produce the $345 \mathrm{bp}$ band in legacy PCR. Homozygously floxed cre-positive (cre+ fl/fl) FtockO animals produce the $345 \mathrm{bp}$ band, indicating successfully excision of exon 3. FVBN mice served as an independent WT control. (C) RT-qPCR was performed on RNA extracted from isolated CMs of FtockO mice as well as their Flox Control littermates. Cycles of Fto were normalized against s18, and the RNA expression level change calculated with the ddCt-method. t-test with Welch's correction was performed for statistical analysis. CMs from different mice served as biological replicates with $n=8$ for Flox Control and $n=6$ for Ftocko. Samples were pipetted in duplicates for technical replication. (D) Western blot images show a reduction of FTO in Fto ${ }^{\mathrm{ckO}}$ mice compared to Flox ${ }^{\mathrm{Control}}$ mice. The corresponding total protein lanes, used for normalization, are presented. (E) Specific FTO bands were normalized against total protein lanes and the relative protein level change of Ftocko mice compared to Flox Control calculated. The mean with SEM is presented. Protein extracted from different animals served as biological replicates $(n=4$ 
per group). t-test with Welch's correction was performed and significance level are shown above the bars.

\subsubsection{Phenotyping the knockout mouse model}

After the Fto ${ }^{\text {cKO }}$ mouse line was verified, it was necessary to determine whether the Fto-cKO influences the viability, heart dimensions, and heart function of the mice on a basal level, meaning otherwise healthy animals not challenged with TAC or other treatment. Therefore, the survival of Fto ${ }^{\mathrm{cKO}}$ mice was monitored and the cardiac parameters of these animals analyzed with echocardiography. The designated Flox ${ }^{\text {Control }}$ littermates served as control. Since crerecombinase was described to have cardiotoxic effects and to induce age-dependent DCM (Agah et al. 1997; Rehmani et al. 2019), for the following experiments, cre positive animals without flox-sites (cre+ +/+), from now on named Cre ${ }^{\text {Control }}$, served as additional controls to rule out potential cre-mediated effects.

\subsubsection{Survival analysis}

Fto $^{\text {ckO }}$ animals do not appear to have an initially higher mortality that can be connected to the knockout of Fto (Figure 3.34). The survival of $\mathrm{Fto}^{\mathrm{ckO}}$ animals bearing the knockout is comparable to the $\mathrm{Cre}{ }^{\mathrm{Control}}$ animals. The median survival of Fto ${ }^{\mathrm{CKO}}$ animals is 230.5 days, while the median survival of Cre ${ }^{\text {Control }}$ animals is 265 days $(p=0.7)$. Since Flox ${ }^{\mathrm{Control}+}$ animals, carrying no recombinase, all survived until the last day of monitoring (day 400), the observed mortality of Fto ${ }^{\mathrm{cKO}}$ and $\mathrm{Cre}{ }^{\mathrm{Control}}$ animals derives most likely from Cre-recombinase effects as already described by Agah et al. (Agah et al. 1997). 


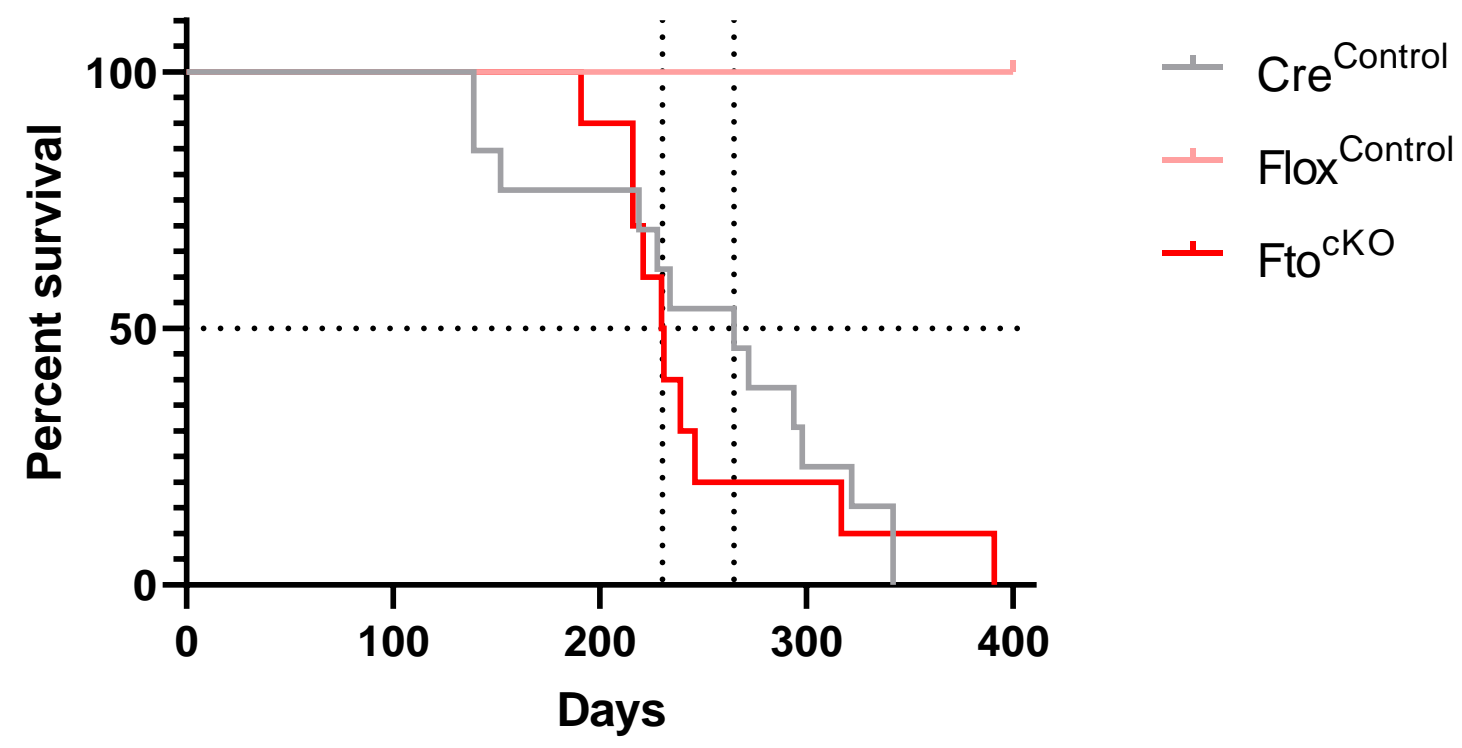

Figure 3.34 Basal survival is not affected by the Fto-KO until 8-months of age

A Kaplan-Meier curve depicts the percentage of animals surviving over a time period of 400 days of monitoring on a basal level. The KO of Fto does not appear to have a basal impact on survival and only a cre-derived effect is seen, indicated by similar survival rates in the Ftocko and Crecontrol group $(p=0.7)$. The median survival of Crecontrol animals is 265 days, while Ftocko animals survive for a median of 230.5 days. All animals belonging to the Flox Control group were alive at the final day of monitoring (day 400). $n=13$ for Cre Control, $n=10$ for Flox Control, and FtockO. The Mantel-Cox test was used to determine significances between the groups which were as follows: Cre ${ }^{\text {Control vs. Flox }}{ }^{\text {Control }} p$ $<0.0001$; Flox ${ }^{\text {Control vs. Fto }}{ }^{\mathrm{KKO}} p<0.0001 ;$ Cre $^{\text {Control }}$ vs. Fto ${ }^{\mathrm{cKO}} p=0.7$.

\subsubsection{Cardiac performance and morphometry of knockout-mice}

To further elucidate if depletion of Fto alters the cardiac phenotype on a basal level, Ftocko mice were echocardiographically analyzed every two months for half a year. Cre- negative littermates (Flox ${ }^{\text {Control }}$ ) as well as Cre-WT animals (Cre ${ }^{\text {Control }}$ ) served as controls (Figure 3.35).

Until 2 months of age the cardiac performance is comparable between all three groups (Figure 3.35 A). With the progression of time, the ejection fraction (EF) of the Fto ${ }^{\mathrm{ckO}}$ animals decreased significantly (Figure 3.35 D left panel) and the left ventricle showed dilatative growth, as demonstrated by an increased left ventricular interdimensional diameter (LVID) in comparison to the control groups (Figure 3.35 $\mathrm{D}$ right panel). This remodeling process was seen first after 4 months of age and continues over time (Figure 3.35 B, C, D). The ventricular wall thickness, signified by the anterior wall thickness (AwTh), increased in both control groups (Figure 3.35 D, middle panel), with the Cre ${ }^{\text {Control }}$ showing the greatest increase, potentially indicating the phenotypic change due to cre-recombinase activity and its cardiotoxicity (Agah et al. 1997; Rehmani et al. 2019). The AwTh in Ftocko animals began to increase over a time period of 4 
months but stayed constant afterwards until the time point of 6 months, at which the AwTh of $\mathrm{Cre}^{\text {Control }}$ at this time point was higher (Figure 3.35 $\mathrm{D}$ middle panel).

A general tendency towards concentric hypertrophic growth was observed in both control groups, which had no effect on the cardiac performance as the EF was mainly preserved. In Fto ${ }^{\text {ckO }}$ mice on the other hand, a dilatative phenotype developed over time, leading to a reduced EF and indicating an impaired cardiac performance.

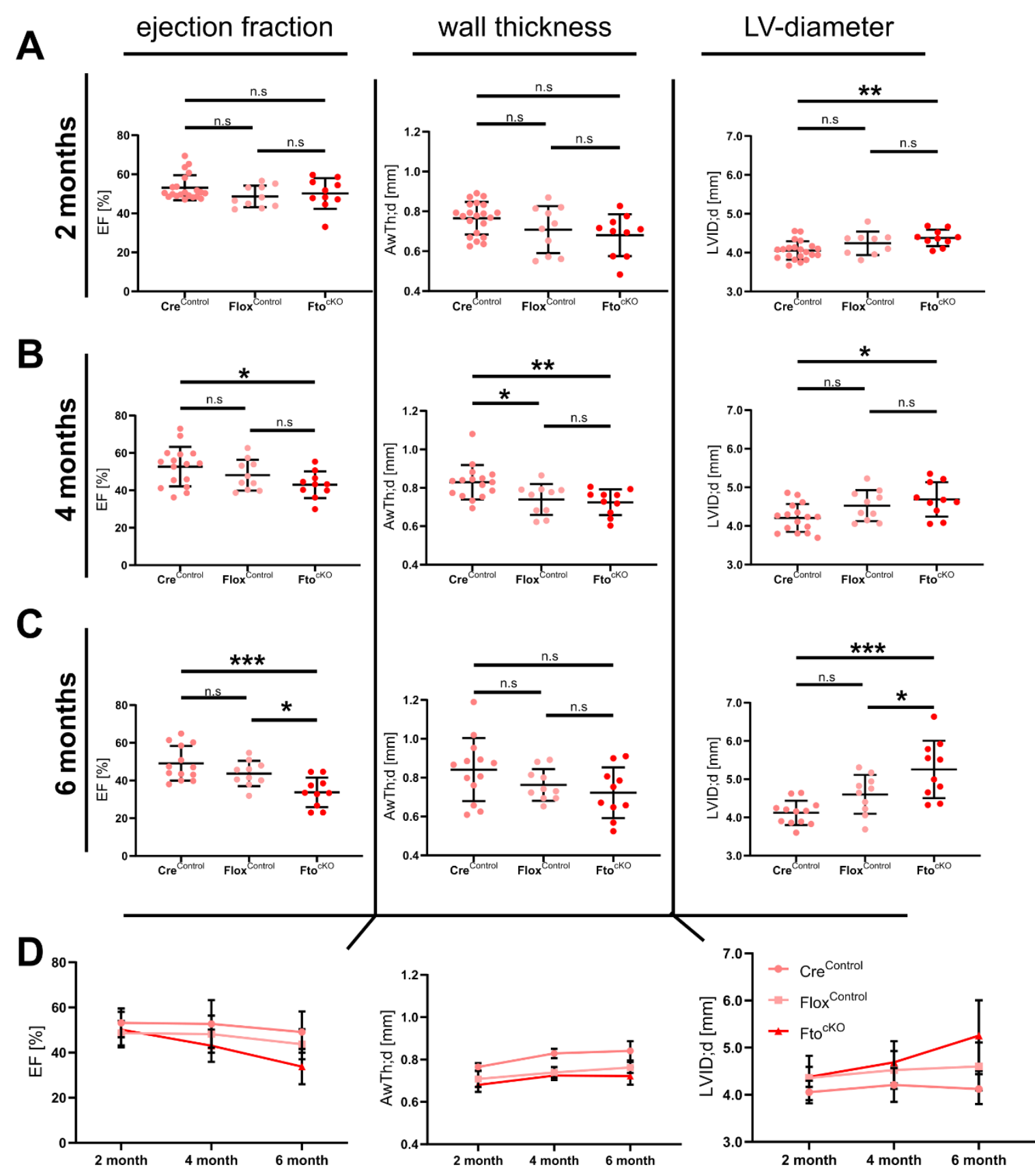

Figure 3.35 Basal echocardiographic analysis of Fto ${ }^{\text {cKO }}$ animals suggests a dilatative basal phenotype

The overall cardiac phenotypes of FtockO and control mice were assessed with echocardiography at an age of two months (A), four months (B) and six months (C). The ejection fraction (EF), anterior 
wall thickness (AwTh) and left ventricular interdimensional diameter (LVID) are shown. Each point represents the measured value from one animal. One-way ANOVA together with Tukey's multiple comparisons test were used. The error bars indicate SD, and significance levels are indicated. (D) The means \pm SD of the parameters are presented over time. A trend of decreasing EF in FtockO animals with increased dilatation (LVID) is seen whereas both control groups do not show significant remodeling.

\subsubsection{Response to TAC-induced hypertrophy in the Fto-cKO mouse model}

To further assess how the KO of Fto may affect cardiac remodeling in response to $\mathrm{PO}$, the TAC model was used to induce HT. Although a basal phenotype can be observed in FtockO animals over time (Figure 3.35), almost no difference between the Fto ${ }^{\mathrm{cKO}}$ and control (Flox ${ }^{\mathrm{Control}}$ Cre ${ }^{\text {Control) }}$ groups was observed at two months of age, the time point at which TAC surgery is performed. Assessment of the post-TAC cardiac changes occurs both one week and four weeks after TAC, at which the mice are approximately 3 months of age. As the Flox ${ }^{\text {Control }}$ group did not show a specific phenotype and were comparable to the Cre ${ }^{\text {Control }}$ group, and with respect to the reduction of animal numbers, further experiments were conducted with only the Cre ${ }^{\text {Control }}$ animals serving as the control group.

\subsubsection{TAC surgery with a 27-G needle}

To induce PO by aortic constriction, differently sized placeholder needles are used to tie the constriction knot. The unit for needle sizes are gauge numbers; the smaller the gauge number, the bigger the diameter of the needle and the lesser the constriction of the aorta. Due to a high consistency of both the results and the induction of severe hypertrophy, the standard needle size of 27 gauge (27G) was initially used to perform TAC surgery in Fto ${ }^{\text {cKO }}$ and Cre Control $^{\text {Con }}$ animals. Sham animals underwent the same surgery and procedure without constriction of the aorta and served as control group to rule out surgery-induced, inflammatory, or anesthesiaderived effects.

One week post-surgery (Figure 3.36), the EF was already substantially reduced in FtockO mice by $70 \%$ (corresponding to a difference of $36.9 \% ; p<0.0001$ ). In comparison, Cre ${ }^{\text {Control }}$ animals had a not significantly reduced EF by a difference of $13.7 \%(p=0.2)$ (Figure $3.36 \mathrm{~A})$. The wall thickness increased in both groups significantly to a similar extent $\left(0.32 \mathrm{~mm}\right.$ in Cre ${ }^{\text {Control }}, p=$ $0.02 ; 0.33 \mathrm{~mm}, p=0.0002$ in Fto ${ }^{\mathrm{cKO}}$ ) (Figure $3.36 \mathrm{~B}$ ). These values conform to the typical adaptation response of the heart to the increased pressure overload. The Cre ${ }^{\text {Control }}$ group shows almost no change in LVID $(-0.3 \mathrm{~mm}, p=0.8)$ whereas in Fto ${ }^{\mathrm{cKO}}$ animals a tendency toward increase in the LVID may be recognized $(0.51 \mathrm{~mm}, p=0.1)$ (Figure $3.36 \mathrm{C}$ ). The LVID of Fto ${ }^{\text {cKO }}$ animals is significantly bigger compared to Cre Control $^{\text {animals }}(p=0.009)$. Together 
with the increased wall thickness, this points to comparable hypertrophic remodeling of FtockO and Cre ${ }^{\text {Control }}$ animals, being of compensatory, concentric nature in Cre ${ }^{\text {Control animals with }}$ preserved $\mathrm{EF}$, whereas the Fto ${ }^{\mathrm{cKO}}$ animals show a worsened $\mathrm{EF}$ and dilatation and therefore undergoing faster progression to heart failure.

A ejection fraction

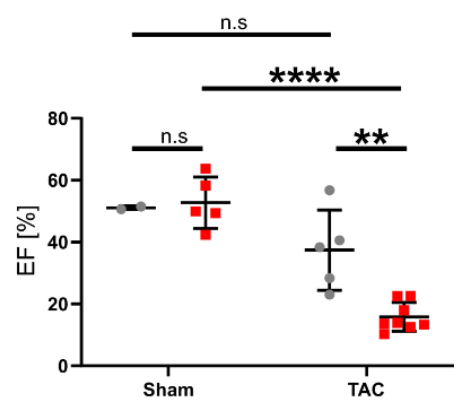

B

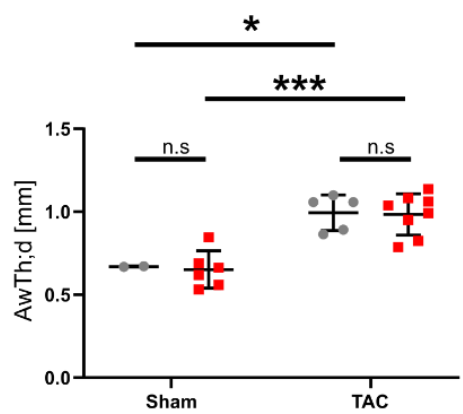

C LV interdimensional diameter
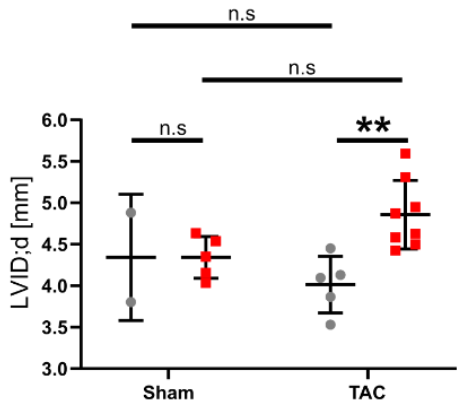

- $\mathrm{Cre}^{\text {Control }}$

- $\mathrm{Fto}^{\mathrm{ckO}}$

Figure 3.36 Echocardiographic characterization of Fto ${ }^{\mathrm{cKO}}$ animals one week after TAC with 27-G needle

Echocardiographic measurement was performed one week after TAC surgery. (A) The EF was impaired in Fto ${ }^{\mathrm{KKO}}$ animals but mainly preserved in $\mathrm{Cre}^{\mathrm{Control}}$ animals. (B) The AwTh was increased in both groups to a comparable extent. (C) The LVID was increased in response to TAC in FtockO animals in comparison to PO challenged Cre ${ }^{\text {Control }}$ animals. Each dot represents one animal, mean $\pm S D$ is given, two-way Anova with Tukey's correction for multiple comparison analysis was performed and significance levels between groups are indicated above measurements.

Four weeks post-TAC the observed echocardiographic parameters are even more dramatic (Figure 3.37). The EF is significantly decreased in both $\mathrm{Fto}^{\mathrm{cKO}}$ and $\mathrm{Cre}^{\mathrm{Control}}$ groups and showed strongest reduction in the Fto ${ }^{\text {cKO }}$ group by $85 \%(p<0.0001)$ (Figure $\left.3.37 \mathbf{A}\right)$. The AwTh is increased in Fto ${ }^{\mathrm{cKO}}$ animals $(0.19 \mathrm{~mm}, p=0.01)$ and a similar tendency may be recognized in Cre ${ }^{\text {Control }}$ animals $\left(0.15 \mathrm{~mm}, p=0.1\right.$ in Cre ${ }^{\text {Control }}$ ) (Figure $3.37 \mathrm{~B}$ ), which is less thick than seen after 1 week ( 0.3 mm) (Figure 3.36 B). This suggests maladaptive cardiac remodeling with a thinning of the wall occurring when PO stress persists. Big differences could be seen between the two groups when comparing LV diameter. After four weeks post-TAC, a mild increase in LVID of the Cre ${ }^{\text {Control }}$ group may be appreciated in response to TAC compared to the respective Sham group (LVID in end-diastole increased by $0.48 \mathrm{~mm}, p=0.3$ ) (Figure $3.37 \mathrm{C}$ ). This may indicate maladaptive remodeling occurring after the initial compensatory state one week after TAC with the concentric growth. The dilatation of the Fto ${ }^{\mathrm{cKO}}$ animals was significantly higher by $1.18 \mathrm{~mm}(p=0.0004)$ in comparison to the Fto ${ }^{\text {cKO }}$ Sham group (Figure $3.37 \mathrm{C}$ ), strongly suggesting a dilatative hypertrophic response. 

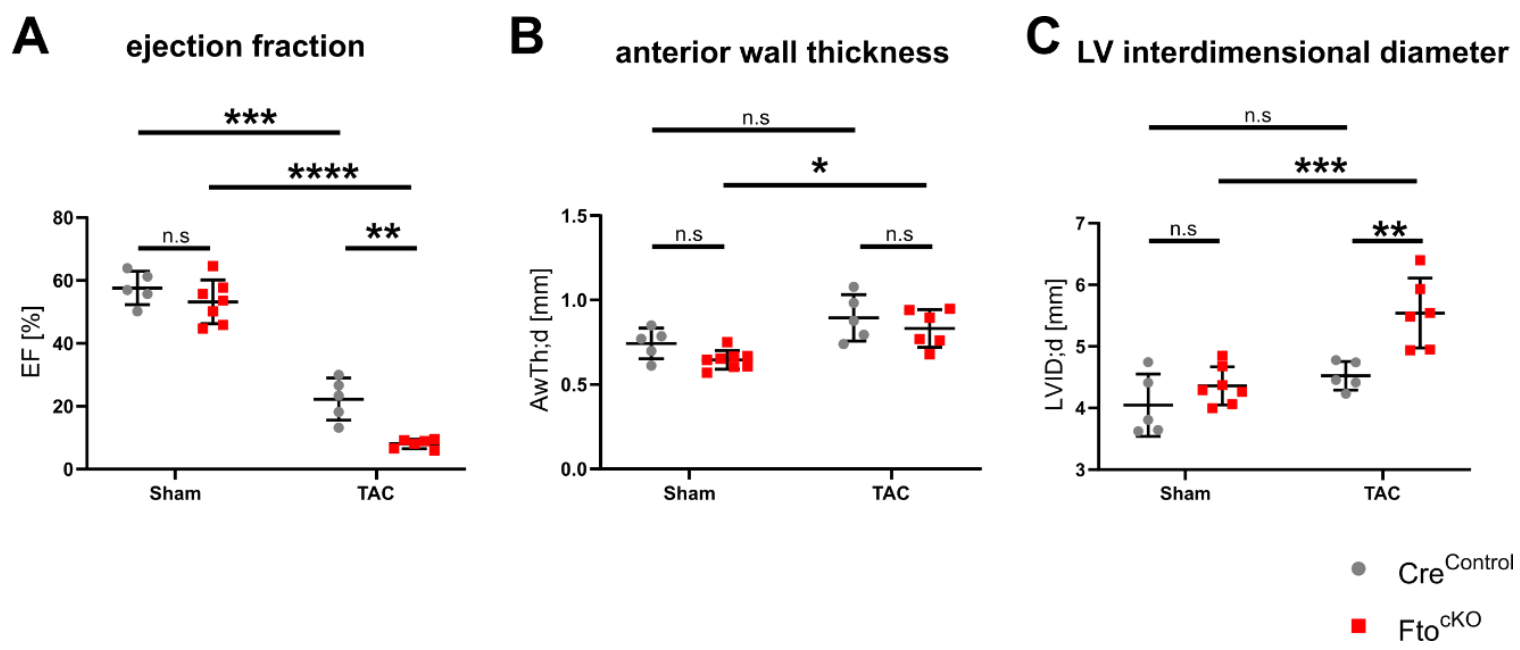

Figure 3.37 Echocardiographic characterization of Fto ${ }^{\mathrm{cKO}}$ animals 4 weeks after TAC surgery with a 27-G needle

FtockO animals and Cre Control animals were analyzed echocardiographically four weeks after TAC surgery. (A) The EF was impaired in Cre ${ }^{\text {Control }}$ animals but even more severely impaired in FtockO animals. (B) The AwTh was increased in both groups, but less thick than one-week post-TAC. (C) The LVID was significantly increased in the Fto ${ }^{\mathrm{cKO}}$ group. Each dot represents the measurement of one animal, mean \pm SD is given, two-way Anova with Tukey's correction for multiple comparison analysis was performed, and significance levels between groups indicated above measurements.

\subsubsection{TAC surgery with $26 \mathrm{G}$ needle}

A strong response to $\mathrm{PO}$ was seen for $\mathrm{Fto}^{\mathrm{cKO}}$ animals and $\mathrm{Cr} \mathrm{e}^{\mathrm{Control}}$ animals when performing TAC using a 27-G needle. In order to reduce the burden of the TAC intervention itself, additional experiments were performed using a 26-G needle. This was also in interest of planned gene expression analysis, which are then more responsive to the phenotype than to surgical effects. Finally, it was concentrated on the time point of one-week post-TAC, at which echocardiographic parameters were already changed in TAC animals, to ensure a larger pool of surviving animals

Echocardiographic analysis of the Fto ${ }^{\mathrm{cKO}}$ mice and Cre ${ }^{\text {Control }}$ mice one week after TAC with the 26-G needle (Figure 3.38) showed a result comparable to mice analyzed one week after TAC with the 27-G needle (Figure 3.36). In Cre ${ }^{\text {Control }}$ animals, the EF is preserved $(9.5 \%$ corresponding to a difference of $-4.8 \% ; p=0.6$ ) (Figure $3.38 \mathrm{~A}$ ), the AwTh is not significantly increased $(0.16 \mathrm{~mm} ; p=0.08)$ (Figure $3.38 \mathrm{~B}$ ), and the LV diameter is unchanged as well (by $0.13 \mathrm{~mm} ; p=0.9$ ) (Figure $3.38 \mathrm{C}$ ). The preserved EF may suggest compensatory, concentric

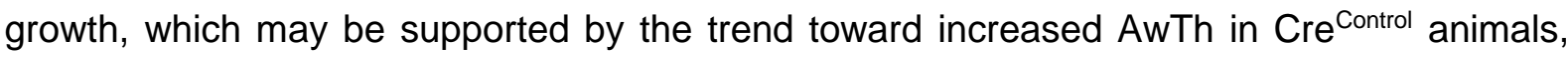
even if not significant. However, the opposite effects were observed in the Fto ${ }^{\mathrm{ckO}}$ group. The EF was significantly reduced by $48 \%$ (corresponding to a difference of $23.6 \%$; $p=0.0001$ ) (Figure $3.38 \mathrm{~A}$ ). The ventricle did not show a significant dilatation upon TAC compared to Sham Fto ${ }^{\mathrm{cKO}}$ animals $(0.41 \mathrm{~mm} ; p=0.2)$ (Figure $3.38 \mathrm{C}$ ), but when comparing TAC challenged groups, 
the LVID is significantly dilated $(p<0.0001)$ in $\mathrm{Fto}^{\mathrm{cKO}}$ animals compared to Cre ${ }^{\mathrm{Control}}$ animals. The AwTh of Fto ${ }^{\mathrm{cKO}}$ animals is unchanged in response to TAC $(-0.02 \mathrm{~mm} ; p>0.9)$ (Figure 3.38 B). The increased LVID and the unchanged wall thickness fit an early DCM-like heart failure phenotype, in which heart mass is increased, but wall thickness is normal (Figure 3.38).

A ejection fraction

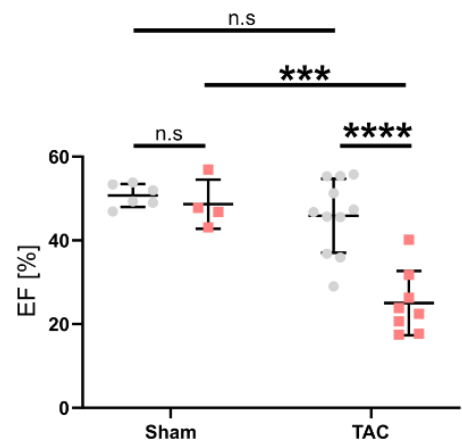

B

anterior wall thickness
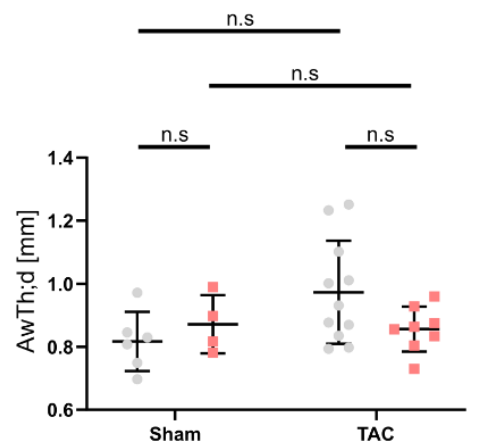

C LV interdimensional diameter

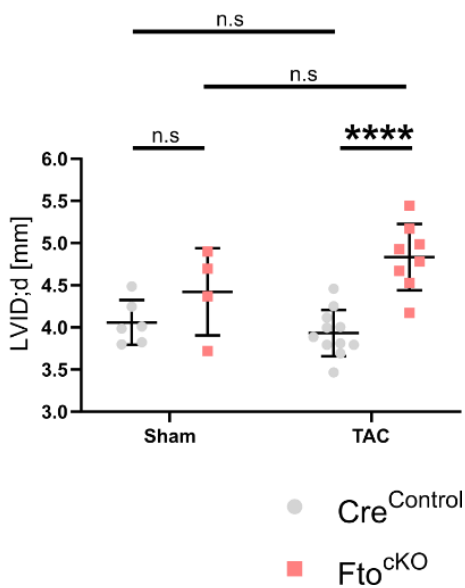

Figure 3.38 Echocardiographic characterization of Fto ${ }^{\mathrm{cKO}}$ animals one week after TAC with a 26-G needle

FtockO animals and Cre ${ }^{\text {Control }}$ animals were analyzed echocardiographically one week after TAC surgery using a 26-G needle. (A) The Cre ${ }^{\text {Control }}$ group showed parameters of compensated hypertrophy with a preserved EF, whereas the EF is significantly decreased in FtockO animals. (B) The AwTh is unchanged in both FtockO and Cre ${ }^{\text {Control }}$ animals upon TAC (C) The LVID is unchanged in response to TAC in Cre ${ }^{\text {Control }}$ animals, whereas it trended toward increase in FtockO animals. Although not significant when compared between TAC and Sham FtockO animals, the LV is significantly dilated in FtocKO animals compared to TAC challenged Cre ${ }^{\mathrm{Control}}$ animals. Each dot represents the measurement of one animal, mean \pm SD is given, two-way Anova with Tukey's correction for multiple comparison analysis was performed and significance levels between groups indicated above measurements.

\subsubsection{Fto FKO $^{\mathrm{cKO}}$ mice show different cardiac remodeling than $\mathrm{Cre}{ }^{\mathrm{Control}}$ mice}

To better understand the difference between the hypertrophic responses of $\mathrm{Fto}^{\mathrm{cKO}}$ and $\mathrm{Cre}$ Control animals in response to TAC induced $P O$, the ratio of $L V$ weight and tibia length (LV/TL) as well as the relative wall thickness (RWT) was calculated (Figure 3.39). An increase in the LV/TL ratio after TAC in comparison to baseline (Sham) is usually an indicator of hypertrophic growth. An increase in RWT in response to TAC indicates a concentric growth, a decrease an eccentric growth.

The relative wall thickness (RWT) is calculated by dividing the sum of the anterior wall thickness (AwTh) and posterior wall thickness (PwTh) by the LV inner dimension (LVID):

$$
\frac{A w T h+P w T h}{L V I D}=R W T
$$


The LV/TL ratio, calculated from Cre $\mathrm{Control}$ and Fto ${ }^{\mathrm{cKO}}$ animals (Figure $3.39 \mathrm{~A}$ ), increased in TAC animals compared to Sham animals in both groups. Even if not being significant, a tendency towards increase can be recognized suggesting hypertrophic growth.

The RWT increases in Cre ${ }^{\text {Control }}$ animals challenged with PO (Figure 3.39 B) significantly by 0.1 ( $p=0.008$ ), indicating concentric hypertrophy. In the Fto ${ }^{\text {ckO }}$ group, no change is seen of the RWT ( $p=0.5$ ), suggesting less concentric growth, and rather early dilatation of the LVs of these animals (Figure 3.38).

\section{A left ventricle/tibia length}

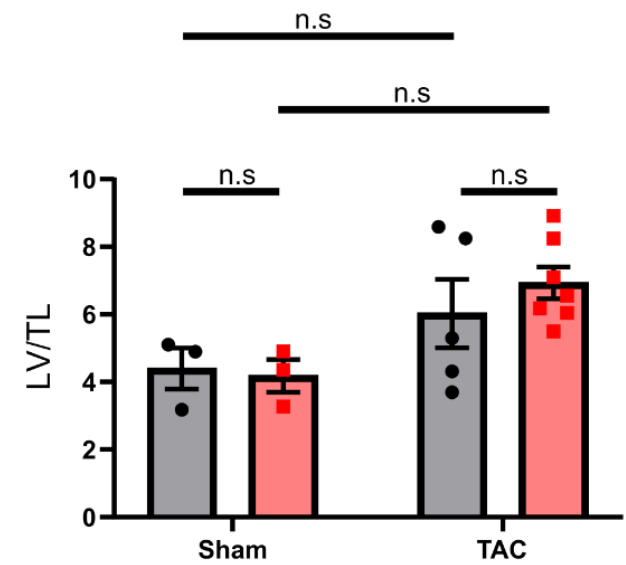

B

\section{relative wall thickness}

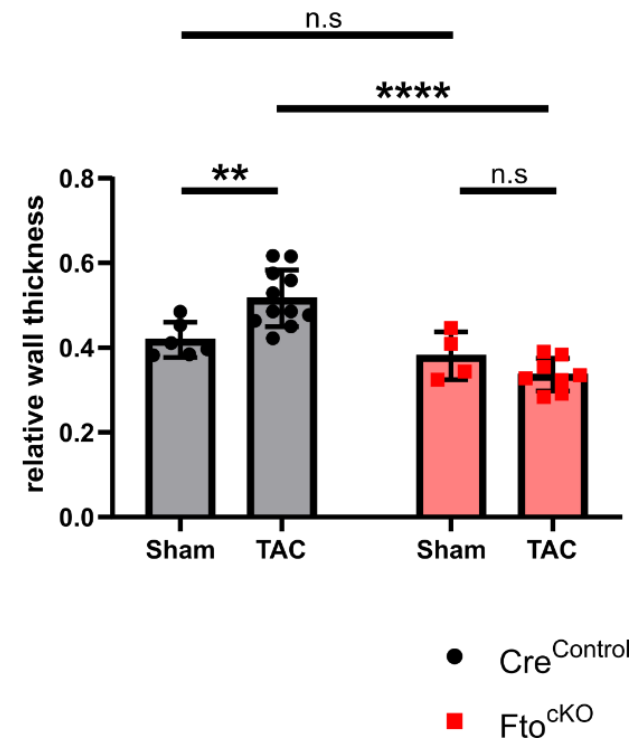

Figure 3.39 Fto-KO affects the hypertrophic response

(A) The LV/TL ratio shows tendencies of hypertrophy in both, FtockO as well as Crecontrol animals. Even if not significant, a trend can be recognized. (B) The relative wall thickness (RWT) demonstrates concentric hypertrophy in Cre ${ }^{\text {Control }}$ animals, whereas Fto ${ }^{\text {cKO }}$ animals show no changes in RWT. Each dot represents the measurement of one animal and bars were added to visualize the trends and changes better. Two-way Anova with Tukey's multiple comparison test was performed, and significance levels presented.

Ultimately it appears, that the cKO of Fto leads to a worsened adaptation to pressure overload with a strong decrease in EF, and a tendency towards early dilatative growth. Therefore, cardiac performance is impaired in animals bearing the Fto $\mathrm{KO}$ (Fto ${ }^{\mathrm{cKO}}$ ) in comparison to Cre ${ }^{\text {Control }}$ animals, in which compensated hypertrophy with preserved EF may be recognized. This maladaptive response in Ftocko mice in response to TAC is in line with the basal phenotype observed in these animals, but with a faster progression. 


\section{Discussion}

RNA methylation is an emerging field of research interest. It is studied extensively for the last decade after newly developed methods, such as MeRIP-seq (Dominissini et al. 2012), allowed the detection and characterization of $\mathrm{m} 6 \mathrm{~A}$ methylation and gave rise to the new term of 'epitranscriptomics'. m6A RNA methylation, as the most abundant epigenetic modification in RNA (Fu et al. 2014), is dynamic and reversible (Fu et al. 2014; Roundtree et al. 2017; Yang et al. 2018), and found in many classes of RNA, such as mRNA, tRNA, rRNA, miRNA or circular RNAs (Dominissini et al. 2012). As an additional layer of gene expression regulation, the epitranscriptome was investigated in various fields and diseases such as cancer ( $\mathrm{Li} Z$ et al. 2017; Zhang et al. 2017; Lan et al. 2019; Yue et al. 2019; Liu et al. 2020), neurodegenerative disorders (Hess et al. 2013; Widagdo and Anggono 2018; Han et al. 2020) or developmental biology (Boissel et al. 2009). Nevertheless, only little is known about its role in cardiovascular diseases (CVDs) and only a few studies reported on the role of m6A methylation in the heart, which mainly concentrated on in vitro studies and direct METTL3 manipulation (Dorn et al. 2019; Kmietczyk et al. 2019; Mathiyalagan et al. 2019). None of them provided a complete epitranscriptomic landscape of the m6A RNA methylation in mouse and human LV tissue which was the first goal of my study.

\subsection{The RNA methylation machinery allows dynamic methylation in cardiac tissues}

Previous studies already showed, that m6A RNA methylation can regulate gene expression post-transcriptionally in various cell types (Fu et al. 2014; Wang and He 2014). Therefore, I first wanted to analyze the m6A landscape in case of cardiac hypertrophy and heart failure, which was never reported by the time the project was started.

First, I could show that the RNA methylation machinery, consisting out of the methyltransferases METTL3 and METTL14 and the demethylase FTO, is present in mouse and human left ventricular (LV) tissue (Figure 3.1). This potentially allows the addition of $\mathrm{m} 6 \mathrm{~A}$ marks to adenosine of RNAs by METTL3 and METTL14 functioning as writer-proteins. The demethylase FTO, which acts as an eraser of the m6A mark, is present in LV of both species as well. Interestingly, the protein level of the writers and erasers was found to be mainly unchanged in diseased cardiac tissues, as shown by Western blot analysis of healthy and failing heart LV tissue of mouse and human (Figure 3.1, Appendix Figure 5.1). A similar observation was made by Kmietczyk et al., who reported METTL3 and FTO protein level being unchanged in human DCM hearts compared to healthy hearts (Kmietczyk et al. 2019). This finding contradicts the finding of Mathiyalagan et al., where a decrease in FTO levels was 
reported in failing ischemic hearts (Mathiyalagan et al. 2019). However, it must be mentioned that this decrease was seen in the infarction and peri-infarction zone only, whereas no such change occurred in non-ischemic tissue. As their study focused on ischemic cardiac myopathies (ICM) and the LVs used in my study are mainly of DCM nature or taken from noninfarction zones from ICM tissues, they represent more end-stage HF condition. This may explain these discrepancies and shows the putative versatility of the methylation machinery.

\subsection{The epitranscriptome of mouse and human hearts elucidated}

By applying MeRIP-Seq, the global m6A landscape (epitranscriptome) could be elucidated. Analysis of healthy mouse and human LV tissue showed that a significant number of all detected transcripts carries m6A marks. $24.09 \%$ of all transcripts were methylated in mouse LV tissue, and $14.6 \%$ in human LV tissue. Furthermore, the highly conserved consensus motif DRACH ( $D=A, G$ or $U, H=A, C$ or $U$ ) was identified to be the main methylation site in transcripts in cardiac tissues. This motif occurs approximately once every $\sim 57 \mathrm{nt}$ in mRNA, potentially allowing methylation of every transcript (Zaccara et al. 2019), and was already reported to be the main site for m6A methylation in RNA (Wei and Moss 1977; Linder et al. 2015). Additionally, the relative quantification of detected m6A peaks across all transcripts showed enrichment toward the transcription end site and the 3' UTR, a distribution reported similarly in other tissues (Dominissini et al. 2012; Meyer et al. 2012; Chang et al. 2017; Slobodin et al. 2017). This findings are in line with recent reports on relative enrichment of methylation marks in the UTRs in cell culture of neonatal rat cardiomyocytes (NRCM) (Dorn et al. 2019).

Interestingly, in mice a majority of the methylated transcripts carried the m6A marks in the CDS ( $52 \%)$ or 3' UTR and CDS $(25,6 \%)$ at the same time, and only a small amount in the 5 ' UTR and CDS ( 8\%) or exclusively in the 5' UTR ( 0.4\%) or 3' UTR ( 7,6\%) (Figure 3.3). In human tissue, also transcripts were mainly methylated in the CDS $(\sim 53,2 \%)$, and fewer in the 5 ' UTR and CDS at the same time $(\sim 3,5 \%)$, whereas a substantial amount was exclusively methylated within the 3' UTR (31,3\%) (Figure 3.3). Similar enrichment of m6A methylation marks was also presented in previous studies (Dominissini et al. 2012; Meyer et al. 2012; Ke et al. 2015). Ke et al. for example found more than $70 \%$ of all detected m6A marks in their study to be located in the last exon, which includes the 3' UTR, in various tissues (Ke et al. 2015).

Because of the distribution of methylation marks across the regulatory sites of transcripts, it was interesting to have a closer look at the functions of the genes whose transcripts were methylated either in the 5' UTR and CDS or exclusively in the 3' UTR (Figure 3.4). GO term analysis demonstrated that transcripts methylated in the $5^{\prime}$ UTR and CDS mainly participate in 
metabolic and catabolic processes, whereas 3' UTR methylated transcripts are involved in signal transduction pathways. This was found to be true for mouse and human transcripts. Furthermore, 550 transcripts are commonly methylated in both tissues (Figure 3.5). GO analysis showed that these transcripts are of cardiac specificity, as represented by GO clusters of 'heart development', 'circulatory system development' and 'vasculogenesis' as well as clusters representing metabolic processes, similar GO-terms associated with 5' UTR/CDS and 3' UTR methylated transcripts. Additionally, the GO term 'energy derivation by oxidation of organic compounds' may represent participation of methylated transcripts in mitochondrial function. Mitochondria are especially important in CMs due to the high demand of energy in these cells and mitochondrial dysfunction is associated with various CVDs, such as atherosclerosis, hypertrophy and heart failure (Siasos et al. 2018).

\subsection{Hypertrophy and heart failure were successfully induced in mice}

As demonstrated for the healthy heart, m6A RNA methylation is present in cardiac tissue and may have regulatory functions. Therefore, the role of $\mathrm{m} 6 \mathrm{~A}$ methylation in the development of cardiac HT and HF was investigated. To study HT and HF, the well described TAC surgery was performed in wildtype mice (deAlmeida et al. 2010). Echocardiographic analysis of LVs revealed successful induction of HT one week after the TAC surgery (Figure 3.6). Mice that received TAC surgery showed increased anterior wall thickness (AwTh) with a preserved EF, indicating the compensatory state where the hypertrophic growth sustains sufficient blood output of the pressure overloaded heart (Norton et al. 2002). 8 weeks after TAC surgery (Figure 3.7), the EF was significantly decreased in TAC animals despite a thickened wall compared to Sham animals, depicting the inability of the heart to preserve sufficient blood flow resulting in HF. Therefore, this model was suitable for the analysis of the transcriptome, as well as the epitranscriptome in the progression to heart failure.

\subsection{Transcriptomic changes in response to cardiac stress account for cardiac plasticity and remodeling processes}

NGS was performed to study the RNA expression in LVs of mice. Compared to Sham, in LV tissue of TAC-mice 217 genes were found to be differentially expressed upon hypertrophy, and 174 in heart failure $(\log 2 \mathrm{Fc} \geq 1$ or $\leq-1, P a d j \leq 0.05)$. Consistent with recent literature (Nomura et al. 2018), these transcripts mainly participate in pathways regulating cardiac plasticity and remodeling at both time points. Further comparison of these transcripts underlines the finding, that RNA expression of genes mainly influences the cardiac plasticity and structural response to cardiac stress. 71 genes were differentially deregulated similarly in cardiac HT and the HF 
state compared to Sham mice and GO term analysis of these revealed that they code for genes important in hypertrophic responses, remodeling, and cardiac plasticity (Figure 3.8).

The human disease was analyzed to a similar extent. Human LV tissue from heart explants of patients with HF, being of both ischemic and dilatative nature (ICM and DCM, respectively) was used for transcriptomic analysis. Transcripts deregulated in comparison to healthy human cardiac LV tissue were detected and further analyzed. Changes of the human end-stage HF transcriptome is consistent with the findings from mice, as the identified 228 differentially expressed genes from human HF tissue are mainly involved in pathways regulating heart morphology and remodeling (Figure 3.9). A hypertrophic state in the human model was not analyzed. Even though tissue of hypertrophic human hearts was available, those biopsies were rather small and not suitable for extraction of the big amount of RNA needed for MeRIP-Seq. Therefore, I concentrated on human end-stage HF tissue, where a sufficient amount of tissue was available due to complete ex-plantation of hearts which is performed in end-stage HF when no other options of treatment are available.

\subsection{The epitranscriptome changes in response to cardiac stress}

Transcriptomic changes of morphological and functional signatures have previously been correlated with stress-induced structural changes of the heart during the progression to heart failure (Nomura et al. 2018). To uncover the role of the epitranscriptome in HF development, m6A methylation changes in LV tissue of hypertrophic and failing hearts in comparison to healthy hearts was investigated. Analysis was performed similar to the MeRIP Sequencing applied to describe the basal landscape of m6A methylation in healthy heart tissues. It is important to mention, that the same cutoffs were applied for differential expression and differential methylation ( $\log 2 \mathrm{FC} \geq 1$ or $\leq-1$, Padj $\leq 0.05$ ) to make changes comparable and only investigate transcripts changed stably and strongly on expression and methylation level.

\subsubsection{Methylation changes outnumber expression changes by far}

Interestingly, the analysis of m6A RNA methylation in response to cardiac stress revealed that the change of the RNA methylation level exceeds the change of expression level by far. Many more transcripts were differentially methylated than differentially expressed in mouse HT (1611 vs 217 respectively, Figure 3.10 A), mouse HF (1182 vs 174 respectively, Figure 3.12 A) as well as in human HF (1249 vs 228 respectively, Figure 3.14 A). Interestingly, changes in methylation occurred despite the fact that the methylation machinery was mainly unchanged at the protein level. Only the METTL3 protein level was decreased in HF tissue of mice, potentially explaining why more hypomethylated peaks were detected in murine HF tissue. However, since also in 
human HF tissue more hypomethylated peaks were detected, but the METTL3 protein level was unchanged, observed hypomethylation may not solely be mediated by METTL3. The mechanisms by which 'writers' and 'eraser' discriminate which RNAs to methylate or to demethylate remains largely unknown (Zaccara et al. 2019). Yet, there is first evidence that the proteins of the methylation machinery can be modified post-translationally by themselves (Yeo and O'Rahilly 2012; Wang $P$ et al. 2016; Du et al. 2018). For example, METTL3 was shown to have reduced methyltransferase activity upon SUMOylation, decreasing $\mathrm{m} 6 \mathrm{~A}$ levels of mRNAs (Du et al. 2018). This is one possible explanation for the observed considerable differential methylation changes even though the protein levels of the methylation machinery itself remained mainly unchanged throughout disease progression. Yet, more research is needed to elucidate how the m6A epitranscriptome is installed in a selective manner in CMs.

Nevertheless, the significant methylation changes observed upon progression to heart failure mainly occur in the CDS and 3' UTR in mouse HT as well as mouse and human HF (Figure 3.10 A, Figure 3.12 A, Figure 3.14 A). This is in line with the earlier described epitranscriptomic landscape in healthy heart tissues, as well as with the assumption of Ke et al. that the enrichment in 3' UTR allows potential regulation of the 3' UTR (Ke et al. 2015). The 3' UTR bears consensus motifs for microRNA (miRNA) interaction (Jonas and Izaurralde 2015), therefore, m6A methylation at or near this miRNA binding motifs may affect miRNA mediated mRNA regulation (Fazi and Fatica 2019). In addition, miRNAs can be m6A methylated themselves (Berulava et al. 2015b), which adds yet another layer to the epigenetic control of cellular processes, and would be interesting to investigate more specifically in the future.

It was previously described (Feng et al. 2015), that apoptotic, dying CMs excrete cardiac RNAs. This extracellular RNA (exRNA) was shown to induce cytokines and to trigger an inflammatory response in a paracrine manner in other heart cells via Toll like receptor 7 . The later subsequently leads to MAPK and NFkB pathway induction and hence inflammation. This mechanism was developed by cells to react to pathological RNA, for example viral RNA, and to evade viral replication (Brencicova and Diebold 2013). Although it is unknown how cells distinguish between foreign RNA and intrinsic RNA (Feng et al. 2015), it is hypothesized that m6A marks abrogate an immune stimulatory effect of exRNAs (Koski et al. 2004; Karikó et al. 2008). Therefore, a potential effect of the many methylated transcripts seen in my study may be to prevent inflammation, even though this may only be true for the hypermethylated transcripts, as hypomethylated transcripts would not be masked for their inflammatory potential. 


\subsubsection{Only a small number of transcripts is affected at the methylation and expression level at the same time}

Even though many more transcripts were differentially methylated than expressed, still a notable amount was also differentially expressed (217 mouse HT, 174 mouse HF, 228 human $\mathrm{HF}$ ). Only very few transcripts were deregulated at the expression and methylation level at the same time when the same cutoff was applied, more specifically only 78 in mouse HT, 47 in mouse HF and 30 in human HF (Figure 3.10 B, Figure 3.12 B, Figure 3.14 B respectively). GO term analysis revealed that these transcripts code mainly for proteins involved in remodeling processes and cardiac plasticity, similar to pathways found to be affected by RNA expression. It is important to mention, that in case of transcripts that changed expression levels and methylation degree, one cannot rule out that methylation impacts the level of the same transcript. RNA methylation was already proposed to have an impact on RNA decay rate and stability by protection from or tagging for degradation (Zaccara et al. 2019; Lee et al. 2020). This process may either be mediated by reader proteins, recruiting nucleases, or by direct conformational changes of methylated RNA, the so called 'm6A switch' (Liu et al. 2015). The methylation dependent mechanisms may result in their protection from, or recognition for decay (Wang et al. 2014; Wang et al. 2015). Moreover, splicing may affect transcript abundance, as wrongly spliced products are recognized for degradation (Hilleren and Parker 2003; Fasken and Corbett 2009). Therefore, RNA stability influencing processes may take place for the transcripts that changed their methylation and expression level at the same time. In summary, RNA methylation may alter the expression level of transcripts by regulating their half-life time, thus indirectly affecting cardiac remodeling. Even more, the fetal gene program is re-expressed in progression to heart failure (Frey and Olson 2003), and m6A methylation may affect this process. Translation regulation or altered alternative splicing (Wang et al. 2015; Slobodin et al. 2017) may be the mechanism how m6A affects fetal re-expression. In line with above mentioned hypothesis, fetal splice variants were shown to be present in cardiac hypertrophy (Ames et al. 2013).

\subsubsection{Transcripts changed at their methylation level reveal substantially different Functions than those changed at expression level - a fast response mechanism}

Pathway analysis of the transcripts that were only changed at their methylation level demonstrated that they participate in substantially different cellular functions compared to differentially expressed mRNAs. Where expression changes mainly affect cardiac plasticity and structural changes, differentially methylated transcripts participate mainly in catabolic and 
metabolic processes as well as signal transduction (Figure 3.11, Figure 3.13, Figure 3.15). More specifically, transcripts being hypomethylated were found to be coding for metabolic and catabolic processes in mouse HT as well as in mouse and human HF, whereas in the late HF state mitochondrial pathways were found additionally. Hypermethylated transcripts were associated with GO terms of signal transduction in mouse HT and HF as well as human HF. In the late disease stage (mouse and human HF), a few remodeling pathways were found in addition to signal transduction pathways for hypermethylated transcripts.

These findings suggest a fast response mechanism, with which cells can rapidly adapt to cellular stress. Expression changes need time to establish (de Nadal et al. 2011), whereas m6A methylation is a more rapid process and methylation changes have been reported to be induced already shortly after stress induction (Engel et al. 2018). This would explain why the dynamic and reversible RNA methylation mainly affects catabolic and metabolic processes, as well as signal transduction pathways. By epitranscriptomic changes, these networks could be fine-tuned and regulated in a rather short time, hence allowing fast response of cells to their need upon stress induction, like energy conservation (catabolic/metabolic) and rapid signaling. The importance of kinase-regulated signaling and MAPK pathways in hypertrophy has been well described (Liu et al. 2012; van Berlo et al. 2013). Since these pathways were affected by RNA methylation, this may indicate the importance of RNA methylation in cardiac diseases. Two recent studies support the involvement of RNA methylation in cardiac stress response. Dorn et al., found hypertrophy-dependent methylation of mRNAs encoding kinases (Dorn et al. 2019), and Kmietczyk et al. described enrichment of genes that regulate signal transduction to be methylated (Kmietczyk et al. 2019).

Even more specifically, methylated transcripts detected in my study encode several transcription factors (FOXO1, FOXO4, Elf5A), epigenetic proteins (SMYD1, DICER1, RBM20) and other regulators of gene expression (i.e ERK, MDM2). Therefore, m6A methylation may influence the early steps of gene expression, which are also important for the fast adaptation of cells to external stimuli.

In late stage HF, methylation changed transcripts were also associated with pathways that belong to remodeling processes. The reason for that may be that many cellular processes in CMs in the failing heart situation are deregulated and impaired. Because of that, structural pathways may be regulated by methylation without the need of expression change as the cell tries to compensate for the stress. 


\subsection{4 m6A RNA methylation affects translation}

Previous studies in cardiology have shown, that protein expression does not always correlate with their corresponding mRNA abundance (Brundel et al. 2001; Su et al. 2015), especially in response to pathological stimuli. Given that a majority of transcripts was altered only at their methylation level without expression being affected, other mechanisms than m6A influencing mRNA expression and hence protein abundance are likely to be present. In line with this, only mild correlations were observed between the methylation level and transcript abundance in healthy hearts (Figure 3.4 B, D), and also transcripts found differentially methylated and expressed in disease response do not correlate when analyzed in healthy tissue (Figure 3.17).

m6A RNA methylation is was reported to impact translation, for example by 5'cap independent translation initiation (Meyer et al. 2015), by reader protein mediated mechanisms (Shi $\mathrm{H}$ et al. 2017) or direct conformational changes of the RNA tertiary structure by the m6A switch (Liu et al. 2015). One of the most likely mechanisms may be that m6A methylation impacts translation by affecting ribosome occupancy (Bodi et al. 2015; Meyer et al. 2015), analyzed in this study via polysomal profiling.

225 transcripts were found to be differentially translated in the polysomal fraction in mouse heart failure in comparison to healthy control tissue (Figure 3.18). Interestingly, transcripts depleted from the polysome fraction were mainly found associated with GO terms of metabolic and catabolic pathways, similar to hypomethylated transcripts in the disease state (Figure 3.13). Furthermore, mRNAs enriched in the polysome fraction were coding for proteins involved in GO terms of structural pathways and more importantly signal transduction, similar to those found to be hypermethylated (Figure 3.13). A correlation analysis of methylation changes and polysome occupancy was performed and confirmed that the methylation level of mRNAs significantly correlates with their translation in murine HF tissue $(r=0.37, p=2.2 \mathrm{e}-16)$ (Figure 3.19). Due to sample limitation, only failing hearts from mice were analyzed in polysome profiling. To obtain information on human data, orthologous genes in humans that were correlated between their m6A and translation, were analyzed (Figure 3.20). Just as in mice, the cross-species analysis demonstrated a significant positive correlation between RNA methylation and translation rate. Even more, those transcripts reveal cardiac specificity suggesting a potential role of methylation on translation in cardiac tissues.

\subsubsection{A potential mechanism of transcription independent translation control}

Since methylation levels correlated with the translation, a mechanism of transcription independent translation control mediated by m6A RNA methylation was hypothesized. Thereby, a methylation increase, or decrease may lead to changed protein levels of the 
according genes. To test the hypothesis, the data was screened for cardio-specific transcripts that were differentially methylated and differentially translated by the polysome, but otherwise unchanged at their abundance (expression level). Investigation of the protein level of one interesting target was possible. Calmodulin1 (CALM1) changed protein level in response to HF dependent on the mRNA methylation level only in both mouse and human. Calm1 is a calcium ion sensor especially important in cardiac contraction coupling (Boczek et al. 2016; Yu et al. 2016). Calm1 was unchanged at the expression level in response to heart failure but was hypomethylated in the MeRIP data. Hypomethylation was confirmed by independent RTqPCR. The protein level of this target was significantly decreased in both, mouse and human tissue (Figure 3.21, Figure 3.22) when analyzed with Western blot, leading to the conclusion that hypomethylation may negatively influence translation. These findings suggest that, independent of mRNA abundance, the translation is directly affected by the methylation state. This may explain the discrepancy between protein level and mRNA level described in other studies (Brundel et al. 2001; Su et al. 2015).

Such effect may be mediated by RNA binding proteins (RBP), so called 'reader' proteins in the cytoplasm. The typical m6A reader protein YTHDF1 was shown to bind to the translation factor elf3, which recruits the small ribosomal subunit to enhance translation (Wang et al. 2015). Another possible mechanism is the previously discovered 5'cap independent translation (Meyer et al. 2015). Upon methylation of the 5' UTR, elf3 was shown to directly bind to the translation start site without the usual requirement of elf4E binding that normally recruits elf3 to the 5' UTR (Jackson et al. 2010).

4.5.6 m6A methylation in hypertrophy and heart failure may act in a 'fastresponse' mechanism by accelerating translation and turnover

Interestingly, hypomethylated and polysome depleted genes are involved in metabolic and catabolic processes, whereas, genes that were hypermethylated and enriched in the polysome are involved in signal transduction. It was previously described, that demethylation increases RNA stability whereas methylation reduces the half-life time of transcripts (Wang et al. 2014), tagging them for degradation. This suggests a passive mechanism for polysomal enrichment of hypomethylated and polysomal depletion of hypermethylated transcripts. However, in this study only a negligible number of transcripts was differentially methylated and expressed at the same time (Figure 3.10, Figure 3.12, Figure 3.14). Therefore, the later mechanism could explain only a small portion of the observations here.

Previous studies found stably expressed genes, such as "housekeeping genes" or ribosomal proteins, to be de-enriched in m6A (hypomethylated) (Schwartz et al. 2014). So, in my case, 
the hypomethylated transcripts may be more stable and have a longer half-life time, but do not accumulate as they are not constantly newly transcribed. This would make sense given that mainly pathways for energy conservation mechanisms (catabolic/metabolic) were affected. These energy conservation mechanisms are critical in diseased CMs and mRNAs coding for these mechanisms are constantly needed. In other words, hypomethylation leads to increased mRNA stability, enabling constant translation without the need of de novo transcription. In contrast, hypermethylated transcripts mainly code for signal transduction pathways and, therefore, may participate in the fast response mechanism. With hypermethylation, the transcript turnover may be accelerated by a m6A-dependent shorter half-life time (Du et al. 2016; Ke et al. 2017), but at the same time this methylation marks may tag the mRNAs for increased translation, as seen by enrichment in polysomes (Figure 3.18).

Taken together, hypomethylation might increase stability and transcripts affected are those continuously needed. Thus, they do not need to be newly synthesized but are stored in the cytoplasm or at cell compartments, allowing continuous rather than fast translation (Lewis et al. 2018). Lewis et al. already hypothesized such regulation mechanism in CMs. They could show that in CMs ribosomes are also present at the sarcomeres, and that mRNAs coding for sarcomeric proteins are collocated at those ribosomes allowing better maintenance of sarcomeric renewal. However, how exactly those mRNAs are selectively transported and stored remains unknown. m6A methylation of sarcomeric transcripts may be an explanation for organization of sarcomeric maintenance. Due to the big size of $\mathrm{CMs}$, methylation dependent intracellular distribution of mRNAs would be a suitable mechanism to keep stable transcripts with a long half-life time in the cell's cytosol. Thereby, newly synthesized mRNA does not need to be transported long ways from the nucleus to the far ends of myocytes (Lewis et al. 2018). As the RNA methylation machinery is mainly present in the nucleus (Bokar et al. 1997; Zheng et al. 2013; Meyer and Jaffrey 2017), and RNA methylation mainly occurs co-transcriptionally (Barbieri et al. 2017; Bertero et al. 2018; Huang et al. 2019), this mechanism seems even more plausible to be adapted in CMs. Due to their size with long distance from the nucleus to outer parts of the cytosol, the mRNAs fate may be decided already in the nucleus. On the other hand, RNA can also be methylated in the cytoplasm. It was shown in neurons that methylation changes happened in the synapses far away from the nucleus (Merkurjev et al. 2018). Hence, this mechanism may additionally take place in CMs where different methylation degrees of mRNAs directly at the sarcomeres may help in maintaining sarcomeric integrity.

In contrast to hypomethylation, described hypermethylated and ribosome-dense (polysomal) transcripts are involved in signal transduction pathways like 'regulation of calcium-mediated signaling', 'response to ischemia' or apoptotic signaling pathways (Figure 3.12, Figure 3.18). The observed reduced half-life time and polysome formation suggests a stress-dependent fast- 
response mechanism with a faster turnover rate on the one hand, and a 'burst-like' translation, on the other hand. Hence, having a shorter half-life time, these transcripts have a faster turnover rate (Ke et al. 2017) helping the cells to adapt quickly to signal transduction regulation, which again reflects the fast response mechanism mentioned before. Additional induction of translation by m6A marks may ensure that those transcripts are processed by ribosomes before being degraded. The enrichment of genes participating in signaling pathways in the polysome fraction, could possibly represent the higher readthrough of those transcripts. A possible mechanism of higher translation rate of hypermethylated transcripts may be the recently discovered impact of m6A RNA on liquid-liquid phase separation (LLPS) (Ries et al. 2019). Ries et.al. showed that the m6A RNA reader proteins YTHDF1-3 contain low complexity domains and can interact with each other when incubated with RNA. When incubated with m6A methylated RNA, this effect was enhanced promoting phase separation. These membraneless compartments may be used by cells to store or degrade RNA, or to transport RNAs to specific locations within the cell over longer distance (Langdon and Gladfelter 2018), protecting them from degradation during transport time. In addition to LLPS, YTHDF1-3 may also participate in the hypothesized mechanisms of fast response with fast turnover by their innate functions recently described (Du et al. 2016; Shi $\mathrm{H}$ et al. 2017). It was shown, that YTHDF3 together with DF1 increases mRNA translation (Shi $\mathrm{H}$ et al. 2017) while DF2 leads to mRNA decay by accelerating de-adenylation (Du et al. 2016). Shi et al. proposed a similar mechanism, where methylated RNA, exported from the nucleus, is first recognized by YTHDF3 or a DF3-DF1 complex. Additional binding of YTHDF2 and therefore partitioning of the mRNA between these three readers would then lead to decay by de-adenylation after DF3 mediated translation. This would support my hypothesis that this mechanism of fast-translation with fastturnover (Figure 4.1) is also in place in cardiac tissues, explaining how the hypermethylated transcripts in this study are participating in fast response.

The mechanism of cytosolic reader mediated translation control especially in CMs is further underlined by an interesting study, where they identified 1148 RNA-binding proteins (RBPs), of which 393 are uniquely found in CMs (Liao et al. 2016). These RBPs, acting as reader proteins, were described to participate in regulation of metabolic and catabolic processes and in cardio-specific diseases. Furthermore, many contained low complexity domains, therefore allowing granule formation. Another study revealed, that RBPs can interact with target sequences at 5' UTRs and 3' UTRs, as well as intronic and exonic regions (de Bruin et al. 2017) and were especially described to regulate splicing in cardiac remodeling response (Xu et al. 2005; Giudice et al. 2014). Although it is unknown how exactly m6A may regulate RBP binding, either by direct conformational changes (m6A switch) or other mechanisms, its involvement in the process underlines the importance of $\mathrm{m} 6 \mathrm{~A}$ in $\mathrm{CMs}$ and hence cardiac diseases. 
Another hypothetic mechanism of enhanced translation is mRNA looping. It was shown that METTL3, normally acting as a writer of the m6A code, can also act as a reader (Choe et al. 2018). METTL3 can bind to the methylated sites at the 3' UTR and stop codon and interact with the translation factor elf3, which binds in the 5' UTR, leading to mRNA looping. mRNA circularization allows easy ribosomal re-entry and thus fast repetitive translation.

It is important to mention that conclusions on translation from polysome profiling are limited due to its technical nature as only ribosome-dense transcripts are covered whereas mRNAs translated by single or only a few ribosomes are missed from the analysis. It is likely that hypomethylated transcripts encoding catabolic and metabolic proteins are translated continuously and slower by normal ribosomal read-through, whereas hypermethylated transcripts coding for signal transduction pathways are enriched in the polysome, being highly translated before their degradation (Figure 4.1). 


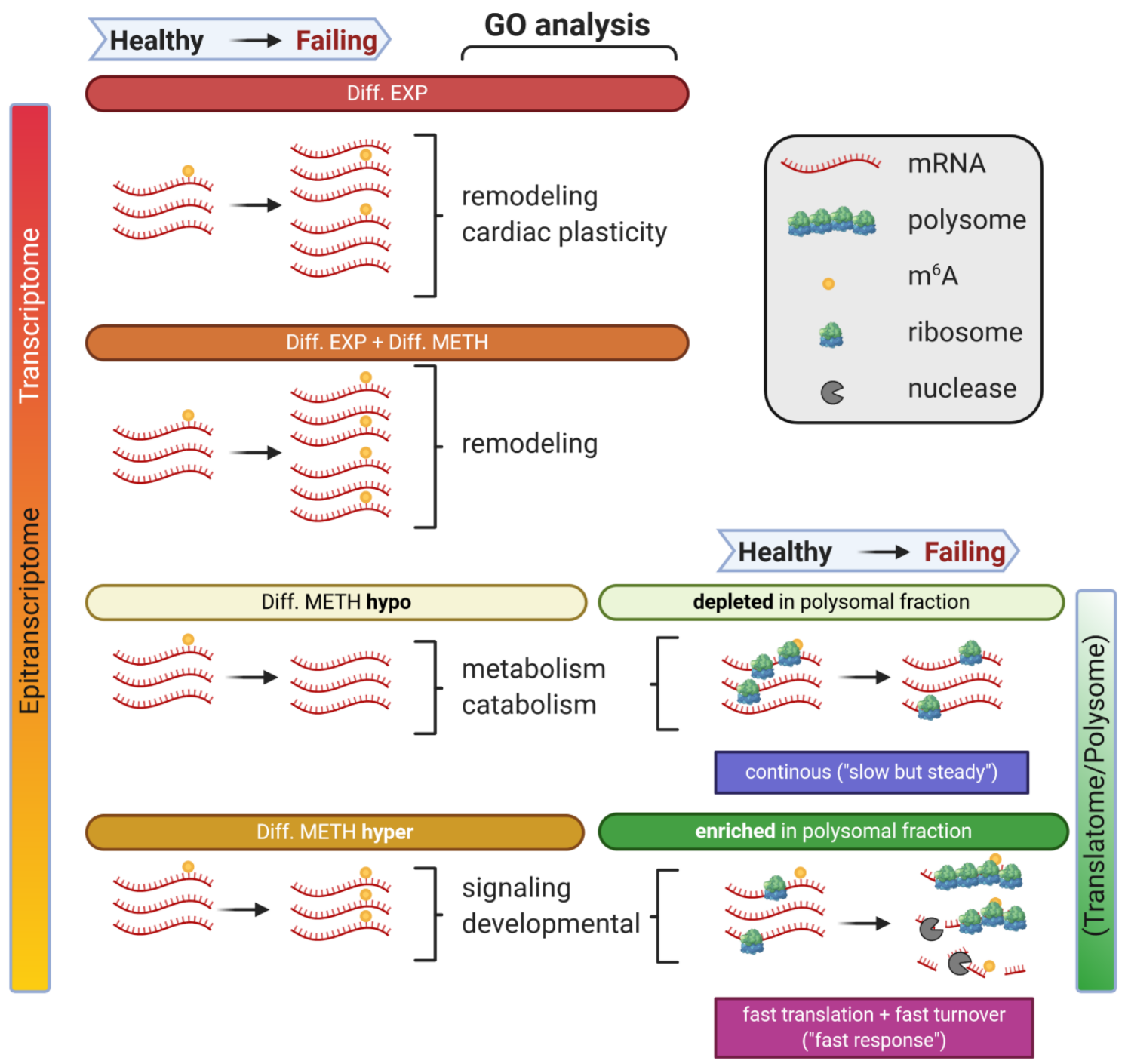

Figure 4.1 Transcripts changed at the methylation level are substantially different from those changed at the expression level - a potential fast-translation with fast-turnover mechanism Transcripts differentially expressed in heart failure were mainly found in GO terms of remodeling and cardiac plasticity mechanisms, those differentially methylated were found in GO terms of metabolism and catabolism when hypomethylated and in signal transduction and developmental processes when hypermethylated. Transcripts differentially changed at the expression and methylation level at the same time were found associated with GO terms similar to GO terms found for differentially expressed transcripts. Interestingly, transcripts only changed at the methylation level correlated with polysomal occupancy, hence translation rate. Hypomethylated transcripts code for similar GO terms as those depleted in the polysomal fraction. The hypomethylation may increase their half-life time and slow their turnover, so that they do not need to be newly transcribed allowing a continuous, 'slow but steady' translation. This especially makes sense for the found metabolic and catabolic processes, which allows the cell to conserve energy. In line with this, transcripts hypermethylated were found associated with similar GO terms like those being enriched in the polysome. The hypermethylation may induce translation, hence polysomal enrichment, and a fast turnover at the same time. This would ensure a 'fast response' mechanisms, supported by the fact that they were coding for signal transduction and developmental processes. With this, cells can quickly adapt to signaling changes and cope with stress stimuli in a fast manner. 


\subsection{FTO affects the hypertrophic response in vitro and in vivo}

Due to the notable RNA-methylation changes observed in mouse and human heart tissue during the progression to $\mathrm{HF}$, it was of further interest to investigate how manipulation of the m6A methylation machinery may affect disease progression. The m6A demethylase FTO has high expression levels in cardiac ventricles (Boissel et al. 2009) and was also detected in mouse and human cardiac LV tissue in my study (Figure 3.1). For investigation of a potential therapeutic approach of $\mathrm{m} 6 \mathrm{~A}$ methylation manipulation on cardiac disease progression, the m6A demethylase FTO was silenced in vitro using a siRNA-mediated approach and depleted in vivo using a Fto knockout mouse model.

\subsubsection{Silencing of FTO attenuates hypertrophy in vitro}

Functional analysis of FTO was first performed using human iPS-Cells (iPSCs). iPSCs are a widely used tool for in vitro studies nowadays (Malik and Rao 2013). Due to their ability to differentiate into different germ layers (Jaenisch and Young 2008), they could successfully be differentiated to beating iPSC-CMs with a well-established protocol (Lian et al. 2013). Mature CMs were obtained 60 days after differentiation initiation. For this time point, a stable cardiospecific gene expression was described previously (Shinozawa et al. 2012).

To investigate cardiac hypertrophy in vitro, iPSC-CMs were treated with Endothelin-1 (ET-1). ET-1 binds extracellular receptors inducing HT by downstream transcription factor activation and stimulates among others the renin-angiotensin-aldosterone system (Moreau et al. 1997; Gray 1998; Adiarto et al. 2012; Bupha-Intr et al. 2012; Zlabinger et al. 2019) as well as activates the CaMkll pathway (Moreau et al. 1997). A concentration of $3 \mathrm{nM}$ of ET-1 was determined sufficient to induce significant cell growth (Figure 3.23) leading to significant increase in ANP level by almost $290 \%$ (Figure 3.32). ANP is a peptide expressed and upregulated in hypertrophic CMs in response to cell stress (Kessler-Icekson et al. 2002). The successful induction of HT via ET-1 in my cells demonstrates the suitability of this model for investigations on remodeling in vitro.

Next, a successful siRNA-mediated knockdown of FTO was established in this study (Figure 3.25). siRNAs act as silencing compounds via the RNAi mechanism. siRNAs assemble with the RNA-induced silencing complex (RISC), which leads to binding of complementary mRNAs. The binding results in nucleolytic degradation of the mRNA by the RNase $\mathrm{H}$ Argonaute (Zhang 2013). In contrast to a knockout (KO), RNAi leads to a transient knockdown (KD) or silencing of a gene. Since the generation of a KO in iPSCs by for example CRISPR/Cas is rather time consuming, and may reduce the ability of IPSCs to differentiate into e.g. CMs (Chaterji et al. 
2017; Keller et al. 2018), the siRNA mediated knockdown approach was decided to use in my study since it allows silencing of genes in already differentiated iPSC-CMs.

I demonstrated on the RNA and protein level that transfection of the iPSC-CMs with FTOspecific siRNAs for $48 \mathrm{~h}$ was sufficient to silence FTO (Figure 3.25). This should potentially lead to global hypermethylation, as the demethylase activity of FTO is diminished. Unfortunately, due to sample limitations and a high demand of RNA for immunocytochemistry methods I could not prove a change of m6A level upon siRNA mediated silencing of FTO in the cells. Interestingly, cell growth is attenuated in FTO ${ }^{\text {sikD }}$ cells in comparison to scramble treated FTOWT cells upon ET-1 stimulation. It is important to mention, that I also verified that FTO is silenced during the whole time of hypertrophic induction (Figure 3.27, Figure 3.29) and that ET1 treatment does not influence the FTO level per-se (Section 3.2.5.2). Therefore, observed effects should be attributed to the robust silencing of FTO. Furthermore, ANP expression is decreased in $\mathrm{FTO}^{\text {sikD }}$ cells compared to FTOWT cells (Figure 3.32). This leads to the conclusion, that FTO depletion attenuates hypertrophy in vitro.

\subsubsection{A cardiomyocyte-specific Fto knockout in vivo results in a mild cardiac phenotype}

The previously described conditional Fto-KO mouse (Nagy 2000) was used to investigate the role of FTO in CMs in vivo. The successful $\mathrm{KO}$ of Fto was validated on genome, transcriptome and protein level. Cre mediated excision of exon 3 of Fto, flanked by loxP-sites, and the further recombination of loxP-sites was demonstrated to be successful by legacy PCR (Figure 3.33). In Fto ${ }^{\mathrm{ckO}}$ mice, Fto mRNA level was significantly reduced in comparison to Flox ${ }^{\text {Control mice }}$ ( $\mathrm{c}$ $0.0001)$ which finally led to significantly decreased FTO protein level by $77 \%(p=0.02)$ in these mice (Figure 3.33).

It was expected that the Fto ${ }^{\mathrm{KKO}}$ should lead to a global increase in m6A level. Surprisingly, the analysis of m6A level by an immunocytochemical assay did not show changed m6A RNA levels in Fto ${ }^{\mathrm{ckO}}$ mice (Appendix Figure 5.5). This might be due to methodological problems such as cell type heterogeneity. The heart is composed of many cells, with cardiomyocytes (CMs), fibroblasts (FBs) and endothelial cells (ECs) being the most abundant (Zhou and Pu 2016). Even though CMs account for $\sim 70-85 \%$ of the total mammalian heart volume (Anversa et al. 1980; Tang et al. 2009; Zhou and Pu 2016), they only constitute 30-40\% of the total amount of cells (Nag 1980; Banerjee et al. 2007; Walsh et al. 2010; Pinto et al. 2016). This is due to their relatively large size compared to for example FBs or ECs. In this study, a CM specific KO approach was used, so ideally RNA from isolated CMs should be used for further analysis. However, the CM isolation process involves several digestion steps at room temperature, 
which might lead to RNA degradation to which hypomethylated and hypermethylated RNAs are differently susceptible. Furthermore, recent research suggested that m6A may not be the main substrate of FTO (Zaccara et al. 2019), as Fto-KO did not lead to increased m6A levels in mouse brain (Hess et al. 2013) or embryos (Mauer et al. 2017). Recent findings suggest that m6Am might be the main substrate for FTO (Mauer et al. 2017). Even though a more specific effect of FTO on m6Am was shown, an effect of FTO on m6A cannot be excluded. For example, a $\sim 20 \%$ increase in $\mathrm{m6A}$ levels was reported upon FTO depletion in leukemia (Li Z et al. 2017). Although, FTO was shown to not erase m6A on a sequence specific manner (Wei and Moss 1977; Jia et al. 2011; Zou et al. 2016), it still has specificity since methylation changes were observed only for m6Am and m6A (Jia et al. 2011). Furthermore, m6A methylation is mediated by the complex interplay of the writers and erasers, and it cannot be ruled out that other players of the methylation machinery may affect $\mathrm{m} 6 \mathrm{~A}$ levels in response to FTO depletion. For example, the writers may be downregulated or methylate RNA to a lesser extent to adapt for potential m6A level changes by FTO manipulation.

Even though no global m6A change was observed, analysis of the heart morphology by echocardiographical analysis over half a year shows a mild cardiac phenotype in Fto ${ }^{\text {ckO }}$ mice. Cardiac performance is unchanged and comparable between $\mathrm{Fto}^{\mathrm{cKO}}$, Flox ${ }^{\mathrm{Control}}$ and $\mathrm{Cre}^{\mathrm{Control}}$ groups until 2 months of age as seen by similar ejection fractions (EF), wall thickness (AwTh) and ventricular dimension (LVID). After 4 months of age, a mild hypertrophic growth is present in Cre ${ }^{\text {Control }}$ mice as seen by a mild increase of the AwTh. This is most likely a Cre-mediated effect, as already described by Rehmani et.al. (Rehmani et al. 2019). Rehmani and colleagues have shown that cre-recombinase activity may induce cardiomyopathies with aging (Rehmani et al. 2019). After 6 months, the onset of dilatation is also seen in Fto ${ }^{\mathrm{ckO}}$ mice, indicated by increased LVID. The EF is slightly impaired and reduced in these animals compared to Flox ${ }^{\text {Control }}$ and Cre ${ }^{\text {Control }}$ mice. As Rehmani et al. described a DCM Phenotype induced by creexpression in mice, appropriate cre-control animals need to be used in studies, which was done in my case. Therefore, the even more severe dilatative change observed in Ftocko mice is more likely to be KO-dependent, since the cardiac phenotype in Cre ${ }^{\text {Control }}$ animals was less strong. Even though an effect is seen, this does not interfere with the experiments, as the time point of analysis was between two and three months of age, whereas Rehmani et al. described the onset of DCM even later than 6 months of age of mice.

On a basal level the CM specific Fto-KO (cKO) did not account for a higher mortality of FtockO mice compared to control animals. Comparison of $\mathrm{Fto}^{\mathrm{cKO}}$ and $\mathrm{Cre}^{\mathrm{Control}}$ groups demonstrated a comparable mortality. The median survival of cKO mice was 230.5 days, which is comparable to the median of 265 days in Cre ${ }^{\text {Control }}$ mice $(p=0.7)$. Flox ${ }^{\text {Control }}$ mice all survived until the last day of monitoring (day 400), proving that the loxP-sites flanking exon 3 of Fto did not affect 
survival. The higher mortality of Fto ${ }^{\mathrm{ckO}}$ and $\mathrm{Cre}^{\mathrm{Control}}$ animals is most likely a cre-recombinase mediated effect (Rehmani et al. 2019), as the Ftocko and Cre ${ }^{\text {Control }}$ groups both have crerecombinase activity. The median survival was almost comparable, and although the Ftocko mice die $\sim 30$ days earlier, it did not reach significance $(p=0.7)$. For the following experiments, only the Cre ${ }^{\text {Control }}$ group was used as controls to rule out cre-effects since no flox-mediated effect was seen.

\subsubsection{The cardiomyocyte-specific depletion of FTO in vivo leads to a worsened hypertrophic response}

To investigate a potential effect of the Fto-KO on hypertrophic growth, the previously described TAC model was applied (deAlmeida et al. 2010). Since a basal phenotype of dilatation was recognized in Fto ${ }^{\mathrm{cKO}}$ mice at the age of 4 months, it was decided to finish analysis ahead of this time point. Therefore, mice underwent TAC at the age of 8 weeks (= 2 months), and final echocardiographic analysis was performed 4 weeks after TAC when mice were 12 weeks old (3 months). By this, the basal phenotype was not likely to influence analysis. This was furthermore supported by the finding, that the cardiac performance is only slightly impaired in Fto ${ }^{\text {cKO }}$ mice compared to controls in the basal analysis by the age of 4 months (Figure 3.35), which is already one month later than the last time point of examination.

Echocardiographic analysis showed that the AwTh is increased in FtockO as well as Cre ${ }^{\text {Control }}$ mice to a comparable extent one week after TAC surgery (Figure 3.36). In Cre ${ }^{\text {Control }}$ animals this suggests a compensation to pressure overload (Norton et al. 2002), as also the LVID was unchanged. Interestingly, Fto ${ }^{\mathrm{cKO}}$ mice showed significant LV dilatation compared to Cre Control $^{\text {Con }}$ animals and a severely reduced EF. These observed changes were even stronger 4 weeks after TAC (Figure 3.37), where the EF was impaired in both groups, but more severely in FtockO mice. The Fto ${ }^{\mathrm{cKO}}$ mice showed significant LV dilatation and in Cre $\mathrm{Control}^{\mathrm{animals}}$ also tendency

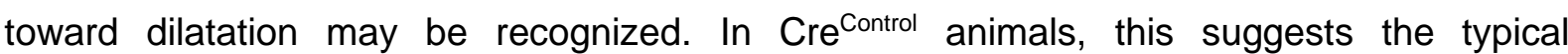
maladaptation with the heart being unable to cope with the persistent stress and ensure sufficient blood output, whereas in Fto ${ }^{\mathrm{cKO}}$ this phenotype was more severe than compared to control. Since these mice were challenged with TAC using a $27 \mathrm{G}$ needle, the constriction is rather tight which may explain the severely reduced EF already one-week post-TAC. Therefore, these analyses were additionally performed with animals that underwent TAC surgery using a 26G needle (Figure 3.38). The milder constriction with a $26 \mathrm{G}$ needle reduces mortality after TAC as well as lowers the burden exerted by the TAC surgery. This ensures that effects mediated by the KO become clearer and can be analyzed better, which is also important for future sequencing experiments. Animals that were challenged with TAC using a 26G needle, were analyzed one-week post-TAC and showed comparable but milder 
phenotypes than with a $27 \mathrm{G}$ needle (Figure 3.36, Figure 3.38). One week after 26G-TAC, a

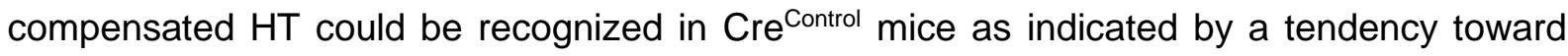
increased wall thickness, concentric decrease of LVID and a preserved EF (Figure 3.38). The Fto ${ }^{\mathrm{ckO}}$ animals in contrast showed significantly reduced EF and a tendency towards dilatation was observed with an almost unchanged wall thickness. Compared to Cre ${ }^{\text {Control }}$ animals, the LVID of Fto ${ }^{\text {cKO }}$ mice was significantly larger in the TAC group. This resembles an early DCM like heart failure phenotype with an increased heart mass and normal wall thickness at the same time. These differences in stress-response were further underlined by analysis of the LV/TL and the relative wall thickness (RWT) (Figure 3.39). In both the Fto ${ }^{\mathrm{ckO}}$ and Cre ${ }^{\text {Control }}$ mice increased LV/TL was recognized, indicating hypertrophic growth of the heart. However, the

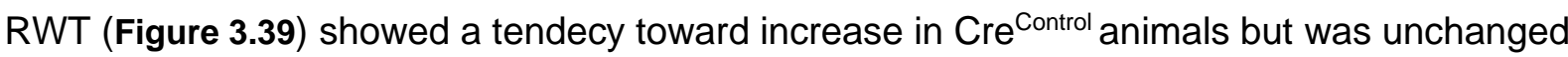
in $\mathrm{FTO}^{\mathrm{ckO}}$ mice. Thus, concentric growth may be recognized in $\mathrm{Cre}^{\mathrm{Control}}$, whereas, Fto ${ }^{\mathrm{ckO}}$ mice show a more dilatative, DCM like growth. Fto ${ }^{\text {cKO }}$ animals seem to lack a compensatory response to pressure overload. This is consistent with the early onset of dilatation of the LV in Ftocko animals and in line with the basal phenotype observed, although with a way faster progression.

Since the silencing of FTO in vitro attenuated hypertrophic growth, one may expect that this has a beneficial effect. Yet, in vivo KO of Fto lead to a worsened adaptation. The in vitro model offers limited readouts and only partial conclusions on cardiac function; therefore, in vivo experiments are necessary to reveal effects on a systems, organ, and functional level. In vivo experiments allow testing of complex hypotheses beyond just HT in CMs upon FTO-depletion. When hearts are challenged with $\mathrm{PO}$, the initial adaptation of compensated hypertrophy occurs where concentric growth of the ventricle, indicated by increased wall thickness, preserves the EF and hence the cardiac output (Toischer et al. 2010). The wall thickening derives from growth of CMs in width by incorporation of sarcomeres (Wilson et al. 2014). With persistent cardiac stress the adaptive remodeling of the heart becomes maladaptive, with longitudinal change of CM geometry and shape (Anand 2002; Norton et al. 2002; Azevedo et al. 2016), which results in LV dilatation and finally leads to heart failure. As in the Ftocko mouse an early onset of dilatation was observed with decreased EF and a tendency toward less wall thickening compared to Cre ${ }^{\text {Control }}$ animals in 26G TAC, one may conclude that the Fto-KO prevents compensated HT and thus cardiac adaptation. This is consistent with attenuated cell growth and lower ANP expression (Kessler-lcekson et al. 2002) observed in iPSC-CMs upon FTO silencing. In vitro, this seems beneficial as prevention of HT growth could prevent or attenuate transition to heart failure. Yet, it indicates the inability of the CMs to grow hypertrophic, which in vivo would be important for PO compensation but is now of maladaptive nature (Figure 4.2). 


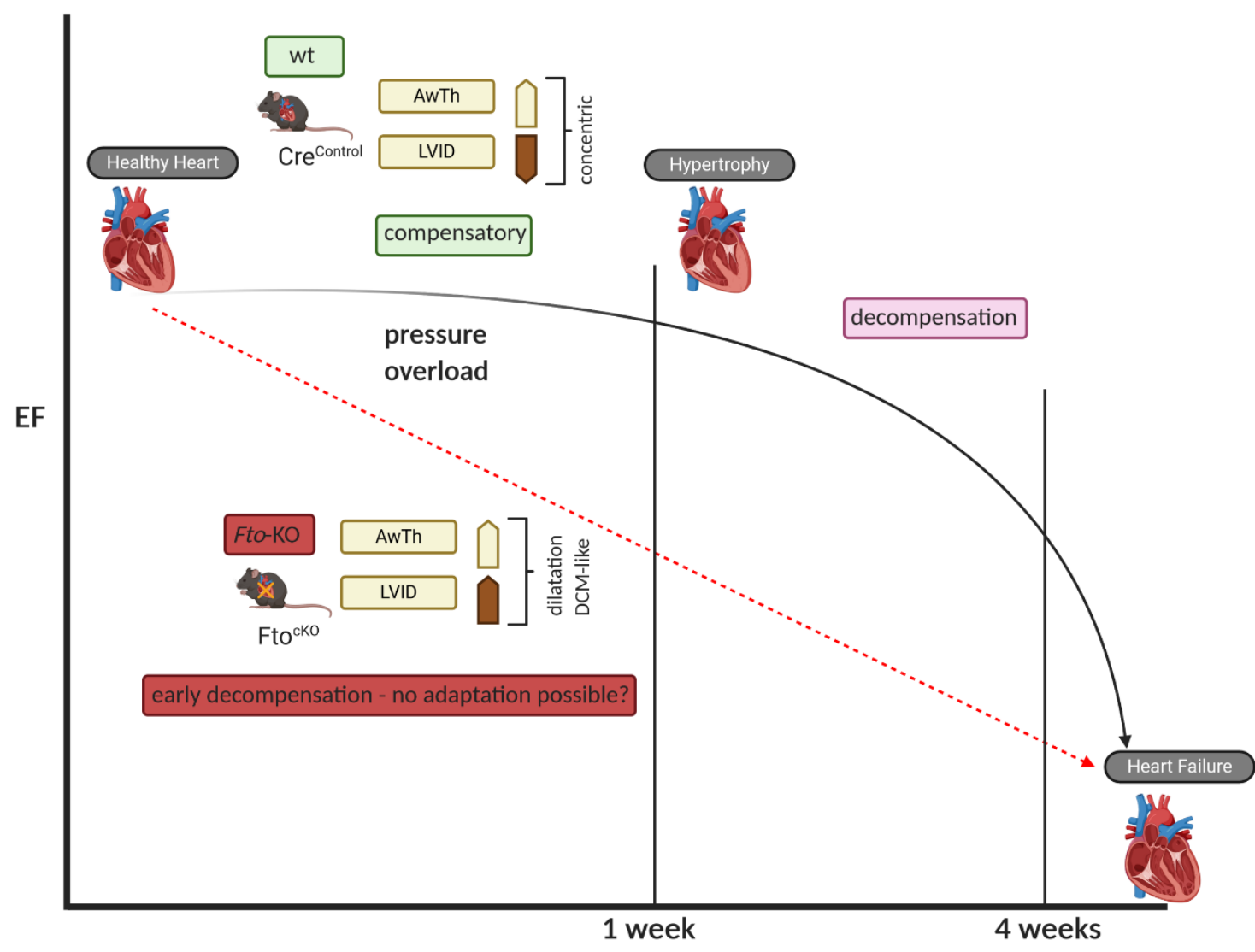

Figure 4.2 FTO depletion leads to maladaptation and early dilatation, potentially by attenuating hypertrophic growth

In response to pressure overload, FtockO present with early dilatation and a severely reduced EF. The normal, compensatory hypertrophic growth occurring one-week post-TAC with maladaptation upon persistent stress after 4 weeks seems to be omitted in Ftocko animals in comparison to Crecontrol animals. Therefore, the FTO depletion may impair the normal adaptation mechanisms of the heart, potentially by altering $\mathrm{CM}$ growth as seen in the cell culture model of attenuated hypertrophy.

FTO involvement was already discussed in cardiac defects such as arrhythmias (Carnevali et al. 2014), atrioventricular and septal defects (Boissel et al. 2009) as well as coronary artery diseases (Gustavsson et al. 2014). Unfortunately, these studies did not consider the function of FTO as a RNA demethylase, but concluded that point mutations lead to obesity and diabetes with subsequent coronary heart diseases (Gustavsson et al. 2014). Other studies reported that a global FTO-KO increased proarrhythmic remodeling (Carnevali et al. 2014) or that loss-offunction mutations lead to growth retardation of cultured fibroblasts (Boissel et al. 2009). These studies do not suggest molecular modes of action that would explain the transcriptomic changes or epitranscriptomic changes after FTO manipulation. Therefore, the underlying mechanistic processes still need to be elucidated in future research. Especially the exact influence of m6A changes after FTO depletion (via silencing or $\mathrm{KO}$ ) on various mechanisms in which m6A RNA methylation is involved remains unknown. Better insight into these molecular 
changes will be gained once MeRIP seq is performed with tissue from my in vitro as well as in vivo models.

Nevertheless, some hypotheses could already be derived from the HT and HF study with wildtype animals. A possible explanation for the lack of compensatory potential in Fto ${ }^{\text {cko }}$ mice might be a dysregulation of the global RNA methylome in their hearts. As described previously, in the wildtype animal hypomethylated transcripts were coding mainly for proteins involved in metabolism and catabolism, and hypermethylated transcripts were coding for signal transduction and structural processes. Since the RNA demethylase FTO acts as an eraser of the m6A modification, its depletion is assumed to increase global m6A methylation. Some transcripts, hypomethylated in the wildtype, basal state, may possibly get hypermethylated in Ftocko mice possibly decreasing their half-life time and accelerating their turnover. This might deregulate the essential energy conservation mechanisms usually involved in cardiac stress response leading to the inability of cells to cope with the higher demand in energy. In addition to accelerating turnover (Du et al. 2016; Ke et al. 2017), global hypermethylation may induce or influence translation of transcripts either in a reader-mediated manner (Shi H et al. 2017) or directly through ribosomal recruitment. This may affect the fraction of transcripts that were found to be hypermethylated upon cardiac stress in wildtype animals. As hypothesized earlier, these transcripts may initially be translated to a higher extent as they were enriched in polysomes. In the Fto-KO CMs, the same transcripts may be displaced or repressed by the high amount of competing previously hypomethylated transcripts, normally translated slower by only a few ribosomes. Therefore, the global methylation changes might mask or impair the otherwise tightly orchestrated methylation-dependent translational 'boost' of important signal transduction genes in response to stress.

As the hypermethylated transcripts from the wildtype mouse study coded for signal transduction, the cells signaling in response to TAC may be impaired in Ftocko hearts, further worsening the adaptation. Moreover, some of the hypermethylated transcripts enriched in the polysome in the wildtype study were enriched in GO terms of proliferation and structural changes. The deregulation of such transcripts may additionally impair the remaining compensatory potential in Fto ${ }^{\mathrm{cKO}}$ mice.

Another possible effect exerted by $\mathrm{m} 6 \mathrm{~A}$ methylation change on the maladaptive phenotype observed in KO mice may be that the cell shape and geometry of CMs is affected. The hypothesis that altered $\mathrm{m} 6 \mathrm{~A}$ levels affects $\mathrm{CM}$ geometry was already postulated and shown by two other studies (Dorn et al. 2019; Kmietczyk et al. 2019). Kmietzyck and colleagues showed a similar effect as seen in my study where phenylephrine (PE) induced cell hypertrophy was blunted upon Fto-KO in neonatal rat cardiomyocytes (NRCM) (Kmietczyk et al. 2019). Furthermore, the group demonstrated that the cell size increases and that the typical 
hypertrophy markers Nppa (the gene coding for ANP) and Nppb (coding for BNP) expression was induced upon Mett/3- KO. FTO depletion should increase global m6A methylation whereas METTL3-KO should decrease m6A levels. Consistent with my observations in FTOknockdown iPSC-CMs, Kmietczyk and colleagues showed that lower m6A levels increased cell size whereas m6A increase attenuated cell growth. Interestingly, Dorn et al. show quite an opposite result, where the $\mathrm{KO}$ of Mett/3 and hence $\mathrm{m} 6 \mathrm{~A}$ decrease in mice reduced the crosssectional area of isolated CMs (Dorn et al. 2019). These contradictious findings may be explained by the differences in model organisms (mice vs. rat CMs vs. iPSC-CMs) or by methodical differences of the used approaches (siRNA, Adenovirus, AAV or cre-lox). Furthermore, concerning the shape and size of iPSC-CMs it must also be considered that they do not resemble isolated CMs in shape. iPSC-CM are of circular shape, whereas native adult CMs are of a more rectangular shape being bigger in length than width (Toischer et al. 2010), hence not allowing conclusions on geometrical growth differences.

These findings of different growth and response mechanisms of CMs in different models underlines the diversity of epigenetically mediated effects and the complex role of m6A RNA methylation. Manipulation of the RNA methylation machinery may increase the number of affected processes even more (due to global dysregulation). In order to understand the specificity and efficiency of epigenetic effects, a time dependent manipulation of the RNA methylation should be investigated. The here presented differences in the response to cardiac stress in either iPSC-CMs or mice might be explained by differences in timing and duration of FTO depletion. Where in the iPSC-CMs FTO is silenced only transiently and shortly before hypertrophic induction, in the Fto ${ }^{\mathrm{cKO}}$ mouse model FTO is constantly depleted, so that exerted effects are present all the time. So potentially a short-term inhibition of FTO at specific states of disease progression and resulting epigenetic changes may be protective, whereas constant inhibition by for example $\mathrm{KO}$ could lead to maladaptive responses by prevention of adaptive remodeling. If this holds true, temporal manipulation of the RNA methylome could be tested as a potential therapeutic approach in heart failure. Hypothetically, an FTO-inhibition during or after an adaptive remodeling in the critical phase of $\mathrm{PO}$ might prevent maladaptive or excessive growth, hence, attenuating the progression to HF. It would be interesting to test this approach using an inducible $\mathrm{KO}$ mouse model like the aMHC-MerCreMer mouse line. Here, the crerecombinase is inactive until Tamoxifen application. This would allow a Fto-KO to be induced sometime after induction of HT by TAC surgery, so one could observe the response to cardiac stress upon time-dependent FTO manipulation. Another possibility is the use of FTO-inhibitors. First FTO-inhibitors were described (Peng et al. 2019) and already applied intraperitoneal in mice with their safety for in vivo usage proven. These inhibitors could be applied to mice at some time point after TAC when hearts already initially adapted to PO, to check if further disease progression is attenuated. Another possibility to investigate the effect of FTO 
manipulation on hypertrophic response and disease progression could be overexpression (OE) studies. As the Fto-KO presented maladaptive, an OE of Fto after TAC with for example adenoassociated virus (AAV) may be beneficial. Even more, AAVs with a cardiac specific serotype could be used to induce a $\mathrm{CM}$ specific $\mathrm{OE}$.

Concerning the maladaptive responses described for $\mathrm{Fto}^{\mathrm{cKO}}$ mice earlier in this section, it is possible that the observed effects exerted by the Fto-KO may be mediated by $\mathrm{m} 6 \mathrm{Am}$ changes rather than m6A changes. FTO demethylates m6Am not only on mRNA, but especially on snRNAs, which were shown to mediate splicing events (Bartosovic et al. 2017; Mauer et al. 2019). This could also impact transcriptional regulation. Another mechanism could be that m6Am marks in the 5' UTR can lead to cap-independent translation (Meyer et al. 2015). These processes may influence transcriptional as well as translational regulation resulting in the maladaptation of CMs. This is a problem that needs to be considered in further experiments, which could be rather difficult due to the inability of antibodies to discriminate between $\mathrm{m} 6 \mathrm{~A}$ and m6Am (Zaccara et al. 2019).

\subsection{Summary and Outlook}

In this study I could show that m6A RNA methylation is present to a considerable amount in mouse and human heart tissue and furthermore is altered in cardiac HT and HF. A few interesting observations were made throughout the study. Most importantly, methylation changes in response to cardiac stress outnumber expression changes by far, underlining the importance of epigenetic changes in cardiac diseases. Only a minor number of transcripts was detected to be affected on the methylation and expression level at the same time. Therefore, the often-hypothesized effect of m6A methylation on RNA stability and decay, affecting mRNA levels directly, may not be the main mechanism in case of cardiac HT and HF. I postulated the hypothesis, that indeed m6A marks may affect mRNA stability, but more likely by increasing half-life time of specific transcripts participating in processes continuously needed in cell stress, such as energy conservation mechanisms. This mechanism reduces the need of cells to continuously transcribe such mRNAs in the nucleus and to transport those mRNAs throughout the cell, which is beneficial for CMs since they are relatively large in size. Furthermore, I hypothesized that hypermethylation marks transcripts for a shorter half-life time, but for an increased translation at the same time. This fits quite well to the observation, that hypermethylated transcripts code for signal transduction processes, and with this simultaneous turnover and high translation the cellular signal transduction response can be regulated dynamically and fast. Hence, I postulated a fast response mechanism in cardiac tissues that m6A methylation may regulate. These findings lead to the conclusion, that $\mathrm{m} 6 \mathrm{~A}$ methylation in response to cardiac $\mathrm{HT}$ and $\mathrm{HF}$ mainly regulates translation. This was confirmed by the finding 
that the m6A methylation level positively correlated with ribosomal occupancy, hence influencing protein levels. The effect of RNA methylation on the protein level was furthermore proven for CALM1, whose transcript is unchanged at the expression level. Therefore, a potential new mechanism of transcription-independent translational regulation by $\mathrm{m} 6 \mathrm{~A}$ methylation was hypothesized. Future studies are needed to prove this mechanism for other targets and to elucidate how this control mechanism works and if $\mathrm{m} 6 \mathrm{~A}$ methylation influences rather mRNA localization, the tertiary structure ('m6A-switch') or if translation regulation is mainly reader mediated. For example, RBP mapping could be applied by UV-crosslinking to fix RBPs to RNA and then compare the RBP landscape with the methylation landscape, to gain better insights in how m6A methylation patterns affect reader binding. Moreover, miRNA mediated translational control should be investigated as additional mechanism in the future. This could be done with MeRIP-seq of miRNAs. Thereby, it could be investigated how miRNAs participate in mRNA regulation as well as how m6A methylation of miRNAs themselves regulates the RISC pathway and affects miRNA-mRNA interaction.

Additionally, I gave first insights on a potential therapeutic application of FTO manipulation for treatment of HF by investigating effects after silencing of the demethylase FTO in vitro, as well as by CM specific knockout in vivo. Interestingly, the potentially beneficial anti-hypertrophic effect of FTO depletion in iPSC-CM culture was shown to be detrimental in vivo. It showed that the attenuated hypertrophic response in vitro rather prevents adaptive remodeling in vivo. This is possibly caused by the global deregulation of altered m6A levels affecting cell shape or geometry, or by regulating translation. If FTO depletion and therefore increased m6A levels promotes longitudinal growth of CMs instead of growth in width should be investigated in future experiments. This effect could for example be investigated by immunofluorescence staining of isolated mouse CMs from Fto ${ }^{\mathrm{cKO}}$ mice or by WGA stain and subsequent measurement of crosssectional areas. Furthermore, whether FTO manipulation affects the m6A or rather m6Am level or both still needs to be addressed in future experiments. Also, iPSC-CM cell culture needs to be expanded to collect a sufficient amount of RNA to perform the measurement of $\mathrm{m} 6 \mathrm{~A}$ or m6Am level in the FTO-knockdown cells in vitro.

As a next step, MeRIP-Seq should be performed with LV tissues of healthy (Sham) Fto ${ }^{\mathrm{cKO}}$ mice as well as of Fto ${ }^{\mathrm{cKO}}$ mice challenged with TAC. This could help to elucidate the transcriptomic and epitranscriptomic changes of these mice on basal level as well as in disease response, similar to the experiments performed with wildtype mice in this study. It would indirectly reveal if FTO affects m6A or m6Am in this model and would omit the bias of unspecific m6A antibodies also detecting m6Am. Enrichment of methylation changes in 5' UTR would point to m6Am being mainly affected, since this modification is mainly found to occur there (Linder et al. 2015). Robust methylation changes in other parts of transcripts would indicate that also the m6A 
marks are affected which were found to be enriched in the CDS and 3' UTR (Meyer et al. 2012).

Moreover, it would be interesting to apply other cardiac disease models to the Fto ${ }^{\text {ckO }}$ mice like for example volume overload induced by aortocaval shunt surgery. Thereby, one could examine if the potential longitudinal growth forced by FTO depletion worsens the dilatative response occurring in response to volume overload even more or if a completely different phenotype occurs.

\subsection{Conclusion}

In conclusion, m6A RNA methylation is a conserved and important mechanism in cardiac tissue that changes in response to stress and during progression to heart failure. The processes affected by differential RNA methylation are substantially different from those affected by differential expression. Altered $\mathrm{m} 6 \mathrm{~A}$ levels lead to translational regulation even for transcripts unchanged at the expression level. Depletion of the RNA demethylase FTO from CMs leads to reduced growth and impaired adaptation and a faster progression to heart failure. Overall, these results indicate the importance of the epitranscriptome in cardiac diseases and makes it an interesting target for therapeutic interventions by for example modulating hypertrophic responses in a time-dependent manner potentially mediated by $\mathrm{m} 6 \mathrm{~A}$ methylation. 


\section{Appendix}

Table 5.1 Patient Data of healthy, non-failing tissue samples

\begin{tabular}{|c|c|c|c|c|}
\hline $\begin{array}{c}\text { Patient } \\
\text { Sample ID }\end{array}$ & \multicolumn{2}{|c|}{$\begin{array}{c}\text { Type of } \\
\text { Cardiomyopathy Sex }\end{array}$} & Age [yrs] & EF [\%] \\
\hline NF2 & NF & m & 74 & 50 \\
\hline NF3 & NF & $f$ & 60 & 50 \\
\hline NF4 & NF & $f$ & 62 & 50 \\
\hline NF5 & NF & $m$ & 59 & 60 \\
\hline NF6 & NF & $m$ & 60 & 69 \\
\hline
\end{tabular}

Table 5.2 Patient Data of end-stage HF tissue samples

\begin{tabular}{|c|c|c|c|c|c|c|c|c|c|c|c|c|c|c|}
\hline $\begin{array}{c}\text { Patient } \\
\text { Sample No. }\end{array}$ & $\begin{array}{l}\text { Type of } \\
\text { Cardiomyopathy }\end{array}$ & Sex & Age & EF & Lvedd & ACE Inhibitor & Betablocker & Diuretics & Digoxin & $\begin{array}{c}\text { Catecholami } \\
\text { ne }\end{array}$ & Amiodarone & $\begin{array}{c}\text { AT1- } \\
\text { Receptor } \\
\text { antagonist }\end{array}$ & $\begin{array}{l}\text { Aldosterone } \\
\text { Antagonist }\end{array}$ & $\begin{array}{c}\text { PDE- } \\
\text { Inhibitor }\end{array}$ \\
\hline 585 & ICM & $f$ & 59 & 20 & 70 & & $x$ & $x$ & & & & & & $x$ \\
\hline 589 & DCM & $\mathrm{m}$ & 40 & 15 & 63 & $x$ & $x$ & $x$ & $x$ & & $x$ & & $x$ & \\
\hline 702 & DCM & f & 62 & 22 & 83 & $x$ & $x$ & $x$ & & $x$ & $x$ & & $x$ & \\
\hline 723 & $\mathrm{DCM}$ & $\mathrm{m}$ & 49 & 20 & 69 & & $x$ & $x$ & & & & & $x$ & \\
\hline
\end{tabular}


A
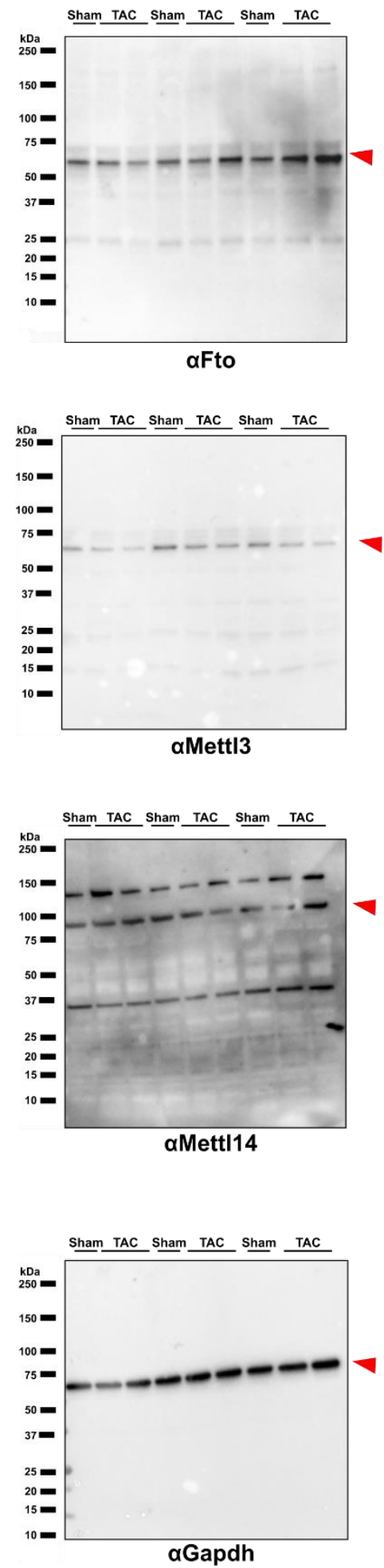

B $\underline{N F} \quad \underline{H F} \quad \underline{N F} \quad \underline{H F} \quad N \quad \underline{N F} \quad \underline{N F} \quad \underline{H F} \quad \underline{N F} \quad \underline{H F} \quad \underline{N F} \quad \underline{H F}$
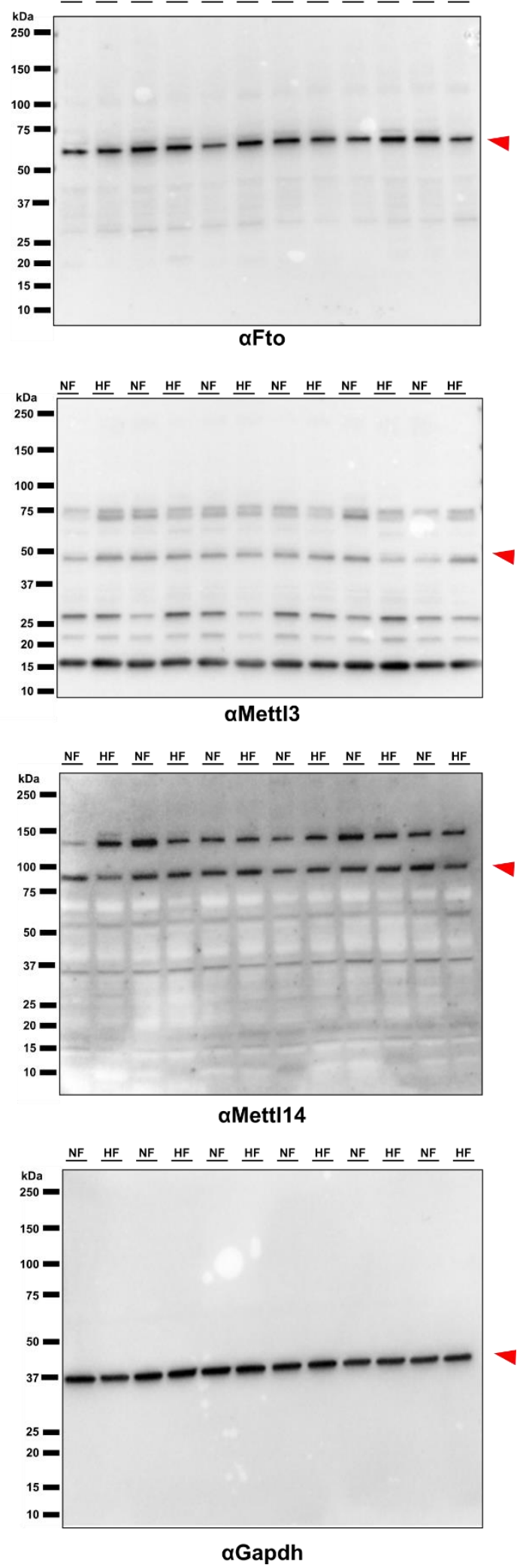

Figure 5.1 Full blots of the methylation machinery in mouse and human LV tissue

Representative blots of the m6A RNA methylation writers METTL3 and METTL14 as well as the RNA demethylase FTO are shown. (A) Western blot images of mouse samples are shown. The expected protein bands are indicated with arrows. With the METTL14 antibody, unspecific protein bands of unknown origin occurred at $\sim 35 \mathrm{kDa}$ and $\sim 130 \mathrm{kDa}$. Each lane represents an independent animal serving as biological replicate with $n=3$ for Sham and $n=6$ for TAC. (B) Protein blots of human tissue lysates are shown and expected bands indicated. For METTL3, many unspecific bands 
occurred at different sizes with unknown origin. A band of unknown origin appeared with METTL14 antibody at $\sim 130 \mathrm{kDa}$. Each lane represents an independent biological replicate obtained from tissues from different patients. $\mathrm{n}=6$ for NF and HF.

A
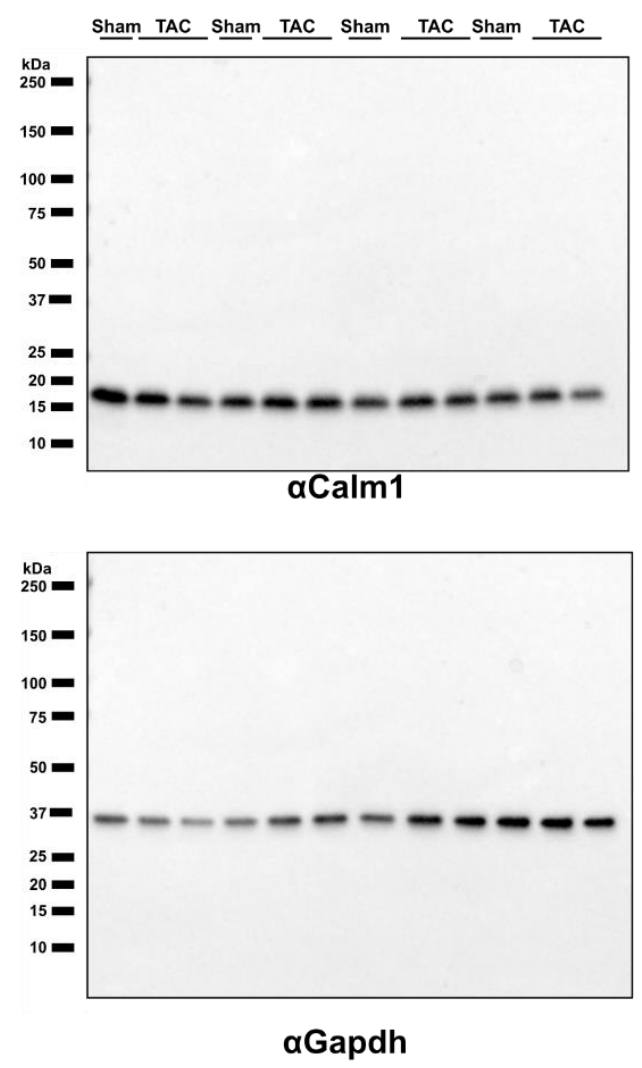

B
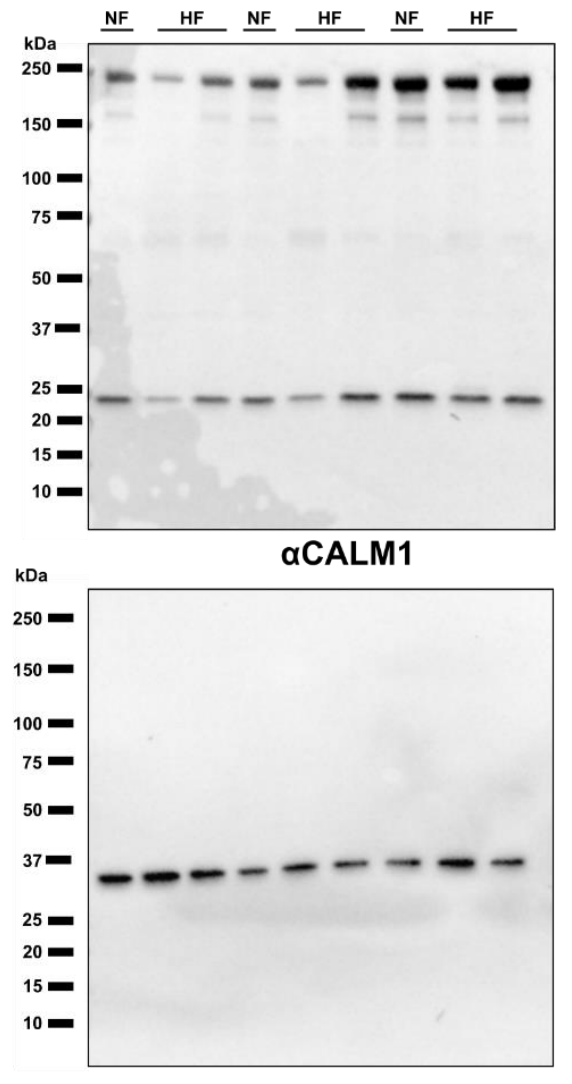

aGAPDH

Figure 5.2 Fill blots Calmodulin1 Mouse and Human

Representative blots of CALM1 are presented. (A) Western blot images of mouse samples are shown. The expected specific protein band at $\sim 17 \mathrm{kDa}$ was obtained for CALM1 and the respective GAPDH blot is shown. Each lane represents an independent animal serving as biological replicate with $n=4$ for Sham and $n=8$ for TAC. (B) Protein blots of human tissue lysates are shown and the CALM1 band is seen at $\sim 17 \mathrm{kDa}$. Unspecific protein bands at $\sim 250 \mathrm{kDa}$ with unknown origin occurred. The respective GAPDH blot used for normalization is presented. Each lane represents an independent biological replicate obtained from tissues from different patients. $n=3$ for NF and $n=6$ for HF. 

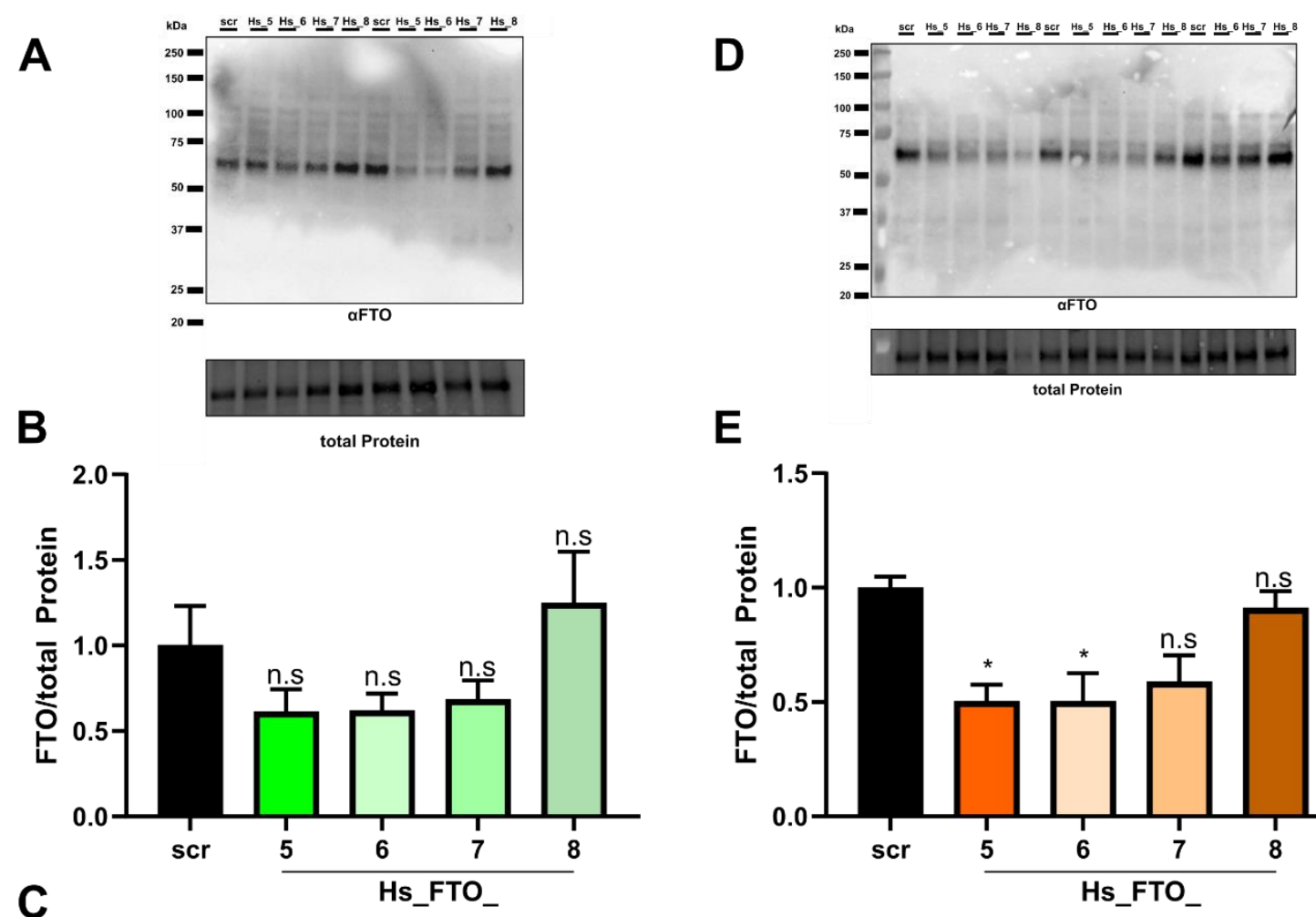

E
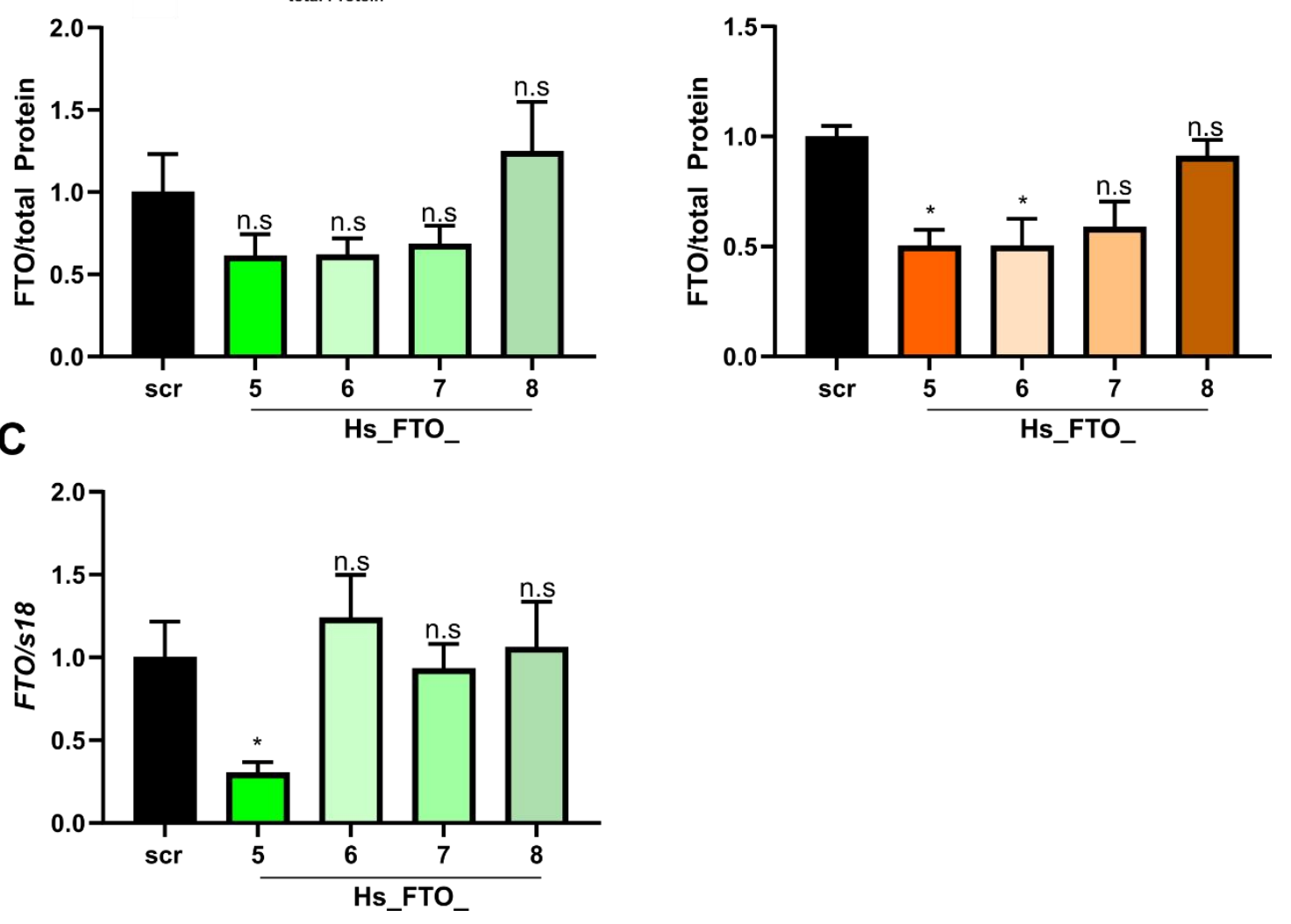

Figure 5.3 Testing different siRNAs targeting FTO-mRNA for effectivity

Four different siRNAs, each binding to a different region of the FTO-mRNA were tested for best performance. Effectivity was tested after 48 hours of transfection time in both cell lines. PCTRL (A-C) and WT (D, E). (A, B): Western blotting showed reduction of FTO protein upon Hs_FTO_5-7 siRNA transfection in PCTRL cells. Cells from one differentiation were used, with different treated wells as biological replicates ( $n=4$ for scramble, Hs_FTO_5-7, $n=3$ for Hs_FTO_8). (C) RT-qPCR analysis revealed significant reduction of FTO-mRNA after Hs_FTO_5 transfection in pCTRL cells. The other siRNAs seem to be not effective. Cells from one differentiation were used, with different treated wells as biological replicates ( $\mathrm{n}=4$ for scramble, Hs_FTO_5-7, $\mathrm{n}=2$ for Hs_FTO_8) (D, E) In WT cells, Western blotting demonstrated a no significant reduction of FTO protein level, but a decrease can be appreciated upon Hs_FTO_5 and_6 transfection. Hs_FTO-8 siRNA lead to no protein level change at all. Cells from one differentiation were used, with different treated wells as biological replicates ( $\mathrm{n}$ = 3 for scramble, Hs_FTO_6-8, $n=2$ for Hs_FTO_5). (B, C, E) changes in protein, as well as RNA level were normalized and compared to scramble controls and mean with SEM is shown. t-test with Welch's correction was performed. Significance levels are represented on top of each bar. 


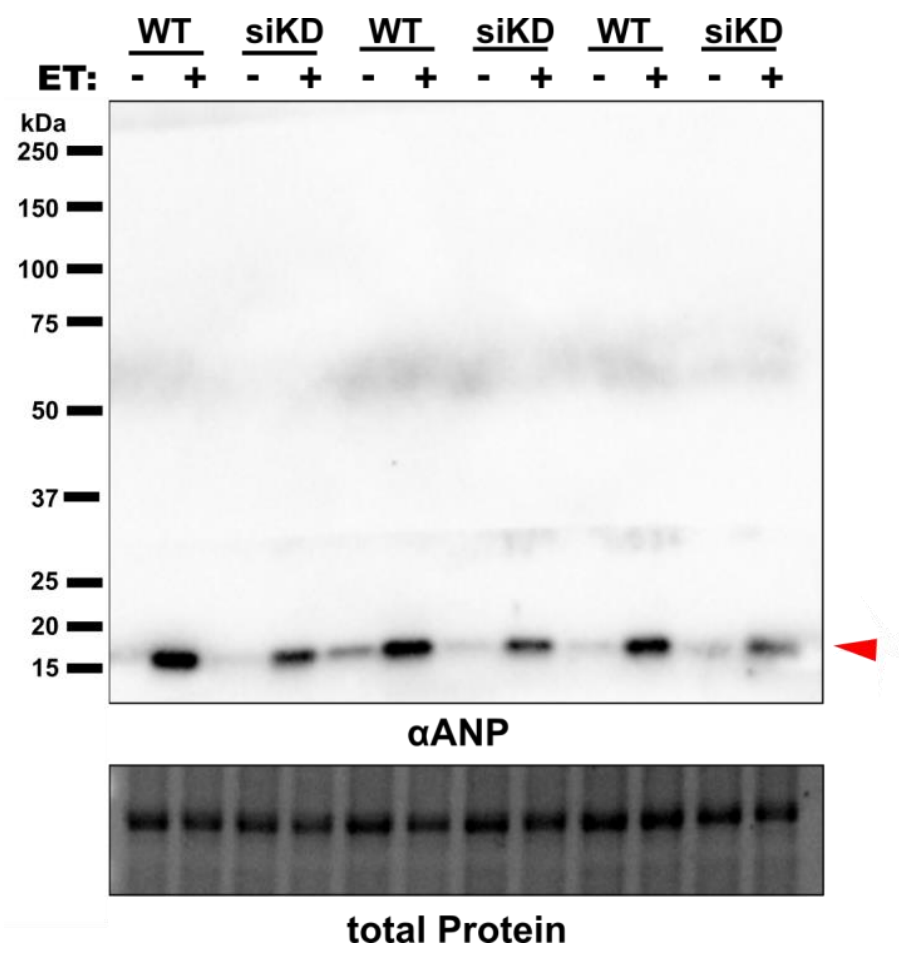

Figure 5.4 Full blot ANP

Since a strong signal for an unspecific band of unknown origin was detected with ANP antibody, decreasing sensitivity of the chemiluminescence signal, the unspecific band was covered, and blots imaged. This led to a more prominent ANP specific band from which the quantified lower ANP protein level in FTOsikD cells upon ET-1 stimulation compared to FTOWT cells could also visibly be recognized.

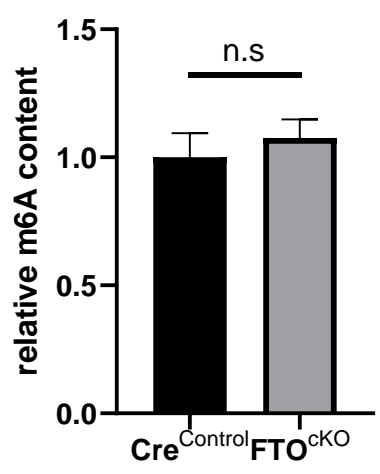

Figure $5.5 \mathrm{~m} 6 \mathrm{~A}$ Assay did not reveal changed m6A levels in Fto ${ }^{\mathrm{cko}}$ mice compared to Cre Control

Relative m6A level analysis by an $\mathrm{m} 6 \mathrm{~A}$ assay did not reveal changes of $\mathrm{m} 6 \mathrm{~A}$ abundance in rRNA depleted RNA isolated from FtockO in comparison to Cre ${ }^{\text {Control }}$. t-test with Welch's correction was performed with $\mathrm{n}=4$ for $\mathrm{Cre}^{\mathrm{Control}}$ and Fto ${ }^{\mathrm{cKO}}$. 


\section{Bibliography}

Adiarto S, Heiden S, Vignon-Zellweger N, Nakayama K, Yagi K, Yanagisawa M, Emoto N (2012): ET-1 from endothelial cells is required for complete angiotensin II-induced cardiac fibrosis and hypertrophy. Life Sciences $\underline{91}$, 651-657

Agah R, Frenkel PA, French BA, Michael LH, Overbeek PA, Schneider MD (1997): Gene recombination in postmitotic cells. Targeted expression of Cre recombinase provokes cardiac-restricted, site-specific rearrangement in adult ventricular muscle in vivo. J Clin Invest $\underline{100}, 169-179$

Alarcón CR, Goodarzi H, Lee H, Liu X, Tavazoie S, Tavazoie SF (2015): HNRNPA2B1 Is a Mediator of m6A-Dependent Nuclear RNA Processing Events. Cell 162, 1299-1308

Ames EG, Lawson MJ, Mackey AJ, Holmes JW (2013): Sequencing of mRNA identifies reexpression of fetal splice variants in cardiac hypertrophy. Journal of Molecular and Cellular Cardiology $\underline{62}, 99-107$

Anand IS (2002): Ventricular remodeling without cellular contractile dysfunction. Journal of Cardiac Failure $\underline{8}$, S401-S408

Anand P, Brown JD, Lin CY, Qi J, Zhang R, Artero PC, Alaiti MA, Bullard J, Alazem K, Margulies KB, et al. (2013): BET bromodomains mediate transcriptional pause release in heart failure. Cell $\underline{154}, 569-582$

Anversa P, Olivetti G, Melissari M, Loud AV (1980): Stereological measurement of cellular and subcellular hypertrophy and hyperplasia in the papillary muscle of adult rat. J Mol Cell Cardiol $\underline{12}, 781-795$

Archer CR, Robinson EL, Drawnel FM, Roderick HL (2017): Endothelin-1 promotes hypertrophic remodelling of cardiac myocytes by activating sustained signalling and transcription downstream of endothelin type A receptors. Cellular Signalling $\underline{36}, 240$ 254

Azevedo PS, Polegato BF, Minicucci MF, Paiva SAR, Zornoff LAM (2016): Cardiac Remodeling: Concepts, Clinical Impact, Pathophysiological Mechanisms and Pharmacologic Treatment. Arquivos Brasileiros de Cardiologia

Backs J, Olson EN (2006): Control of cardiac growth by histone acetylation/deacetylation. Circ Res $\underline{98}, 15-24$

Bailey AS, Batista PJ, Gold RS, Chen YG, de Rooij DG, Chang HY, Fuller MT (2017): The conserved RNA helicase YTHDC2 regulates the transition from proliferation to differentiation in the germline. eLife $\underline{6}$, e26116

Banerjee I, Fuseler JW, Price RL, Borg TK, Baudino TA (2007): Determination of cell types and numbers during cardiac development in the neonatal and adult rat and mouse. Am J Physiol Heart Circ Physiol 293, H1883-1891

Barbieri I, Tzelepis K, Pandolfini L, Shi J, Millán-Zambrano G, Robson SC, Aspris D, Migliori V, Bannister AJ, Han N, et al. (2017): Promoter-bound METTL3 maintains myeloid leukaemia by m6A-dependent translation control. Nature 552, 126-131

Bartosovic M, Molares HC, Gregorova P, Hrossova D, Kudla G, Vanacova S (2017): N6methyladenosine demethylase FTO targets pre-mRNAs and regulates alternative splicing and 3'-end processing. Nucleic Acids Res $\underline{45}, 11356-11370$ 
Batista PJ, Molinie B, Wang J, Qu K, Zhang J, Li L, Bouley DM, Lujan E, Haddad B, Daneshvar K, et al. (2014): m6A RNA Modification Controls Cell Fate Transition in Mammalian Embryonic Stem Cells. Cell Stem Cell 15, 707-719

Benjamin EJ, Virani SS, Callaway CW, Chamberlain AM, Chang AR, Cheng S, Chiuve SE, Cushman M, Delling FN, Deo R, et al. (2018): Heart Disease and Stroke Statistics2018 Update: A Report From the American Heart Association. Circulation 137

Bertero A, Brown S, Madrigal P, Osnato A, Ortmann D, Yiangou L, Kadiwala J, Hubner NC, de los Mozos IR, Sadée C, et al. (2018): The SMAD2/3 interactome reveals that TGF $\beta$ controls m6A mRNA methylation in pluripotency. Nature $\underline{555}, 256-259$

Berulava T, Rahmann S, Rademacher K, Klein-Hitpass L, Horsthemke B (2015a): N6Adenosine Methylation in MiRNAs. PLoS ONE 10, e0118438

Berulava T, Rahmann S, Rademacher K, Klein-Hitpass L, Horsthemke B (2015b): N6Adenosine Methylation in MiRNAs. PLOS ONE $\underline{10}$, e0118438

Berulava T, Buchholz E, Elerdashvili V, Pena T, Islam MR, Lbik D, Mohamed BA, Renner A, Lewinski D, Sacherer M, et al. (2020): Changes in m6A RNA methylation contribute to heart failure progression by modulating translation. Eur J Heart Fail 22, 54-66

Bhattacharyya SN, Habermacher R, Martine U, Closs El, Filipowicz W (2006): Relief of microRNA-mediated translational repression in human cells subjected to stress. Cell $\underline{125}, 1111-1124$

Bindea G, Mlecnik B, Hackl H, Charoentong P, Tosolini M, Kirilovsky A, Fridman W-H, Pagès F, Trajanoski Z, Galon J (2009): ClueGO: a Cytoscape plug-in to decipher functionally grouped gene ontology and pathway annotation networks. Bioinformatics $\underline{25}$, 10911093

Blanco S, Dietmann S, Flores JV, Hussain S, Kutter C, Humphreys P, Lukk M, Lombard P, Treps L, Popis M, et al. (2014): Aberrant methylation of t RNA s links cellular stress to neuro-developmental disorders. EMBO J $\underline{33}, 2020-2039$

Boczek NJ, Gomez-Hurtado N, Ye D, Calvert ML, Tester DJ, Kryshtal DO, Hwang HS, Johnson CN, Chazin WJ, Loporcaro CG, et al. (2016): Spectrum and Prevalence of CALM1 -, CALM2 -, and CALM3 -Encoded Calmodulin Variants in Long QT Syndrome and Functional Characterization of a Novel Long QT Syndrome-Associated Calmodulin Missense Variant, E141G. Circ Cardiovasc Genet $\underline{9}$, 136-146

Bodi Z, Bottley A, Archer N, May ST, Fray RG (2015): Yeast m6A Methylated mRNAs Are Enriched on Translating Ribosomes during Meiosis, and under Rapamycin Treatment. PLoS One $\underline{10}$

Boissel S, Reish O, Proulx K, Kawagoe-Takaki H, Sedgwick B, Yeo GSH, Meyre D, Golzio C, Molinari F, Kadhom N, et al. (2009): Loss-of-Function Mutation in the DioxygenaseEncoding FTO Gene Causes Severe Growth Retardation and Multiple Malformations. The American Journal of Human Genetics $\underline{85}$, 106-111

Bokar JA, Shambaugh ME, Polayes D, Matera AG, Rottman FM (1997): Purification and cDNA cloning of the AdoMet-binding subunit of the human mRNA (N6-adenosine)methyltransferase. RNA $\underline{3}, 1233-1247$

Borchert T, Hübscher D, Guessoum CI, Lam T-DD, Ghadri JR, Schellinger IN, Tiburcy M, Liaw NY, Li Y, Haas J, et al. (2017): Catecholamine-Dependent $\beta$-Adrenergic Signaling in a 
Pluripotent Stem Cell Model of Takotsubo Cardiomyopathy. Journal of the American College of Cardiology $\underline{70}$, 975-991

Brencicova E, Diebold SS (2013): Nucleic acids and endosomal pattern recognition: how to tell friend from foe? Front Cell Infect Microbiol $\underline{3}$

Brengues M, Teixeira D, Parker R (2005): Movement of eukaryotic mRNAs between polysomes and cytoplasmic processing bodies. Science $\underline{310}, 486-489$

Brundel BJJM, Van Gelder IC, Henning RH, Tuinenburg AE, Wietses M, Grandjean JG, Wilde AAM, Van Gilst WH, Crijns HJGM (2001): Alterations in potassium channel gene expression in atria of patients with persistent and paroxysmal atrial fibrillation: differential regulation of protein and mRNA levels for $\mathrm{K}+$ channels. Journal of the American College of Cardiology 37, 926-932

Buchan JR, Parker R (2009): Eukaryotic Stress Granules: The Ins and Outs of Translation. Molecular Cell $\underline{36}$, 932-941

Bupha-Intr T, Haizlip KM, Janssen PML (2012): Role of Endothelin in the Induction of Cardiac Hypertrophy In Vitro. PLoS ONE $\underline{7}$, e43179

Burchfield JS, Xie M, Hill JA (2013): Pathological Ventricular Remodeling: Mechanisms: Part 1 of 2 . Circulation $\underline{128}, 388-400$

Carnevali L, Graiani G, Rossi S, Al Banchaabouchi M, Macchi E, Quaini F, Rosenthal N, Sgoifo A (2014): Signs of cardiac autonomic imbalance and proarrhythmic remodeling in FTO deficient mice. PLoS ONE $\underline{9}$, e95499

Carthew RW, Sontheimer EJ (2009): Origins and Mechanisms of miRNAs and siRNAs. Cell $136,642-655$

Chang M, Lv H, Zhang W, Ma C, He X, Zhao S, Zhang Z-W, Zeng Y-X, Song S, Niu Y, Tong W-M (2017): Region-specific RNA m6A methylation represents a new layer of control in the gene regulatory network in the mouse brain. Open Biol $\underline{7}$

Chassé H, Boulben S, Costache V, Cormier P, Morales J (2017): Analysis of translation using polysome profiling. Nucleic Acids Res $\underline{45}$, e15

Chaterji S, Ahn EH, Kim D-H (2017): CRISPR Genome Engineering for Human Pluripotent Stem Cell Research. Theranostics $\underline{7}, 4445-4469$

Chen B, Li Y, Song R, Xue C, Xu F (2019): Functions of RNA N6-methyladenosine modification in cancer progression. Molecular Biology Reports $\underline{46}$, 2567-2575

Choe J, Lin S, Zhang W, Liu Q, Wang L, Ramirez-Moya J, Du P, Kim W, Tang S, Sliz P, et al. (2018): mRNA circularization by METTL3-elF3h enhances translation and promotes oncogenesis. Nature $\underline{561}, 556-560$

Choi J, leong K-W, Demirci H, Chen J, Petrov A, Prabhakar A, O'Leary SE, Dominissini D, Rechavi G, Soltis SM, et al. (2016): N6-methyladenosine in mRNA disrupts tRNA selection and translation elongation dynamics. Nat Struct Mol Biol 23, 110-115

Cui X, Meng J, Zhang S, Chen Y, Huang $Y$ (2016): A novel algorithm for calling mRNA $m{ }^{6} A$ peaks by modeling biological variances in MeRIP-seq data. Bioinformatics $\underline{32}$, i378i385 
de Bruin RG, Rabelink TJ, van Zonneveld AJ, van der Veer EP (2017): Emerging roles for RNA-binding proteins as effectors and regulators of cardiovascular disease. Eur Heart J ehw567

de Nadal E, Ammerer G, Posas F (2011): Controlling gene expression in response to stress. Nat Rev Genet 12, 833-845

deAlmeida AC, van Oort RJ, Wehrens XHT (2010): Transverse Aortic Constriction in Mice. JoVE 1729

Desrosiers R, Friderici K, Rottman F (1974): Identification of Methylated Nucleosides in Messenger RNA from Novikoff Hepatoma Cells. Proceedings of the National Academy of Sciences $\underline{71}, 3971-3975$

Dominissini D, Moshitch-Moshkovitz S, Schwartz S, Salmon-Divon M, Ungar L, Osenberg S, Cesarkas K, Jacob-Hirsch J, Amariglio N, Kupiec M, et al. (2012): Topology of the human and mouse m6A RNA methylomes revealed by m6A-seq. Nature $\underline{485}, 201-206$

Dorn LE, Lasman L, Chen J, Xu X, Hund TJ, Medvedovic M, Hanna JH, van Berlo JH, Accornero $\mathrm{F}$ (2019): The $\mathrm{N}^{6}$-Methyladenosine mRNA Methylase METTL3 Controls Cardiac Homeostasis and Hypertrophy. Circulation 139, 533-545

Du H, Zhao Y, He J, Zhang Y, Xi H, Liu M, Ma J, Wu L (2016): YTHDF2 destabilizes $\mathrm{m}^{6} \mathrm{~A}$ containing RNA through direct recruitment of the CCR4-NOT deadenylase complex. Nature Communications $\underline{7}, 12626$

Du Y, Hou G, Zhang H, Dou J, He J, Guo Y, Li L, Chen R, Wang Y, Deng R, et al. (2018): SUMOylation of the m6A-RNA methyltransferase METTL3 modulates its function. Nucleic Acids Research 46, 5195-5208

Edupuganti RR, Geiger S, Lindeboom RGH, Shi H, Hsu PJ, Lu Z, Wang S-Y, Baltissen MPA, Jansen PWTC, Rossa M, et al. (2017): N6-methyladenosine (m6A) recruits and repels proteins to regulate mRNA homeostasis. Nat Struct Mol Biol 24, 870-878

Engel M, Eggert C, Kaplick PM, Eder M, Röh S, Tietze L, Namendorf C, Arloth J, Weber P, Rex-Haffner M, et al. (2018): The Role of m6A/m-RNA Methylation in Stress Response Regulation. Neuron $\underline{99}$, 389-403.e9

Fasken MB, Corbett AH (2009): Mechanisms of nuclear mRNA quality control. RNA Biology $\underline{6}$, 237-241

Fazi F, Fatica A (2019): Interplay Between N6-Methyladenosine (m6A) and Non-coding RNAs in Cell Development and Cancer. Front Cell Dev Biol $\underline{7}, 116$

Feng Y, Chen H, Cai J, Zou L, Yan D, Xu G, Li D, Chao W (2015): Cardiac RNA Induces Inflammatory Responses in Cardiomyocytes and Immune Cells via Toll-like Receptor 7 Signaling. J Biol Chem 290, 26688-26698

Flora GD, Nayak MK (2019): A Brief Review of Cardiovascular Diseases, Associated Risk Factors and Current Treatment Regimes. CPD 25, 4063-4084

Frey N, Olson EN (2003): Cardiac Hypertrophy: The Good, the Bad, and the Ugly. Annu Rev Physiol $\underline{65}$, 45-79

Fu Y, Dominissini D, Rechavi G, He C (2014): Gene expression regulation mediated through reversible $\mathrm{m}^{6} \mathrm{~A}$ RNA methylation. Nat Rev Genet $\underline{15}$, 293-306 
Gao X, Shin Y-H, Li M, Wang F, Tong Q, Zhang P (2010): The Fat Mass and Obesity Associated Gene FTO Functions in the Brain to Regulate Postnatal Growth in Mice. PLoS ONE $\underline{5}$, e14005

Gerken T, Girard CA, Tung Y-CL, Webby CJ, Saudek V, Hewitson KS, Yeo GSH, McDonough MA, Cunliffe S, McNeill LA, et al. (2007): The Obesity-Associated FTO Gene Encodes a 2-Oxoglutarate-Dependent Nucleic Acid Demethylase. Science 318, 1469-1472

Geula S, Moshitch-Moshkovitz S, Dominissini D, Mansour AA, Kol N, Salmon-Divon M, Hershkovitz V, Peer E, Mor N, Manor YS, et al. (2015): ${ }^{6}$ A mRNA methylation facilitates resolution of naïve pluripotency toward differentiation. Science $\underline{347}, 1002-$ 1006

Gilsbach R, Preissl S, Grüning BA, Schnick T, Burger L, Benes V, Würch A, Bönisch U, Günther S, Backofen R, et al. (2014): Dynamic DNA methylation orchestrates cardiomyocyte development, maturation and disease. Nat Commun $\underline{5}, 5288$

Gilsbach R, Schwaderer M, PreissI S, Grüning BA, Kranzhöfer D, Schneider P, Nührenberg TG, Mulero-Navarro S, Weichenhan D, Braun C, et al. (2018): Distinct epigenetic programs regulate cardiac myocyte development and disease in the human heart in vivo. Nat Commun 9, 391

Giudice J, Xia Z, Wang ET, Scavuzzo MA, Ward AJ, Kalsotra A, Wang W, Wehrens XHT, Burge CB, Li W, Cooper TA (2014): Alternative splicing regulates vesicular trafficking genes in cardiomyocytes during postnatal heart development. Nat Commun $\underline{5}, 3603$

Gray M (1998): Angiotensin II stimulates cardiac myocyte hypertrophy via paracrine release of TGF- $\beta 1$ and endothelin-1 from fibroblasts. Cardiovascular Research 40, 352-363

Gustavsson J, Mehlig K, Leander K, Lissner L, Björck L, Rosengren A, Nyberg F (2014): FTO Genotype, Physical Activity, and Coronary Heart Disease Risk in Swedish Men and Women. Circ Cardiovasc Genet $\underline{7}, 171-177$

Haldar SM, McKinsey TA (2014): BET-ting on chromatin-based therapeutics for heart failure. Journal of Molecular and Cellular Cardiology $\underline{74}, 98-102$

Han M, Liu Z, Xu Y, Liu X, Wang D, Li F, Wang Y, Bi J (2020): Abnormality of m6A mRNA Methylation Is Involved in Alzheimer's Disease. Front Neurosci 14, 98

Hartmann AM, Nayler O, Schwaiger FW, Obermeier A, Stamm S (1999): The Interaction and Colocalization of Sam68 with the Splicing-associated Factor YT521-B in Nuclear Dots Is Regulated by the Src Family Kinase p59 fyn. MBoC 10, 3909-3926

Hess ME, Hess S, Meyer KD, Verhagen LAW, Koch L, Brönneke HS, Dietrich MO, Jordan SD, Saletore Y, Elemento O, et al. (2013): The fat mass and obesity associated gene (Fto) regulates activity of the dopaminergic midbrain circuitry. Nat Neurosci $\underline{16}, 1042-1048$

Hill JA, Olson EN (2008): Cardiac Plasticity. New England Journal of Medicine $\underline{358}, 1370$ 1380

Hilleren PJ, Parker R (2003): Cytoplasmic Degradation of Splice-Defective Pre-mRNAs and Intermediates. Molecular Cell $\underline{12}, 1453-1465$

House A, Fatica E, Shah R, Stergar J, Pearce R, Sandlers Y (2020): A protocol for metabolic characterization of human induced pluripotent stem cell-derived cardiomyocytes (iPSCM). MethodsX $\underline{7}, 100572$ 
Hsu PJ, Zhu Y, Ma H, Guo Y, Shi X, Liu Y, Qi M, Lu Z, Shi H, Wang J, et al. (2017): Ythdc2 is an N6-methyladenosine binding protein that regulates mammalian spermatogenesis. Cell Res 27, 1115-1127

Huang $H$, Weng $H$, Sun W, Qin X, Shi $H$, Wu H, Zhao BS, Mesquita A, Liu C, Yuan CL, et al. (2018): Recognition of RNA N6-methyladenosine by IGF2BP proteins enhances mRNA stability and translation. Nat Cell Biol 20, 285-295

Huang H, Weng H, Zhou K, Wu T, Zhao BS, Sun Mingli, Chen Z, Deng X, Xiao G, Auer F, et al. (2019): Histone $\mathrm{H} 3$ trimethylation at lysine 36 guides m6A RNA modification cotranscriptionally. Nature $\underline{567}, 414-419$

Imai Y, Matsuo N, Ogawa S, Tohyama M, Takagi T (1998): Cloning of a gene, YT521, for a novel RNA splicing-related protein induced by hypoxia/reoxygenation. Molecular Brain Research $\underline{53}, 33-40$

Inamdar Arati, Inamdar Ajinkya (2016): Heart Failure: Diagnosis, Management and Utilization. $\operatorname{JCM} \underline{5}, 62$

Institute of Laboratory Animal Resources, National Research Council (eds.): Guide for the care and use of laboratory animals. 6. print; National Acad. Press, Washington 1996

Jackson RJ, Hellen CUT, Pestova TV (2010): The mechanism of eukaryotic translation initiation and principles of its regulation. Nat Rev Mol Cell Biol 11, 113-127

Jaenisch R, Young R (2008): Stem Cells, the Molecular Circuitry of Pluripotency and Nuclear Reprogramming. Cell $132,567-582$

Jain D, Puno MR, Meydan C, Lailler N, Mason CE, Lima CD, Anderson KV, Keeney S (2018): ketu mutant mice uncover an essential meiotic function for the ancient RNA helicase YTHDC2. eLife $\underline{7}$, e30919

Jia G, Fu Y, Zhao X, Dai Q, Zheng G, Yang Y, Yi C, Lindahl T, Pan T, Yang Y-G, He C (2011): N6-Methyladenosine in nuclear RNA is a major substrate of the obesity-associated FTO. Nat Chem Biol 포 885-887

Jonas S, Izaurralde E (2015): Towards a molecular understanding of microRNA-mediated gene silencing. Nat Rev Genet $\underline{16}, 421-433$

Karikó K, Muramatsu H, Welsh FA, Ludwig J, Kato H, Akira S, Weissman D (2008): Incorporation of Pseudouridine Into mRNA Yields Superior Nonimmunogenic Vector With Increased Translational Capacity and Biological Stability. Molecular Therapy $\underline{16}$, $1833-1840$

Ke S, Alemu EA, Mertens C, Gantman EC, Fak JJ, Mele A, Haripal B, Zucker-Scharff I, Moore MJ, Park CY, et al. (2015): A majority of $\mathrm{m}^{6} \mathrm{~A}$ residues are in the last exons, allowing the potential for 3' UTR regulation. Genes Dev 29, 2037-2053

Ke S, Pandya-Jones A, Saito Y, Fak JJ, Vågbø CB, Geula S, Hanna JH, Black DL, Darnell JE, Darnell RB (2017): m6A mRNA modifications are deposited in nascent pre-mRNA and are not required for splicing but do specify cytoplasmic turnover. Genes Dev $\underline{31}, 990$ 1006

Keller A, Dziedzicka D, Zambelli F, Markouli C, Sermon K, Spits C, Geens M (2018): Genetic and epigenetic factors which modulate differentiation propensity in human pluripotent stem cells. Human Reproduction Update 24, 162-175 
Kessler-Icekson G, Barhum Y, Schaper J, Schaper W, Kaganovsky E, Brand T (2002): ANP expression in the hypertensive heart. Exp Clin Cardiol $\underline{7}, 80-84$

Kmietczyk V, Riechert E, Kalinski L, Boileau E, Malovrh E, Malone B, Gorska A, Hofmann C, Varma $E$, Jürgensen $L$, et al. (2019): $m^{6} A-m R N A$ methylation regulates cardiac gene expression and cellular growth. Life Sci Alliance 2, e201800233

Koski GK, Karikó K, Xu S, Weissman D, Cohen PA, Czerniecki BJ (2004): Cutting Edge: Innate Immune System Discriminates between RNA Containing Bacterial versus Eukaryotic Structural Features That Prime for High-Level IL-12 Secretion by Dendritic Cells. J Immunol 172, 3989-3993

Kranz A, Fu J, Duerschke K, Weidlich S, Naumann R, Stewart AF, Anastassiadis K (2010): An improved Flp deleter mouse in C57BI/6 based on Flpo recombinase. Genesis $\underline{48}, 512$ 520

Kumari B, Kumar R, Kumar M (2015): Low complexity and disordered regions of proteins have different structural and amino acid preferences. Mol BioSyst 11, 585-594

Lan T, Li H, Zhang D, Xu L, Liu H, Hao X, Yan X, Liao H, Chen X, Xie K, et al. (2019): KIAA1429 contributes to liver cancer progression through N6-methyladenosine-dependent posttranscriptional modification of GATA3. Mol Cancer $\underline{18}, 186$

Langdon EM, Gladfelter AS (2018): A New Lens for RNA Localization: Liquid-Liquid Phase Separation. Annu Rev Microbiol $\underline{72}, 255-271$

Lee Y, Choe J, Park OH, Kim YK (2020): Molecular Mechanisms Driving mRNA Degradation by m6A Modification. Trends in Genetics $\underline{36}, 177-188$

Lewis YE, Moskovitz A, Mutlak M, Heineke J, Caspi LH, Kehat I (2018): Localization of transcripts, translation, and degradation for spatiotemporal sarcomere maintenance. Journal of Molecular and Cellular Cardiology $\underline{116}, 16-28$

Li A, Chen Y-S, Ping X-L, Yang X, Xiao W, Yang Y, Sun H-Y, Zhu Q, Baidya P, Wang X, et al. (2017): Cytoplasmic m6A reader YTHDF3 promotes mRNA translation. Cell Res $\underline{27}$, 444-447

Li Z, Weng H, Su R, Weng X, Zuo Z, Li C, Huang Huilin, Nachtergaele S, Dong L, Hu C, et al. (2017): FTO Plays an Oncogenic Role in Acute Myeloid Leukemia as a N 6 Methyladenosine RNA Demethylase. Cancer Cell 31, 127-141

Lian X, Zhang J, Azarin SM, Zhu K, Hazeltine LB, Bao X, Hsiao C, Kamp TJ, Palecek SP (2013): Directed cardiomyocyte differentiation from human pluripotent stem cells by modulating Wnt/ $\beta$-catenin signaling under fully defined conditions. Nat Protoc $\underline{8}, 162-$ 175

Liao Y, Castello A, Fischer B, Leicht S, Föehr S, Frese CK, Ragan C, Kurscheid S, Pagler E, Yang $\mathrm{H}$, et al. (2016): The Cardiomyocyte RNA-Binding Proteome: Links to Intermediary Metabolism and Heart Disease. Cell Reports 16, 1456-1469

Linder B, Grozhik AV, Olarerin-George AO, Meydan C, Mason CE, Jaffrey SR (2015): Singlenucleotide-resolution mapping of $\mathrm{m} 6 \mathrm{~A}$ and $\mathrm{m} 6 \mathrm{Am}$ throughout the transcriptome. Nat Methods $\underline{12}, 767-772$

Liu L, Wang Y, Wu J, Liu J, Qin Z, Fan H (2020): N6-Methyladenosine: A Potential Breakthrough for Human Cancer. Molecular Therapy - Nucleic Acids $\underline{19}$, 804-813 
Liu N, Dai Q, Zheng G, He C, Parisien M, Pan T (2015): N(6)-methyladenosine-dependent RNA structural switches regulate RNA-protein interactions. Nature $\underline{518}, 560-564$

Liu N, Zhou KI, Parisien M, Dai Q, Diatchenko L, Pan T (2017): N6-methyladenosine alters RNA structure to regulate binding of a low-complexity protein. Nucleic Acids Res $\underline{45}$, 6051-6063

Liu Q, Chen Y, Auger-Messier M, Molkentin JD (2012): Interaction Between NFkB and NFAT Coordinates Cardiac Hypertrophy and Pathological Remodeling. Circ Res $\underline{110}$, 10771086

Livak KJ, Schmittgen TD (2001): Analysis of Relative Gene Expression Data Using Real-Time Quantitative PCR and the 2- $\triangle \triangle C T$ Method. Methods 25, 402-408

Lodish HF (ed.): Molecular cell biology. 4th ed; W.H. Freeman, New York 2000

Ma H, Wang X, Cai J, Dai Q, Natchiar SK, Lv R, Chen K, Lu Z, Chen H, Shi YG, et al. (2019): N6-Methyladenosine methyltransferase ZCCHC4 mediates ribosomal RNA methylation. Nat Chem Biol $\underline{15}$, 88-94

Malik N, Rao MS: A Review of the Methods for Human iPSC Derivation. In: Lakshmipathy U, Vemuri MC (Eds.): Pluripotent Stem Cells (Methods in Molecular Biology). volume 997; Humana Press, Totowa, NJ 2013, 23-33

Mathiyalagan P, Adamiak M, Mayourian J, Sassi Y, Liang Y, Agarwal N, Jha D, Zhang S, Kohlbrenner E, Chepurko E, et al. (2019): FTO-Dependent $N^{6}$-Methyladenosine Regulates Cardiac Function During Remodeling and Repair. Circulation 139, 518-532

Mauer J, Luo X, Blanjoie A, Jiao X, Grozhik AV, Patil DP, Linder B, Pickering BF, Vasseur J$\mathrm{J}$, Chen $\mathrm{Q}$, et al. (2017): Reversible methylation of m6Am in the $5^{\prime}$ cap controls mRNA stability. Nature $\underline{541}, 371-375$

Mauer J, Sindelar M, Despic V, Guez T, Hawley BR, Vasseur J-J, Rentmeister A, Gross SS, Pellizzoni L, Debart F, et al. (2019): FTO controls reversible m6Am RNA methylation during snRNA biogenesis. Nat Chem Biol $\underline{15}, 340-347$

McKinsey TA, Olson EN (2005): Toward transcriptional therapies for the failing heart: chemical screens to modulate genes. J Clin Invest $\underline{115}, 538-546$

Merino D, Gil A, Gómez J, Ruiz L, Llano M, García R, Hurlé MA, Nistal JF (2018): Experimental modelling of cardiac pressure overload hypertrophy: Modified technique for precise, reproducible, safe and easy aortic arch banding-debanding in mice. Scientific Reports $\underline{8}$

Merkurjev D, Hong W-T, lida K, Oomoto I, Goldie BJ, Yamaguti H, Ohara T, Kawaguchi S, Hirano T, Martin KC, et al. (2018): Synaptic N6-methyladenosine (m6A) epitranscriptome reveals functional partitioning of localized transcripts. Nature Neuroscience 21, 1004-1014

Meyer KD, Jaffrey SR (2017): Rethinking $\mathrm{m}^{6}$ A Readers, Writers, and Erasers. Annu Rev Cell Dev Biol $\underline{33}$, 319-342

Meyer KD, Saletore Y, Zumbo P, Elemento O, Mason CE, Jaffrey SR (2012): Comprehensive analysis of mRNA methylation reveals enrichment in 3' UTRs and near stop codons. Cell 149, 1635-1646 
Meyer KD, Patil DP, Zhou J, Zinoviev A, Skabkin MA, Elemento O, Pestova TV, Qian S-B, Jaffrey SR (2015): 5' UTR m(6)A Promotes Cap-Independent Translation. Cell 163, 999-1010

Michael Love SA: DESeq2. Bioconductor 2017

Moreau P, d'Uscio LV, Shaw S, Takase H, Barton M, Lüscher TF (1997): Angiotensin II Increases Tissue Endothelin and Induces Vascular Hypertrophy: Reversal by ET A Receptor Antagonist. Circulation 96, 1593-1597

Nag AC (1980): Study of non-muscle cells of the adult mammalian heart: a fine structural analysis and distribution. Cytobios $\underline{28}, 41-61$

Nagy A (2000): Cre recombinase: the universal reagent for genome tailoring. Genesis $\underline{26}$, 99 109

Nakamura M, Sadoshima J (2018): Mechanisms of physiological and pathological cardiac hypertrophy. Nature Reviews Cardiology $\underline{15}$, 387-407

Newton AC, Antal CE, Steinberg SF (2016): Protein kinase C mechanisms that contribute to cardiac remodelling. Clinical Science $\underline{130}, 1499-1510$

Nomura S, Satoh M, Fujita T, Higo T, Sumida T, Ko T, Yamaguchi T, Tobita T, Naito AT, Ito $M$, et al. (2018): Cardiomyocyte gene programs encoding morphological and functional signatures in cardiac hypertrophy and failure. Nat Commun $\underline{9}, 4435$

Norton GR, Woodiwiss AJ, Gaasch WH, Mela T, Chung ES, Aurigemma GP, Meyer TE (2002): Heart failure in pressure overload hypertrophy. Journal of the American College of Cardiology $\underline{39}, 664-671$

Patil DP, Chen C-K, Pickering BF, Chow A, Jackson C, Guttman M, Jaffrey SR (2016): m6A RNA methylation promotes XIST-mediated transcriptional repression. Nature $\underline{537}$, 369-373

Patil DP, Pickering BF, Jaffrey SR (2018): Reading m6A in the Transcriptome: m6A-Binding Proteins. Trends in Cell Biology 28, 113-127

Peng S, Xiao W, Ju D, Sun B, Hou N, Liu Q, Wang Yanli, Zhao H, Gao C, Zhang S, et al. (2019): Identification of entacapone as a chemical inhibitor of FTO mediating metabolic regulation through FOXO1. Sci TransI Med 11, eaau7116

Perez-Perri JI, Rogell B, Schwarzl T, Stein F, Zhou Y, Rettel M, Brosig A, Hentze MW (2018): Discovery of RNA-binding proteins and characterization of their dynamic responses by enhanced RNA interactome capture. Nature Communications $\underline{9}$

Perry RP, Kelley DE, Friderici K, Rottman F (1975): The methylated constituents of L cell messenger RNA: Evidence for an unusual cluster at the $5^{\prime}$ terminus. Cell 4 , 387-394

Ping X-L, Sun B-F, Wang L, Xiao W, Yang X, Wang W-J, Adhikari S, Shi Y, Lv Y, Chen Y-S, et al. (2014): Mammalian WTAP is a regulatory subunit of the RNA N6methyladenosine methyltransferase. Cell Res $\underline{24}, 177-189$

Pinto AR, llinykh A, Ivey MJ, Kuwabara JT, D'Antoni ML, Debuque R, Chandran A, Wang L, Arora K, Rosenthal NA, Tallquist MD (2016): Revisiting Cardiac Cellular Composition. Circ Res 118, 400-409 
Pistner A, Belmonte S, Coulthard T, Blaxall B (2010): Murine echocardiography and ultrasound imaging. JoVE 2100

Prasher D, Greenway SC, Singh RB (2020): The impact of epigenetics on cardiovascular disease. Biochem Cell Biol $\underline{98}, 12-22$

Protter DSW, Parker R (2016): Principles and Properties of Stress Granules. Trends in Cell Biology 26, 668-679

Rehmani T, Salih M, Tuana B (2019): Cardiac-Specific Cre Induces Age-Dependent Dilated Cardiomyopathy (DCM) in Mice. Molecules 24, 1189

Ribeiro E de A, Pinotsis N, Ghisleni A, Salmazo A, Konarev PV, Kostan J, Sjöblom B, Schreiner C, Polyansky AA, Gkougkoulia EA, et al. (2014): The Structure and Regulation of Human Muscle a-Actinin. Cell 159, 1447-1460

Ries RJ, Zaccara S, Klein P, Olarerin-George A, Namkoong S, Pickering BF, Patil DP, Kwak $\mathrm{H}$, Lee JH, Jaffrey SR (2019): m6A enhances the phase separation potential of mRNA. Nature $\underline{571}, 424-428$

Rockman HA, Ross RS, Harris AN, Knowlton KU, STEINHELPERt ME, FIELDt LJ, Ross J, Chien KR (1991): Segregation of atrial-specific and inducible expression of an atrial natriuretic factor transgene in an in vivo murine model of cardiac hypertrophy. Proc Natl Acad Sci USA 5

Rogers F (2013): Aortic Stenosis: New Thoughts on a Cardiac Disease of Older People. The Journal of the American Osteopathic Association 113, 820-828

Roost C, Lynch SR, Batista PJ, Qu K, Chang HY, Kool ET (2015): Structure and Thermodynamics of $\mathrm{N}^{6}$-Methyladenosine in RNA: A Spring-Loaded Base Modification. J Am Chem Soc 137, 2107-2115

Rose BA, Force T, Wang Y (2010): Mitogen-Activated Protein Kinase Signaling in the Heart: Angels Versus Demons in a Heart-Breaking Tale. Physiological Reviews 90, 15071546

Rottman F, Shatkin AJ, Perry RP (1974): Sequences containing methylated nucleotides at the 5 ' termini of messenger RNAs: Possible implications for processing. Cell $\underline{3}, 197-199$

Roundtree IA, Evans ME, Pan T, He C (2017): Dynamic RNA Modifications in Gene Expression Regulation. Cell $\underline{169}, 1187-1200$

Schwartz S, Mumbach MR, Jovanovic M, Wang T, Maciag K, Bushkin GG, Mertins P, TerOvanesyan D, Habib N, Cacchiarelli D, et al. (2014): Perturbation of m6A writers reveals two distinct classes of mRNA methylation at internal and 5' sites. Cell Rep $\underline{8}$, 284-296

Shah A, Gandhi D, Srivastava S, Shah KJ, Mansukhani R (2017): Heart Failure: A Class Review of Pharmacotherapy. P T $\underline{42}, 464-472$

Shi H, Wang X, Lu Z, Zhao BS, Ma H, Hsu PJ, Liu C, He C (2017): YTHDF3 facilitates translation and decay of N6-methyladenosine-modified RNA. Cell Res $\underline{27}$, 315-328

Shi H, Zhang X, Weng Y-L, Lu Zongyang, Liu Y, Lu Zhike, Li J, Hao P, Zhang Y, Zhang F, et al. (2018): m6A facilitates hippocampus-dependent learning and memory through YTHDF1. Nature $\underline{563}, 249-253$ 
Shi Y, Inoue H, Wu JC, Yamanaka S (2017): Induced pluripotent stem cell technology: a decade of progress. Nat Rev Drug Discov $\underline{16}, 115-130$

Shinozawa T, Imahashi K, Sawada H, Furukawa H, Takami K (2012): Determination of Appropriate Stage of Human-Induced Pluripotent Stem Cell-Derived Cardiomyocytes for Drug Screening and Pharmacological Evaluation In Vitro. J Biomol Screen 17, 1192-1203

Siasos G, Tsigkou V, Kosmopoulos M, Theodosiadis D, Simantiris S, Tagkou NM, Tsimpiktsioglou A, Stampouloglou PK, Oikonomou E, Mourouzis K, et al. (2018): Mitochondria and cardiovascular diseases-from pathophysiology to treatment. Ann Transl Med $\underline{6}, 256-256$

Śledź P, Jinek M (2016): Structural insights into the molecular mechanism of the m6A writer complex. eLife $\underline{5}$, e18434

Slobodin B, Han R, Calderone V, Vrielink JAFO, Loayza-Puch F, Elkon R, Agami R (2017): Transcription Impacts the Efficiency of mRNA Translation via Co-transcriptional N6adenosine Methylation. Cell $\underline{169}$, 326-337.e12

Soler-Botija C, Gálvez-Montón C, Bayés-Genís A (2019): Epigenetic Biomarkers in Cardiovascular Diseases. Front Genet 10, 950

Sommer AG, Rozelle SS, Sullivan S, Mills JA, Park S-M, Smith BW, lyer AM, French DL, Kotton DN, Gadue P, et al. (2012): Generation of Human Induced Pluripotent Stem Cells from Peripheral Blood Using the STEMCCA Lentiviral Vector. JoVE 4327

Streckfuss-Bömeke K, Wolf F, Azizian A, Stauske M, Tiburcy M, Wagner S, Hübscher D, Dressel R, Chen S, Jende J, et al. (2013): Comparative study of human-induced pluripotent stem cells derived from bone marrow cells, hair keratinocytes, and skin fibroblasts. European Heart Journal 34, 2618-2629

Su YR, Chiusa M, Brittain E, Hemnes AR, Absi TS, Lim CC, Di Salvo TG (2015): Right Ventricular Protein Expression Profile in End-Stage Heart Failure. Pulm Circ $\underline{5}$, 481497

Sun L, Fazal FM, Li P, Broughton JP, Lee B, Tang L, Huang W, Kool ET, Chang HY, Zhang QC (2019): RNA structure maps across mammalian cellular compartments. Nat Struct Mol Biol 26, $322-330$

Sweet ME, Cocciolo A, Slavov D, Jones KL, Sweet JR, Graw SL, Reece TB, Ambardekar AV, Bristow MR, Mestroni L, Taylor MRG (2018): Transcriptome analysis of human heart failure reveals dysregulated cell adhesion in dilated cardiomyopathy and activated immune pathways in ischemic heart failure. BMC Genomics $\underline{19}, 812$

Tang Y, Nyengaard JR, Andersen JB, Baandrup U, Gundersen HJG (2009): The application of stereological methods for estimating structural parameters in the human heart. Anat Rec (Hoboken) 292, 1630-1647

Toischer K, Rokita AG, Unsöld B, Zhu W, Kararigas G, Sossalla S, Reuter SP, Becker A, Teucher N, Seidler T, et al. (2010): Differential Cardiac Remodeling in Preload Versus Afterload. Circulation 122, 993-1003

Toischer K, Zhu W, Hünlich M, Mohamed BA, Khadjeh S, Reuter SP, Schäfer K, Ramanujam D, Engelhardt S, Field LJ, Hasenfuss G (2017): Cardiomyocyte proliferation prevents 
failure in pressure overload but not volume overload. Journal of Clinical Investigation $\underline{127}, 4285-4296$

van Berlo JH, Maillet M, Molkentin JD (2013): Signaling effectors underlying pathologic growth and remodeling of the heart. Journal of Clinical Investigation $\underline{123}, 37-45$

van Tran N, Ernst FGM, Hawley BR, Zorbas C, Ulryck N, Hackert P, Bohnsack KE, Bohnsack MT, Jaffrey SR, Graille M, Lafontaine DLJ (2019): The human 18S rRNA m6A methyltransferase METTL5 is stabilized by TRMT112. Nucleic Acids Research 47, 7719-7733

Walsh S, Pontén A, Fleischmann BK, Jovinge S (2010): Cardiomyocyte cell cycle control and growth estimation in vivo--an analysis based on cardiomyocyte nuclei. Cardiovasc Res $\underline{86}, 365-373$

Wang P, Doxtader KA, Nam Y (2016): Structural basis for cooperative function of Mettl3 and Mettl14 methyltransferases. Mol Cell $\underline{63}, 306-317$

Wang X, He C (2014): Dynamic RNA Modifications in Posttranscriptional Regulation. Molecular Cell $\underline{56}, 5-12$

Wang X, Lu Z, Gomez A, Hon GC, Yue Y, Han D, Fu Y, Parisien M, Dai Q, Jia G, et al. (2014): N6-methyladenosine-dependent regulation of messenger RNA stability. Nature $\underline{505}$, $117-120$

Wang X, Zhao BS, Roundtree IA, Lu Z, Han D, Ma H, Weng X, Chen K, Shi H, He C (2015): N(6)-methyladenosine Modulates Messenger RNA Translation Efficiency. Cell 161, 1388-1399

Wang X, Feng J, Xue Y, Guan Z, Zhang D, Liu Z, Gong Z, Wang Q, Huang J, Tang C, et al. (2016): Structural basis of N6-adenosine methylation by the METTL3-METTL14 complex. Nature $\underline{534}, 575-578$

Warda AS, Kretschmer J, Hackert P, Lenz C, Urlaub H, Höbartner C, Sloan KE, Bohnsack MT (2017): Human METTL16 is a $N^{6}$-methyladenosine $\left(\mathrm{m}^{6} \mathrm{~A}\right)$ methyltransferase that targets pre-mRNAs and various non-coding RNAs. EMBO Rep 므, 2004-2014

Wei C-M, Moss B (1977): Nucleotide sequences at the $N^{6}$-methyladenosine sites of HeLa cell messenger ribonucleic acid. Biochemistry $\underline{16}, 1672-1676$

Widagdo J, Anggono V (2018): The m6A-epitranscriptomic signature in neurobiology: from neurodevelopment to brain plasticity. J Neurochem 147, 137-152

Wilson AJ, Schoenauer R, Ehler E, Agarkova I, Bennett PM (2014): Cardiomyocyte growth and sarcomerogenesis at the intercalated disc. Cell Mol Life Sci $\underline{71}, 165-181$

Wojtas MN, Pandey RR, Mendel M, Homolka D, Sachidanandam R, Pillai RS (2017): Regulation of m6A Transcripts by the $3^{\prime} \rightarrow 5$ ' RNA Helicase YTHDC2 Is Essential for a Successful Meiotic Program in the Mammalian Germline. Molecular Cell $\underline{68}$, 374387.e12

Wu B, Su S, Patil DP, Liu H, Gan J, Jaffrey SR, Ma J (2018): Molecular basis for the specific and multivariant recognitions of RNA substrates by human hnRNP A2/B1. Nat Commun $\underline{9}, 420$ 
Xiao W, Adhikari S, Dahal U, Chen Y-S, Hao Y-J, Sun B-F, Sun H-Y, Li A, Ping X-L, Lai W-Y, et al. (2016): Nuclear m(6)A Reader YTHDC1 Regulates mRNA Splicing. Mol Cell $\underline{61}$, $507-519$

Xu X, Yang D, Ding J-H, Wang W, Chu P-H, Dalton ND, Wang H-Y, Bermingham JR, Ye Z, Liu F, et al. (2005): ASF/SF2-Regulated CaMKIIס Alternative Splicing Temporally Reprograms Excitation-Contraction Coupling in Cardiac Muscle. Cell 120, 59-72

Yang Y, Hsu PJ, Chen Y-S, Yang Y-G (2018): Dynamic transcriptomic m6A decoration: writers, erasers, readers and functions in RNA metabolism. Cell Research $\underline{28}, 616-624$

Yeo GSH, O'Rahilly S (2012): Uncovering the biology of FTO. Mol Metab 1, 32-36

Yu C-C, Ko J-S, Ai T, Tsai W-C, Chen Z, Rubart M, Vatta M, Everett TH, George AL, Chen P$S$ (2016): Arrhythmogenic calmodulin mutations impede activation of smallconductance calcium-activated potassium current. Heart Rhythm $\underline{13}$, 1716-1723

Yu J, Chau KF, Vodyanik MA, Jiang J, Jiang Y (2011): Efficient Feeder-Free Episomal Reprogramming with Small Molecules. PLoS ONE $\underline{6}$, e17557

Yue B, Song C, Yang L, Cui R, Cheng X, Zhang Z, Zhao G (2019): METTL3-mediated N6methyladenosine modification is critical for epithelial-mesenchymal transition and metastasis of gastric cancer. Mol Cancer $\underline{18}, 142$

Zaccara S, Ries RJ, Jaffrey SR (2019): Reading, writing and erasing mRNA methylation. Nature Reviews Molecular Cell Biology 20, 608-624

Zhang C, Fu J, Zhou Y (2019): A Review in Research Progress Concerning m6A Methylation and Immunoregulation. Front Immunol $\underline{10}, 922$

Zhang S, Zhao BS, Zhou A, Lin K, Zheng S, Lu Z, Chen Y, Sulman EP, Xie K, Bögler O, et al. (2017): $m 6$ A Demethylase ALKBH5 Maintains Tumorigenicity of Glioblastoma Stemlike Cells by Sustaining FOXM1 Expression and Cell Proliferation Program. Cancer Cell $\underline{31}, 591-606 . e 6$

Zhang T, Maier LS, Dalton ND, Miyamoto S, Ross J, Bers DM, Brown JH (2003): The $\delta_{c}$ Isoform of CaMKII Is Activated in Cardiac Hypertrophy and Induces Dilated Cardiomyopathy and Heart Failure. Circulation Research 92, 912-919

Zhang Y: RNA-induced Silencing Complex (RISC). In: Dubitzky W, Wolkenhauer O, Cho K-H, Yokota H (Eds.): Encyclopedia of Systems Biology. Springer New York, New York, NY 2013, 1876-1876

Zheng G, Dahl JA, Niu Y, Fedorcsak P, Huang C-M, Li CJ, Vågbø CB, Shi Y, Wang W-L, Song $\mathrm{S}-\mathrm{H}$, et al. (2013): ALKBH5 is a mammalian RNA demethylase that impacts RNA metabolism and mouse fertility. Mol Cell $\underline{49}, 18-29$

Zhou KI, Shi H, Lyu R, Wylder AC, Matuszek Ż, Pan JN, He C, Parisien M, Pan T (2019): Regulation of Co-transcriptional Pre-mRNA Splicing by m6A through the LowComplexity Protein hnRNPG. Molecular Cell $\underline{76}$, 70-81.e9

Zhou P, Pu WT (2016): Recounting Cardiac Cellular Composition. Circ Res 118, 368-370

Zhu T, Roundtree IA, Wang P, Wang X, Wang L, Sun C, Tian Y, Li J, He C, Xu Y (2014): Crystal structure of the YTH domain of YTHDF2 reveals mechanism for recognition of N6-methyladenosine. Cell Res $\underline{24}, 1493-1496$ 
Zlabinger K, Spannbauer A, Traxler D, Gugerell A, Lukovic D, Winkler J, Mester-Tonczar J, Podesser B, Gyöngyösi M (2019): MiR-21, MiR-29a, GATA4, and MEF2c Expression Changes in Endothelin-1 and Angiotensin II Cardiac Hypertrophy Stimulated Isl-1+Sca$1+\mathrm{c}$-kit+ Porcine Cardiac Progenitor Cells In Vitro. Cells $\underline{8}, 1416$

Zou S, Toh JDW, Wong KHQ, Gao Y-G, Hong W, Woon ECY (2016): N6-Methyladenosine: a conformational marker that regulates the substrate specificity of human demethylases FTO and ALKBH5. Sci Rep $\underline{6}, 25677$

Landini G. https://imagej.net/Auto_Threshold\#Li

World Health Organization. https://www.who.int/news-room/fact-sheets/detail/cardiovasculardiseases-(cvds); retrieved 06/16/2020 


\section{Acknowledgements}

This project took a lot of work and effort, none of which would have been possible without the people who helped and supported me during the last years. First of all, I want to thank Karl for the opportunity to work in his group and for all his advises and guidance which gave me the confidence to pursue this work.

I would also like to thank André Fischer and Steven Johnsen for their valued advice and ideas in our Thesis committee meetings. Their input and knowledge helped me a lot during my project. I furthermore want to thank Prof. Dörthe Katschinski, Prof. Wolfram-Hubertus Zimmermann and Prof. Bernd Wollnik who agreed to join my examination board.

I want to thank the whole Toischer-Lab for the nice working atmosphere every day. Sabrina, Joanna, Kirsten, Alessya, thank you for your time, effort and assistance with my labwork. Belal, thanks for your ideas and funny conversations. Nelly and Alessa, thank you very much for your great work and motivation. Your invaluable input to this project rounded everything up.

I especially want to thank Tea Berulava for being my collaboration partner. Thank you, Tea, for all your time, analysis and explanations whenever I struggled! Without you, this project would not have been possible! Also, many thanks to Katherine Bohnsack and Markus Bohnsack for making polysomal analysis possible.

Dawid, thanks for being an awesome Labpartner, for all your advice and scientific input. Especially for all your time proofreading this thesis. I enjoyed every day in the Lab, may the force be with you, always!

I want to express my gratitude to the SFB1002 service unit Marcel, Roland and Sarah and to the animal caretakers. Thank you, Tobias, Ilka, Nina and Romina for taking care of my mice. It was good to know they were taken care of as good as possible.

I also give many thanks to Katrin Streckfuß-Bömecke and her group, for teaching me iPSC cell culture and supplying me with cells whenever needed.

Special thanks go to Cera. Thank you for making this thesis also 'enjoyable' to native speakers and for all the time you invested for me! Thanks to Andreas, Jerome, Daniel, Lisa and Irina, for countless coffees, lunches and great fun.

Last but not least I want to thank my parents and grandparents. Thank you for your support and giving me the opportunity to follow my passion for science and making my studies possible. Nina, thank you for all your patience and cheering me up. You got me through everything. I am looking forward for our future to come. 


\section{Curriculum Vitae}

Name

Date of birth

Place of birth
Eric Buchholz

18th of September 1991

Bergisch Gladbach, Germany

\section{Education}

Since $\quad$ 1/2017 University Medical Center Göttingen, Göttingen, Germany

Molecular Medicine Graduate Programme, PhD-Project

10/2014-10/2016 University Duisburg-Essen, Campus Essen, Germany

Degree: M.Sc. Master of Science "Medizinische Biologie (Medical Biology)"

9/2011 - 7/2014 Hochschule Bonn-Rhein-Sieg, University of Applied Sciences, Rheinbach, Germany

Degree: B.Sc. Bachelor of Science "Applied Biology"

8/2002 - 7/2011 Gymnasium der Stadt Lennestadt, Lennestadt-Altenhundem, Germany

Degree: Abitur (High school diploma)

\section{Practical Experience}

Since 1/2017 PhD-Project (SFB1002): 'RNA methylation in Cardiac Hypertrophy and Heart Failure'

3/2016-8/2016 West German Cancer Center, University Hospital Essen, Germany

Research group 'Vascular Oncology and Metastasis' - Master Project

'Unravel Bisphenol dynamics on Ras- dependent signaling in Melanoma'

1/2016-2/2016 West German Cancer Center, University Hospital Essen, Germany

Research group 'Molecular Oncology' - Internship

'Retroviral transfection and transduction of lung cancer cells and isolation of single cell clones'

11/2015-1/2016 West German Cancer Center, University Hospital Essen, Germany

Research group 'Vascular Oncology and Metastasis' - Internship

'Attempt to determine the IC50 concentration of Bisphenol-A by using the MTTassay' 
10/2015-11/2015 University Duisburg-Essen, Campus Essen, Germany

Research group 'Bionanophysics' - Internship

'Determination of DNA-origami concentrations by quantitative Real-Time Polymerase Chain Reaction ( $q R T-P C R)$ '

1/2014-5/2014 Institute for Cancer Research at the Norwegian University of Science and Technology (NTNU), Trondheim, Norway - Bachelor Project

'Validating the predicted effect of kinase-inhibitors against a gastric cancer cell line by Western Blotting'

Scientific Achievements

Publications

- Changes in m6A RNA methylation contribute to heart failure progression by modulating translation (Eur J Heart Fail. 2020 Jan;22(1):54-66. doi: 10.1002/ejhf.1672. Epub 2019 Dec 17.)

Berulava $T^{*}$ \& Buchholz E* Ellerdashvilli $V$, Pena T, Islam R, Lbik D, Mohamed BA, Renner A, von Lewinski D, Sacherer M, Sloan KE, Bohnsack MT, Jain G, Capece V, Cleve N, Burkhardt S, Hasenfuss $G$, Fischer A, Toischer K

*shared co-first authorship

- Assessing the role of extracellular signal-regulated kinases 1 and 2 in volume overloadinduced cardiac remodelling. (ESC Heart Fail. 2019 Oct;6(5):1015-1026. doi: 10.1002/ehf2.12497. Epub 2019 Jul 19.)

Jochmann S, Elkenani M, Mohamed BA, Buchholz E, Lbik D, Binder L, Lorenz K, Shah AM, Hasenfuß G, Toischer K, Schnelle $M$.

- Genetic deletion of calcium/calmodulin-dependent protein kinase type II delta does not mitigate adverse myocardial remodeling in volume-overloaded hearts. (Sci Rep. $2019 \mathrm{Jul}$ 8;9(1):9889. doi: 10.1038/s41598-019-46332-3.)

Mohamed BA, Elkenani M, Jakubiczka-Smorag J, Buchholz E, Koszewa S, Lbik D, Schnelle M, Hasenfuss $G$, Toischer $K$.

Participation in Scientific Meetings

- European Society of Cardiology Congress 2018 - Munich (Poster and Scientific Talk)

- European Society of Cardiology Congress 2019 - Paris (Poster)

- Heart \& Brain Symposium 2019 - Göttingen (Poster)

- $85^{\text {th }}$ Annual Meeting of the German Society of Cardiology (DGK) 2019- Mannheim (Poster)

- Gordon Research Conference "Epigenetic Regulation of Cardiovascular Disease"- Hong Kong (Poster) 
$\underline{\text { Teaching Activities }}$

2018

Medical Dissertation supervision Nelly Wery von Limont

2019

Medical Dissertation supervision Alessa Pommeranz

2018

Lab rotation Tabea Quilitz

International Experience

8/2013 - 6/2014 Høgskolen I Sør-Trøndelag (HiST), Trondheim, Norway

Erasmus student in the B. Sc. program 'Bioingeniør (Bioengineering)'

$6 / 2014$

European Cruise Service, Trondheim, Norway

- Work as tourist guide for German and British or American tourists

- Guided tours in German and English language through the city Trondheim

Other work experience

3/2015 - 11/2016 Öko-Konzept GmbH, Velbert, Germany

- Representative on trade fairs

- Communication with interested customers

- Providing information about the company

Voluntary work

Molecular Medicine Graduate Program (6/2018 - 6/2019)

- Student Representative

LMB-GGNB Graduate Life Sciences Symposium 2019 (7/2019)

- Organization Committee 


\section{Co-Author release form}

For the publication entitled "Changes in m6A RNA methylation contribute to heart failure progression by modulating translation".

Eric Buchholz has my permission to include material, which was accepted for publication, of which I was a co-author, in his doctoral dissertation.

Tea Berulava*, Eric Buchholz*, Vakhtang Elerdashvili, Tonatiuh Pena, Md Rezaul Islam, Dawid Lbik, Belal A. Mohamed, Andre Renner, Dirk von Lewinski, Michael Sacherer, Katherine E. Bohnsack, Markus T. Bohnsack, Gaurav Jain, Vincenzo Capece, Nicole Cleve, Susanne Burkhardt, Gerd Hasenfuss, Andre Fischer, and Karl Toischer

"Changes in m6A RNA methylation contribute to heart failure progression by modulating translation"

*Authors contributed equally as fist authors

Tea Berulava

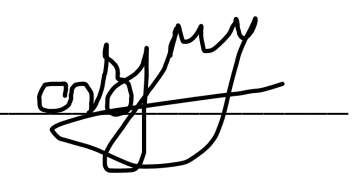




\title{
Changes in m6A RNA methylation contribute to heart failure progression by modulating
} translation

\author{
Tea Berulava ${ }^{1 \dagger}$, Eric Buchholz $2,7 \dagger$, Vakhtang Elerdashvili1,3, Tonatiuh Pena ${ }^{1,3}$, \\ Md Rezaul Islam', Dawid Lbik2,7, Belal A. Mohamed2,7, Andre Renner4, \\ Dirk von Lewinski ${ }^{5}$, Michael Sacherer ${ }^{5}$, Katherine E. Bohnsack ${ }^{6}$, \\ Markus T. Bohnsack ${ }^{6}$, Gaurav Jain ${ }^{1,3}$, Vincenzo Capece ${ }^{1}$, Nicole Cleve', \\ Susanne Burkhardt ${ }^{1}$, Gerd Hasenfuss ${ }^{2,7}$, Andre Fischer ${ }^{1,8,9} *:$, and Karl Toischer $2,7 *:$
}

${ }^{1}$ Department for Epigenetics and Systems Medicine in Neurodegenerative Diseases, German Center for Neurodegenerative Diseases (DZNE), Göttingen, Germany; ${ }^{2}$ Clinic for Cardiology and Pneumology, University Medical Center, Göttingen, Germany; ${ }^{3}$ Bioinformatics Unit, German Center for Neurodegenerative Diseases (DZNE), Göttingen, Germany; ${ }^{4}$ Clinic for Thoracic and Cardiovascular Surgery, Heart and Diabetes Centre NRW, Ruhr-University Bochum, Bochum, Germany; ${ }^{5}$ Department of Cardiology, Medical University Graz, Graz, Austria; ${ }^{6}$ Department of Molecular Biology, University Medical Center, Göttingen, Germany; ${ }^{7}$ German Centre for Cardiovascular Research (DZHK), partner site Göttingen, Göttingen, Germany; ${ }^{8}$ Department of Psychiatry and Psychotherapy, University Medical Center, Göttingen, Germany; and ${ }^{9}$ Cluster of Excellence "Multiscale Bioimaging: from Molecular Machines to Networks of Excitable Cells" (MBExC), University of Göttingen, Germany

Received 28 June 2019; revised 15 September 2019; accepted 15 October 2019; online publish-ahead-of-print 17 December 2019

Aims

Deregulation of epigenetic processes and aberrant gene expression are important mechanisms in heart failure. Here we studied the potential relevance of m6A RNA methylation in heart failure development.

Methods

and results

We analysed m6A RNA methylation via next-generation sequencing. We found that approximately one quarter of the transcripts in the healthy mouse and human heart exhibit m6A RNA methylation. During progression to heart failure we observed that changes in m6A RNA methylation exceed changes in gene expression both in mouse and human. RNAs with altered m6A RNA methylation were mainly linked to metabolic and regulatory pathways, while changes in RNA expression level mainly represented changes in structural plasticity. Mechanistically, we could link m6A RNA methylation to altered RNA translation and protein production. Interestingly, differentially methylated but not differentially expressed RNAs showed differential polysomal occupancy, indicating transcription-independent modulation of translation. Furthermore, mice with a cardiomyocyte restricted knockout of the RNA demethylase Fto exhibited an impaired cardiac function compared to control mice.

Conclusions We could show that $\mathrm{m} 6 \mathrm{~A}$ landscape is altered in heart hypertrophy and heart failure. $\mathrm{m} 6 \mathrm{~A}$ RNA methylation changes lead to changes in protein abundance, unconnected to mRNA levels. This uncovers a new transcription-independent mechanisms of translation regulation. Therefore, our data suggest that modulation of epitranscriptomic processes such as m6A methylation might be an interesting target for therapeutic interventions.

Keywords Heart failure • RNA methylation • Epitranscriptomics • Translation

*Corresponding authors. Karl Toischer, Department of Cardiology and Pneumology, Georg-August-University, Universität Göttingen, Robert-Koch-Str. 40,37075 Göttingen, Germany. Tel: +49551 3966318, Fax: +49551 3922953, Email: ktoischer@med.uni-goettingen.de

Andre Fischer, Department for Epigenetics and Systems Medicine, German Center for Neurodegenertive diseases, von Siebold Str. 3A, 37075 Göttingen, Germany Tel: +49 551 3961211, Fax: +49 551 3961214, Email: andre.fischer@dzne.de

These authors contributed equally as first authors.

These authors contributed equally as senior authors.

(c) 2019 The Authors. European Journal of Heart Failure published by John Wiley \& Sons Ltd on behalf of European Society of Cardiology. This is an open access article under the terms of the Creative Commons Attribution-NonCommercial License, which permits use, distribution and reproduction in any medium, provided the original work is properly cited and is not used for commercial purposes. 


\section{Introduction}

Heart failure, characterized by reduced cardiac function and left ventricular dilatation, is a leading cause of hospital admission and mortality. ${ }^{1}$ This process is accompanied by increased apoptosis, fibrosis and changes in gene expression. ${ }^{2,3}$ Until now, inhibition of neuroendocrine stimulation is the only treatment for heart failure; however, the therapeutic efficacy of this approach is limited and cannot prevent the eventual progression of the disease. ${ }^{4}$ Therefore, additional therapeutic options are needed.

There is increasing evidence that aberrant gene expression, orchestrated by transcription factors and epigenetic processes such as non-coding RNAs, DNA and histone modifications, represents a key event in heart failure and could thus offer new ways for therapeutic intervention. ${ }^{5,6}$ While methylation of DNA seems to be important during maturation of the heart, ${ }^{7}$ the role of RNA modifications has not been studied in detail until now.

N6-adenosine methylation (m6A) of RNA transcripts is the most prevalent modification found in many classes of RNA. ${ }^{8}$ Similar to epigenetic changes in DNA and histone modifications, m6A in RNA is dynamic and reversible. ${ }^{8-10}$ In all classes of RNA, m6A mainly occurs within a highly-conserved consensus motif identified as $\mathrm{RRACH}(\mathrm{R}=\mathrm{G}$ or $\mathrm{A}, \mathrm{H}=\mathrm{A}, \mathrm{C}$ or $\mathrm{U})$. The formation of $\mathrm{m} 6 \mathrm{~A}$ is regulated by methyltransferases (METTL3, METTL14, WTAP, METTL16) ${ }^{11,12}$ and demethylases (FTO, ALKBH5). ${ }^{13,14} \mathrm{~m} 6 \mathrm{~A}$ recognizing proteins such as members of the YTH domain protein family and HNRNPA2B1 are involved in processes regulating the fate of target transcripts. The current data suggest that the degree and the pattern of methylation of mRNAs can affect their splicing, transport, storage, translation and/or decay. ${ }^{10}$

At present, there is only one recent study which reported a role for m6A in the peri-infarct zone after myocardial infarction. ${ }^{15}$ In our study, we consequently applied transcriptome-wide approaches and defined changes in $\mathrm{m} 6 \mathrm{~A}$ in mouse and human heart failure development. We found that m6A RNA methylation appears to affect cardiac signalling and metabolic processes by modulation of translation. Our data provide evidence that m6A RNA methylation is involved in heart failure development and might be a novel therapeutic target.

\section{Methods}

See online supplementary Methods S1 for detailed description. The investigation conforms to the principles outlined in the Declaration of Helsinki and the Guide for the Care and Use of Laboratory Animals (NIH publication No. 85-23, revised 1996). All patients provided written informed consent for the use of cardiac tissue samples. Surgery on mice was done using a minimally invasive approach. Echocardiography was performed on anaesthetized animals.

RNA isolation from left ventricles was performed with Trizol reagent according to the manufacturer's instruction. DNase treated and fragmentized RNA was subjected first to immunoprecipitation with anti-m6A antibody (Synaptic Systems, \#202003) and then to high-throughput sequencing on Illumina 2000 platform. Generated reads were mapped to $\mathrm{mm} 10$ and $\mathrm{hg} 19$, and peaks showing significant enrichment for $\mathrm{m6 \textrm {A }}$ in immunoprecipitated samples compared to corresponding input samples were detected with the MeTPeak package. ${ }^{16}$ For the polysomal occupancy experiment, RNA extracted from polysomal fractions of the snap-frozen tissues was sequenced on the Illumina HiSeq2000. Differential methylation analysis was done using an in-house developed pipeline. Deseq2 was used for differential gene expression and differential polysome binding analysis. mRNAs showing significant (Padj < 0.05 ) and at least two-fold change in $\mathrm{m} 6 \mathrm{~A}$ levels, mRNA levels and polysome binding are reported in this study. Differential m6A methylation was further verified using qRT-PCR on RNAs immunoprecipitated with anti-m6A antibody without fragmentation. Western blot analysis for different protein level evaluation was performed following manufacturer's instructions for the corresponding antibodies. We used the ClueGo plug-in in the Cytoscape software for pathway analysis for genes identified in different experiments.

\section{Results}

\section{m6A RNA methylation in the adult heart}

Fto, Mettl3 and Mettl14 as the key regulators of m6A RNA methylation are expressed in the adult mouse heart (Figure $1 A$ and online supplementary Figure S9). Transcriptome-wide analysis of $\mathrm{m} 6 \mathrm{~A}$ RNA methylation via m6A-specific methylated RNA immunoprecipitation followed by next-generation sequencing (MeRIP-Seq) ${ }^{17}$ revealed that $24 \%$ of all detected transcripts carry m6A marks $(n=5)$. More specifically, we detected 3208 peaks linked to 2164 transcripts (Figure 1B and online supplementary Table S4). The quality of our data was confirmed by an unbiased motif search using the detected m6A peaks as input that identified the previously reported $\mathrm{m} 6 \mathrm{~A}$ consensus sequence RRACH (Figure 1C). Relative quantification of $\mathrm{m} 6 \mathrm{~A}$ peaks across all transcripts showed a distribution similar as in other tissues ${ }^{17-19}$ with an enrichment of $\mathrm{m} 6 \mathrm{~A}$ toward the translation end site (Figure 1D).

The majority of the $\mathrm{m} 6 \mathrm{~A}$-containing transcripts carried this mark in the $5^{\prime} U T R$ or $3^{\prime} U T R$ and the coding sequence (CDS). Only a negligible number of transcripts were exclusively m6A-methylated in $5^{\prime}$ UTR $(n=9)$ or $3^{\prime}$ UTR $(n=163)$ (Figure 1E). A gene ontology (GO) analysis revealed that transcripts with $\mathrm{m} 6 \mathrm{~A}$ in the 5'UTR and/or CDS were linked to energy metabolism, mitochondrial function and intra-cellular signalling, while transcripts methylated in the $3^{\prime}$ UTR mostly code for proteins involved in pathways linked to more specific metabolic processes such as 'acetyl-CoA or glycerol biosynthesis' and 'positive regulation of protein dephosphorylation' (Figure $1 F$ ).

Transcripts that carry m6A in the 5'UTR and CDS showed a mild positive correlation of m6A marks with expression level $(r=0.10$, $P=5.86 \mathrm{e}-07$; Figure $1 G$ ), whereas methylation marks within the $3^{\prime} U T R$ showed a mild negative correlation $(r=-0.08, P=0.006$; Figure $1 G$ ). We also detected long non-coding RNAs with m6A marks (online supplementary Figure S1A), but here m6A was not correlated to its expression level $(r=0.06, P=0.72$; online supplementary Figure S1B). A detailed annotation of the m6A-containing non-coding peaks is found in online supplementary Table S4.

\section{Cardiac hypertrophy and failure are linked to substantial changes in m6A}

Next, we studied m6A RNA methylation during the progression of heart failure. Therefore we used the mouse model of 


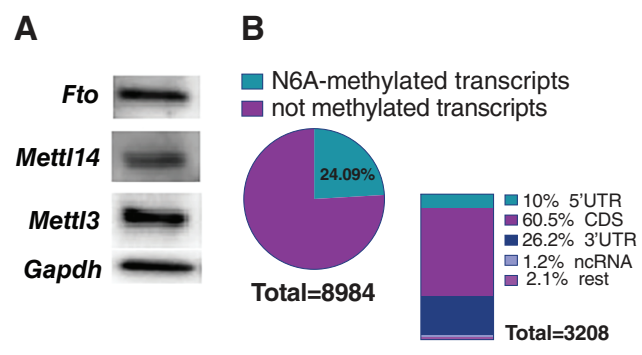

E

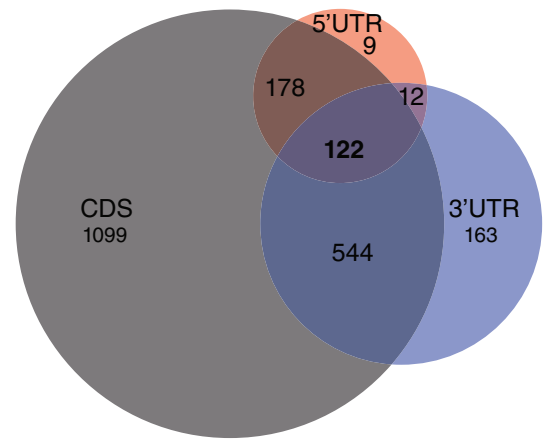

$\mathbf{F}$

5'UTR and CDS

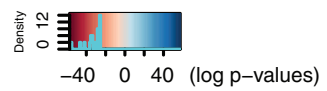

cellular protein metabolic process

mitochondrial electron transport, ubiquinol to cyt. c negative regulation of protein complex assembly

mitochondrial respiratory chain complex III assembly mitochondrion organization

mitochondrial translation

negative regulation of intracellular signal transduction sarcomere organization

regulation of cellular protein catabolic process

muscle tissue development

\section{3'UTR}

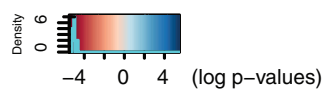

positive regulation of smooth muscle cell proliferation atrioventricular valve morphogenesis

acyl-CoA biosynthetic process

positive regulation of protein dep

positive regulation of protein dephosphorylation

glycerolipid biosynthetic process

pos. regulation of proteasomal ubiquitin-dependent protein catabolic process lysosomal transport
C

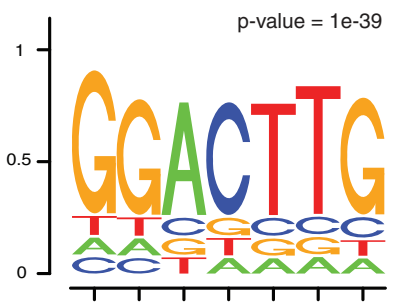

G

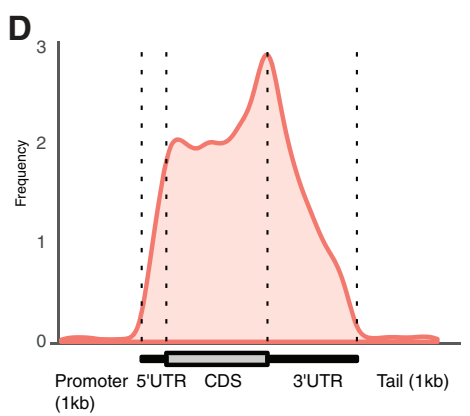

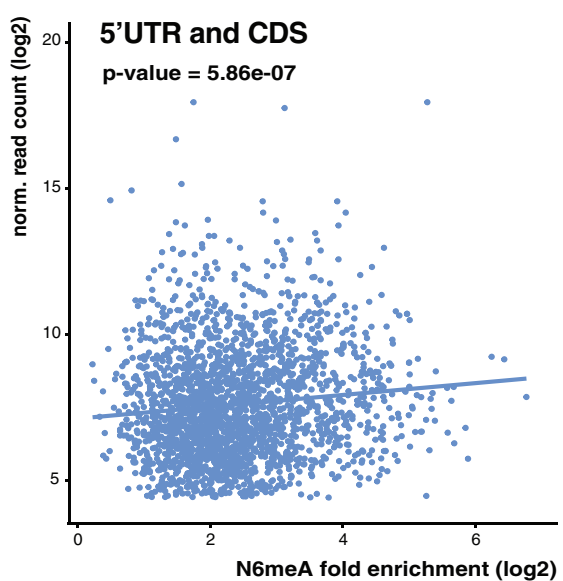

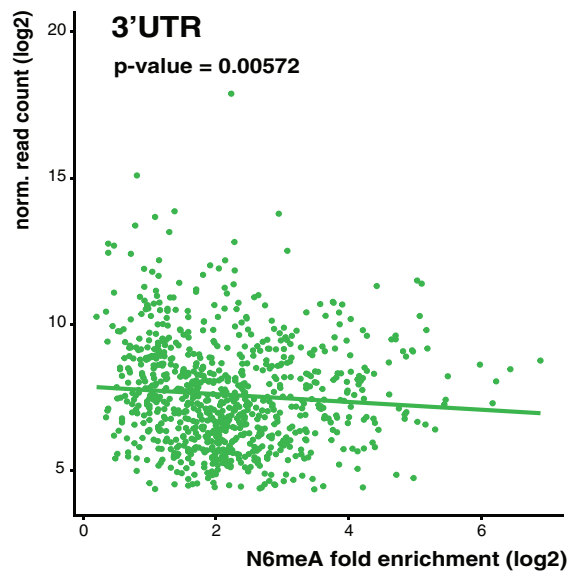

Figure 1 m6A RNA methylation in the healthy mouse heart. (A) Representative western blot images showing expression of Fto, Mettl3 and Mettl14 protein in mouse heart tissue. (B) Pie and bar charts showing the amount and distribution of m6A RNA methylation in the mouse heart. $(C)$ Sequence motif identified within m6A peaks. $(D)$ Distribution of m6A peaks across mRNA transcripts. (E) Venn diagram showing methylation marks across transcript regions. $(F)$ Heat map showing pathway analysis of transcripts that carry m6A marks in either $5^{\prime} U T R$ and CDS or $3^{\prime}$ UTR. (G) Correlation analysis between transcript level and m6A methylation at 5'UTR and CDS (upper panel) and $3^{\prime} \cup T R$ (lower panel). $n=5$ per group.

transverse aortic constriction (TAC) to induce pressure overload. ${ }^{3}$ One week after TAC mice responded with compensated hypertrophy (Figure 2A), whereas after 8 weeks heart failure developed (Figure 2B). In addition to m6A RNA methylation, we also analysed expression changes from the same material by RNA Seq.
We detected a number of differentially expressed genes either 1 or 8 weeks after TAC that were mainly linked to structural cardiac plasticity pathways ( $n=6$ per group; online supplementary Figure S2, Tables S3 and S4). Using the same cut-offs as for the detection of differential methylated transcripts (Log2FC $>1$, Padj $<0.05)$ we observed that at both time points after TAC 
A

heart hypertrophy
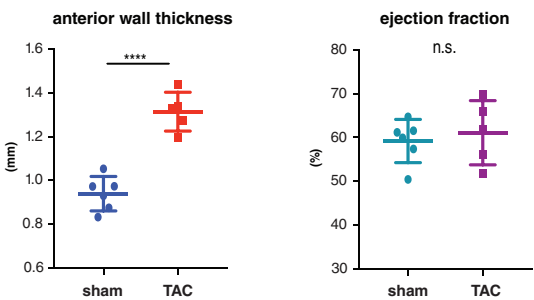

C

heart hypertrophy

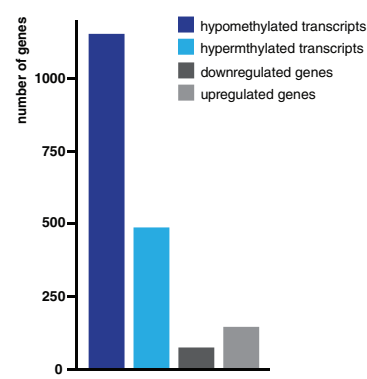

E

heart hypertrophy
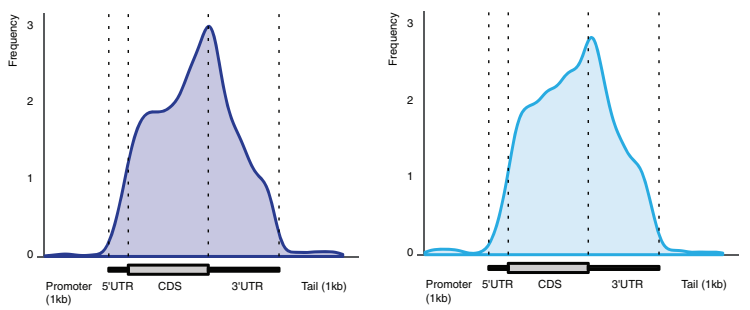

$\square$ hypomethylated transcripts $\quad \square$ hypermethylated transcripts

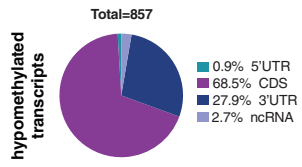

B

heart failure
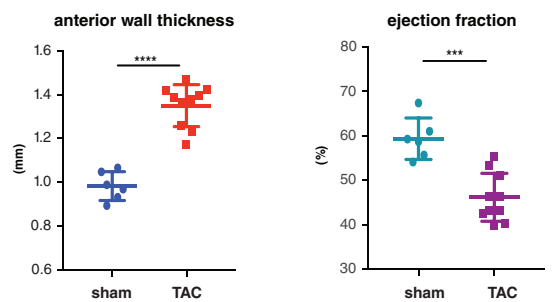

D
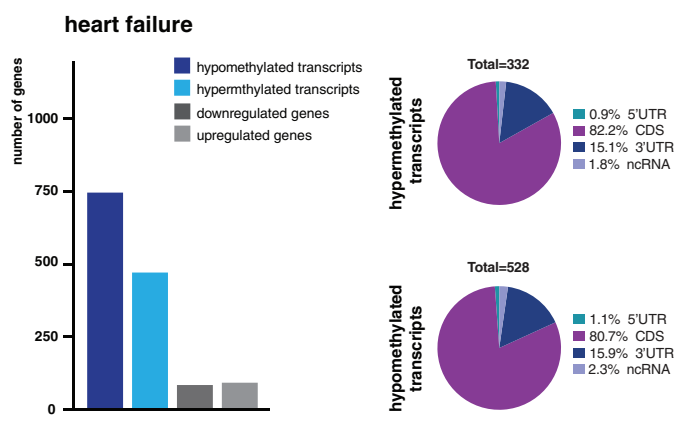

G
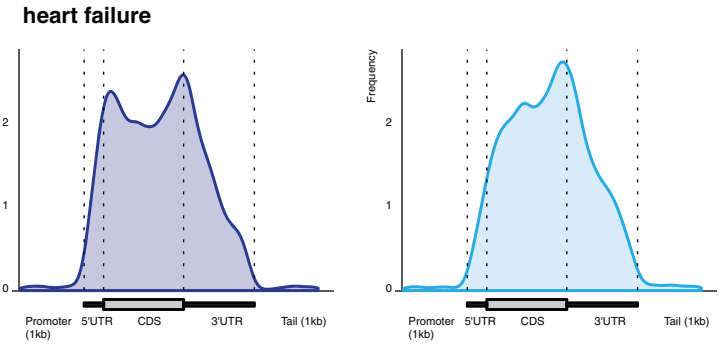

$\mathbf{F}$
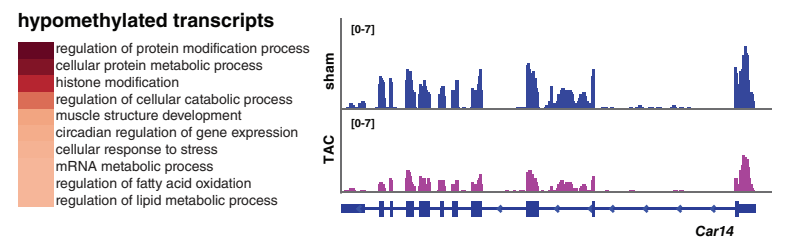

hypermethylated transcripts

negative regulation of ERK1 and ERK2 cascade

negative regulation of transcription from RNA polymerase II promoter

extracellular matrix organization

wound healing

response to oxygen levels

response to hydrogen peroxide

collagen biosynthetic process

actin filament organization

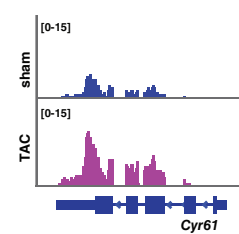

H
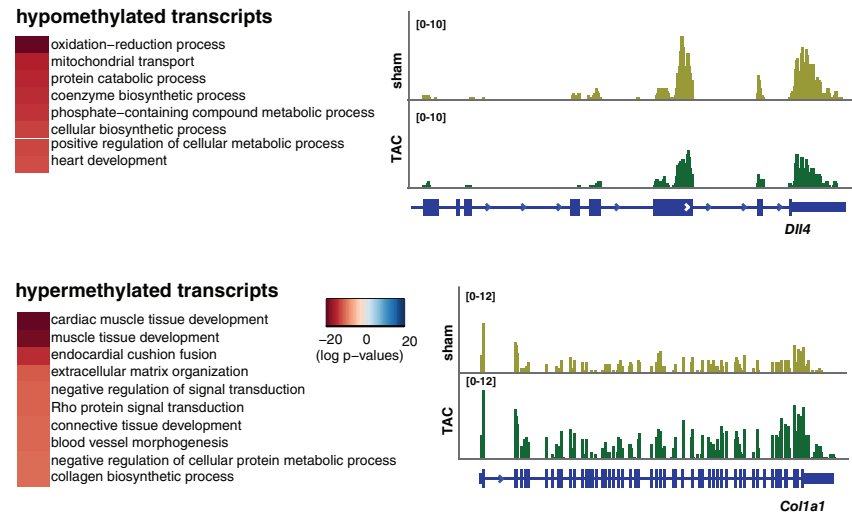

Figure 2 Legend on next page. 
the number of transcripts with significant changes in m6A was much higher than the number of differentially expressed genes [Figure 2C and 2D; 1 week: 1638 transcripts differentially methylated, 217 differentially expressed (online supplementary Figure S2 and Table S3); 8 weeks: 1215 transcripts differentially methylated and 174 differentially expressed (online supplementary Figure S2 and Table S4)]. The global distribution of m6A marks across hyperand hypomethylation 1 week after TAC did not differ from control (Figure $2 E$ and $2 G$ ), whereas in case of 8 weeks after TAC a significant shift toward hypomethylation specifically at the transcription start site (TSS) was visible with an area under the curve (AUC) of 0.2733 (95\% confidence interval $0.2731-0.2735$ ) for hypomethylation whereas AUC for hypermethylation at 5'UTR was 0.1617 (95\% confidence interval 0.1614-0.1619) (Figure 2G). Transcripts affected by m6A RNA methylation were substantially different from the transcripts affected at the level of expression (online supplementary Figure S3, Tables S3 and S4), suggesting that changes in m6A RNA methylation and the regulation of transcript level likely represent distinct cellular processes.

Next, we performed a GO analysis of differentially methylated transcripts. Interestingly, only few pathways involved in contractile or structural processes could be detected. However, both hypoand hypermethylated transcripts in case of heart hypertrophy were enriched in GO categories linked to metabolic processes and gene expression regulation such as 'histone modification' and 'cellular response to stress' (Figure 2F). Additionally, hypermethylated transcripts were linked to signalling pathways such as ERK1/2 signalling (Figure 2F). The differentially methylated transcripts detected in the heart failure model were also linked to metabolic processes and mitochondrial functions and especially hypermethylated transcripts were also involved in cardiac muscle development (Figure $2 \mathrm{H}$ ).

\section{m6A RNA methylation in the human heart}

The key enzymes, METTL3, METTL4 and FTO, were expressed in the human heart (Figure $3 A$ and online supplementary Figure S9). MeRIP-Seq analysis from non-failing hearts ( $n=5$; online supplementary Table S2) showed a substantial number of transcripts carrying m6A marks (Figure 3B and online supplementary Table S5) and a motif search confirmed the previously described m6A RNA methylation motif (Figure 3C).

The distribution of $\mathrm{m} 6 \mathrm{~A}$ along transcripts was similar to the mouse (Figure 3D and online supplementary Figure S5). Also, in line with the mouse data, most m6A marks occurred in the CDS and $5^{\prime}$ UTR of the corresponding transcripts (Figure $3 E$ ). A substantial number of transcripts carried $\mathrm{m} 6 \mathrm{~A}$ marks exclusively within the 3'UTR (Figure 3E). GO term analysis revealed that m6A containing transcripts encoded proteins linked to cardiomyocyte functions such as 'sarcomere organization', intracellular signalling and metabolic pathways (Figure $3 F$ and $3 G$ ). Similar to the mouse data, we observed a mild correlation of transcript levels and m6A RNA methylation at the $5^{\prime} U T R$ and CDS $(r=0.19, P=3.85 \mathrm{e}-08)$, while no significant correlation was seen for the $3^{\prime} U T R(r=-0.05$, $P=0.3248$ ) (Figure $3 H$ ). Comparison of the m6A-containing transcripts detected in the healthy mouse and human heart revealed a significant overlap of m6A-containing transcripts among the two species (representation factor 7.6, $P<2.37$ e-21; Figure 3 I). It has to be mentioned that more m6A methylated transcripts were detected in mouse heart when compared to the human heart (Figures $1 B$ and $3 B$ ). While these data likely reflect species differences, we cannot exclude that also methodological issues play a role. For example, the preparation of tissue takes $5 \mathrm{~min}$ in case of humans but less than $1 \mathrm{~min}$ in the mouse model, which correlates with RNA integrity values [RIN; $9.00 \pm 0.23$ for mouse and $7.80 \pm 0.57$ for human (mean \pm standard deviation)]. Transcripts that were m6A methylated in both mouse and humans mainly code for proteins linked to metabolic processes, and heart and circulation system development (Figure 3I).

Next, we performed MeRIP-Seq and RNA-Seq analysis of non-failing and end-stage heart failure biopsies ( $n=6$ per group; online supplementary Table S1). In line with the mouse data, we observed that - using FDR $<0.05$ and two-fold change cut-off in both cases - more transcripts displayed $\mathrm{m} 6 \mathrm{~A}$ methylation changes $(n=1246)$ than genes that were differentially expressed $(n=228)$ (Figure 4A; online supplementary Figure S6A and Table S4). The differentially expressed genes were mainly linked to processes of

Figure 2 m6A RNA methylation changes associated with heart hypertrophy and heart failure. (A) Echocardiographic phenotyping 1 week after transverse aortic constriction (TAC) (anterior wall thickness: sham $0.94 \mathrm{~mm}$, TAC $1.31 \mathrm{~mm}, P=0.0001$; ejection fraction: sham 61\%, TAC $59 \%, P=0.6172$ ). (B) Echocardiographic phenotyping 8 week after TAC (anterior wall thickness: sham $0.983 \mathrm{~mm}$, TAC $1.351 \mathrm{~mm}, P=0.0001$; ejection fraction: sham $59 \%$, TAC $46 \%, P=0.0002)$. Values represent mean \pm standard error of the mean $(n=6$ animals per group except for sham 8 weeks with 5 mice available). (C) Left panel: Bar chart showing the number of genes differentially methylated and differentially expressed in the TAC model for cardiac hypertrophy. Right panel: Pie charts showing the distribution of m6A RNA methylation changes across hyperand hypomethylated transcripts. $(D)$ Left panel: Bar chart showing the number of differentially m6A methylated and differentially expressed genes in the TAC model for heart failure. Right panel: Pie charts showing the distribution of m6A RNA methylation changes across hyperand hypomethylated transcripts. $(E)$ Distribution of hyper- and hypomethylated peaks along the gene body in the TAC model for cardiac hypertrophy. $(F)$ Gene ontology analysis (left panel) and integrated genome browser views of representative transcripts (right panel) hypo- and hypermethylated in cardiac hypertrophy. $(G)$ Distribution of hyper- and hypomethylated peaks along the gene body in heart failure. Significant enrichment of hypomethylation at 5'UTR was observed [hypomethylation: area under the curve (AUC) $=0.2733$, 95\% confidence interval (Cl) $0.2731-0.2735$; hypermethylation: $A \cup C=0.1617,95 \%(C l 0.1614-0.1619]$. $(H)$ Gene ontology analysis (left panel) and integrated genome browser views of representative transcripts (right panel) hypo- and hypermethylated in the TAC model for heart failure. 
A

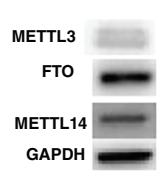

E

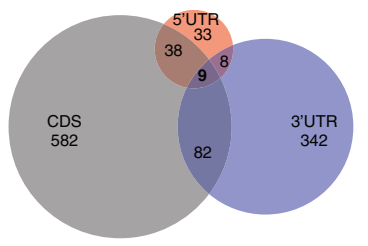

B

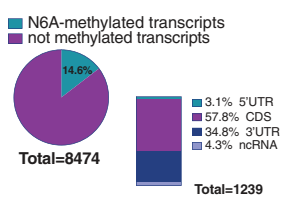

C

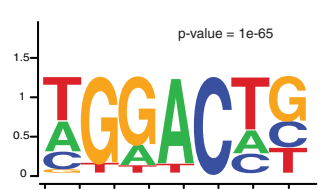

$\mathbf{F}$

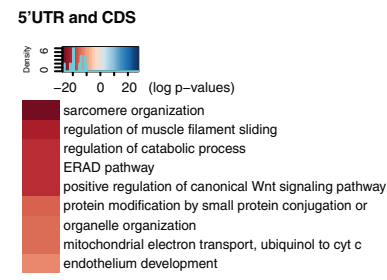

D

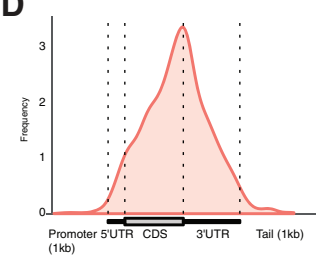

G

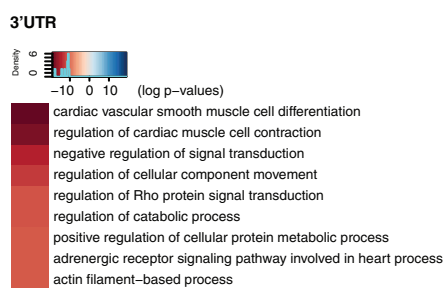

H

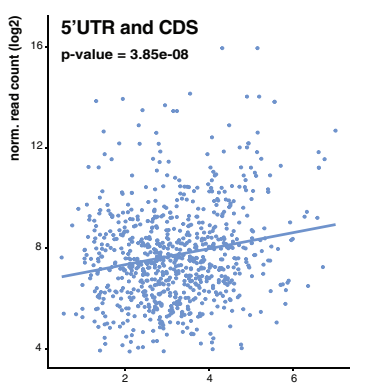

3'UTR p-value $=0.3248$

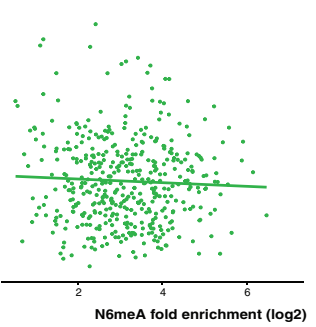

I

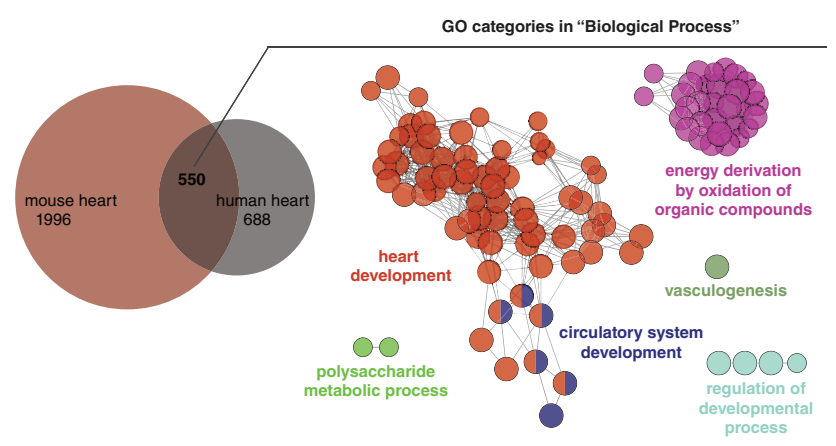

Figure 3 m6A RNA methylation in the healthy human heart. (A) Representative western blot images showing expression of FTO, METTL3 and METTLI4. (B) Pie and bar charts showing the amount and distribution of m6A RNA methylation in the human heart. (C) Sequence motif identified within m6A peaks. (D) Distribution of m6A peaks across mRNA transcripts. $(E)$ Venn diagram showing m6A marks across transcript regions. $(F)$ Heat map showing pathway analysis of transcripts that carry m6A methylation marks in $5^{\prime} U T R$ and $C D S$. (G) Heat map showing pathway analysis of transcripts that carry m6A methylation marks in $3^{\prime} U T R$. $(H)$ Correlation analysis between transcript level and m6A methylation at 5'UTR and CDS (left panel) and 3'UTR (right panel). (I) Left panel: Venn diagram depicting a significant overlap (representation factor 7.6, $P<2.37$ e-21) of genes encoding methylated transcripts in cardiac tissue of mice and humans. Right panel: Gene ontology categories for transcripts commonly observed in the mouse and human heart.

structural plasticity such as 'regulation of smooth cell proliferation', 'extracellular matrix organization' as well as 'metabolic function' (online supplementary Figure S6A). Similar to our findings in mice after 8 weeks of TAC (online supplementary Figure S3B), in human failing heart tissue, genes differentially expressed were substantially different from the transcripts that underwent differential m6A methylation (online supplementary Figure S6B and Table S5) providing further evidence that changes in $\mathrm{m} 6 \mathrm{~A}$ methylation and transcript level represent different cellular responses to cardiac stress.

We found that hypermethylated transcripts were mainly linked to processes that control the 'response to muscle stretch', 'response to growth factor' as well as 'heart morphogenesis' and metabolic processes (Figure 4B). Hypomethylated transcripts were associated with 'adrenergic receptor signalling in the heart', 'negative regulation of signal transduction', mitochondrial function and metabolic processes (Figure 4C). Comparison of differentially m6A methylated transcripts detected in mouse and human heart failure showed a significant overlap (403 transcripts, representation factor 7.8, $P<3.851 \mathrm{e}-251$ ) (Figure $4 D$ ). These transcripts code for proteins that are, for example, linked to 'cardiac muscle differentiation' and metabolic processes (Figure 4D). Future research is needed to analyse more heart biopsy material from patients. It will be interesting to see if the degree of m6A RNA methylation changes might be correlated to the severity of clinical parameters.

\section{Differential m6A methylation during heart failure is linked to transcripts with altered polysome binding}

The data obtained from the mouse model of heart failure and failed human heart samples suggest that changes in m6A mRNA 
A

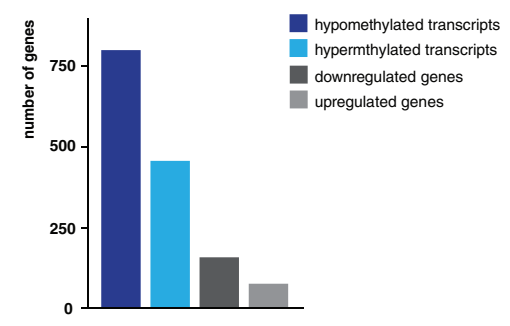

B

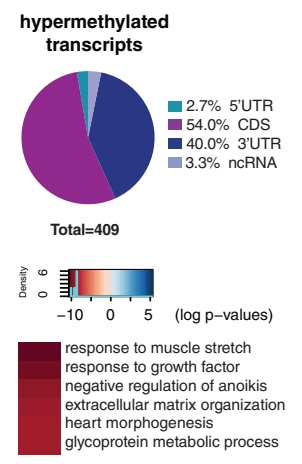

C

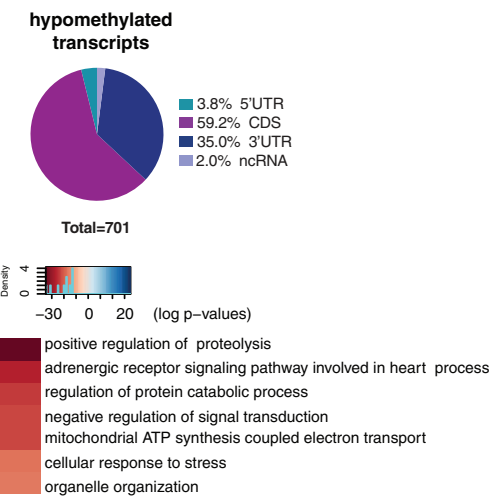

D

GO categories in "Biological Process"

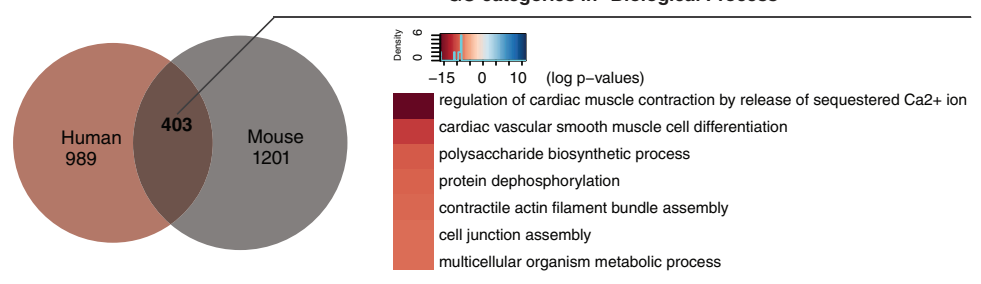

Figure 4 m6A RNA methylome in human end-stage heart failure. (A) Bar chart showing the number of transcripts differentially methylated and differentially expressed when comparing cardiac tissue from healthy individuals to patients suffering from end-stage heart failure ( $n=6 / g r o u p)$. (B) Upper panel: Pie chart showing the distribution of m6A RNA methylation changes across hypermethylated transcripts. Lower panel: Gene ontology terms identified for hypermethylated transcripts in human heart failure. (C) Upper panel: Pie chart showing the distribution of m6A RNA methylation changes across hypomethylated transcripts. Lower panel: Gene ontology terms identified for hypomethylated transcripts in human heart failure. $(D)$ Venn diagram showing the comparison of differentially methylated transcripts detected in end-stage human heart failure to differentially methylated transcripts identified in the heart failure mouse model. The heat map shows the pathways represented by commonly deregulated transcripts.

methylation might play a role in cardiac function. Generally, RNA methylation has been associated with an altered mRNA decay rate, ${ }^{20,21}$ suggesting that changes in m6A RNA methylation may affect transcript levels. However, altered transcript levels are unlikely to be the major cellular consequence of altered m6A RNA methylation in the failing heart given that the vast majority of transcripts that exhibited changes in $\mathrm{m} 6 \mathrm{~A}$ levels were not differentially expressed (online supplementary Figures S3A,B and S4). In line with these data, the correlation of differentially methylated transcripts with their expression levels in mice and humans was not different to genes at baseline expression (online supplementary Figure S3C). Previous studies have also suggested that RNA methylation could impact mRNA translation by affecting ribosome occupancy. ${ }^{22,23}$ To analyse this further, we performed polysome profiling to compare transcripts bound to translating ribosomes ${ }^{24}$ in mouse heart tissue 8 weeks post-sham/TAC.

We identified 225 transcripts that were significantly enriched or depleted from polysome fractions 8 weeks after TAC (Figure 5A and online supplementary Table S4). Transcripts depleted from polysomes were almost exclusively linked to metabolic processes, while those that were enriched mRNAs were involved in pathways such as calcium signalling, smooth muscle-related processes and apoptosis (Figure 5A). Differentially methylated and differentially polysome-bound transcripts 8 weeks after TAC showed a significant positive correlation of $(r=0.37, P<2.2 \mathrm{e}-16$; Figure $5 B)$, indicating an effect of $\mathrm{m} 6 \mathrm{~A}$ methylation on polysome binding. Interestingly, this correlation was specific to transcripts that underwent differential m6A methylation after TAC, since no such correlation was observed in the sham control group (online supplementary Figure S4).

In order to provide first evidence that altered polysome binding could be a mechanism by which m6A RNA methylation impacts on cardiac plasticity in the human heart as well, we compared the 403 transcripts that exhibited altered m6A methylation in both mouse and human heart failure (Figure 4D) with our mouse polysome-bound transcripts and found a significant positive correlation (Figure 5C). Enrichment analyses of these transcripts showed pathways essential for cardiac function and metabolic processes (Figure 5D). Furthermore, we verified differential m6A RNA methylation by qRT-PCR analysis for Calm 1 and some other mRNAs by analysing input and m6A immunoprecipitated RNA samples from heart tissue of mice after 8 weeks of TAC surgery with qRT-PCR (Figure 5E and online supplementary Figure S8). mRNAs of the selected candidate genes showed no change in RNA expression while their methylation levels differed in the failing heart. Western 
A

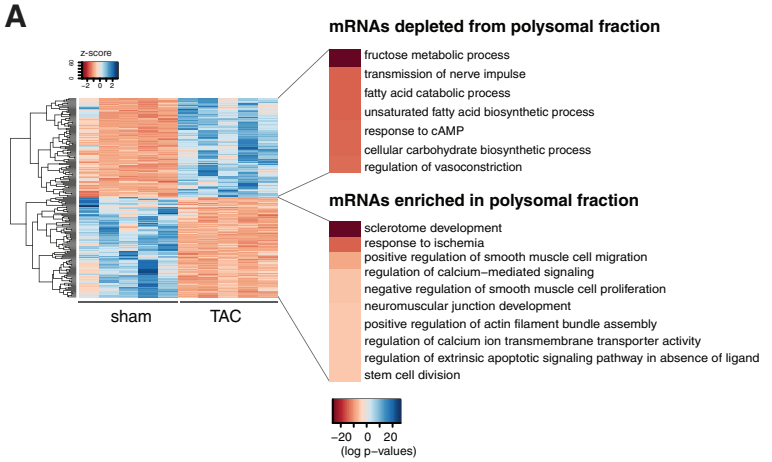

B

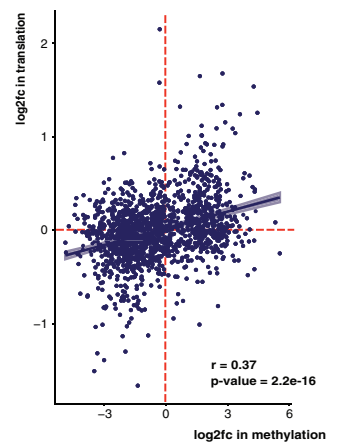

C

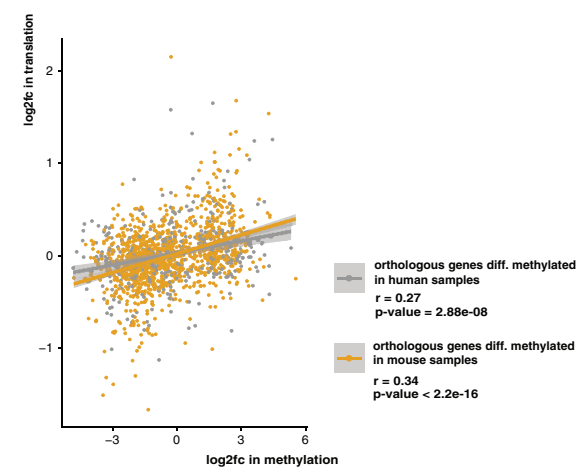

E

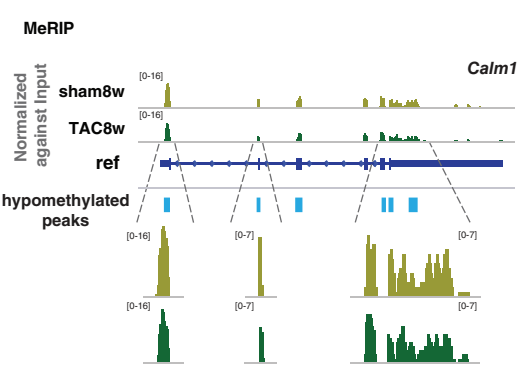

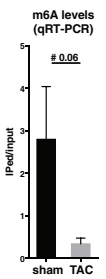

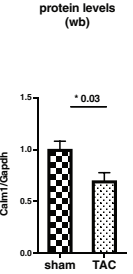

D

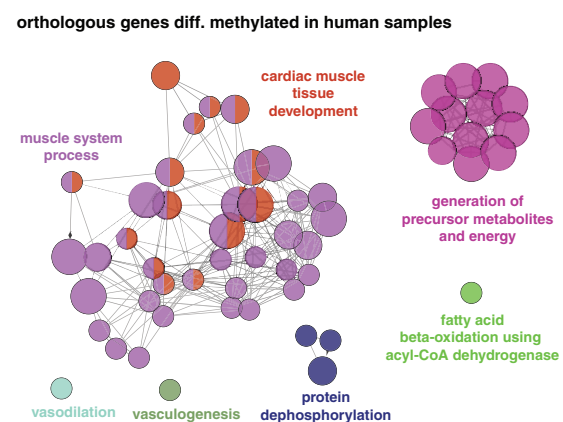

F
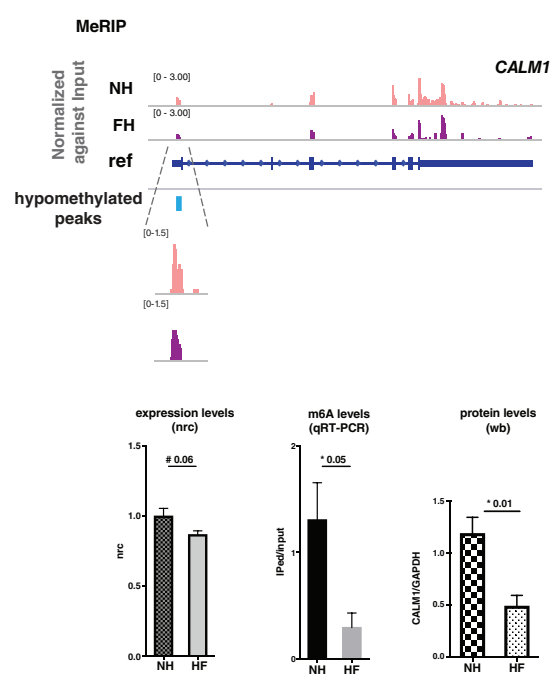

Figure 5 Changes in m6A RNA methylation during heart failure correlate with changes in polysome occupancy. (A) Heat map showing transcripts with significantly altered polysome association in the heart failure mouse model ( $n=5$ per group, Padj $<0.05$, cut-off of fold change was set at $>2.0$ for enriched mRNAs and $<0.5$ for depleted mRNAs) and the corresponding gene ontology categories (B). Comparison of transcripts exhibiting altered $\mathrm{m} 6 \mathrm{~A}$ methylation in the heart failure mouse model with polysome occupancy reveals a significant correlation $(r=0.37, P=2.2 e-16)$. (C) Human orthologous genes show significant correlation when calculated separately $(r=0.27, P=2.88 \mathrm{e}-08)$. ( $(D)$ Gene ontology categories for genes showing differential methylation in human failing heart tissue and differential binding to polysomes in the mouse model of heart failure. (E) Verification of methylation changes for Calm 1 transcript in the mouse model for failed heart [8 weeks after transverse aortic constriction (TAC)]. MeRIP analysis showed hypomethylation, which was confirmed by qRT-PCR analysis of m6A precipitated RNA ( $n=4$ per group, mean \pm standard errors are shown). Transcript levels of murine Calm 1 is not changed, while western blot analysis revealed significantly reduced amount of Calm 1 protein. $(F)$ Hypomethylation of CALM1 transcript identified by MeRIP was confirmed with qRT-PCR approach in failed human heart tissue. While transcript levels showed a trend toward downregulation in impaired heart, corresponding protein levels decrease significantly ( $n=3$ per group, mean \pm standard errors are shown). 
A

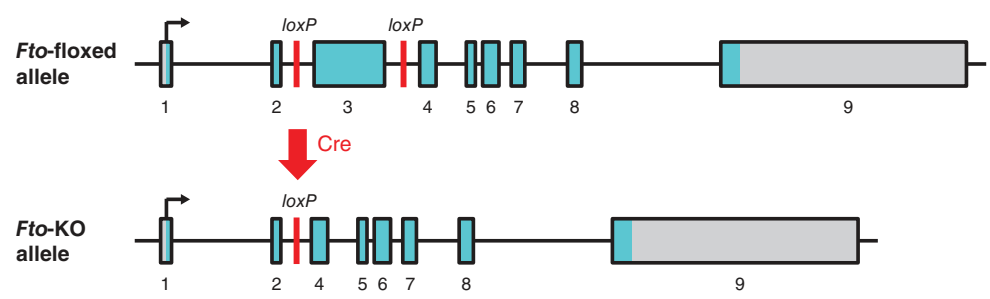

B

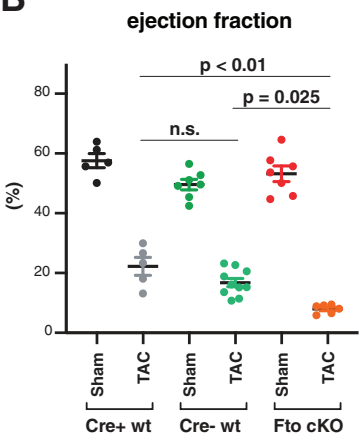

C

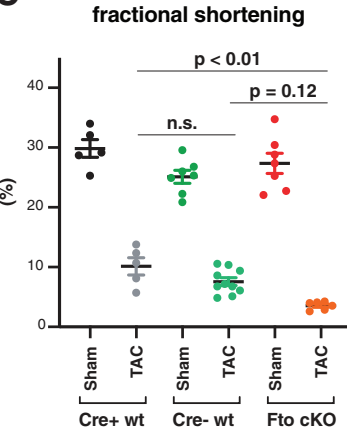

D
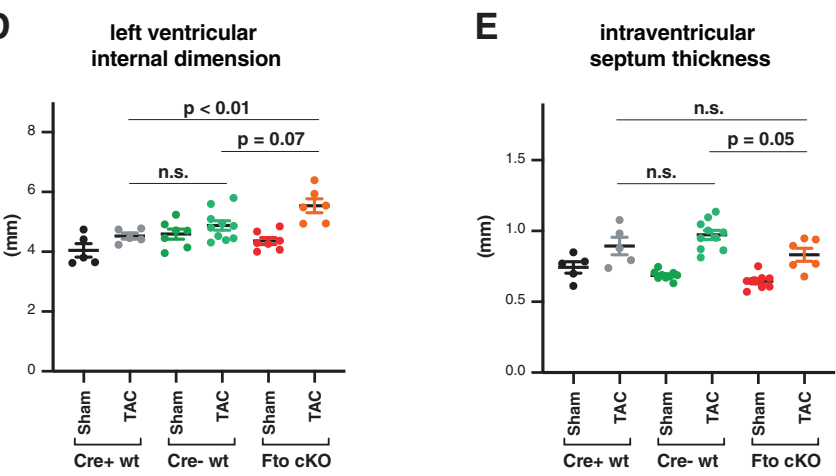

Figure 6 Fto-knockout mice show a worsened cardiac phenotype after transverse aortic constriction (TAC) surgery. (A) Scheme of generation Fto KO mice. Introns are 10x reduced while exon 2 and exon 3 are slightly enlarged for better visualization. Mice without Fto in cardiomyocytes showed significantly reduced ejection fraction $(B)$ and fractional shortening $(C)$, while degree of dilatation increases $(D, E)$. $n=5$ for Cre+ wild-type animals, $n=7$ for Cre- wild-type and Fto-KO mice. Mean values with standard errors are depicted.

blot analysis of Calm1 and Smyd1 proteins revealed decrease levels as well (Figure 5E and online supplementary Figure S8). The same results gave analysis of human heart samples - mRNA levels of CALM1 was not affected in failed heart tissue while hypomethylation revealed by MeRIP was confirmed with qRT-PCR and the protein level decreased significantly (Figure 5F). In summary, these data suggest that altered m6A methylation of mRNA affects polysome binding of the corresponding transcripts in the heart and thus has an impact on proteostasis in a transcription-independent manner.

\section{Fto-knockout mice show a worsened cardiac phenotype}

To provide further evidence that $\mathrm{m} 6 \mathrm{~A}$ levels play a role in normal cardiac function, we generated mice that lack m6A demethylase Fto in cardiomyocytes (Fto-KO) (Figure 6A and online supplementary Figure S10). Compared to Cre- wild-type (wt) and Cre+ wt control mice, Fto-cKO mice showed a more severe reduction in ejection fraction $(16.2 \%$ and $22.25 \%$ vs. $8.01 \%$, Cre- wt and Cre+ wt vs. Fto-cKO, respectively) and a higher degree of dilatation upon TAC surgery $(4.85$ and $4.52 \mathrm{~mm}$ vs. $5.54 \mathrm{~mm}$, Cre- wt and Cre+ wt vs. Fto-cKO, respectively) (Figure $6 B-E$ ). Thus, cardiac function depends on the presence of the Fto demethylase and the cellular m6A landscape.

\section{Discussion}

Our study reveals that (i) m6A RNA methylation is altered in heart hypertrophy and heart failure; (ii) m6A RNA methylation degree positively correlates with ribosomal occupancy, leading to 
increased and decreased protein levels for hyper- and hypomethylated transcripts, respectively; (iii) m6A RNA methylation levels affect protein abundance of genes that do not change their mRNA levels, pointing to the transcription-independent mechanism of translation regulation; (iv) modulation of the m6A RNA system by cardiomyocyte specific knockout of the demethylase Fto leads to a faster progression of heart failure with significant reduction in ejection fraction and increased dilatation.

\section{Changes in transcriptome and epitranscriptome in heart failure}

When we investigated m6A RNA methylation during heart failure development, we found that the number of RNAs with altered methylation levels was much higher than the number of genes that change their mRNA levels, meaning that changes in m6A RNA methylation exceed changes in gene expression. It is important to note that we applied the same cut-off to determine significant changes in either gene expression or m6A RNA methylation, thereby allowing a direct comparison. Interestingly, changes in $\mathrm{m} 6 \mathrm{~A}$ RNA methylation mainly occur in transcripts coding for proteins involved in cardiac signalling and metabolic processes whereas changes in gene expression were mainly linked to structural targets. Therefore, m6A RNA methylation is likely to influence the very early steps of gene expression regulation since transcripts encoding several transcription factors (FOXO1, FOXO4, ELF2, EIF5a), epigenetic proteins (SMYD1, DICER1, RBM20) and corresponding regulators of signalling pathways up-stream of gene expression (for example ERK and MDM2) are differentially methylated. Small changes in the expression of these 'regulatory' targets might lead to more profound changes of their downstream targets and therefore m6A RNA methylation could have a larger impact on the cardiac phenotype.

\section{Transcription-independent effect of m6A RNA methylation}

As m6A RNA methylation is present and furthermore changes also in transcripts that do not change their mRNA levels, m6A RNA methylation could affect mRNA translation and hence the proteome independent of transcription. For example, we could show, that the protein abundance of calmodulin 1 (Calm1) - a member of the important CaMKII signalling pathway - is reduced while its mRNA level is unaffected but m6A RNA methylation decreases. This shows that until now, the focus on RNA expression regulation is insufficient to describe the changes in protein abundance and that additional mechanisms - like m6A RNA methylation - can contribute to changes in the levels of proteins and, in our case, to heart failure development. This could also help to explain the often observed discrepancy between disease-associated changes in mRNA levels and the corresponding protein level. ${ }^{25,26}$

\section{Biological importance of m6A RNA methylation}

We reasoned that m6A marks on different regions of transcripts might have diverse influence on mRNA, like RNA stability, transport, ribosomal binding and decay. To our knowledge this is one of the first studies showing a link between transcript levels and the extent of m6A methylation at 5'UTR and CDS but not 3'UTR and non-coding RNAs. m6A RNA methylation was described to play a role in promoting mRNA decay. ${ }^{21}$ The correlation of $\mathrm{m} 6 \mathrm{~A}$ RNA methylation and transcript levels in cardiac tissue is however comparatively small, suggesting that m6A RNA methylation in the heart does not have a major effect on RNA decay. Indeed, studies in other tissues reported that m6A RNA methylation also affects processes such as translation initiation and/or efficiency. ${ }^{22,23}$ Since we found a correlation between m6A RNA methylation and ribosomal occupancy, we believe that this mechanism is present in the heart. Also m6A RNA methylation can affect other RNA-based processes, for example RNA transport. ${ }^{14,27,28}$ These different consequences of m6A RNA methylation are most likely mediated by specific m6A RNA methylation reader proteins ${ }^{10,20}$ but the details of such a regulation are currently not well understood. More research is needed to increase the understanding of the functions of $\mathrm{m} 6 \mathrm{~A}$ methylation in the heart and the mechanisms by which these effects are exerted.

\section{Mechanisms underlying m6A RNA methylation changes}

Our study, showing differential m6A methylation in cardiac tissue in case of heart failure, is in agreement with recent reports. ${ }^{15,29,30}$ Mathiyalagan et al. ${ }^{15}$ showed that $\mathrm{m} 6 \mathrm{~A}$ methylation is involved in the regeneration of the infarct/peri-infarction area, whereas Dorn et al. ${ }^{29}$ and Kmietczyk et al. ${ }^{30}$ reported that the hypertrophic response is altered in Mettl3-knockout mice leading to heart failure after TAC. This is interesting since our study shows that also Fto-cKO leads to a faster progression of heart failure with reduced hypertrophy. Therefore, deletion of the RNA methyltransferase (Mettl3) as well as the RNA demethylase (Fto) may impair the response to pressure overload. These data are in line with our observation that hyper- and hypomethylated transcripts are detected in response to heart insufficiency and suggest that the regulatory function of $\mathrm{m} 6 \mathrm{~A}$ RNA methylation is complex. We speculate that disturbance in either direction is linked to compromised cardiac function. It will be interesting to study to what extent other factors, including genetic predisposition, influence the direction and degree of m6A RNA methylation changes in cardiac diseases, thereby offering the possibility for stratified therapies. Moreover, since it has been shown that $\mathrm{m6 \textrm {A }}$ methylation can have a stimulatory ${ }^{23}$ or an inhibitory effect ${ }^{31}$ on translation, probably the location of the methylation mark within a transcript might determine the functional consequences. Further research is needed to study these possibilities.

One approach would be to elucidate the mechanisms that lead to altered m6A RNA methylation in cardiac diseases. We did not observe any changes of mRNA or protein levels of the key regulators of m6A RNA methylation (online supplementary Figure S7). The only exemption was a decrease of METTL3 protein in human tissue from heart failure patients, suggesting that the manipulation of Mettl3 and its counterplay Fto in a cell-specific and temporally-controlled manner in mice and in human-induced 
pluripotent stem cell-derived cardiomyocytes might be a suitable approach for further studies. However, these regulators of m6A RNA methylation are also regulated via post-translational modifications $^{13,32}$ and in case of FTO, shuttling between nucleus and cytoplasm has been reported. ${ }^{33}$ Whether any of these processes could explain the changes in m6A RNA methylation alteration during heart failure development remains to be investigated.

In conclusion, our data shows that m6A RNA methylation is deregulated during heart failure progression. m6A RNA methylation changes are linked to changes in protein translation, even for genes that do not change their mRNA levels. This uncovers a new mechanism of translation regulation, independent of transcription. Therefore, our data suggest that modulation of epitranscriptomic processes, such as m6A RNA methylation, might be an interesting target for therapeutic interventions.

\section{Clinical perspective}

Here we could show that methylation of RNA is changed in cardiac hypertrophy and heart failure. We suggest a novel mechanism where RNA methylation influences RNA-ribosome interaction and leads to a change in protein expression and heart failure progression. Importantly, this is also true for targets that show no change in RNA expression level. This shows that protein expression regulation in heart failure occurs partially only on the translational level and without changes in DNA to RNA transcription. Therefore, this novel mechanism opens potential new treatment options for heart failure.

\section{Supplementary Information}

Additional supporting information may be found online in the Supporting Information section at the end of the article.

Methods S1. Supplementary methods.

Figure S1. m6A landscape of non-coding RNA. (A) $1.15 \%$ of all detected $\mathrm{m} 6 \mathrm{~A}$ peaks in normal heart tissue of mouse $(n=3208)$ were mapped to non-coding RNA $(n=37)$. (B) Correlation analysis between the methylation levels of non-coding RNA and transcript abundance of the same molecules. In contrast to the finding for $5^{\prime}$ UTR and coding sequence, no relationship was revealed for non-coding RNAs between these two values ( $r=0.06, P=0.7188)$.

Figure S2. Gene expression changes in hypertrophic and failing heart of mouse. (A) RNA-Seq followed by differential gene expression revealed 73 genes upregulated and 83 genes downregulated after 1 week of TAC surgery ( $\log 2 \mathrm{FC}>1, \operatorname{Padj}<0.05)$. Lower part shows GO categories identified for deregulated genes. (B) Eight weeks after TAC surgery, 144 genes showed increased levels of transcripts while 91 genes were detected with reduced transcript abundance. Pathway analysis of differentially expressed genes showed that both metabolic and cardiac function are affected. (C) Venn diagram showing that 71 genes were differentially expresses
1 week and 8 weeks after TAC surgery. Gene enrichment analysis indicated that those genes participate in important cardiac pathways.

Figure S3. Differentially methylated transcripts outnumber differentially expressed RNAs. (A) One week after TAC surgery 217 genes showed deregulation at the transcript levels while RNAs generated from 1611 loci were detected as differentially methylated. Only 78 genes encoded transcripts differentially expressed and differentially methylated at the same time (Log2FC > 1, Padj < 0.05 cut-offs were used for both, differential expression and differential methylation analysis). (B) Left panel: 47 genes showed differential methylation and differential expression 8 weeks after surgery compared to their control group. Many more genes with differentially methylated RNAs $(n=1182)$ than differentially expressed $(n=174)$ were detected. Right panel: Venn diagram of differentially methylated, differentially transcribed and differentially translated genes in the heart failure model. (C) Correlation analysis between the levels of methylation and abundance of transcripts that changed their methylation degrees in failed heart tissue in mouse (left panel) and human (right panel). Figure S4. Analysis of correlation between the levels of methylation and polysome occupancy in healthy heart tissue of mouse. No correlation $(r=0.003, P=0.8765)$ was revealed between the levels of RNA N6A methylation and polysome binding for those RNAs in control group of mouse.

Figure S5. Epitranscriptome of human heart tissue. (A) Distribution of $\mathrm{m} 6 \mathrm{~A}$ peaks across IncRNA. (B) No correlation was found between the levels of methylation and abundance for non-coding RNAs ( $r=-0.09, P=0.6895)$. (C) Guitar plots showing distribution of peaks with gained and lost methylation levels in human patients with heart failure.

Figure S6. Changes in transcriptome of heart tissue in human patients. (A) Differential gene expression analysis revealed 228 genes with altered transcript levels (Log2FC $>1$, Padj <0.05). mRNAs from only 30 loci were differentially methylated at the same time (B). As shown in the mouse model of heart failure, in human patients in compromised heart tissue much more transcripts show differential methylation $(n=1249)$ than differential expression $(n=228)$. Pathway analysis of differentially expressed genes revealed importance of those proteins in organization of actomyosin structure, response to stress and formation of extracellular matrix.

Figure S7. Expression of members of m6A machinery. $(A)$ RNA-Seq data did not reveal differential expression of m6A writers, readers and erasers in mouse model of heart hypertrophy $(A)$ and heart failure $(B)$. No changes were detected on the level of proteins for Fto, Mettl14 and Mettl16 genes while Mettl3 showed mild reduction of protein levels in mouse failing heart samples. (C) RNA expression analysis of different genes related to m6A in human samples lead to no drastic changes in transcript levels in human failing heart samples. Western blot analysis of METTL3, METTL14 and FTO protein showed no difference in abundance between healthy and failed human heart tissue.

Figure S8. Verification of differential m6A methylation by qRT-PCR. Smyd1, Gata6 and Rnd3 transcripts were selected 
to verify MeRIP data by $q R T-P C R$ of $m 6 A$ immunoprecipitated samples from heart RNA obtained from the new batch of animals 8 weeks after TAC surgery( $n=4$ per group). mRNAs from Smyd 1 and Gata6 showed hypomethylation, while for Rnd3 mRNAs hypermethylation was confirmed by showing increased trend of ratio of IPed/input in TAC mice compared to sham animals. Western blot analysis of Smyd1 protein revealed reduction.

Figure S9. Western blot analysis of m6A RNA machinery. Full western blots are provided for main members of RNA methyltransferase and RNA demethylase for both mouse and human heart tissues.

Figure S10. Generation of Fto-cKO mice. A machinery. $(A)$ qRT-PCR analysis shows reduction of Fto mRNA by $80 \%$ in heart tissue of Fto-cKO mice. $(B)$ Western blot analysis revealed depletion of Fto protein from isolated cardiomyocytes of Fto-cKO animals, whereas in protein extract from the whole left ventricle contains some detectable Fto protein.

Table S1. Patient characteristics and received treatments.

Table S2. Parameters of healthy human donors.

Table S3. m6A RNA methylation in mouse heart hypertrophy. Lists of differentially methylated and differentially expressed genes are provided

Table S4. m6A RNA methylation in mouse heart failure. Lists of differentially methylated and differentially expressed genes are provided. Transcripts showing differential polysome binding are listed

Table S5. m6A RNA methylation in huma heart failure. Lists of differentially methylated and differentially expressed genes are provided

\section{Acknowledgements}

We gratefully acknowledge the excellent technical assistance of S. Koszewa, A. Kretschmar, S. Zaffar, B. Knocke, R. Blume, and M. Zoremba. Great thanks to Chantal and Ferdinand for making this project possible. AF, GH were supported by the DFG under Germany's Excellence Strategy - EXC 2067/1 390729940.

\section{Funding}

This work was supported by the German Research Foundation (DFG, SFB1002 project D04 to A.F. \& K.T., D01 to G.H. and BO3442/2-2 in SPP1784 to M.T.B). The work was furthermore supported by the following funds to A.F.: DFG project FI179, the DFG priority program 1738 (FI981), an ERC consolidator grant DEPICODE (648898), the BMBF projects ENERGI (01GQ1421A) and Intergrament (01ZX1314D), and funds from the German Center for Neurodegenerative Diseases (DZNE) and German Center for cardiovascular research (DZHK).

Conflict of interest: none declared.

\section{References}

1. Savarese G, Lund LH. Global public health burden of heart failure. Card Fail Rev 2017;3:7-11.

2. Azevedo PS, Polegato BF, Minicucci MF, Paiva SAR, Zornoff LAM. Cardiac remodeling: concepts, clinical impact, pathophysiological mechanisms and pharmacologic treatment. Arq Bras Cardiol 2016;106:62-69.
3. Toischer K, Rokita AG, Unsöld B, Zhu W, Kararigas G, Sossalla S, Reuter SP, Becker A, Teucher N, Seidler T, Grebe C, Preuss L, Gupta SN, Schmidt K, Lehnart SE, Krüger M, Linke WA, Backs J, Regitz-Zagrosek V, Schäfer K, Field LJ, Maier LS, Hasenfuss G. Differential cardiac remodeling in preload versus afterload. Circulation 2010;122:993-1003.

4. Chatterjee K. Pathophysiology of systolic and diastolic heart failure. Med Clin North Am 2012;96:891-899.

5. Anand P, Brown JD, Lin CY, Qi J, Zhang R, Artero PC, Alaiti MA, Bullard J, Alazem K, Margulies KB, Cappola TP, Lemieux M, Plutzky J, Bradner JE, Haldar SM. BET bromodomains mediate transcriptional pause release in heart failure. Cell 2013;154:569-582.

6. Steenman M, Chen Y-W, Le Cunff M, Lamirault G, Varró A, Hoffman E, Léger Jj. Transcriptomal analysis of failing and nonfailing human hearts. Physiol Genomics 2003;12:97-112.

7. Gilsbach R, Schwaderer M, Preissl S, Grüning BA, Kranzhöfer D, Schneider $P$, Nührenberg TG, Mulero-Navarro S, Weichenhan D, Braun C, Dreßen M, Jacobs AR, Lahm H, Doenst T, Backofen R, Krane M, Gelb BD, Hein L. Distinct epigenetic programs regulate cardiac myocyte development and disease in the human heart in vivo. Nat Commun 2018;9:391.

8. Fu $Y$, Dominissini D, Rechavi G, He C. Gene expression regulation mediated through reversible m6A RNA methylation. Nat Rev Genet 2014;15:293-306.

9. Roundtree IA, Evans ME, Pan T, He C. Dynamic RNA modifications in gene expression regulation. Cell 2017;169:1187-1200.

10. Yang Y, Hsu PJ, Chen YS, Yang YG. Dynamic transcriptomic m6A decoration: writers, erasers, readers and functions in RNA metabolism. Cell Res 2018;28:616-624.

11. Warda AS, Kretschmer J, Hackert $P$, Lenz C, Urlaub H, Höbartner C, Sloan $\mathrm{KE}$, Bohnsack MT. Human METTL16 is a N6-methyladenosine (m6A) methyltransferase that targets pre-mRNAs and various non-coding RNAs. EMBO Rep 2017;18:2004-2014.

12. Yue $\mathrm{Y}$, Liu J, He C. RNA N6-methyladenosine methylation in post-transcriptional gene expression regulation. Genes Dev 2015;29:1343-1355.

13. Yeo GS, O'Rahilly S. Uncovering the biology of FTO. Mol Metab 2012;1:32-36.

14. Zheng G, Dahl JA, Niu Y, Fedorcsak P, Huang CM, Li CJ, Vågbø CB, Shi Y, Wang WL, Song SH, Lu Z, Bosmans RP, Dai Q, Hao YJ, Yang X, Zhao WM, Tong WM, Wang XJ, Bogdan F, Furu K, Fu Y, Jia G, Zhao X, Liu J, Krokan HE, Klungland A, Yang YG, He C. ALKBH5 is a mammalian RNA demethylase that impacts RNA metabolism and mouse fertility. Mol Cell 2013;49:18-29.

15. Mathiyalagan P, Adamiak M, Mayourian J, Sassi Y, Liang Y, Agarwal N, Jha D, Zhang S, Kohlbrenner E, Chepurko E, Chen J, Trivieri MG, Singh R, Bouchareb R, Fish K, Ishikawa K, Lebeche D, Hajjar RJ, Sahoo S. FTO-dependent N6-methyladenosine regulates cardiac function during remodeling and repair. Circulation 2019;139:518-532.

16. Cui $X$, Meng J, Zhang S, Chen $Y$, Huang $Y$. A novel algorithm for calling mRNA m6A peaks by modeling biological variances in MeRIP-seq data. Bioinformatics 2016;32:i378-i385.

17. Dominissini D, Moshitch-Moshkovitz S, Schwartz S, Salmon-Divon M, Ungar L, Osenberg S, Cesarkas K, Jacob-Hirsch J, Amariglio N, Kupiec M, Sorek R, Rechavi G. Topology of the human and mouse m6A RNA methylomes revealed by m6A-seq. Nature 2012;485:201-206.

18. Chang M, Lv H, Zhang W, Ma C, He X, Zhao S, Zhang ZW, Zeng YX, Song S, Niu $Y$, Tong WM. Region-specific RNA m6A methylation represents a new layer of control in the gene regulatory network in the mouse brain. Open Biol 2017;7:170166.

19. Meyer KD, Saletore Y, Zumbo P, Elemento O, Mason CE, Jaffrey SR. Comprehensive analysis of mRNA methylation reveals enrichment in $3^{\prime}$ UTRs and near stop codons. Cell 2012;149:1635-1646.

20. Shi H, Wang X, Lu Z, Zhao BS, Ma H, Hsu PJ, Liu C, He C. YTHDF3 facilitates translation and decay of N6-methyladenosine-modified RNA. Cell Res 2017;27:315-328.

21. Wang X, Lu Z, Gomez A, Hon GC, Yue Y, Han D, Fu Y, Parisien M, Dai Q, Jia G, Ren B, Pan T, He C. N6-methyladenosine-dependent regulation of messenger RNA stability. Nature 2014;505:117-120.

22. Bodi Z, Bottley A, Archer N, May ST, Fray RG. Yeast m6A methylated mRNAs are enriched on translating ribosomes during meiosis, and under rapamycin treatment. PLoS One 2015;10:e0132090.

23. Meyer KD, Patil DP, Zhou J, Zinoviev A, Skabkin MA, Elemento O, Pestova TV, Qian SB, Jaffrey SR. 5'UTR m6A promotes cap-independent translation. Cell 2015;163:999-1010.

24. Chassé H, Boulben S, Costache V, Cormier P, Morales J. Analysis of translation using polysome profiling. Nucleic Acids Res 2017;45:e15.

25. Cenik C, Cenik ES, Byeon GW, Grubert F, Candille SI, Spacek D, Alsallakh B, Tilgner H, Araya CL, Tang H, Ricci E, Snyder MP. Integrative analysis of RNA, translation, and protein levels reveals distinct regulatory variation across humans. Genome Res 2015;25:1610-1621. 
26. de Sousa Abreu R, Penalva LO, Marcotte EM, Vogel C. Global signatures of protein and mRNA expression levels. Mol Biosyst 2009;5:1512-1526.

27. Yang $X$, Yang $Y$, Sun BF, Chen YS, Xu JW, Lai WY, Li A, Wang X, Bhattarai DP, Xiao W, Sun HY, Zhu Q, Ma HL, Adhikari S, Sun M, Hao YJ, Zhang B, Huang CM, Huang N, Jiang GB, Zhao YL, Wang HL, Sun YP, Yang YG. 5-Methylcytosine promotes mRNA export - NSUN2 as the methyltransferase and ALYREF as an $\mathrm{m}^{5} \mathrm{C}$ reader. Cell Res 2017;27:606-625.

28. Roundtree IA, Luo GZ, Zhang Z, Wang X, Zhou T, Cui Y, Sha J, Huang X, Guerrero L, Xie P, He E, Shen B, He C. YTHDC1 mediates nuclear export of N6-methyladenosine methylated mRNAs. Elife 2017;6:e31311.

29. Dorn LE, Lasman L, Chen J, Xu X, Hund TJ, Medvedovic M, Hanna JH, van Berlo $\mathrm{JH}$, Accornero F. The N6-methyladenosine mRNA methylase METTL3 controls cardiac homeostasis and hypertrophy. Circulation 2019;139:533-545.
30. Kmietczyk V, Riechert E, Kalinski L, Boileau E, Malovrh E, Malone B, Gorska A, Hofmann C, Varma E, Jürgensen L, Kamuf-Schenk V, Altmüller J, Tappu R, Busch M, Most P, Katus HA, Dieterich C, Völkers M. m6A-mRNA methylation regulates cardiac gene expression and cellular growth. Life Sci Alliance 2019;2:e201800233.

31. Slobodin B, Han R, Calderone V, Vrielink JA, Loayza-Puch F, Elkon R, Agami R. Transcription impacts the efficiency of mRNA translation via co-transcriptional N6-adenosine methylation. Cell 2017;169:326-337.e12.

32. Wang P, Doxtader KA, Nam Y. Structural basis for cooperative function of Mettl3 and Mettl14 methyltransferases. Mol Cell 2016;63:306-317.

33. Gulati P, Avezov E, Ma M, Antrobus R, Lehner P, O’Rahilly S, Yeo GS. Fat mass and obesity-related (FTO) shuttles between the nucleus and cytoplasm. Biosci Rep 2014;34:e00144. 